Optical Power Reduction for Multiple-Subcarrier Modulated Indoor Wireless Optical Channels 


\title{
OPTICAL POWER REDUCTION FOR MULTIPLE-SUBCARRIER MODULATED INDOOR WIRELESS OPTICAL CHANNELS
}

\author{
By \\ WEIWEI KANG, \\ B.Eng. (Information Engineering) \\ Beijing Institute of Technology, Beijing, China, 2004
}

\begin{abstract}
A Thesis
Submitted to the Department of Electrical \& Computer Engineering and the School of Graduate Studies in Partial Fulfilment of the Requirements for the Degree of Master of Applied Science
\end{abstract}

McMaster University

(C) Copyright by Weiwei Kang, July 2006 
Master of Applied Science (2006)

McMaster University

(Electrical \& Computer Engineering)

Hamilton, Ontario

TITLE:

Optical Power Reduction for Multiple-Subcarrier Modu-

lated Indoor Wireless Optical Channels

AUTHOR:

Weiwei Kang

B.Eng. (Information Engineering)

Beijing Institute of Technology, Beijing, China, 2004

SUPERVISOR: $\quad$ Dr. Steve Hranilovic

NUMBER OF PAGES: $\quad$ xv, 164 


\section{Dedications:}

To my parents Xiaolu Kang and Shumin Liu, my grandmother Lianying Huang, my uncle Shihuai Kang and aunt Anna Li, and Shuang Yang 


\section{Abstract}

Multiple-subcarrier modulated (MSM) indoor wireless optical systems provide highspeed bandwidth-efficient communication as well as multi-access capability at low cost. A major drawback of such systems is the high average optical power requirement. To address this problem, trellis codes are designed for data transmission realizing an average optical power reduction as high as $0.95 \mathrm{~dB}$. A simultaneous peak optical power reduction up to $0.44 \mathrm{~dB}$ is also possible by employing this technique.

Moreover, the unregulated bandwidth available in wireless optical channels is ex-

ploited to reduce the average optical power in MSM wireless optical systems. Data transmission is confined in a bandwidth located near DC, while reserved carrier signals are designed outside the data bandwidth to reduce the average optical power. Distorted out-of-band signals at higher frequency are removed at the receiver by low-pass filtering and not used for detection. To design the out-of-band carrier amplitudes, both a standard optimization algorithm over the set of real numbers and an exhaustive search over discrete constellation are proposed. It is shown that significant average optical power reduction as high as $2.56 \mathrm{~dB}$ is achieved.

It is assumed that a symbol-by-symbol bias is used in MSM wireless optical systems since it offers significant optical power reduction. The received DC bias level provides a degree of diversity and is used at the receiver to improve detection. It is also used to design trellis codes with better distance properties. In this manner, an additional average optical power reduction up to $0.50 \mathrm{~dB}$ is achieved with a simultaneous peak power reduction of $0.46 \mathrm{~dB}$ for MSM wireless optical systems. 


\section{Acknowledgments}

I would like to express my deep and sincere gratitude to my supervisor, Dr. Steve Hranilovic. The guidance and encouragement I received from him are crucial for the completion of this work. His passion for research inspired me to further pursue my own academic career in the future.

I would like to thank Dr. Timothy N. Davidson for his insightful comments and interests in my work. I would also like to thank Dr. Thiagalingam Kirubarajan for carefully reviewing my thesis. My gratitude also goes to my colleagues at McMaster University for their helpful conversations regarding this work and for their friendship.

My parents Xiaolu Kang and Shumin Liu, my grandmother Lianying Huang, my uncle Shihuai Kang and aunt Anna Li, and Shuang Yang have always been a source of love and support. This work reflects the constant care and encouragement I have received from them. 


\section{Contents}

1 Introduction 1

1.1 Context . . . . . . . . . . . . . . . . . . . 1

1.2 Current Wireless Optical System Implementations . . . . . . . . 6

1.2.1 Wireless Optical System Architectures . . . . . . . . . . 6

1.2.2 Channel Model, Baseband Modulation and Coding Schemes . . . . . . . . . . . . . . . . . 11

1.2.3 Experimental Links and Commercial Systems . . . . . . . 13

1.2.4 Multiple-Subcarrier Modulation (MSM) . . . . . . . . 14

1.3 Electrical MSM PAPR Reduction . . . . . . . . . . . . . . . . 16

1.4 Average Optical Power Reduction Techniques for MSM Wireless Optical Systems . . . . . . . . . . . . . . . . . 20

1.5 Research Direction . . . . . . . . . . . . . . . . . . . . 22

1.6 Thesis Structure . . . . . . . . . . . . . . . . . . . . . 24

2 Channel Model $\quad 25$

2.1 Basic Channel Structure . . . . . . . . . . . . . . . . . . 25

2.2 Noise . . . . . . . . . . . . . . . . . . . . . . . . 30

2.3 Amplitude constraints . . . . . . . . . . . . . . . . . 33

2.4 Channel Modeling . . . . . . . . . . . . . . . . . . . 35

2.5 Signal Space Concepts . . . . . . . . . . . . . . . . . 36 
2.6 MSM Wireless Optical Channels . . . . . . . . . . . . . . . . . . 39

2.7 Figures of Merit . . . . . . . . . . . . . . . 43

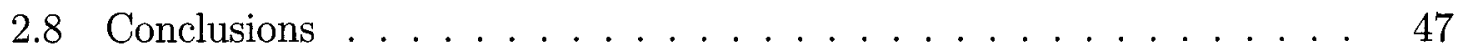

3 In-Band Trellis Coding and DC Detection 49

3.1 Trellis-coded Modulation . . . . . . . . . . . . . . . . . . 51

3.1.1 Baseline uncoded QPSK system . . . . . . . . . . . . . 51

3.1 .2 Trellis-coded System . . . . . . . . . . . . . . . . 53

3.2 Trellis-Coded MSM (TC-MSM) $\ldots \ldots \ldots \ldots \ldots$

3.2 .1 MSM constellation and expansion . . . . . . . . 58

3.2 .2 Code Design . . . . . . . . . . . . . . . . . . . . . . 60

3.3 Trellis-Coded Partitioned-MSM (TC-PMSM) . . . . . . . . . . . 65

3.4 Simulation Results . . . . . . . . . . . . . . . . . . 70

3.5 DC Level Detection . . . . . . . . . . . . . . . . . . . . 80

3.6 Conclusions . . . . . . . . . . . . . . . . . 85

4 Out-of-Band Carrier Design $\quad 87$

4.1 Problem Formulation . . . . . . . . . . . . . . . . . . . 88

4.2 Optimization of Amplitudes Over Real Numbers . . . . . . . . . . . 92

4.2.1 Formulation of the optimization problem . . . . . . . 93

4.2 .2 Performance Analysis . . . . . . . . . . . . . . . . . 95

4.2 .3 Bounding the estimation error . . . . . . . . . . . 101

4.2 .4 Conclusions . . . . . . . . . . . . . . . . . . . . . 108

4.3 Optimization of Amplitudes Over a Discrete Constellation . . . . . 108

4.3.1 Optimization by an Exhaustive Search . . . . . . . . 109

4.3 .2 Performance Analysis . . . . . . . . . . . . . . . . . . . . . 111

4.3 .3 Conclusions . . . . . . . . . . . . . . . . . . . . . . . . 119

4.4 Out-of-band techniques with reduced memory requirements . . . . . 119 
4.5 Out-of-band carriers applied to trellis-coded systems with DC detection technique . . . . . . . . . . . . . . . . . . 124

4.6 Conclusions . . . . . . . . . . . . . . . . . . . . . . . . 124

5 Discussions and Conclusions 132

5.1 Discussions on average optical power reduction techniques . . . . 135

5.2 Future directions . . . . . . . . . . . . . . . . . . . . . 139

A Performance of using $L=2$ and $L=3$ out-of-band carriers 141 


\section{List of Figures}

1.1 Wireless optical base stations connecting terminals inside a building and forming a network, $\mathrm{S}=$ base station, $\mathrm{T}=$ terminal $[1] \ldots \ldots$

1.2 Classification of wireless optical system architectures [2] . . . . . 7

1.3 Diffuse Wireless Optical Communication System [3] . . . . . . . 8

1.4 Illustration of a tracked wireless optical system [4] . . . . . . . 9

1.5 Illustration of a multi-spot diffusing configuration [5] . . . . . 10

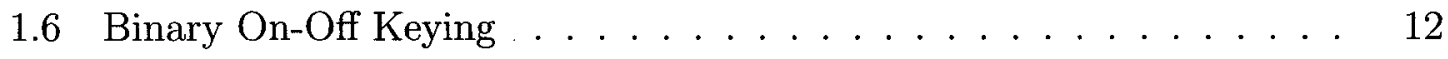

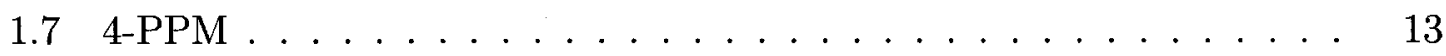

1.8 An example of multiple subcarrier modulation . . . . . . . . . . . 14

1.9 Illustration of the expanded constellation of the tone injection method 19

2.1 Intensity modulated, direct detection wireless optical channel [3] . . 26

2.2 (a) Top view of the measured room. $\mathrm{T}$ is the transmitter location. Receiver is located at $\mathrm{R}$ and additional measurements have been performed at locations r. (b) Measured channel magnitude response. [6] .

2.3 Optical power spectra of common background light sources. Spectra have been scaled to have the same maximum value. [2] . . . . . .

2.4 Detected electrical power spectrum of infrared emission from a fluorescent lamp driven by a $22 \mathrm{KHz}$ electronic ballast [7] . . . . . . . . . 32

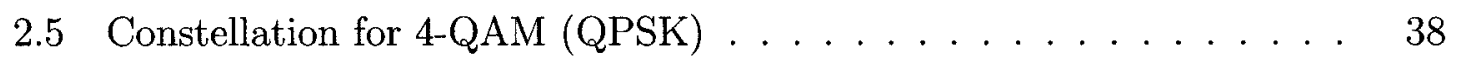

2.6 Conventional MSM wireless optical transmitter $\ldots \ldots \ldots$ 
2.7 Two-carrier MSM constellation with QPSK employed on each carrier and a symbol-by-symbol bias . . . . . . . . . . . . . . . . . 40

2.8 Conventional MSM wireless optical receiver . . . . . . . . 43

2.9 Time-domain rectangular pulse and its frequency-domain sinc pulse . 44

2.10 Dense packing and coarse packing of subcarriers . . . . . . . . . 45

2.11 Constellation and basis function for OOK $\ldots \ldots \ldots$. . . . 47

3.1 Unit-energy QPSK constellation . . . . . . . . . . . . . . . . 52

3.2 Set Partitioning of a 8-PSK constellation $[8] \ldots \ldots \ldots$

3.3 A 4-state convolutional encoder . . . . . . . . . . . . . . 55

3.4 Trellis diagram for the 4-state convolutional code in Figure $3.3 \ldots \ldots$

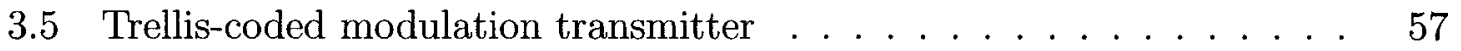

3.6 Trellis-coded MSM wireless optical transmitter . . . . . . . . . . 58

3.7 MSM constellation point consisting of 4 sub-constellations . . . . . 58

3.8 5-APSK and 9-APSK sub-constellations . . . . . . . . . . 59

3.9 TC-MSM encoder and mapping block . . . . . . . . . . . . . . 60

3.10 Trellis structure and MSM constellation for $K=2, M=2, W=2$

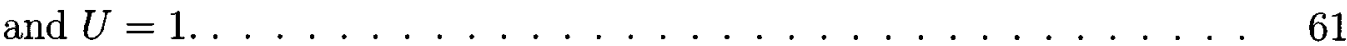

3.11 Partitioning of the 5-APSK sub-constellation. . . . . . . . . . 66

3.12 Trellis structure for $W=5, U=2, M=2$ and $K=5 \ldots \ldots 67$

3.13 MSM constellation points chosen from PA1 given inputs $b^{2}, b^{3}$ and $b^{4} \quad 68$

3.14 Normalized average optical power $(\rho)$ versus bandwidth efficiency $(\eta)$ for TC-MSM and TC-PMSM . . . . . . . . . . . . . 71

3.15 Normalized peak optical power $(\psi)$ versus bandwidth efficiency $(\eta)$ for TC-MSM and TC-PMSM . . . . . . . . . . . . .

3.16 Normalized average optical power $(\rho)$ versus bandwidth efficiency $(\eta)$ for "best" (minimum average optical power) in-band trellis codes . . . 76 
3.17 Normalized peak optical power $(\psi)$ versus bandwidth efficiency $(\eta)$ for "best" (minimum average optical power) in-band trellis codes . . . 77

3.18 Normalized average optical power $(\rho)$ versus number of in-band carriers $(W)$ for best in-band trellis codes . . . . . . . . . 78

3.19 Normalized peak optical power $(\psi)$ versus number of in-band carriers $(W)$ for best in-band trellis codes . . . . . . . . . . 79

3.20 Four MSM symbols and the resulting symbol-by-symbol bias signal . 80

3.21 Normalized average optical power $(\rho)$ versus bandwidth efficiency $(\eta)$ for in-band trellis coding with DC detection . . . . . . . 83

3.22 Normalized peak optical power $(\psi)$ versus bandwidth efficiency $(\eta)$ for in-band trellis coding with DC detection . . . . . . . . . 84

4.1 Illustration of MSM wireless optical system using $L$ out-of-band carriers 89

4.2 The MSM wireless optical transmitter with out-of-band carriers . . 90

4.3 Block diagram of an OFDM system . . . . . . . . . . . . . . 92

4.4 Normalized average optical power $(\rho)$ versus bandwidth efficiency $(\eta)$ for real-amplitude $L=1$ out-of-band techniques . . . . . . . . 96

4.5 Normalized peak optical power $(\psi)$ versus bandwidth efficiency $(\eta)$ for real-amplitude $L=1$ out-of-band techniques $\ldots \ldots \ldots \ldots$

4.6 Normalized average optical power $(\rho)$ versus bandwidth efficiency $(\eta)$ for real-amplitude $L=4$ out-of-band techniques $\ldots \ldots \ldots \ldots$

4.7 Normalized peak optical power $(\psi)$ versus bandwidth efficiency $(\eta)$ for real-amplitude $L=4$ out-of-band techniques . . . . . . . . .

4.8 Illustration of the minimum possible amplitude between two sample points . . . . . . . . . . . . . . 106

4.9 Illustration of the maximum possible amplitude between two sample points . . . . . . . . . . . . . . . . . . 107 
4.10 Normalized average optical power $(\rho)$ versus scaling factor for TCMSM code with $W=4, U=2, K=5, M=4$ and $L=4 \ldots \ldots \ldots 111$

4.11 Normalized average optical power $(\rho)$ versus bandwidth efficiency $(\eta)$ for scaled 9-APSK $L=1$ out-of-band techniques . . . . . . . . . . . 112

4.12 Normalized peak optical power $(\psi)$ versus bandwidth efficiency $(\eta)$ for 9-APSK scaled $L=1$ out-of-band techniques . . . . . . . . . .

4.13 Normalized average optical power $(\rho)$ versus bandwidth efficiency $(\eta)$ for scaled 9-APSK $L=4$ out-of-band techniques . . . . . . . . . . 114

4.14 Normalized peak optical power $(\psi)$ versus bandwidth efficiency $(\eta)$ for scaled 9-APSK $L=4$ out-of-band techniques . . . . . . . . . . . 115

4.15 Probability distribution of normalized average optical power for all input symbols in normal MSM systems . . . . . . . . . . . .

4.16 Normalized average optical power $(\rho)$ versus bandwidth efficiency $(\eta)$ for reduced memory $L=4$ out-of-band techniques . . . . . . . . . .

4.17 Normalized peak optical power $(\psi)$ versus bandwidth efficiency $(\eta)$ for reduced memory $L=4$ out-of-band techniques . . . . . . . . . . . 123

4.18 Normalized average optical power $(\rho)$ versus bandwidth efficiency $(\eta)$ for out-of-band carriers with DC detection technique . . . . . . 125

4.19 Normalized peak optical power $(\psi)$ versus bandwidth efficiency $(\eta)$ for out-of-band carriers with DC detection technique . . . . . . . . 126

4.20 Normalized average optical power $(\rho)$ versus number of in-band carriers $(W)$ for out-of-band techniques $\ldots \ldots \ldots \ldots$

4.21 Normalized peak optical power $(\psi)$ versus number of in-band carriers $(W)$ for out-of-band techniques $\ldots \ldots \ldots \ldots$

5.1 Reduction in $\rho$ by applying various technique to a baseline normal MSM system at $\eta=1 \ldots \ldots \ldots \ldots \ldots \ldots$ 
A.1 Normalized average optical power $(\rho)$ versus bandwidth efficiency $(\eta)$ for real-amplitude $L=2$ out-of-band techniques . . . . . . . . . . 142

A.2 Normalized peak optical power $(\psi)$ versus bandwidth efficiency $(\eta)$ for real-amplitude $L=2$ out-of-band techniques . . . . . . . . . . . 143

A.3 Normalized average optical power $(\rho)$ versus bandwidth efficiency $(\eta)$ for real-amplitude $L=3$ out-of-band techniques . . . . . . . . . . 144

A.4 Normalized peak optical power $(\psi)$ versus bandwidth efficiency $(\eta)$ for real-amplitude $L=3$ out-of-band techniques . . . . . . . . . 145

A.5 Normalized average optical power $(\rho)$ versus bandwidth efficiency $(\eta)$ for scaled 9-APSK $L=2$ out-of-band techniques . . . . . . . . 146

A.6 Normalized peak optical power $(\psi)$ versus bandwidth efficiency $(\eta)$ for 9-APSK scaled $L=2$ out-of-band techniques . . . . . . . . . 147

A.7 Normalized average optical power $(\rho)$ versus bandwidth efficiency $(\eta)$ for scaled 9-APSK $L=3$ out-of-band techniques . . . . . . . . . . . 148

A.8 Normalized peak optical power $(\psi)$ versus bandwidth efficiency $(\eta)$ for scaled 9-APSK $L=3$ out-of-band techniques . . . . . . . . . . . . 149 


\section{List of Tables}

1.1 Comparison between wireless optical and RF systems for short-range

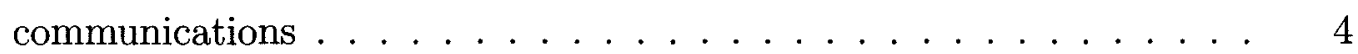

2.1 Allowable average optical power of point emitter optical source under IEC class $1[3] \ldots \ldots \ldots \ldots$. . . . . . . . . . . . . . . . 34

3.1 Outputs of trellis in Figure 3.10 given current state and inputs $b^{0}$ and $b^{1} 61$

3.2 PA Outputs of trellis in Figure 3.12 given current state and inputs $b^{0}$

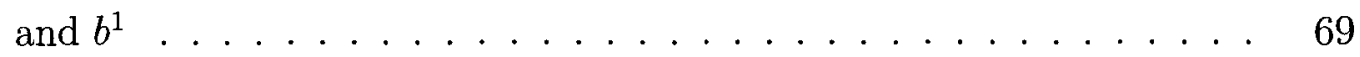

3.3 Explanation of legends used in performance figures in Chapter $3 \ldots 71$

3.4 Properties of each TC-MSM and TC-PMSM codes (assuming $d_{0}^{2}=1$ ) $\quad 74$

3.5 Properties of the "best" (minimum average optical power) in-band trellis codes (assuming $\left.d_{0}^{2}=1\right) \ldots \ldots \ldots \ldots \ldots$. . . . . . . . 77

3.6 Properties of TC-MSM codes with DC detection technique(assuming

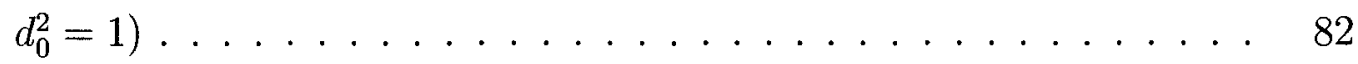

4.1 Explanation of legends used in performance figures in Chapter 4 . . 95

4.2 Performance of $L=1$ real-amplitude out-of-band carriers applied to normal MSM systems, tone injection systems and the best in-band trellis codes . . . . . . . . . . . . . . . . . . . . . 102

4.3 Performance of $L=4$ real-amplitude out-of-band carriers applied to normal MSM systems, tone injection systems and the best in-band trellis codes $\ldots \ldots \ldots \ldots \ldots \ldots \ldots \ldots$ 
4.4 Performance of $L=1$ scaled 9-APSK out-of-band carriers applied to normal MSM systems and the best in-band trellis codes . . . . . . 117

4.5 Performance of $L=4$ scaled 9-APSK out-of-band carriers applied to normal MSM systems and the best in-band trellis codes . . . . . . 118

4.6 Performance of $\mathrm{DC}$ detection technique with $L=4$ out-of-band carriers applied to the best in-band trellis codes . . . . . . . . . . . . 127

5.1 Reduction in $\rho$ and $\psi$ by applying various technique to a baseline normal MSM system at $\eta=1 \ldots \ldots \ldots \ldots \ldots$

A.1 Performance of $L=2$ real-amplitude out-of-band carriers applied to normal MSM systems and the best in-band trellis codes . . . . . . 150

A.2 Performance of $L=3$ real-amplitude out-of-band carriers applied to normal MSM systems and the best in-band trellis codes . . . . . . . 151

A.3 Performance of $L=2$ scaled 9-APSK out-of-band carriers applied to normal MSM systems and the best in-band trellis codes . . . . . . . 152

A.4 Performance of $L=3$ scaled 9-APSK out-of-band carriers applied to normal MSM systems and the best in-band trellis codes . . . . . . . 153 


\section{Chapter 1}

\section{Introduction}

\subsection{Context}

With the evolution of semiconductor technologies, the physical size and weight of electronic devices are constantly becoming smaller. Currently, portable devices such as personal digital assistants and laptop computers are commonly used. These devices enable high-speed data processing and vast data storage in a mobile package. Although such technologies are convenient, the demanding issue of mobile data exchange is yet to be addressed. Typical applications include data exchange between two terminals over relatively short distance. For example, in an indoor environment the communication between a mobile terminal and a base station could be used to provide Internet access for mobile users. Moreover, the base station could form an ad hoc network to realize data sharing between several mobile terminals in the same room. Using multiple base stations located in different rooms of the same building, an infrastructure network can be formed to connect all the mobile terminals inside the building. Such high-speed local area networks are expected to greatly improve work efficiency and therefore have attracted great attention. To address these needs, several solutions have been proposed to deliver high-speed data communication over 
a short range.

Direct connection via cables is the most simple means to establish data link between terminals. Such link is able to support stable data exchange at high speed, depending on the specification used. Examples include Universal Serial Bus (USB) [9] and IEEE1394b High Performance Serial Bus [10]. Data communication is reliable since it can be disturbed only when physical damage is found in the cables and connectors. Although this solution is simple and reliable, there are many drawbacks. The mobility of users carrying the terminals is greatly reduced. Incompatibility may exist between terminals using connectors or cables with different physical layout. The potential cost to replace broken connectors and cables due to repeated use is increased.

Communication using radio frequency $(\mathrm{RF})$ electromagnetic radiation has been studied and applied extensively [11]. This solution realizes the greatest mobile communication ability and therefore provided the most freedom for users. However, the cost of such implementation is relatively high due to the high complexity of circuit design. Frequency up-conversion and down-conversion circuits need to be designed in addition to baseband circuits. For example, a Bluetooth [12] wireless hand-free/set with organic light-emitting diode (LED) display has been designed for a portable MP3 player at the cost of US $\$ 100$ per module [13]. The design uses the Bluetooth specification $1.2[14]$ and provides data rates of $1 \mathrm{Mb} / \mathrm{s}$. Moreover, the electromagnetic spectrum is licensed under federal regulatory bodies. Fees need to be paid in order to use the desired RF spectrum, which increases system cost. RF communication also has to be contained strictly in the allocated spectral mask to prevent interference with other communication schemes. The allocated spectrum must also be utilized with care to prevent interference between multiple users in the system. This complicates transmitter design and further increases implementation cost. Finally, $\mathrm{RF}$ electromagnetic radiation is easy to intercept, which makes data transmission 
insecure. As a result, special techniques need to be employed to secure the data link.

Wireless optical communications use infrared radiation as a medium to transmit information. It can provide short-range high-speed data link at relatively low cost. A data rate of $4 \mathrm{Mb} / \mathrm{s}$ is realized over a short distance at the cost of US $\$ 1$ per module [15]. The optical transmitter is usually implemented using an LED, whose circuit design complexity is low comparing to RF transmitters. Photodiodes are often used at the receiver for detection. Typical photodiode detector areas are large compared to the wavelength of infrared radiation. This leads to spatial diversity that prevents multipath fading, which is common in RF communications [2]. Both the light emitting and light detecting components are available in high volumes at relatively low cost and have small physical size, reducing the system cost and making them easy to be installed in portable devices. The infrared spectrum is unregulated worldwide, further reducing system cost. Wireless optical communications can be easily blocked by opaque objects. Thus, interference with other ongoing communication schemes is minimized. Moreover, eavesdropping can be prevented effectively by containing the communication inside opaque boundaries. Base stations can be set up in different rooms and linked together via wired connections, forming a network inside the building as shown in Figure 1.1. These advantages make wireless optical communications an attractive solution for short range data communications. A comparison between wireless optical systems and RF systems for short-range wireless communication is shown in Table 1.1.

There are also several disadvantages to wireless optical communications. An important drawback is that the data link can only be provided in a short range such as an indoor environment since infrared light cannot penetrate opaque objects. Also, eye and skin safety requirements constraint the average optical power radiated from the optical transmitter. This limits the achievable data rate of wireless optical links. Strong noise exists in many indoor environment arising from sunlight, incandescent 


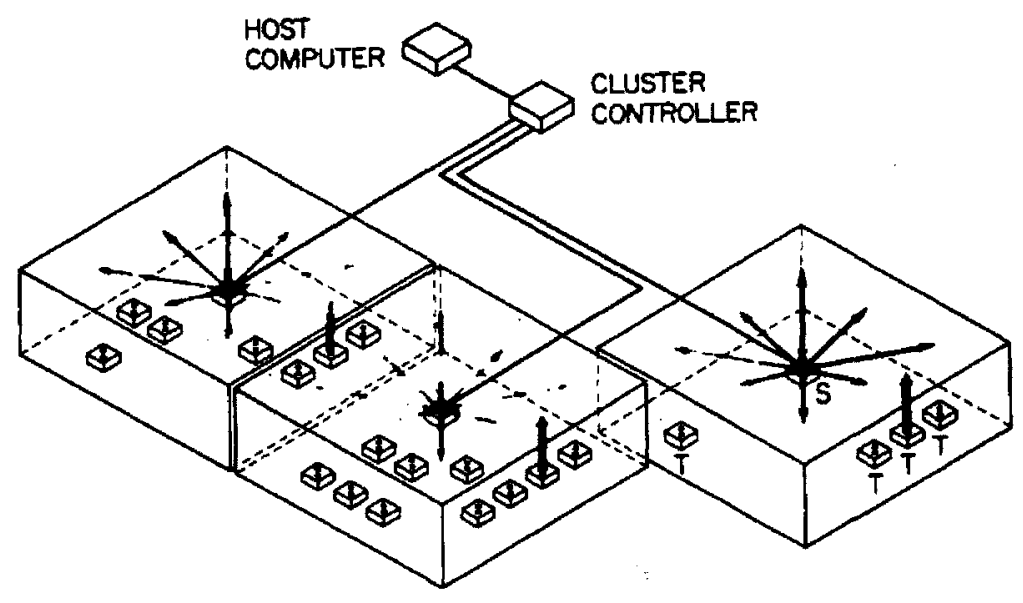

Figure 1.1: Wireless optical base stations connecting terminals inside a building and forming a network,S=base station, $\mathrm{T}=$ terminal [1]

\begin{tabular}{|l|l|l|}
\hline & $\mathrm{RF}$ & Wireless Optical \\
\hline Bandwidth & strictly restricted & unregulated worldwide \\
\hline Multipath Fading & present & absent \\
\hline Protection from eavesdropping & hard to implement & easy to implement \\
\hline Circuit Design Complexity & high & low \\
\hline Cost & expensive & cheap \\
\hline Mobility & high & medium \\
\hline
\end{tabular}

Table 1.1: Comparison between wireless optical and RF systems for short-range communications 
light and fluorescent light [2]. Such noise degrades the receiver bit error rate (BER) performance. Moreover, channel and noise measurements need to be done for different rooms to design an efficient wireless optical system. However, wireless optical communications is an attractive low-cost alternative for RF systems over a short range. This thesis investigates techniques to improve the optical power efficiency of a certain wireless optical system, namely multiple-subcarrier modulated (MSM) diffuse wireless optical systems.

To summarize, in this section we have presented several solutions for short-range wireless communications. The advantages and drawbacks of wireless optical communication systems are discussed, which justifies its use as an low-cost alternative for RF communications. In Section 1.2, current implementations of wireless optical systems are discussed. Different system architectures, proposed channel models, baseband modulation and coding schemes, industry standards and several experimental systems are presented. The MSM technique and its use in wireless optical systems are introduced. Finally, the problem of average optical power reduction for MSM diffuse wireless optical systems is briefly discussed. This problem is the focus of this thesis and is discussed in detail in Chapter 2. A similar problem in electrical MSM systems, along with its solutions and link to this thesis are presented in Section 1.3. Previous work on the average optical power reduction for wireless optical systems are discussed in Section 1.4 and the techniques developed in this thesis are briefly introduced in Section 1.5. This chapter concludes in Section 1.6 with the thesis structure. 


\subsection{Current Wireless Optical System Implementa- tions}

The work of Gfeller and Bapst [1] first investigated indoor wireless optical communications and demonstrated a working system operating at data rates of less than $1 \mathrm{Mb} / \mathrm{s}$. Since then, extensive studies has been conducted in this area. Various architectures of wireless optical communication systems including directed, diffuse, tracked and multi-spot diffusing systems have been proposed to offer different data rates with different mobility and optical power consumptions $[2,5,16]$. Channel models have been studied for both directed and diffuse infrared links to facilitate analytical analysis of the wireless optical systems $[2,17]$. Several baseband modulation and coding schemes are proposed and their optical power efficiency examined given a fixed data rate and a fixed BER. Industry standards are established and experimental systems providing data rates as high as $1 \mathrm{~Gb} / \mathrm{s}$ are presented [16]. Finally, the use of MSM in wireless optical systems is investigated and shown to provide bandwidth efficiency communications as well as multiple access [18]. An overview of these implementations are presented within this section.

\subsubsection{Wireless Optical System Architectures}

Several architectures for wireless optical links have been proposed previously with different transmitter and receiver configurations. It is convenient to classify them according to two criteria: degree of directionality of the transmitter and receiver, and whether the link relies upon the existence of an uninterrupted line-of-sight (LOS) path, as shown in Figure 1.2. Directed links use narrow-beam transmitters and receivers with narrow field-of-view (FOV), thus they have to be directed at each other 


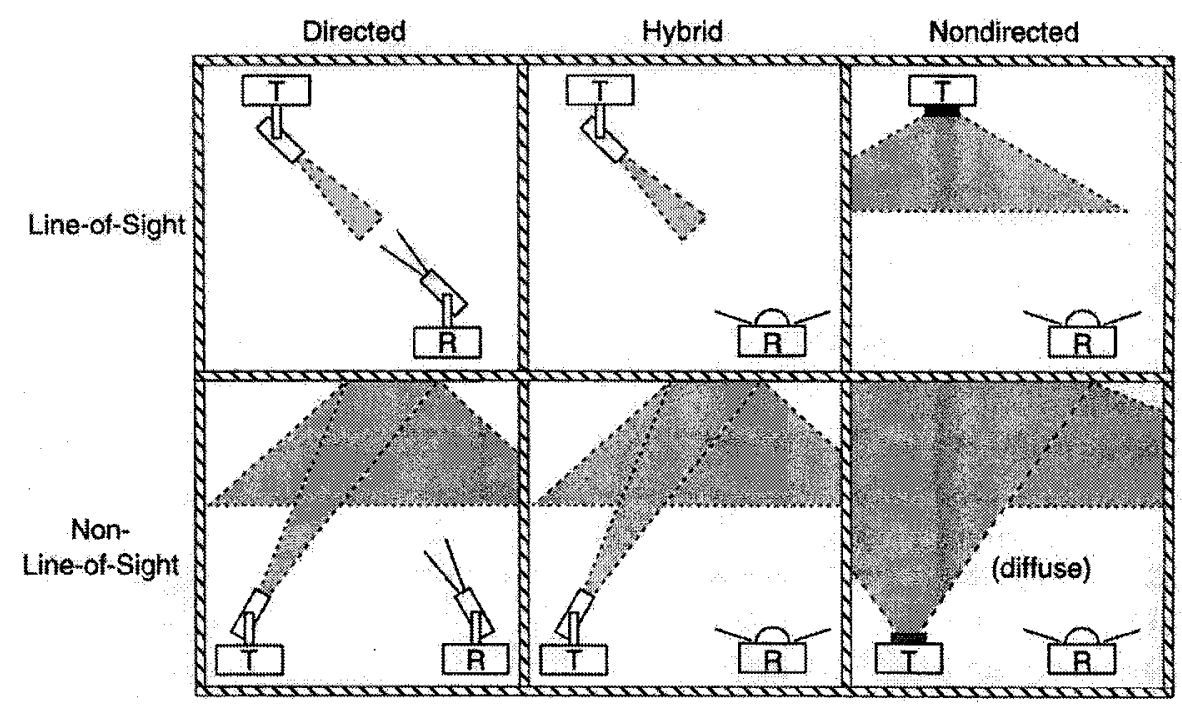

Figure 1.2: Classification of wireless optical system architectures [2]

to establish a data link. In this case, mobility of the user is greatly restricted. However, the narrow FOV reduces the ambient light noise detected by the receiver. This increases the receiver signal-to-noise ratio (SNR) and optical power efficiency of the system. On the other hand, nondirected links employ wide-beam transmitters and wide-FOV receivers and therefore do not require directionality. For such systems, user mobility is increased at the cost of reduced SNR at the receiver due to its wide FOV, which increases the detected ambient light noise and hence decreases the receiver SNR. Hybrid systems use transmiter and receivers with different types of transmitter beams and receiver FOV.

LOS wireless optical systems rely on the the existence of an uninterrupted link between the transmitter and receiver. This type of link has low multipath distortion and high power efficiency and therefore can support a higher data rate at a given power constraint. However, for LOS systems user mobility is restricted and system reliability could be compromised due to blockage of the LOS link. 


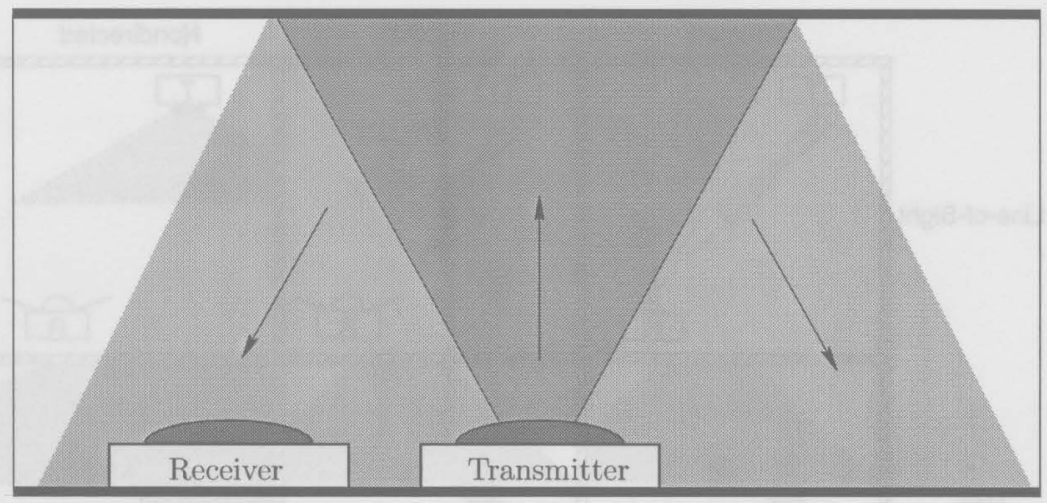

Figure 1.3: Diffuse Wireless Optical Communication System [3]

Non-LOS systems utilize multiple reflections of the transmitted signals from the ceilings and walls. Although they experience high multipath distortion and low power efficiency, they are more reliable in ensuring the existence of data link than LOS systems and can provide the most mobility for mobile terminals. The greatest robustness and ease of use are achieved by nondirected-non-LOS systems, which are often refereed to as a diffuse system [2]. An illustration of diffuse wireless optical systems is shown in Figure 1.3. A high optical power is required at the transmitter to form such links. The severe multipath distortion in such systems results in a low-pass channel response, where higher frequency components are severely degraded. These channels also suffer from a high path loss from the transmitter to the receiver. Details of the channel model will be discussed in Chapter 2.

Diffuse systems are well suited for applications where the ability to guarantee the existence of data link is more important than the data rate. For example, a security system that checks the identification of each mobile terminal inside the room. In this thesis, our optical power reduction techniques focus on diffuse systems because of the large path loss inherent in these multipath channels. With an increased optical power efficiency, diffuse systems can combine a high data rate with high robustness and 

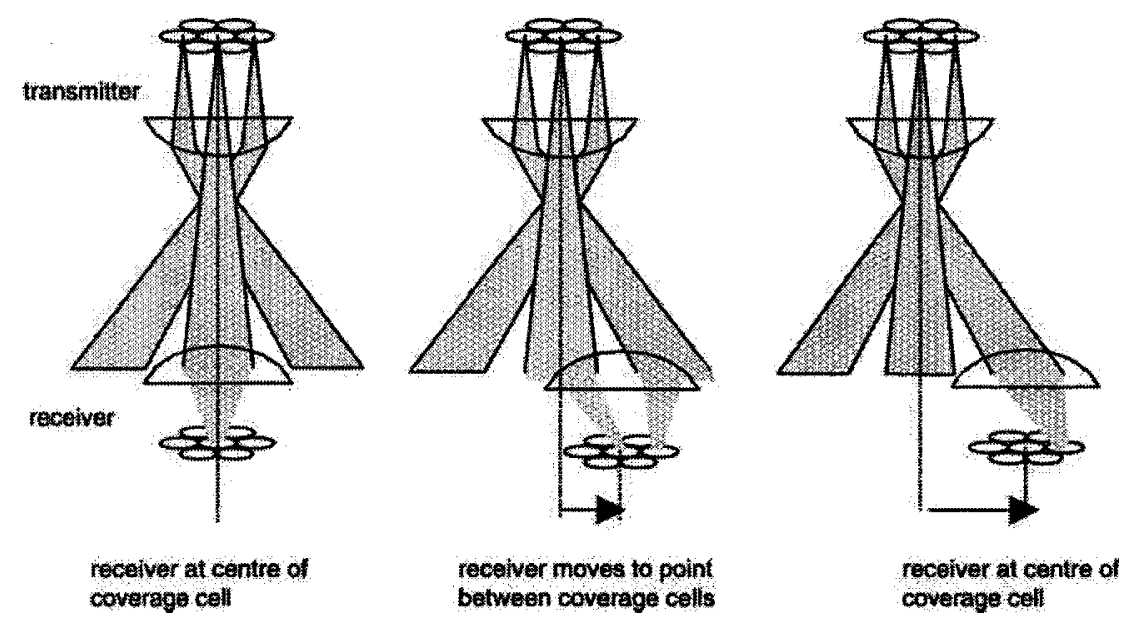

Figure 1.4: Illustration of a tracked wireless optical system [4]

high user mobility. Such systems are an attractive solution for short-range wireless communications.

Apart from the architectures discussed above, cellular tracked wireless optical systems and multi-spot diffusing configurations have also been proposed to offer both high data rate and reliable communications. Tracked wireless optical systems use directed transmitters and receivers with narrow FOV and also rely on the existence of a LOS link $[4,16,19,20]$. However, a separate tracking subsystem is designed to dynamically adjust the transmitter-receiver alignment in order to maintain the LOS link. An example of tracked system using multiple sources and detectors is shown in Figure 1.4. The area covered by the base station transmitter is divided into several cells. When the mobile terminal leaves its current cell to an adjacent cell, the base station detects the move and the base station transmitter beam is switched to the destination cell. As a result of the existence of LOS link, data rates of up to $1 \mathrm{~Gb} / \mathrm{s}$ have been reported [16].

Figure 1.5 presents a multi-spot diffusing configuration in which the transmitter sends multiple narrow beams and produces several diffusing spots on the ceiling. 


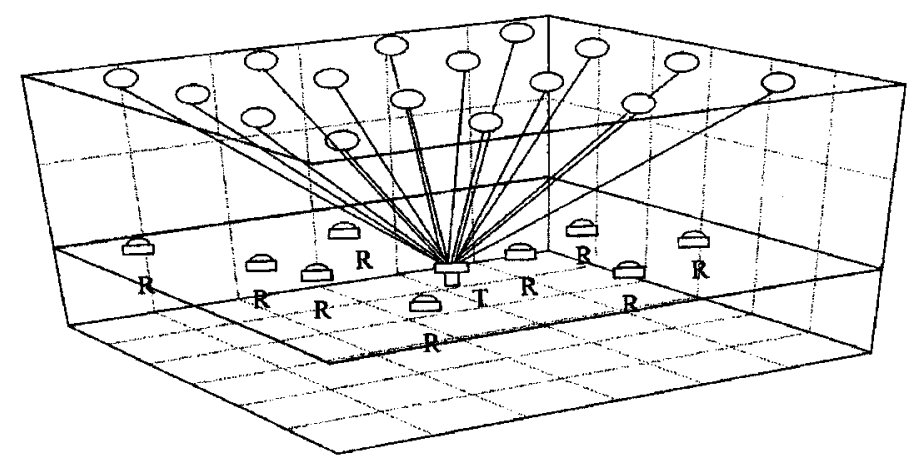

Figure 1.5: Illustration of a multi-spot diffusing configuration [5]

The same data are transmitted on all beams. The receiver could be implemented by using either a single-element wide-FOV receiver or an angle diversity receiver [2]. This configuration relies on multiple LOS paths between the diffusing spot and the receiver. As shown in [5], with a large enough number of diffusing spots $(10 \times 10)$ and an angle diversity receiver, no shadowing is observed even if there is a person standing next to the receiver. Therefore the data link is robust. Moreover, the multispot diffusing configuration has a smaller multipath distortion comparing to diffuse systems due to the multiple LOS paths. As a result, the receiver SNR is improved. By using angle diversity receiver which employs multiple narrow-FOV receiver elements, the receiver SNR can be further improved. The disadvantage of such system is the increased complexity in the design of both transmitter and receiver.

In this thesis we will focus on diffuse wireless optical systems and address its problem of low optical power efficiency. 


\subsubsection{Channel Model, Baseband Modulation and Coding Schemes}

In order to design wireless optical systems that operate well in the real world, models which accurately characterize realistic infrared channels are needed to provide reliable simulation results. In [2], a simple baseband channel is discussed for directed wireless optical systems where multipath distortion is low. For diffuse systems, multiple reflections of the transmitted signals result in high multipath distortion. To characterize such distortion, a ceiling-bounce functional model for multipath infrared channels is proposed in [17]. It is shown that the proposed model closely matches experimental results and can be realized at moderate complexity. This model is then used extensively for simulating the performance of various modulation and coding schemes.

Using the proposed channel model, various modulation schemes have been studied and simulation results given. As will be discussed in Chapter 2, intensity modulation with direct detection is widely employed in wireless optical systems. As a result the modulated electrical amplitudes must be non-negative since the transmitted signals are intensities. Additionally, the peak amplitude must be limited due to the limited dynamic range of the transmitter. Eye and skin safety regulations require that the average amplitude, i.e., optical power, is constrained. As a result of these amplitude constraints it is not possible to apply conventional electrical modulation directly to optical wireless channels.

The most simple form of modulation scheme is binary on-off keying (OOK) as shown in Figure 1.6. The symbol period is denoted as $T$. One of the two symbols is chosen depending on the input bit. The average optical power efficiency of OOK is low comparing to other modulation schemes [3]. However, due to its simple implementation, wireless optical systems using OOK are chosen as a baseline system. 

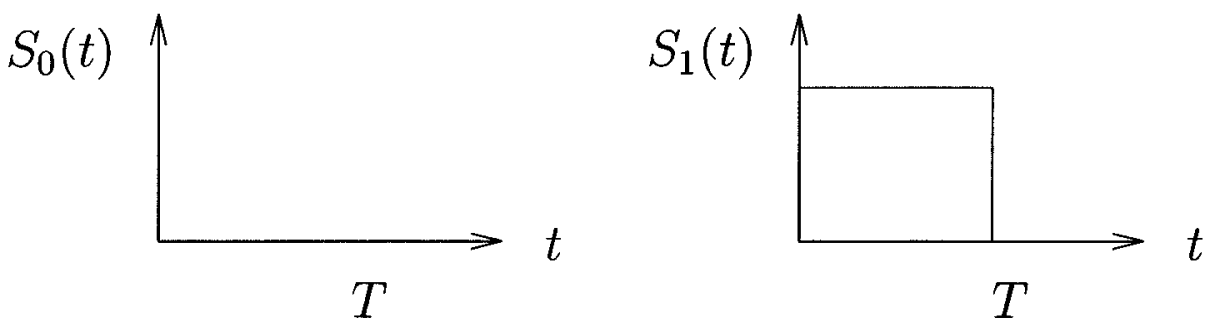

Figure 1.6: Binary On-Off Keying

The performance of any other wireless optical systems are compared to the reference OOK system operating at the same bit rate and same BER to quantify their gains. Pulse-position modulation (PPM) is a modulation scheme where information is encoded into the position of pulses, as shown in an example in Figure 1.7. The PPM modulation has a higher bandwidth requirement, but it offers lower average optical power requirement [3]. Since wireless optical communications are mostly powerlimited [3], PPM has been widely employed and is included in the IEEE Standard 802.11-1997 physical layer specification and the fast infrared (FIR) standard defined by the Infrared Data Association (IrDA) [15]. The IrDA is a nonprofit organization whose goal is to develop globally adopted specifications for infrared wireless communication [15].

To improve the average optical power efficiency, coded wireless optical systems have been proposed. Trellis-coded PPM systems have been studied in $[21,22]$ while trellis-coded multiple PPM (MPPM) systems have been studied in [23]. Since coded systems impose a structure on the sequence of output symbols, the receiver detection performance is improved and hence the average optical power efficiencies of the coded systems are increased. As a result, electrical coding gain as high as $5 \mathrm{~dB}$ has been achieved. 

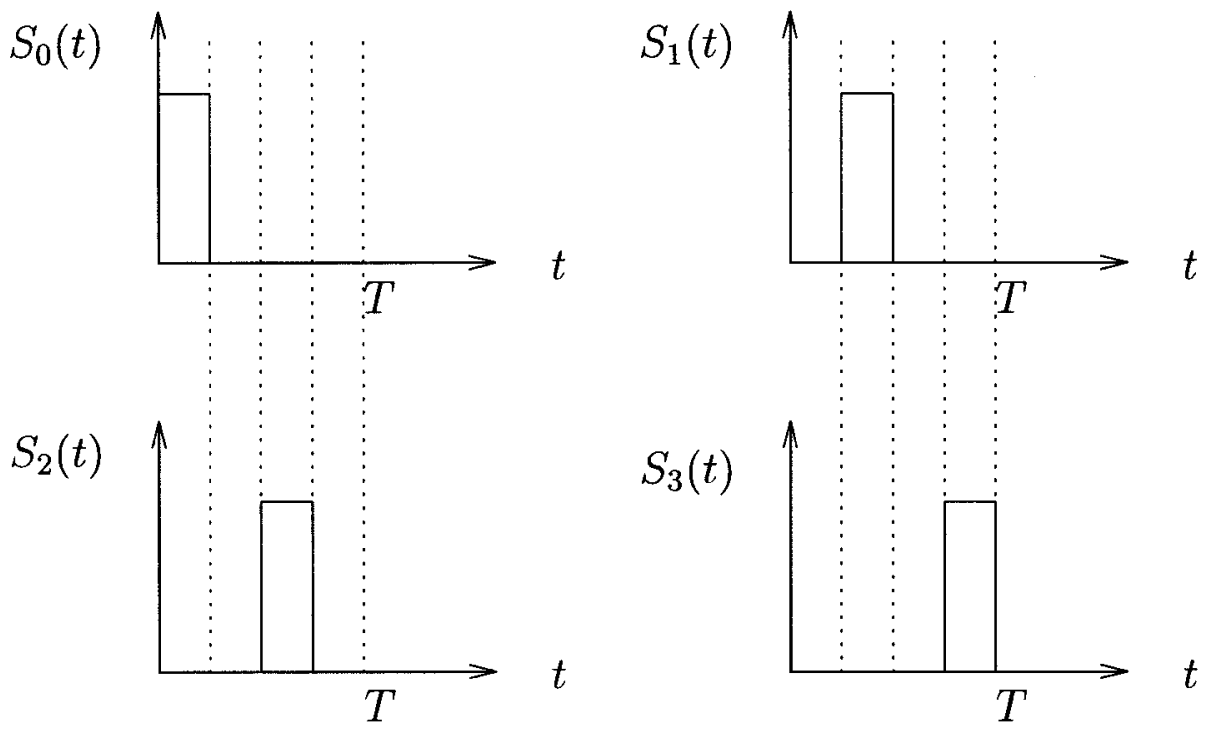

Figure 1.7: 4-PPM

\subsubsection{Experimental Links and Commercial Systems}

To verify theoretical results, experimental diffuse wireless optical systems have been built and shown to provide high data-rate communications. A $50 \mathrm{Mb} / \mathrm{s}$ diffuse infrared wireless link using OOK with decision-feedback equalization has been demonstrated and analyzed $[24,25]$. Moreover, a diffuse wireless optical system with a data rate of $100 \mathrm{Mb} / \mathrm{s}$ using OOK has been demonstated [26]. To achieve higher average optical power efficiency, 4-PPM modulation scheme with decision-feedback equalization is employed to establish a $25 \mathrm{Mb} / \mathrm{s}$ wireless optical link [27]. Experimental tracked wireless optical systems have also been demonstrated to provide data rate as high as $1 \mathrm{~Gb} / \mathrm{s}[4,16,19,20]$. Recently, a tracked system using integrated-circuit transmitter and receiver is reported and operates at $155 \mathrm{Mb} / \mathrm{s}$ [28].

The experimental systems discussed above greatly contributed to the formulation of industry standards. The IEEE standard 802.11-1997 physical layer specification specifies a $1 \mathrm{Mb} / \mathrm{s}$ wireless optical link using 16-PPM [10]. Moreover, the IrDA has 


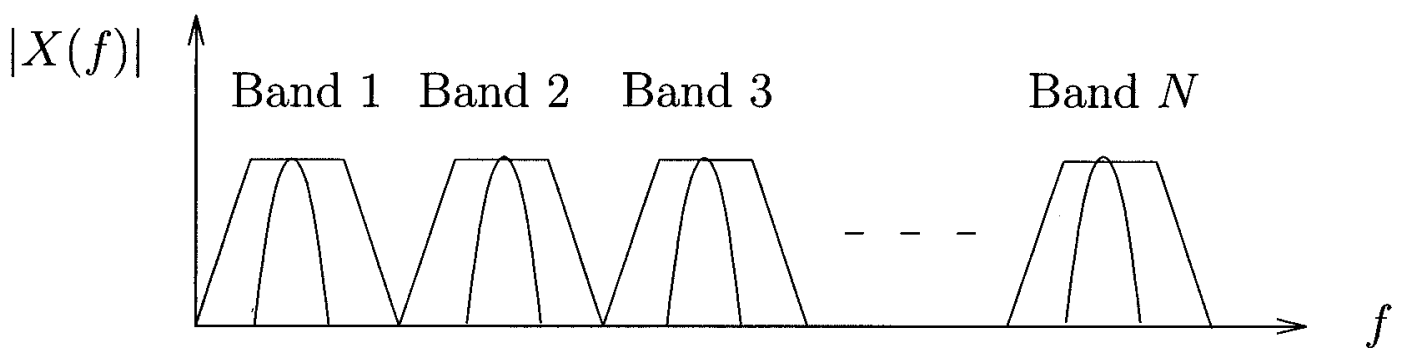

Figure 1.8: An example of multiple subcarrier modulation

specified the optical media interfaces for serial infrared (SIR) data transmission up to and including $115.2 \mathrm{~kb} / \mathrm{s}, 0.576 \mathrm{Mb} / \mathrm{s}, 1.152 \mathrm{Mb} / \mathrm{s}, 4.0 \mathrm{Mb} / \mathrm{s}$ [15]. Recent Ultra Fast Infrared (UFIR) standard under IrDA has achieved data rates up to $16 \mathrm{Mb} / \mathrm{s}$. A newer version of this standard realizing a higher data rate of $100 \mathrm{Mb} / \mathrm{s}$ is currently being developed.

\subsubsection{Multiple-Subcarrier Modulation (MSM)}

All the previously discussed modulation techniques are baseband modulation schemes without any notion of carrier. However, in electrical signaling, MSM systems have been applied extensively to perform frequency-division multiplexing as well as to combat inter-symbol interference (ISI) on frequency selective channels [29]. The frequency spectrum is divided into subbands and information-bearing carrier signals are designed in each subband, as shown in an example in Figure 1.8. Since each carrier signal is orthogonal to other carrier signals, the receiver can separate each carrier and therefore MSM systems can offer multi-user communications.

The MSM techniques have been applied on the optical fibre channels to support video distribution systems [30]. Independent information is modulated onto narrow-band electrical carriers with different frequencies. Then, the carrier signals are combined and a single laser performs the electro-optical conversion to transmit the optical signal in the fiber. At the user end the receiver performs opto-electrical 
conversion and separates each carrier signal. In this way a high aggregate data rate is achieved while each receiver operates within a narrow band.

The use of MSM in non-directed wireless optical communications was first investigated by Carruthers and Kahn [18] as a means to provide bandwidth efficient communication as well as for multi-access. Although wireless optical communications are more power-limited, for non-directed links the high multipath distortion results in a low-pass channel, whose bandwidth is also limited. The power efficiencies of MSM wireless optical systems are compared at $30 \mathrm{Mb} / \mathrm{s}$ and $100 \mathrm{Mb} / \mathrm{s}$ over an ensemble of experimentally determined multipath channels. It is shown that MSM wireless optical systems achieve a higher data rate than single-carrier wireless optical systems without equalization and are more bandwidth efficient.

Other advantages of MSM wireless optical systems include the capability to design carriers where channel attenuation and noise power are low. In this way the receiver SNR is improved. The spectrum of a rectangular OOK system, for example, has a high tail power and is therefore very susceptible to multipath-induced ISI. In contrast, by designing multiple narrow-band carriers in the same bandwidth, the transmitted signal power is contained to the bandwidth of the channel. As a result, greater immunity to ISI in diffuse wireless optical channels is achieved by avoiding the use of channel frequencies which highly distort the transmitted signal.

The drawbacks of MSM wireless optical systems are its high average and peak optical power requirements. As will be discussed in Chapter 2, the average and peak optical power are proportional to the minimum and maximum amplitude of the electrical MSM signal respectively. Therefore it is intuitively clear that the addition of many carrier signals results in high average and peak optical power requirements for MSM wireless optical systems. Moreover, the constraint on the average optical power is much tighter than the constraint on the peak optical power. As a result the problem of average optical power reduction for MSM wireless optical systems has 
McMaster University - Dept. Elec. \& Comp. Eng.

attracted great attention.

Previously, much work has been done for average optical power reduction in MSM wireless optical systems [31-34]. The reduction reported is at best $1 \mathrm{~dB}$ optical at the cost of using real-numbered carrier amplitudes [32] and is achieved when comparing at the same bandwidth efficiency (defined in Chapter 2) versus a reference system. In this thesis, novel methods are proposed and the reduction is as high as $2.63 \mathrm{~dB}$ optical. Moreover, as will be discussed in Chapter 2, every $1 \mathrm{~dB}$ of optical power reduction is equivalent to $2 \mathrm{~dB}$ of electrical power reduction. Thus the techniques developed in this thesis achieved as high as $5.26 \mathrm{~dB}$ of electrical power reduction.

In conclusion, current wireless optical implementations are presented in this section. The MSM wireless optical systems and its problem of high average optical power are discussed. This problem is similar to the problem of high peak-to-average power ratio (PAPR) in electrical MSM systems. In Section 1.3, previous work on the reduction of PAPR in electrical MSM systems as well as the differences of the two problems are presented. As a result of these differences, it is necessary to develop novel techniques rather than to apply the electrical PAPR reduction techniques directly to wireless optical systems.

\subsection{Electrical MSM PAPR Reduction}

The PAPR of a signal over a certain duration of time is defined as the ratio between its squared maximum amplitude and the time average of the squared signal. Similar to MSM wireless optical systems, the addition of many sinusoids in electrical MSM systems leads to a high PAPR. The problem of PAPR reduction in electrical MSM systems has attracted great attention and many techniques have been proposed. A comprehensive review of these techniques is available in the book by Jose Tellado [35] and is briefly discussed in this section to provide relevant background. 
Theoretical analyses have been done previously to formulate the problem of PAPR reduction and establish bounds of the PAPR of a given modulation and coding scheme. In [36] a lower bound on the PAPR of a constant energy code (e.g., phase shift keying) is given as a function of its rate, distance and length. The lower bound of achievable rates of a code given its distance and PAPR is also derived. Moreover, in [37] a bound for the peak of the continuous envelope of the signals was proposed based on the maximum of its corresponding oversampled sequence.

The simplest way for PAPR reduction is to set a threshold at the transmitter and clip the peaky signal exceeding the threshold. It is shown in [38-41] that clipping increases the BER at the receiver and widens the spectrum of the transmitted signal. The widened spectrum may violate the spectral mask and cause interference with other communication schemes. Therefore, methods to reduce the effect of spectral widening due to clipping have also been proposed [42-45]. Moreover, for MSM symbols with a PAPR exceeding a pre-defined threshold, the amplitudes on all carriers are scaled down to reduce the PAPR [46].

Another class of methods achieve a PAPR reduction at the cost of reduced transmission rate rather than a reduction in BER at the receiver. This class of methods apply coding to avoid use of MSM symbols with a high PAPR. Different types of codes have been used, including Golay complementary sequences [43, 47], Reed-Muller codes [48-50] and cyclic codes [51]. Finally, given the total number of carriers, an exhaustive search is performed on the set of all MSM symbols to choose a subset of symbols with low PAPR and/or good distance properties [52-55]. These methods achieve PAPR reduction at the cost of computational burden to find good codes. When a large number of carriers are used, the search may become impractical to implement.

Other methods include adding a phase-rotating vector to change the phase of 
transmitted symbols in order to reduce the PAPR [56-61] and constellation shaping [35,62]. Additionally, reserved subcarriers are added and their amplitudes determined by either an iterative algorithm using FFT and IFFT [63] or a standard optimization algorithm [64]. These reserved subcarrier signals reduce the PAPR of electrical waveform at the cost of increased electrical power and bandwidth requirement, which are very limited in RF systems. Parallel-combinatory multiple-subcarrier (PCMS) adds a set of subcarriers with zero amplitude and uses their position to convey additional information while reducing the PAPR [65]. Input bits are mapped to each carrier using a fixed mapping and recovered using uncoded symbol-by-symbol detection.

The survey presented in this section is by no means complete. Significant improvements have been reported for PAPR reduction in electrical MSM systems. In the book by Tellado [35], a method named tone injection has been proposed and is included in Very high bit-rate Digital Subscriber Line (VDSL) standard [66]. This method increases the constellation size by adding a vector whose real part or imaginary part have absolute value $D$. Therefore each original constellation point can be mapped to several points in the expanded constellation. The value $D$ is chosen such that the minimum distance of the expanded constellation is the same as the original constellation, as shown in Figure 1.9. In this way, detection performance at the receiver is not affected. The receiver simply performs a modulo- $D$ operation to map the received constellation points back to the original constellation. By changing one constellation point for each MSM symbol over all carriers, this technique achieves $5 \mathrm{~dB}$ reduction in the PAPR when 256 carriers are used.

Although the techniques discussed in this section achieved significant reduction in PAPR for electrical MSM systems, their direction application in MSM wireless optical systems does not guarantee good reduction in average optical power. In fact, the tone injection method is applied in MSM wireless optical systems in Chapter 3 


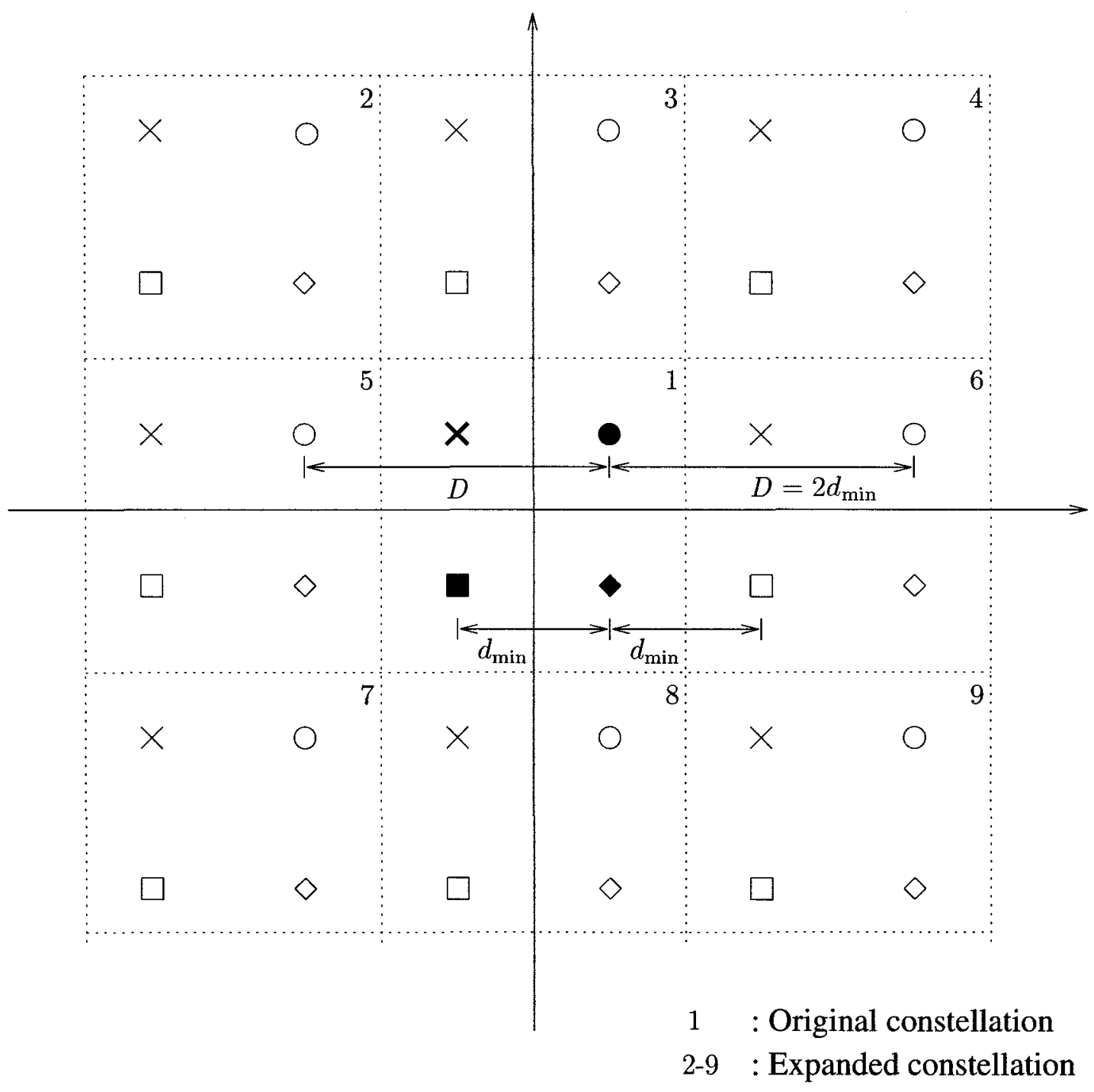

Figure 1.9: Illustration of the expanded constellation of the tone injection method 
and it is shown that the reduction in average optical power is $0.66 \mathrm{~dB}$ optical at best by changing one constellation point for each MSM symbol over all carriers.

The reason why the direct application of electrical PAPR reduction techniques are not guaranteed to perform well is because of the amplitudes constraints set by wireless optical systems. First, as will be presented in Chapter 2, the electrical signal before electro-optical conversion in wireless optical systems must be non-negative for all time. Second, the average optical power of wireless optical systems is proportional to the minimum amplitude of the electrical signal, while electrical PAPR is related to the envelope of the squared electrical signal. The difference in objective function as well as the amplitude non-negativity constraint require special design for average optical power reduction in MSM wireless optical systems.

In conclusion, techniques for PAPR reduction in electrical MSM systems are presented in this section. Although applying these techniques directly in MSM wireless optical systems does not guarantee good average optical power reduction, the ideas presented here provide insight into the design of efficient average optical reduction techniques. In Section 1.4, previous work on the average optical power reduction for MSM wireless optical systems are presented. A brief introduction of the techniques developed in this thesis are discussed in Section 1.5 and the thesis structure is given in Section 1.6.

\subsection{Average Optical Power Reduction Techniques for MSM Wireless Optical Systems}

In order to reduce the average optical power as well as to improve detection, the use of a time-varying bias signal has been proposed [67] in wireless optical systems. As opposed to a fixed bias level, the time-varying bias signal depends on the transmitted 
MSM signal. Moreover, the detection of this symbol-by-symbol bias provides a degree of signal space diversity, which improves the BER performance at the receiver.

In MSM wireless optical systems, the problem of average optical power reduction was first treated by You [31]. Block codes were developed to minimize the average optical power. The design of block codes requires an exhaustive search and is therefore impractical for large number of carriers. Average optical power reduction up to $0.1 \mathrm{~dB}$ optical is achieved over a conventional MSM wireless optical system employing QPSK modulation and a symbol-by-symbol bias at the same bandwidth efficiency. Also, the peak optical power is increased on the order of $3 \mathrm{~dB}$ optical by employing this technique. As will be discussed in Chapter 2, a large peak optical power may violate eye and skin safety requirements and cause non-linear distortion at the transmitter.

Other average optical power reduction techniques have also been considered. In [32], a single reserved subcarrier is added to the in-band signal and the transmitted amplitude optimized to provide the minimum average optical power when BPSK modulation is employed. In the case of more reserved subcarriers a suboptimal search procedure was employed. The PCMS technique [65] was applied to MSM wireless optical systems by simply adding a symbol-by-symbol bias [33]. In [34], the subcarrier signal-point sequence (SSPS) is introduced. A group of codewords were chosen from a subset of MSM signals with low average optical power such that the minimum distance among each pair of codewords is maximized. Notice, that all previous work for average power reduction of MSM optical wireless systems considered block coding and did not code over sequences of output signals. Additionally, the reduction of both peak and optical powers was not treated in any previous work.

In Section 1.5, the methods proposed in this thesis are briefly introduced and shown to achieve significant average optical power reduction, which exceeds previous work. Moreover, reduction in both average and peak optical powers is also realized. 
McMaster University - Dept. Elec. \& Comp. Eng.

\subsection{Research Direction}

The goal of this research is to investigate techniques to reduce the average optical power requirement in diffuse MSM wireless optical systems. In this thesis three techniques are developed to address this problem. In Chapter 3 in-band trellis coding technique is proposed. This technique expands the signal set as in [65] and designs trellis codes over the increased degrees of freedom. An average optical power reduction of $0.95 \mathrm{~dB}$ with a simultaneous peak power reduction of $0.44 \mathrm{~dB}$ over conventional MSM systems at the same bandwidth efficiency are achieved. It should be noted that although the average optical powers at all bandwidth efficiencies are always reduced, the peak optical powers are sometimes increased. However, the increase in peak optical power is at most $0.57 \mathrm{~dB}$ optical and it can be considered that this technique does not greatly affect the peak optical power. The cost of this technique is the additional complexity in the form of a trellis encoder at the transmitter and a Viterbi decoder at the receiver.

The second technique exploits the unregulated bandwidth in wireless optical channels and designs out-of-band carrier signals to reduce the average optical power. An average optical power reduction of $2.56 \mathrm{~dB}$ optical is realized at the expense of 4 out-of-band carriers with real-numbered in-phase and quadrature amplitudes and an increase in the peak optical power as high as $3.96 \mathrm{~dB}$ optical. This reduction in average optical power far exceeds those of the previous works comparing at the same bandwidth efficiency. This technique requires the transmitter to generate realnumbered amplitudes, which can be difficult to implement. Moreover, the design of out-of-band carriers are performed once and stored in a lookup table. Therefore additional memory is required at the transmitter, which will be discussed in detail in Chapter 4.

To alleviate transmitter complexity, an alternative solution is developed in which 
out-of-band carrier amplitudes are chosen from a discrete constellation. In this manner, a $1.50 \mathrm{~dB}$ of average optical power reduction is achieved with a peak optical increase of $1.30 \mathrm{~dB}$ using 4 out-of-band carriers. Notice the reduction in average optical power is still significant (equivalent to $3 \mathrm{~dB}$ electrical power reduction), while the increase in peak optical is greatly reduced. Moreover, this technique requires less memory at the transmitter. In applications where the additional memory must be tightly limited, out-of-band carriers can be designed for a portion of the data symbols with high average optical power requirement. It is shown in Chapter 4 that the percentage of loss in average optical power reduction and the percentage of savings in memory requirements are roughly the same.

The third technique detects the symbol-by-symbol bias at the receiver. This received DC level provides a degree of diversity in the signal space and thus improves detection. In this manner, up to $0.50 \mathrm{~dB}$ of average optical power reduction is achieved together with a peak power reduction of $0.46 \mathrm{~dB}$. The performance of this technique depends on the channel at lower frequencies. If strong noise power is found at lower frequencies near DC, such as a strong fluorescent light noise discussed in Chapter 2, the performance of this technique will be degraded.

Finally, the techniques developed in this work can be employed individually or combined. For example, the real-amplitude out-of-band system which achieves significant average optical power reduction is combined with the in-band trellis coding system and DC detection technique, both of which has the peak optical power reduction capability. The resulting system achieved $2.63 \mathrm{~dB}$ of average optical power reduction with a peak optical power increase of $1.73 \mathrm{~dB}$. This is equivalent to $5.26 \mathrm{~dB}$ of electrical power reduction and far exceeds previous results. 


\subsection{Thesis Structure}

This chapter has introduced wireless optical communication systems. The problem of average optical power reduction for MSM wireless optical systems is identified and a brief discussion on the techniques developed in this thesis is given. In Chapter 2, details of the channel model and MSM wireless optical systems are discussed. The amplitude constaints of wireless optical systems as well as the definition of average and peak optical power and bandwidth efficiency are given. A baseline MSM wireless optical system is defined and the metrics used to compare average and peak optical powers of different techniques are presented.

Chapter 3 discusses the use of trellis coding and DC detection technique for average and peak optical power reduction in MSM wireless optical systems. Chapter 4 presents the design of out-of-band carrier signals. The performance of these techniques are quantified using the metrics introduced in Chapter 2. The thesis concludes in Chapter 5 with a summary and discussion of simulation results as well as directions for future work. 


\section{Chapter 2}

\section{Channel Model}

Knowledge and modeling of wireless optical channels are critical in the design of power efficient MSM systems. This chapter introduces the basic channel configurations in wireless optical channels. Commonly used transmitter and receiver devices are also presented. The noise and distortion arising from various causes are discussed and their impact on system design studied. Eye and skin safety regulations impose a tight constraint on the average optical power. Finally, the modeling of wireless optical channels and MSM wireless optical systems are presented. Figures of merit for comparing average and peak optical power efficiencies of various wireless optical systems are also explicitly established.

\subsection{Basic Channel Structure}

In electrical signalling, the amplitude, frequency or phase of the electrical signal can be modulated to carry information [68]. However, in a low-cost wireless optical system it is extremely difficult to directly modulate the frequency or phase of the optical signal [3]. As a result, for wireless optical channels the most practical modulation and demodulation schemes are intensity modulation and direct detection. An illustration 


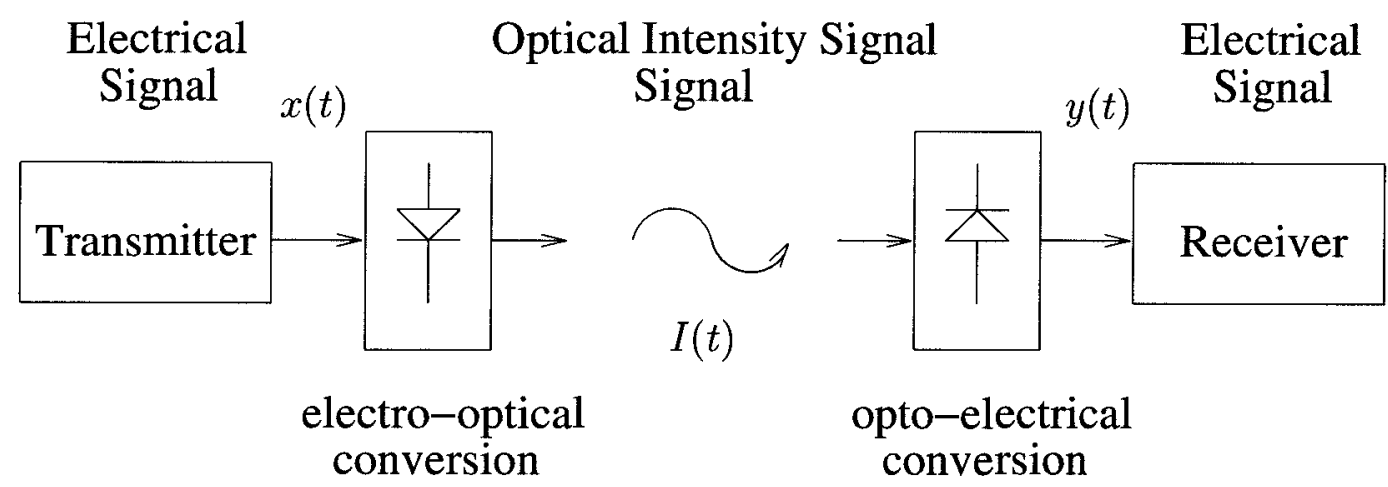

Figure 2.1: Intensity modulated, direct detection wireless optical channel [3]

of this channel is given in Figure 2.1 [3].

The source information is encoded in the electrical waveform $x(t)$. Then $x(t)$ is modulated as the instantaneous intensity of the optical signal $I(t)$, which is proportional to $x(t)$. This electro-optical conversion is termed optical intensity modulation and can be done using low-cost electronic devices such as a light emitting diode (LED). LEDs are commonly used as optical transmitter due to their low cost and low circuit design complexity. They are also considered eye-safe because they are an incoherent light source and emit light over a large surface area. However, there are several drawbacks. Typical low cost LEDs have low modulation banwidth limited to hundreds of $\mathrm{MHz}$. Their spectral width is broad, leading to wide receiver optical passband and therefore a higher noise power due to the wide-spectrum background light. LEDs also have low electro-optic conversion efficiencies of $10 \%-20 \%$, where electro-optic conversion efficiency is defiend as the ratio of the total emitted optical power divided by the total input electrical power.

In order to detect the modulated intensity, a photodiode is used at the receiver. The photodiode integrates the received optical intensity over its area and produces a proportional electrical current $y(t)$. This conversion from the optical domain to the electrical domain is termed direct detection. Commercial large-area photodiodes 
are manufactured using silicon technologies and are available at low cost only at the near-infrared band of 780-950 $\mathrm{nm}$ [2]. Therefore, most commercial wireless optical links operates in the 780-950 $\mathrm{nm}$ band.

The optical signal propagates through free-space, possibly undergoing multiple reflections from the ceilings and walls in an diffuse configuration. Therefore similar to $R F$ channels, the received signal is the sum of the transmitted signal and its delayed versions. In the electrical domain when the time delay of two signals cause a 180 degree phase shift, the two signals would cancel each other and cause spectral nulls in the channel frequency response. This effect is termed multipath fading [68] and it degrades the receiver BER performance in electrical RF systems. However, the photodiode receiver implementation of wireless optical systems provides immunity to multipath fading. The typical size of the light detector in photodiodes is approximately $1 \mathrm{~cm}^{2}$ [3], while the wavelength of the infrared light is 780-950 nm. Therefore the size of the light detector with respect to the wavelength of infrared light is relatively large, on the order of $10^{4}$ times of the wavelength. Moreover, different versions of the received signal are spaced on the order of half a wavelength apart [3]. As a result the photodiode receives a huge number of signals with different delays. This provides a degree of spatial diversity at the receiver and mitigates multipath fading effects [68].

Although multipath fading is not a major impairment to IM/DD wireless optical systems, multipath distortion severely degrades receiver performance. The summation of a large number of received signals with different delay time causes inter-symbol interference (ISI), which degrades the receiver BER performance [68]. The effect of multipath distortion is most noticeable in non-directed systems where the receiver relies upon the reflected signals. In this case, the channel often exhibits low-pass characteristics. Directed, tracked and multi-spot diffusing systems, on the other hand, are less affected by multipath distortion due to the existence of a LOS path, which 
ensures the presence of a strong unreflected transmitted signal [2].

To characterize the channels for indoor non-directed wireless optical systems, experimental studies have been performed. A vector network analyzer and a $832 \mathrm{~nm}$ optical transmitter were employed to measure the channel frequency response [6]. The room under measurement was an empty conference room shown in Figure 2.2(a). For all measurements the receiver was placed at desk height and pointed straight up. To form a non-directed LOS system, the transmitter is placed near the ceiling and pointed straight down. To form a diffuse system, the transmitter is placed at desk height and pointed straight up. For both systems the unshadowed and shadowed configurations are examined, where shadowing is introduced by having a person stand next to the receiver. The measured frequency magnitude response $|H(f)|$ of both configurations under both systems are shown in Figure 2.2(b).

It can be seen that for unshadowed diffuse system a decrease in the channel magnitude response is found at higher frequencies. This is explained by the high multipath distortion it experiences arising from multiple reflections of the transmitted signal. Unlike RF channels where specular reflections dominate, infrared signals are diffusely reflected in such systems, resulting in a continuous distribution of reflected angles [1]. As a result, the channel impulse response has a continuous pulse shape rather than discrete components. The channel magnitude response for unshadowed LOS system is nearly flat due to the presence of a strong unreflected transmitted signal. The magnitude response of shadowed channels are less predictable than unshadowed channels. However, shadowing greatly degrades the magnitude response of LOS system since the LOS path is blocked. On the other hand, diffuse systems rely on the multiple reflections of the transmitted signal and is therefore less affected by shadowing than LOS system did.

To summarize, this section presents the basic channel structure of wireless optical systems. The widely-employed IM/DD techniques are discussed along with their 


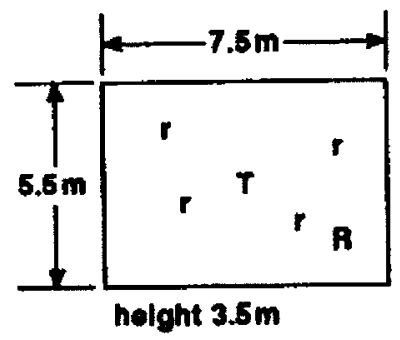

(a)

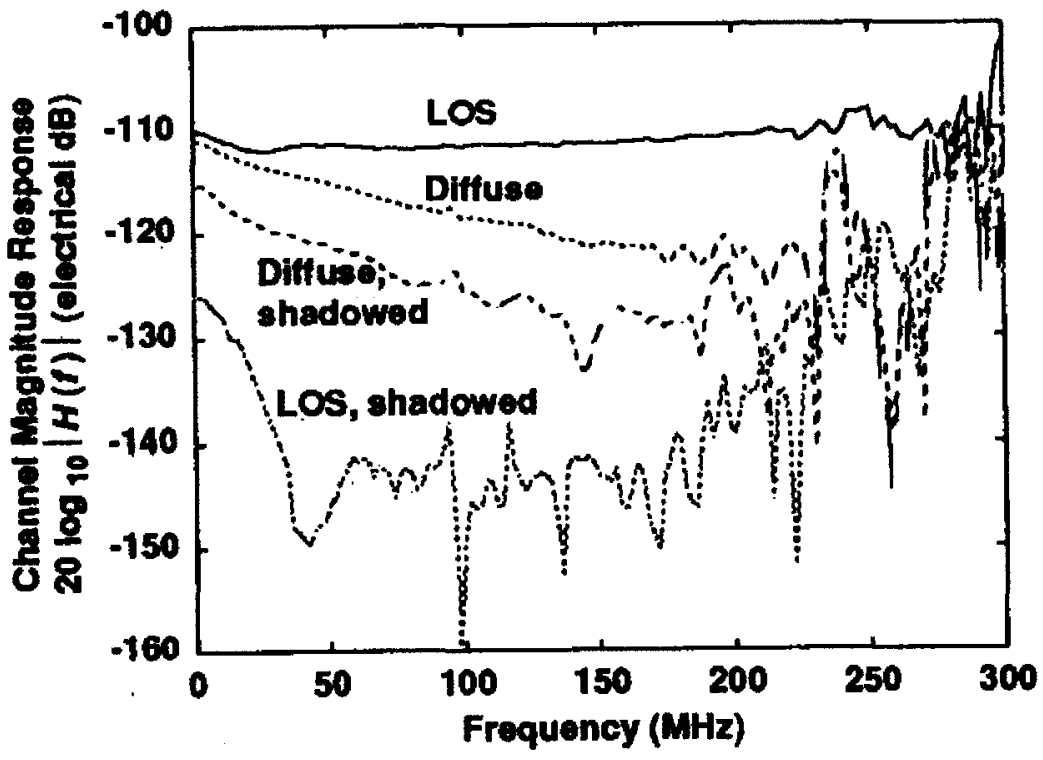

Figure 2.2: (a) Top view of the measured room. $\mathrm{T}$ is the transmitter location. Receiver is located at $\mathrm{R}$ and additional measurements have been performed at locations r. (b) Measured channel magnitude response. [6] 
implementation using LEDs and photodiodes. The channel characteristics including the effect of multipath are presented. Finally, channel measurement results for a typical room are shown and are in agreement with the analysis.

\subsection{Noise}

Apart from multipath distortion, noise is another major factor which limits the communication rate. Typical noise sources in electrical communication systems are thermal noise arising from the random motion of electrons in a conductor and shot noise arising from the current flow in several devices such as diodes and transistors [69]. Electrons move randomly and independently in a conductor and the their summation forms the current flowing through the conductor. As a result, the thermal noise is formed by the summation of many independent noise sources. According to the central limit theorem, the thermal noise can be modeled as Gaussian distributed with zero mean in the limit. Shot noise, on the other hand, arises due to the discrete nature of the current flow in diodes and transistors. The electrons are emitted at random times and the total number of electrons emitted in an interval can be modeled as having a Poisson distribution, which is non-negative [69].

For indoor wireless optical systems, the two major noise sources are the shot noise resulting from background light and receiver preamplifier noise [2]. The random motions of electrons in the preamplifier results in a thermal noise generated independentally of the received signal. Therefore, it can be modeled as Gaussian distributed and signal-independent.

The most common background light sources in wireless optical systems are sunlight, incandescent lamps and fluorescent lamps. As shown in Figure 2.3, these background lights have a wide frequency range, including the $780-950 \mathrm{~nm}$ band where the receiver photodiode operates. While optical filtering can be used to remove lights 


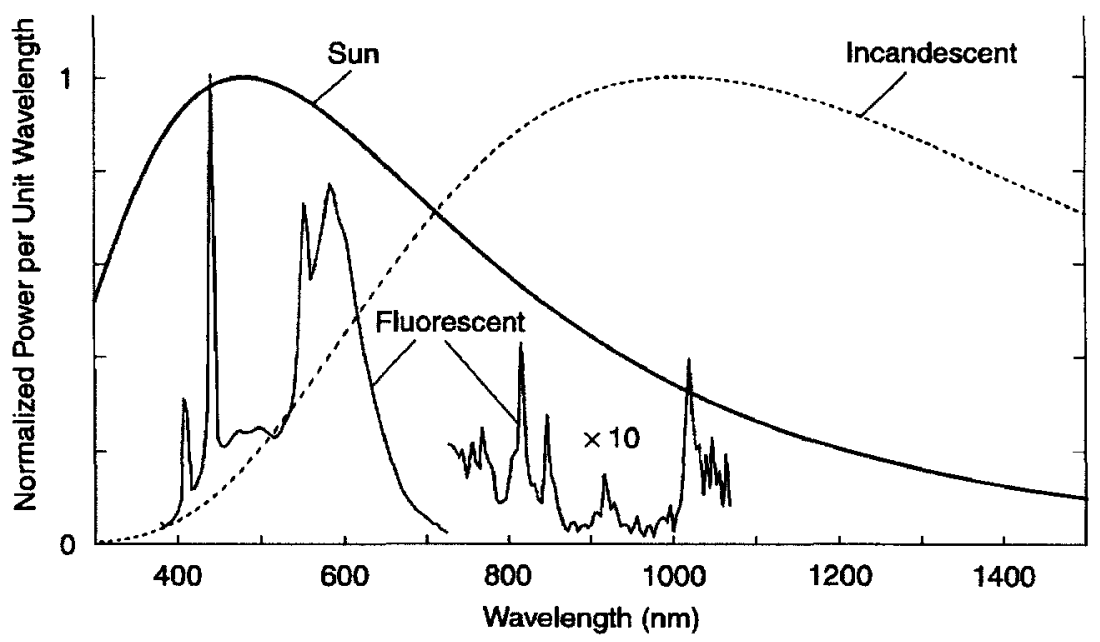

Figure 2.3: Optical power spectra of common background light sources. Spectra have been scaled to have the same maximum value. [2]

outside the photodiode band, background lights inside the $780-950 \mathrm{~nm}$ band are still detected by the photodiode. As a result, a shot noise is introduced in the photodiode current which is independent of the received signal.

In typical wireless optical channels, the shot noise resulting from background light is a dominant noise source [3]. It is formed by the summation of a large number of independent, Poisson distributed non-negative noise sources. Therefore in the limit, this shot noise can be modeled as white, Gaussian, and signal-independent according to central limit theorem. The bias resulting from the summation of these random variables can be ignored at the receiver since it is common to both the signal and the noise. Thus, a zero-mean Gaussian-distributed model of the noise is a good one [2].

Traditional fluorescent lamps are driven by power-line frequency at $50 \mathrm{~Hz}$ or $60 \mathrm{~Hz}$. Hence, the detected electrical signal spectrum contains energy at these low frequency components near DC. Harmonics of these drive frequencies up to tens of kilohertz are also present in the electrical signal spectrum [7]. Recently, newly introduced "electronic ballasts" drive fluorescent lamps at frequencies at $22 \mathrm{KHz}$ or $45 \mathrm{KHz}$. Thus detected electrical signal spectrum contains energy up to hundreds of kilohertz [7]. 


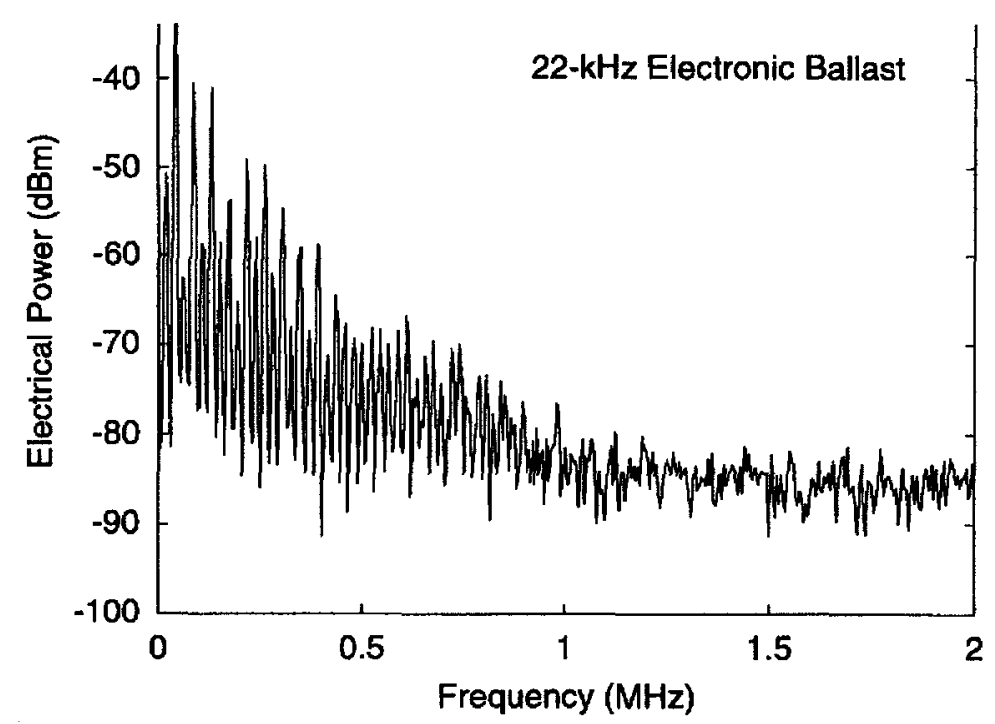

Figure 2.4: Detected electrical power spectrum of infrared emission from a fluorescent lamp driven by a $22 \mathrm{KHz}$ electronic ballast [7]

As seen in Figure 2.4, noise power concentrates at the $22 \mathrm{KHz}$ drive frequency and its harmonic frequencies. The fluorescent light energy at such frequencies may degrade detection performance of the desired signal, depending on modulation scheme employed. However, MSM wireless optical systems use multiple narrow-band carriers to transmit information. By carefully designing carrier frequencies and using fast Fourier transform (FFT) at the receiver to obtain amplitudes send on each carrier frequency, the spectrum region where fluorescent light noise power can be avoided and the effect of fluorescent light noise on MSM wireless optical system can be minimized.

In conclusion, due to the high-intensity of ambient illumination, the noise of indoor wireless optical channels is well modeled as being white, signal-independent and Gaussian distributed [2]. Additionally, when fluorescent light noise is present the channel also have a low-frequency noise at the driving frequency and its harmonic frequencies. 


\subsection{Amplitude constraints}

As seen in Figure 2.1, in intensity-modulated wireless optical systems the instantaneous intensity of the transmitted optical signal $I(t)$ is proportional to the amplitude of the electrical signal $x(t)$. Since the intensity signal $I(t)$ must be non-negative for all $t$, the electrical signal $x(t)$ must also be non-negative for all $t$. This amplitude nonnegativity constraint is not seen in electrical systems and is the most fundamental constraint in wireless optical communications.

Moreover, since infrared radiation is propagated in free-space, safety for the human body must be considered. Regulations regarding eye and skin safety impose tight constraints on the average optical power. Since the average of optical intensity signal $I(t)$ is proportional to the average of the electrical signal $x(t)$, this means a constraint is placed on the average amplitude of $x(t)$.

The International Electrotechnical Commission (IEC) is a global organization that prepares and publishes international standards for all electrical, electronic and related technologies. The IEC classifies exposure limits of optical sources into three major classes [70]. Among them, class 1 operation has the tightest constraint and therefore is the safest operation class. Devices classified as class 1 have no warning labels and can be used without special safety precautions. Among all the specifications in class 1 , the allowable exposure limit (AEL) sets a limit on the average transmitted power of the optical source. Since human skin and eyes can be damaged by heating, the average optical power of the source must be constrained. The AEL depends on the wavelength of the source, the geometry of the emitter and the intensity of the source. Table 2.1 gives the AEL of a class 1 optical source assuming a point emitter is used [3].

It is evident from Table 2.1 that the allowable average optical power increases as the optical wavelength increases. It is therefore desirable for a wireless optical 


\begin{tabular}{|l|l|l|l|l|}
\hline & $\begin{array}{l}650 \mathrm{~nm} \\
\text { visible }\end{array}$ & $\begin{array}{l}880 \mathrm{~nm} \\
\text { infrared }\end{array}$ & $\begin{array}{l}1310 \mathrm{~nm} \\
\text { infrared }\end{array}$ & $\begin{array}{l}1550 \mathrm{~nm} \\
\text { infrared }\end{array}$ \\
\hline Class 1 & $<0.2 \mathrm{~mW}$ & $<0.5 \mathrm{~mW}$ & $<8.8 \mathrm{~mW}$ & $<10 \mathrm{~mW}$ \\
\hline
\end{tabular}

Table 2.1: Allowable average optical power of point emitter optical source under IEC class $1[3]$

system to operate at the higher wavelength due to the higher average optical power budget. However, most commercial wireless optical systems operate at the $780 \mathrm{~nm}-$ $950 \mathrm{~nm}$ near-infrared band due to the availability of large-area low-cost photodiodes. As a result, a tight constraint on the average optical power is imposed on wireless optical systems. Therefore the average amplitude of the electrical signal $x(t)$ is tightly constrained.

Apart from the average optical power, the peak optical power must also be limited for human safety considerations. The other reason is that the dynamic range of the analog transmitter circuits is limited. If the maximum amplitude of the electrical signal $x(t)$ exceeds the dynamic range of the transmitter amplifier, clipping will occur and the transmitted signal will be distorted. This will affect the receiver BER performance. Therefore, the peak optical power and the maximum amplitude of $x(t)$ must be constrained.

In conclusion, the amplitude of the electrical signal $x(t)$ must satisfy the nonnegativity constraint due to the use of intensity modulation. Moreover, the average and peak amplitudes must also be limited due to human safety requirements and the limited dynamic range of the transmitter circuits. However, the constraint on the average optical power is much tighter than the constraint on the peak optical power and is therefore the main consideration in the design of wireless optical systems. 


\subsection{Channel Modeling}

Indoor wireless optical channels can be modeled as the baseband linear system [2]

$$
y(t)=r I(t) \otimes h(t)+n(t)
$$

where $\otimes$ represents convolution, $r[\mathrm{~A} / \mathrm{W}]$ is the photodetector responsivity, $I(t)[\mathrm{W}]$ is the input intensity-modulated signal, $h(t)$ is the channel impulse response and $y(t)[\mathrm{A}]$ is the received photocurrent. Without loss of generality, $r$ can be assumed to be 1 for all wireless optical systems considered. The channel frequency response $H(f)$ is

$$
H(f)=\int_{-\infty}^{+\infty} h(t) e^{-j 2 \pi f t} d t
$$

and it is often modeled as low-pass due to multipath distortion.

In this thesis, we denote the low-pass region of the spectrum as in-band and information-bearing signals are sent within this bandwidth. Moreover, in our MSM wireless optical systems we assume the channel frequency response is flat at each of the narrow-band carrier frequencies. This is a conventional model for MSM indoor wireless optical systems [31] and serves as a good starting point for signalling design. All the higher frequencies are termed out-of-band and the signals sent on the out-ofband spectral region are distorted severely. As discussed earlier in Section 2.2, the noise $n(t)$ is modeled as being white, signal independent and Gaussian distributed with zero mean and variance $\sigma^{2}$.

Since $x(t)$ is modulated onto an instantaneous optical power signal, it must satisfy

$$
x(t) \geq 0
$$

Due to eye safety requirements, the average optical power $P_{a}$ of wireless optical system must be limited. The maximum allowable average optical power is defined as $\mathbb{P}_{a}$ according to the AEL. From the channel model (2.1), a constraint is placed on the 
average amplitude of $x(t)$

$$
P_{a}=E[x(t)] \leq P_{\operatorname{amax}}
$$

rather than the power $E\left[x^{2}(t)\right]$ as in electrical channels. Thus, a reduction of electrical power in decibels is equivalent to half of that value in optical power. For example, a $3 \mathrm{~dB}$ electrical power gain corresponds to $1.5 \mathrm{~dB}$ optical gain [3].

Moreover, the peak optical power $P_{p}$ must be constrained due to the limited dynamic range of the transmitter and safety considerations. Define the maximum allowable peak optical power as $\mathbb{P}_{p}$, the constraint on the maximum amplitude of $x(t)$ can be written as

$$
P_{p}=\max _{t} x(t) \leq P_{\mathrm{pmax}} .
$$

As seen in equations (2.3), (2.4) and (2.5) all the constraints are placed on the amplitudes of $x(t)$. As discussed in Section 1.3 of Chapter 1, these amplitude constraints in wireless optical systems prohibit direct application of electrical PAPR reduction techniques. Novel methods need to be developed for average optical power reduction in wireless optical systems.

\subsection{Signal Space Concepts}

Consider a collection of $M$ signals which lie in a vector space. According to the Gram-Schmidt orthogonalization procedure, the $M$ signals can be represented as a linear combination of $N$ orthonormal basis functions, where $N \leq M$. If an $N$ dimensional Euclidean space is formed with axes being the $N$ orthonormal basis functions, then each of the $M$ signals can be represented geometrically in this space. This $N$-dimensional Euclidean space is called a signal space. Note that in the signal space, the squared Euclidean distance between any signal and the origin is the energy of that signal. 
For example, the set of signals $\left\{s_{i}(t)\right\}, i=1,2, \ldots M$ can be represented as

$$
s_{i}(t)=\sum_{j=1}^{N} s_{i j} \phi_{j}(t), \quad i=1,2, \ldots M, \quad 0 \leq t \leq T
$$

where $T$ is the symbol period and the $\phi_{j}(t), j=1,2, \ldots N$ are a set of orthonormal functions satisfying

$$
\int_{0}^{T} \phi_{i}(t) \phi_{j}(t) d t= \begin{cases}1 & \text { if } i=j \\ 0 & \text { if } i \neq j\end{cases}
$$

The first condition means that the energy of the orthonormal functions over its defined period of time is unity. The second condition means that the functions are orthogonal to each other.

The coefficients $s_{i j}$ are given by the projection of $s_{i}(t)$ onto orthonormal functions $\phi_{j}(t)$

$$
s_{i j}=\int_{0}^{T} s_{i}(t) \phi_{j}(t) d t
$$

Therefore each $s_{i}(t)$ can be determined and represented by the vector

$$
\overrightarrow{s_{i}}=\left[s_{i 1}, s_{i 2}, \ldots s_{i N}\right], \quad i=1,2, \ldots M
$$

The signal constellation is defined as the collection of all signal vectors. The signal space concept can be illustrated by an example of quadrature amplitude modulation (QAM). In $M$-QAM the $M$ signals are represented by in-phase basis function $\phi_{I}(t)$ and quadrature basis function $\phi_{Q}(t)$ defined as

$$
\begin{aligned}
\phi_{I}(t) & =\sqrt{\frac{2}{T}} \cos \left(\frac{2 \pi}{T} t\right) \operatorname{rect}\left(\frac{t}{T}\right) \\
\phi_{Q}(t) & =\sqrt{\frac{2}{T}} \sin \left(\frac{2 \pi}{T} t\right) \operatorname{rect}\left(\frac{t}{T}\right)
\end{aligned}
$$

where

$$
\operatorname{rect}\left(\frac{t}{T}\right)= \begin{cases}1 & \text { if } 0 \leq t \leq T \\ 0 & \text { otherwise }\end{cases}
$$




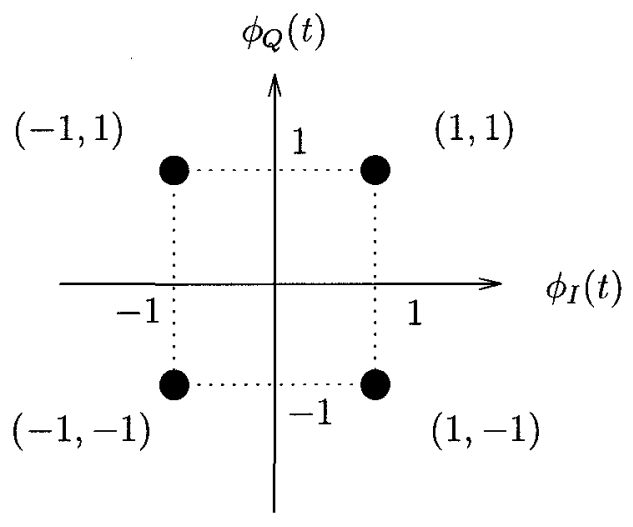

Figure 2.5: Constellation for 4-QAM (QPSK)

Therefore we have $N=2$ and

$$
s_{i}(t)=s_{i I} \phi_{I}(t)+s_{i Q} \phi_{Q}(t), \quad i=1,2, \ldots M, \quad 0 \leq t \leq T
$$

For example, the constellation for 4-QAM, also known as quadrature phase shift keying (QPSK), is shown in Figure 2.5.

The receiver projects the received signal $y(t)$ onto orthonormal functions $\phi_{j}(t)$,

$$
y_{j}=\int_{0}^{T} y(t) \phi_{j}(t) d t
$$

The resulting vector

$$
\vec{y}=\left[y_{1}, y_{2}, \ldots y_{N}\right]
$$

is used to represent $y(t)$ in the signal space.

Under the assumption that the noise $n(t)$ is Gaussian and signal-independent and that the channel frequency response is flat at each in-band carrier frequencies in MSM wireless optical systems, the channel for each in-band carrier is an ideal additive white Gaussian noise (AWGN) channel. If equiprobable signalling is used, then the optimal receiver which maximizes the probability of a correct decision is a maximum likelihood receiver, which maximizes the likelihood function defined as

$$
\operatorname{Pr}\left(\vec{y} \mid \overrightarrow{s_{i}}\right)
$$




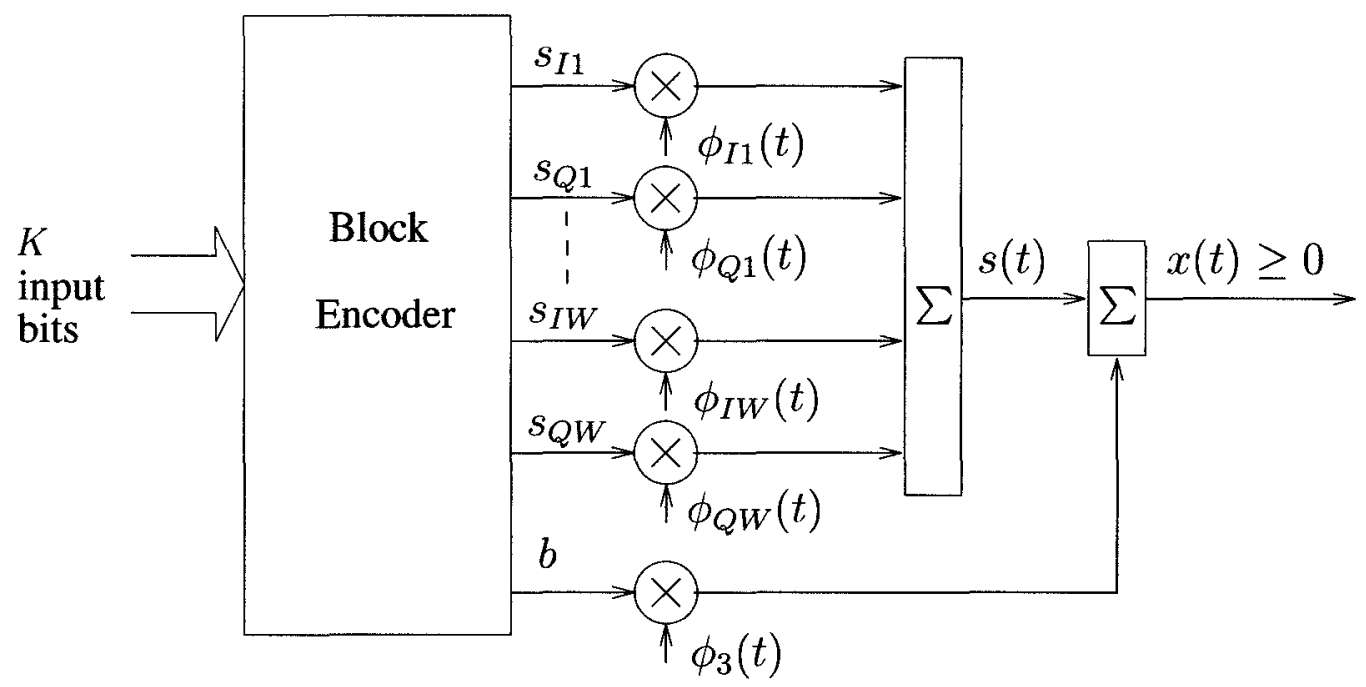

Figure 2.6: Conventional MSM wireless optical transmitter

where $\operatorname{Pr}(\cdot)$ denotes the probability of an event. The maximum likelihood receiver can be implemented either using correlators or matched filters. It selects the message point $\overrightarrow{s_{i}}$ which is closest in Euclidean distance to $\vec{y}$ in the signal space as the detection result.

\subsection{MSM Wireless Optical Channels}

The MSM wireless optical systems effectively use the channel by designing narrowband carriers where the channel attenuation and noise power are low. It also provides multiple access capability. Figure 2.6 presents a diagram of a conventional MSM wireless optical transmitter with $W$ in-band carriers. An information-bearing signal is transmitted on each of the $W$ in-band carriers. It is assumed that the symbol period is limited to $T$ seconds and that a rectangular pulse shape $\operatorname{rect}(t / T)$ is employed. Moreover, we consider a symbol-by-symbol bias in this thesis since it offers significant optical power reduction $[31,67]$. The required bias level is computed at each symbol interval to ensure the non-negativity of the electrical signal $x(t)$. 


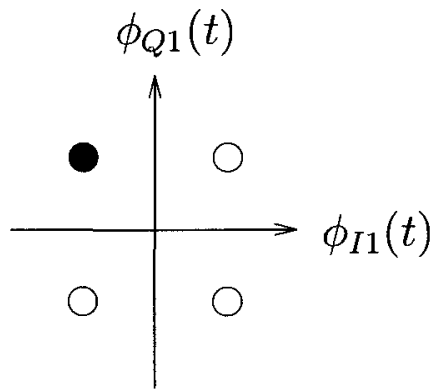

$\omega_{1}$

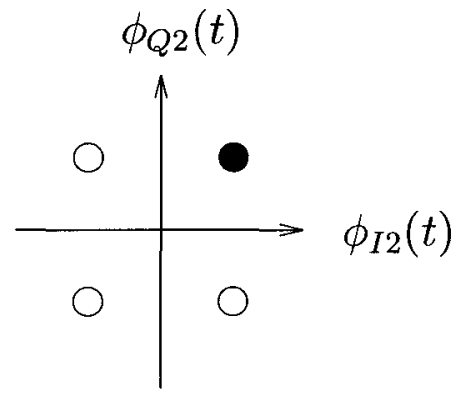

$\omega_{2}$

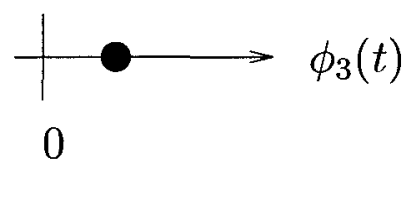

$\mathrm{DC}$

Figure 2.7: Two-carrier MSM constellation with QPSK employed on each carrier and a symbol-by-symbol bias

Under such modulation scheme, at each carrier frequency $\omega_{i}=2 \pi i / T, i \in[1, W]$ a two-dimension signal space is spanned by the unit-energy orthonormal basis functions $\phi_{I i}(t)$ and $\phi_{Q i}(t)$, defined as

$$
\begin{aligned}
\phi_{I i}(t) & =\frac{2}{\sqrt{T}} \cos \left(\frac{2 \pi i}{T} t\right) \operatorname{rect}\left(\frac{t}{T}\right) \\
\phi_{Q i}(t) & =\frac{2}{\sqrt{T}} \sin \left(\frac{2 \pi i}{T} t\right) \operatorname{rect}\left(\frac{t}{T}\right)
\end{aligned}
$$

Moreover, the symbol-by-symbol bias forms another dimension in the signal space, whose unit-energy basis function is defined as

$$
\phi_{3}(t)=\frac{1}{\sqrt{T}} \operatorname{rect}\left(\frac{t}{T}\right)
$$

Note that the orthonormal basis functions for information-bearing carriers are different at each carrier frequency $\omega_{i}, i \in[1, W]$. Therefore there are $2 W+1$ orthonormal basis functions in total. An example of MSM constellation with $W=2$ carriers and QPSK employed on each carrier is shown in Figure 2.7

Next we analyze the waveform at the $j$-th symbol interval $t \in[j T,(j+1) T)$. Let $s_{I i}^{(j)}$ and $s_{Q i}^{(j)}, i=1,2, \ldots W$ denote the projection of the transmitted signal onto 
$\phi_{I i}(t-j T)$ and $\phi_{Q i}(t-j T)$ respectively, then

$$
\begin{aligned}
s_{I i}^{(j)} & =\int_{j T}^{(j+1) T} x(t) \phi_{I i}(t-j T) d t \\
s_{Q i}^{(j)} & =\int_{j T}^{(j+1) T} x(t) \phi_{Q i}(t-j T) d t
\end{aligned}
$$

Moreover, let $b^{(j)}$ denote the projection of the transmitted signal at the $j$ th symbol period onto $\phi_{3}(t-j T)$, given by

$$
b^{(j)}=\int_{j T}^{(j+1) T} x(t) \phi_{3}(t-j T) d t
$$

The addition of $W$ information-bearing sinusoids at the $j$-th symbol interval is given by

$$
s^{(j)}(t)=\sum_{i=1}^{W}\left(s_{I i}^{(j)} \phi_{I i}(t-j T)+s_{Q i}^{(j)} \phi_{Q i}(t-j T)\right), \quad t \in[j T,(j+1) T)
$$

It is evident that this signal has zero time average and a minimum amplitude which is less than zero. In order to ensure the amplitude non-negativity constraint in equation (2.3), a bias signal must be added. For the $j$-th symbol interval $t \in[j T,(j+1) T$, the symbol-by-symbol bias $b^{(j)}$ is defined as

$$
b^{(j)}=-\min _{t \in[j T,(j+1) T)} s^{(j)}(t)
$$

The resulting electrical MSM waveform $x(t)$ can then be written as

$$
\begin{aligned}
x(t) & =\sum_{j}\left(s^{(j)}(t)+b^{(j)} \phi_{3}(t-j T)\right) \\
& =\sum_{j}\left[\sum_{i=1}^{N}\left(s_{I i}^{(j)} \phi_{I i}(t-j T)+s_{Q i}^{(j)} \phi_{Q i}(t-j T)\right)+b^{(j)} \phi_{3}(t-j T)\right]
\end{aligned}
$$

The average optical power of the MSM wireless optical system is therefore

$$
P=E[x(t)]=E\left[s^{(j)}(t)\right]+E\left[b^{(j)}\right]=E\left[b^{(j)}\right]
$$


where $E[\cdot]$ denotes the time average. Thus, the goal of an optical power reduction technique is to reduce the magnitude of the negative peak of $s(t)$ in each symbol period.

Note that the symbol-by-symbol bias $b^{(j)}$ is correlated to the transmitted signal at the $j$ th symbol period. Therefore it is clear that $b^{(j)}$ gives information about the transmitted symbols. As will be discussed in Chapter 3, this correlated information is exploited to provide a degree of signal space diversity, which improves detection of the transmitted signal.

The conventional MSM wireless optical receiver is shown in Figure 2.8. A low-pass filter (LPF) removes the signals outside the in-band region. At the $j$-th symbol interval, the receiver produces an estimate $\hat{s}_{I i}^{(j)}$ and $\hat{s}_{Q i}^{(j)}, i=1,2, \ldots W$ of the transmitted symbol over the $W$ in-band carriers. The received signal $y(t)$ at the $j$-th symbol period is projected onto each orthonormal functions $\phi_{I i}(t-j T)$ and $\phi_{Q i}(t-j T)$ to obtain

$$
\begin{aligned}
& \hat{s}_{I i}^{(j)}=\int_{j T}^{(j+1) T} y(t) \phi_{I i}(t-j T) d t \\
& \hat{s}_{Q i}^{(j)}=\int_{j T}^{(j+1) T} y(t) \phi_{Q i}(t-j T) d t
\end{aligned}
$$

This operation is usually implemented by a matched filter matched to the pulse shape in (2.15) and (2.16).

The main drawback of MSM wireless optical systems is the high average optical power requirement. The average optical power increases greatly as $W$ increases due to the addition of a large number of sinusoids. This justifies research in average optical reduction techniques for MSM wireless optical systems. 


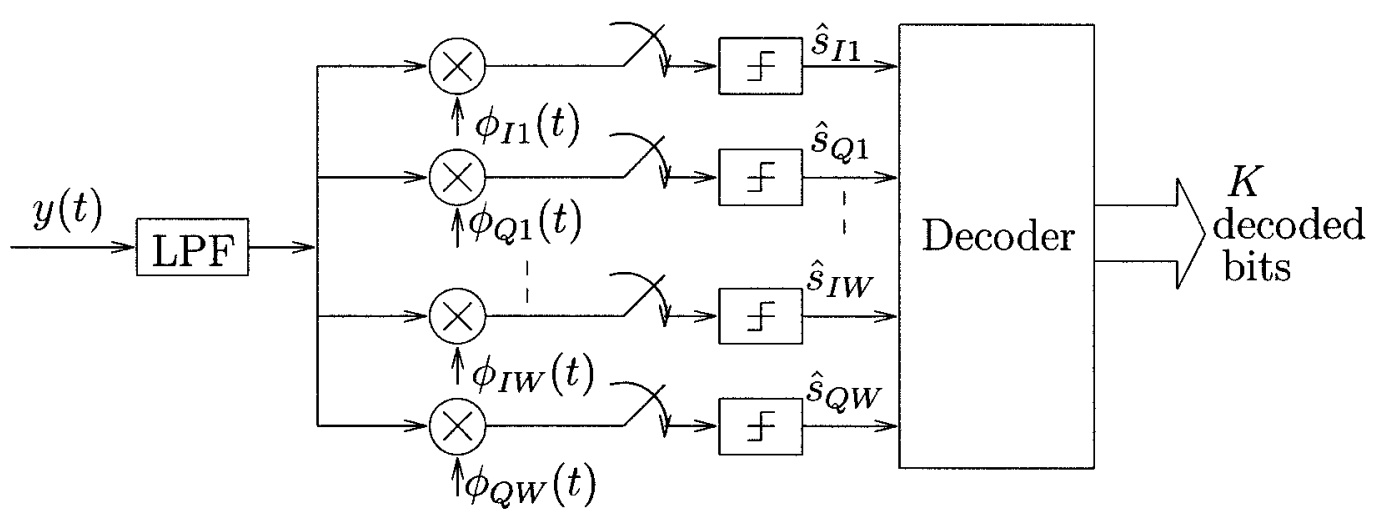

Figure 2.8: Conventional MSM wireless optical receiver

\subsection{Figures of Merit}

In this section, we define various figures of merit, namely (1) bandwidth efficiency $\eta$, (2) normalized average optical power $\rho$ and (3) normalized peak optical power $\psi$ in order to compare the peak and average optical power efficiencies of different MSM wireless optical systems.

The rectangular pulse defined earlier,

$$
\operatorname{rect}\left(\frac{t}{T}\right)= \begin{cases}1 & \text { if } 0 \leq t \leq T \\ 0 & \text { otherwise }\end{cases}
$$

has a Fourier transform given by

$$
T \operatorname{sinc}\left(\frac{\omega T}{2}\right) e^{-j \omega \frac{T}{2}}=\frac{\sin (\omega T / 2)}{\omega / 2} e^{-j \omega \frac{T}{2}}
$$

As shown in Figure 2.9, the spectrum have a null-to-null bandwidth of $4 \pi / T$.

In order to choose carrier frequencies $\omega_{i}$ which are mutually orthogonal, we can consider both "dense" packing and "coarse" packing, described by the equations below and illustrated in Figure 2.10. 

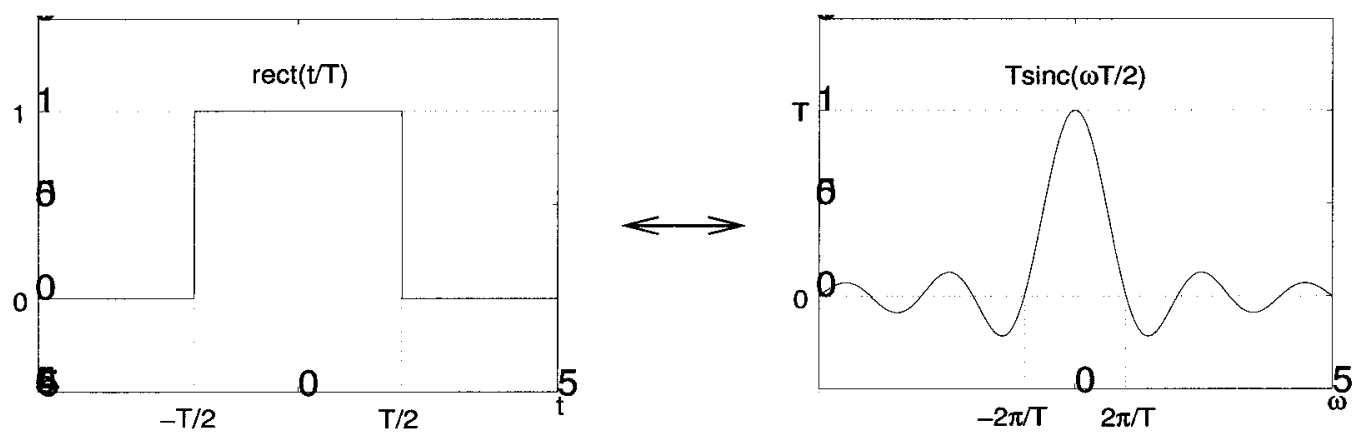

Figure 2.9: Time-domain rectangular pulse and its frequency-domain sinc pulse

$$
\begin{aligned}
\omega_{i}=\frac{2 \pi i}{T} & \text { (dense packing) } \\
\omega_{i}=\frac{4 \pi i-2 \pi}{T} & \text { (coarse packing) }
\end{aligned}
$$

Using dense packing, a bandwidth of $(W+1) \times 2 \pi / T$ is need for a total number of $W$ carriers, as opposed to $2 W \times 2 \pi / T$ in the case of coarse packing. Therefore, we consider dense packing in this thesis since it offers orthogonality at a lower bandwidth requirement. For a given bandwidth, increasing $W$ increases the data sent in each symbol. However, decoding latency is increased due to a larger symbol period. Assuming $K$ bits are sent per symbol period, the null-to-null bandwidth efficiency of our MSM wireless optical system is defined as the ratio between the bit rate and the bandwidth in natural frequency,

$$
\eta=\frac{K \times 1 / T}{(W+1) \times 1 / T}=\frac{K}{W+1} \quad[\mathrm{bits} / \mathrm{sec} / \mathrm{Hz}] .
$$

The bandwidth efficiency measures the data rate of a communication system normalized to its bandwidth requirement. For MSM systems, the bandwidth efficiency characterizes both the gain achieved in data rate and the increase in bandwidth requirement by using multiple carriers. Therefore, it is a better metric than the data rate when comparing MSM systems to single-carrier systems or baseband systems. 

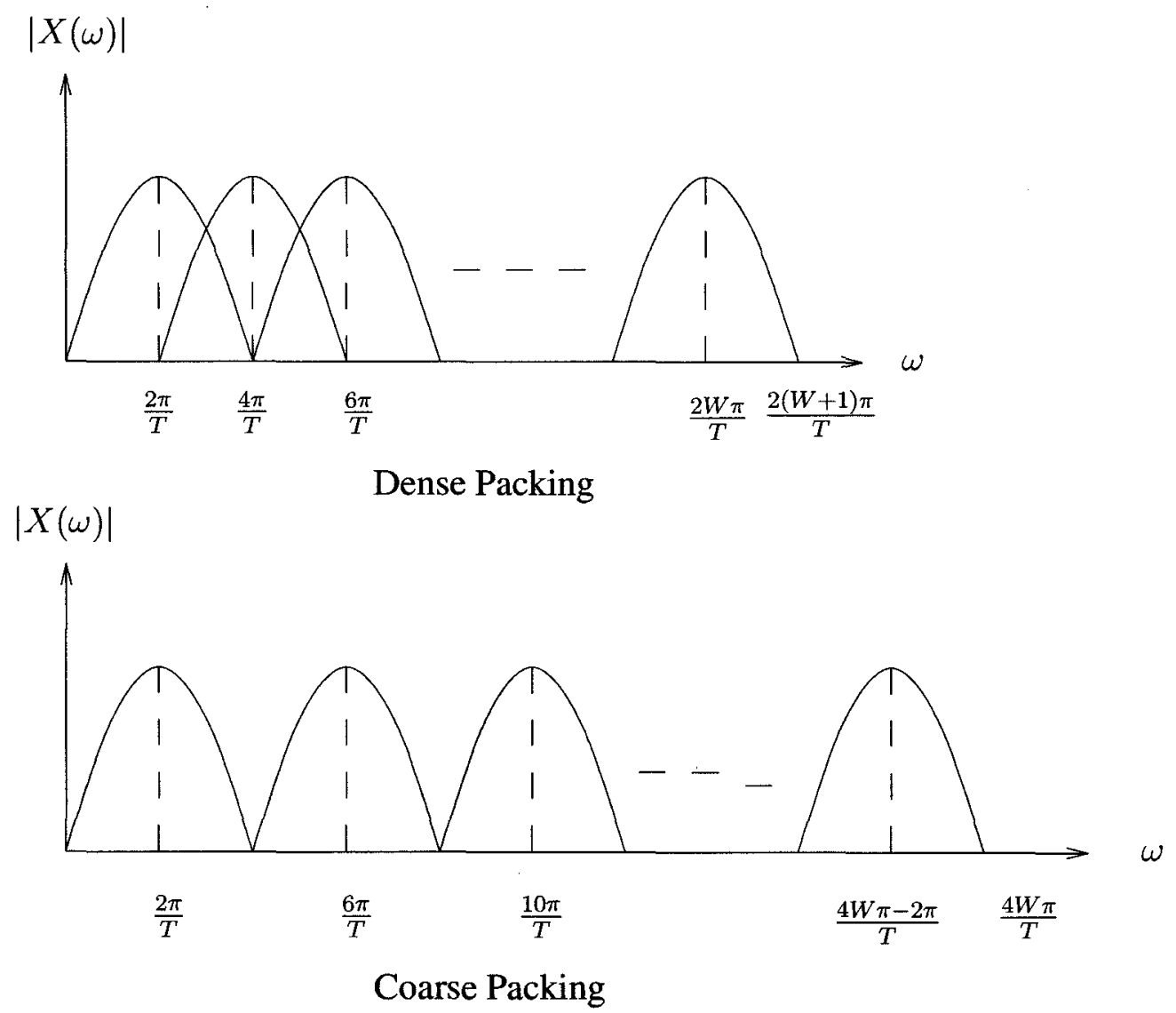

Figure 2.10: Dense packing and coarse packing of subcarriers 
In this thesis, we adopt the conventional term normal MSM system to denote an MSM wireless optical system with QPSK modulation employed on each carrier, rectangular pulse shape and symbol-by-symbol bias as is done in [31]. To facilitate the comparison of the average and peak optical power requirements of various techniques, define a reference on-off keying (OOK) wireless optical system with BER $10^{-6}$, rectangular pulse shape, average optical power $P_{\text {aook }}$ and peak optical power $P_{\text {pook }}$ as is conventionally done $[2,31]$. The unit-energy basis function for OOK is defined as [3]

$$
\phi_{\mathrm{OOK}}(t)=\frac{1}{\sqrt{T}} \operatorname{rect}\left(\frac{t}{T}\right)
$$

and the binary signals are

$$
\begin{aligned}
& S_{0}(t)=0, \quad 0 \leq t \leq T \\
& S_{1}(t)=2 P_{\text {aook }} \sqrt{T} \phi_{\mathrm{OOK}}(t)=2 P_{\mathrm{aook}} \operatorname{rect}\left(\frac{t}{T}\right), \quad 0 \leq t \leq T
\end{aligned}
$$

The constellation and basis function of OOK are shown in Figure 2.11. It can be seen in equations (2.32) and (2.33) that the average and peak optical powers of $S_{0}(t)$ are both zero, while the average and peak optical powers of $S_{1}(t)$ are $2 P_{\text {aook }}$. Therefore it is obvious that for equiprobable signalling, the average optical power of OOK system is $P_{\text {aook }}$ and the peak optical power is

$$
P_{\text {pook }}=2 P_{\text {aook }}
$$

Moreover, the probability of bit error for OOK system under an AWGN channel is

$$
P_{\text {eook }}=Q\left(\frac{P_{\text {aook }} \sqrt{T}}{\sqrt{\sigma^{2}}}\right)
$$

where $\sigma^{2}$ is the variance of the Gaussian noise and $Q()$ is the Gaussian tail function defined as

$$
Q(x)=\frac{1}{\sqrt{2 \pi}} \int_{x}^{+\infty} e^{-\frac{y^{2}}{2}} d y
$$




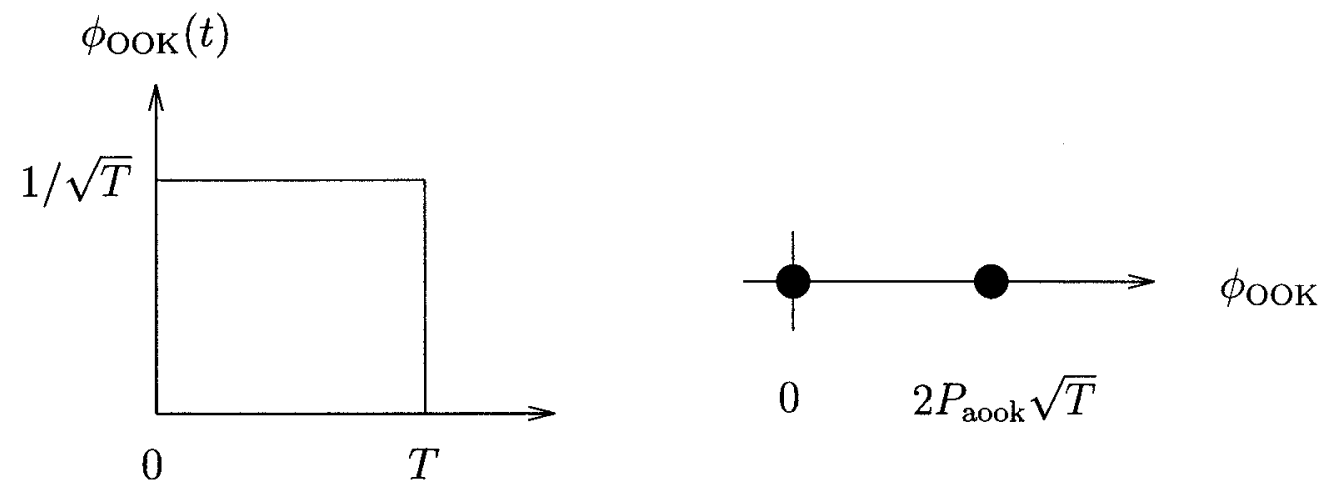

Figure 2.11: Constellation and basis function for OOK

Therefore by setting $P_{\text {eook }}=10^{-6}$ in equation (2.35), the numerical value of $P_{\text {aook }}$ is obtained. Then the value of $P_{\text {pook }}$ can be obtained using equation (2.34).

All wireless optical systems are designed to achieve the same bit rate and BER as the reference OOK system. The average optical power, $P_{a}$, and peak optical power, $P_{p}$, of various systems are compared versus the reference system using

$$
\begin{aligned}
& \rho=10 \log _{10} \frac{P_{a}}{P_{\text {aook }}}[\mathrm{dB}] \\
& \psi=10 \log _{10} \frac{P_{p}}{P_{\text {pook }}}[\mathrm{dB}]
\end{aligned}
$$

where $\rho$ is defined as the normalized average optical power and $\psi$ is defined as the normalized peak optical power. This metric allows the comparison of the average and peak optical powers for various MSM wireless optical systems and will be used throughout this thesis.

\subsection{Conclusions}

In this chapter, the channel model of MSM wireless optical systems is presented. The basic channel structure is discussed. The use of intensity modulation with direct detection enables low-cost optical transmitter and receiver devices such as LEDs and 
photodiodes. In diffuse configurations, high multipath distortion arises from multiple reflections of the signal and results in a low-pass channel frequency response. The major noise sources in wireless optical systems are the shot noise resulting from background light and the receiver preamplifier noise. The noise can be well modeled as being white, Gaussian and signal-independent. Low-frequency fluorescent light noise is also present in wireless optical links, however, its effect can be minimized by careful design of narrow-band carriers in MSM wireless optical systems.

Due to the intensity modulation technique used, a non-negativity constraint is placed on the amplitude of the electrical signal. Moreover, due to safety regulations and the limited dynamic range of the transmitter, the average and peak optical powers of wireless optical systems must also be limited. This in turn places a constraint on the average and peak amplitudes of the electrical signal. Furthermore, the modeling of MSM wireless optical systems is discussed. The architecture of the MSM transmitter and receiver are presented. The signal space and constellation of MSM signals are also discussed and the problem of average optical power reduction is defined.

Finally, a reference OOK wireless optical system and its average and peak optical powers are defined. A means to compare the average and peak optical power of various wireless optical systems is introduced. The models and definitions established in this chapter are used throughout this thesis. In Chapters 3 and 4, novel techniques for average optical power reduction in MSM wireless optical systems are proposed. Their average and peak optical powers are compared with those of the previous work based on the definitions in this chapter. 


\section{Chapter 3}

\section{In-Band Trellis Coding and DC Detection}

In Chapters 1 and 2 the problem of average optical power reduction for MSM wireless optical systems is defined and a means to compare the average and peak optical powers of various wireless optical systems is established. In this chapter, novel techniques are developed to address the problem of average optical power reduction in MSM wireless optical systems. Inspired by Ungerboeck's work on trellis codes [8] and Frenger's work on PCMS [65], we expand the signal set for the $W$ in-band carriers by adding a zero symbol to each subcarrier constellation. Trellis-coded modulation is applied over these in-band symbols using the expanded signal set, resulting in a reduction in the average optical power. By using this technique, a reduction in the peak optical power is also possible. We term this technique in-band trellis coding in this thesis. Additionally, a DC detection technique is proposed and the symbol-by-symbol bias is detected at the receiver to improve BER performance. The DC distance between MSM symbols can also be used to design trellis codes with better distance properties. As a result, DC detection technique achieves a reduction in the average optical power with a possible reduction in the peak optical power for MSM wireless optical systems. For simplicity, 
in this chapter we assume a flat low-pass channel frequency response over the entire in-band region and equal noise power at each carrier sub-channel. Therefore, no multipath distortion is observed. When multipath distortion is present, however, performance of this technique will be degraded and equalization techniques may be employed. It is assumed that all frequency carriers remain orthogonal at the output of the channel, and using a cyclic prefix in a FFT-based MSM system achieves this aim, as will be briefly discussed in Section 4.1.

This chapter starts in Section 3.1 with a brief review of the well-know technique of trellis-coded modulation [8]. The concept of constellation expansion, set partitioning, trellis structure and search for a mapping are discussed. The same heuristics are applied to maximize the Euclidean distance over sequences of MSM symbols in the design of trellis codes for MSM wireless optical systems.

According to the set partitioning scheme employed, two types of trellis-coded systems are proposed in this chapter. Trellis-coded MSM (TC-MSM) wireless optical systems are designed in Section 3.2 and each sub-constellation is partitioned to the level where there is only one constellation point. In Section 3.3, a different set partitioning scheme is introduced to group a larger number of MSM constellation points in a way to guarantee good distance properties. The resulting system is termed trellis-coded partitioned-MSM (TC-PMSM) wireless optical system.

The symbol-by-symbol bias used in MSM wireless optical systems is clearly correlated to the transmitted MSM symbol. In Section 3.5 this DC bias is detected at the receiver to improve BER performance. Moreover, the DC distance between MSM symbols is included in the design of trellis codes. In this manner, DC detection technique improves the average optical power efficiency of our systems and a reduction in the peak optical power is also possible. However, its performance may be degraded in the presence of low-frequency fluorescent light noise. 


\subsection{Trellis-coded Modulation}

Trellis-coded modulation, as the name suggests, combines coding and modulation at the transmitter. Traditional error-control coding techniques add redundancy to the transmitted symbols. They reduce transmission rate and trade bandwidth efficiency for error-control capabilities. Trellis-coded modulation, on the other hand, expands the signal set to realize redundancy in modulator output signals and does not reduce the bandwidth efficiency. The expanded signal set is partitioned into subsets such that the minimum Euclidean distance is increased. A convolutional code is then used to impose a structure on the sequence of output symbols. By combining the design of coding and signal mapping function, the Euclidean distances between the coded sequences of symbols are greatly increased. The decoder performs soft decision maximum likelihood sequence detection (MLSD) over the received sequence. The Viterbi algorithm is introduced in [71] for efficient implemention of the MLSD at the receiver.

The concept of trellis-coded modulation is best illustrated by an example given in [8]. An uncoded QPSK communication system in an AWGN channel is considered and its performance quantified. Based on this baseline system, the design of a trelliscoded system and its gain over the uncoded baseline system are illustrated.

\subsubsection{Baseline uncoded QPSK system}

Consider an uncoded QPSK communication system in an AWGN channel. Let $d_{\min }$ denote the minimum distance in the QPSK constellation as shown in Figure 3.1. It is assumed that each constellation point has unit energy. If signal $s_{1}$ is sent, the probability that a correct decision is made at the receiver is the area of region $Z_{1}$ and is given by 


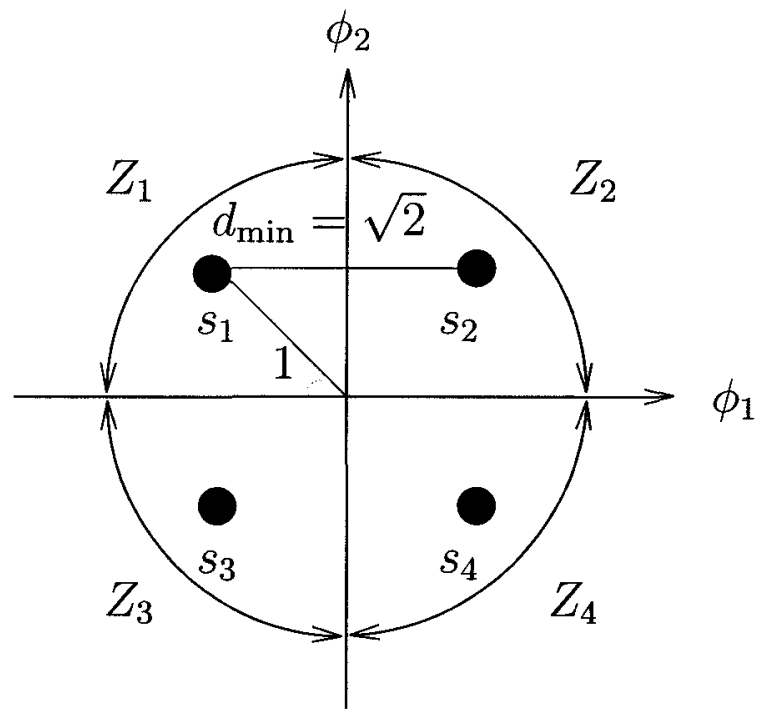

Figure 3.1: Unit-energy QPSK constellation

$$
P_{c}=\left[1-Q\left(\frac{d_{\min }}{2 \sigma}\right)\right]^{2}
$$

where $\sigma^{2}$ is the variance of the additive white Gaussian noise.

Assuming equiprobable signalling, the probability of error for uncoded QPSK system is given by

$$
P_{e}=1-P_{c}=1-\left[1-Q\left(\frac{d_{m i n}}{2 \sigma}\right)\right]^{2}=2 Q\left(\frac{d_{\min }}{2 \sigma}\right)-Q^{2}\left(\frac{d_{\min }}{2 \sigma}\right)
$$

The signal-to-noise ratio is defined as the ratio between the signal power and the noise power. In our QPSK system the Gaussian noise is two-dimensional, giving a noise power of $2 \sigma^{2}$. Therefore the SNR is defined as

$$
\mathrm{SNR} \triangleq\left(\frac{d_{\min }}{\sqrt{2}}\right)^{2} \cdot \frac{1}{2 \sigma^{2}}=\frac{d_{m i n}^{2}}{4 \sigma^{2}}
$$

Substituting (3.3) into (3.2), we get

$$
P_{e}=2 Q(\sqrt{\mathrm{SNR}})-Q^{2}(\sqrt{\mathrm{SNR}})
$$


To evaluate the performance of the uncoded QPSK system and trellis-coded systems, we compare the SNR required to get the same $P_{e}$ of $10^{-5}$. The value of $10^{-5}$ is chosen to be consistent with the analysis given in [8]. Setting $P_{e}=10^{-5}$ in (3.4) and solving it numerically, we get

$$
\mathrm{SNR}_{\mathrm{QPSK}}=12.9 \mathrm{~dB}
$$

Therefore a SNR of $12.9 \mathrm{~dB}$ is required for the uncoded QPSK system to achieve a BER of $10^{-5}$.

\subsubsection{Trellis-coded System}

To improve the performance of the uncoded QPSK system, the QPSK constellation is expanded to a 8-PSK constellation with the energy of each constellation point unchanged. This doubles the number of constellation points and increases the degree of freedom in choosing constellation points. This step is called constellation expansion. Note under the same assumption that each constellation point has unit energy, $d_{\min }$ is reduced to $\sqrt{2-\sqrt{2}}$ in 8-PSK constellation as opposed to $\sqrt{2}$ in QPSK constellation.

However, this expanded constellation will be partitioned in a way such that the minimum Euclidean distance is maximized at each partitioning step. This step is called set partitioning and is illustrated in Figure 3.2. The constellation points are grouped into disjoint sets defined as partitions such that the minimum Euclidean distances inside the partitions are maximized. From partitioning level 0 to level 1 , the points are divided into two partitions, both having a minimum Euclidean distance of $\sqrt{2}$. The value of $b^{0}$ determines which partition is selected. From partitioning level 1 to level 2, each partition is divided into two partitions, resulting in four partitions each with minimum Euclidean distance of 2 . Bits $b^{1}$ and $b^{0}$ together determine which partition is selected. At partitioning level 3, only one constellation point exist in each partition. Bits $b^{2}, b^{1}$ and $b^{0}$ together determines the point selected, which is the 

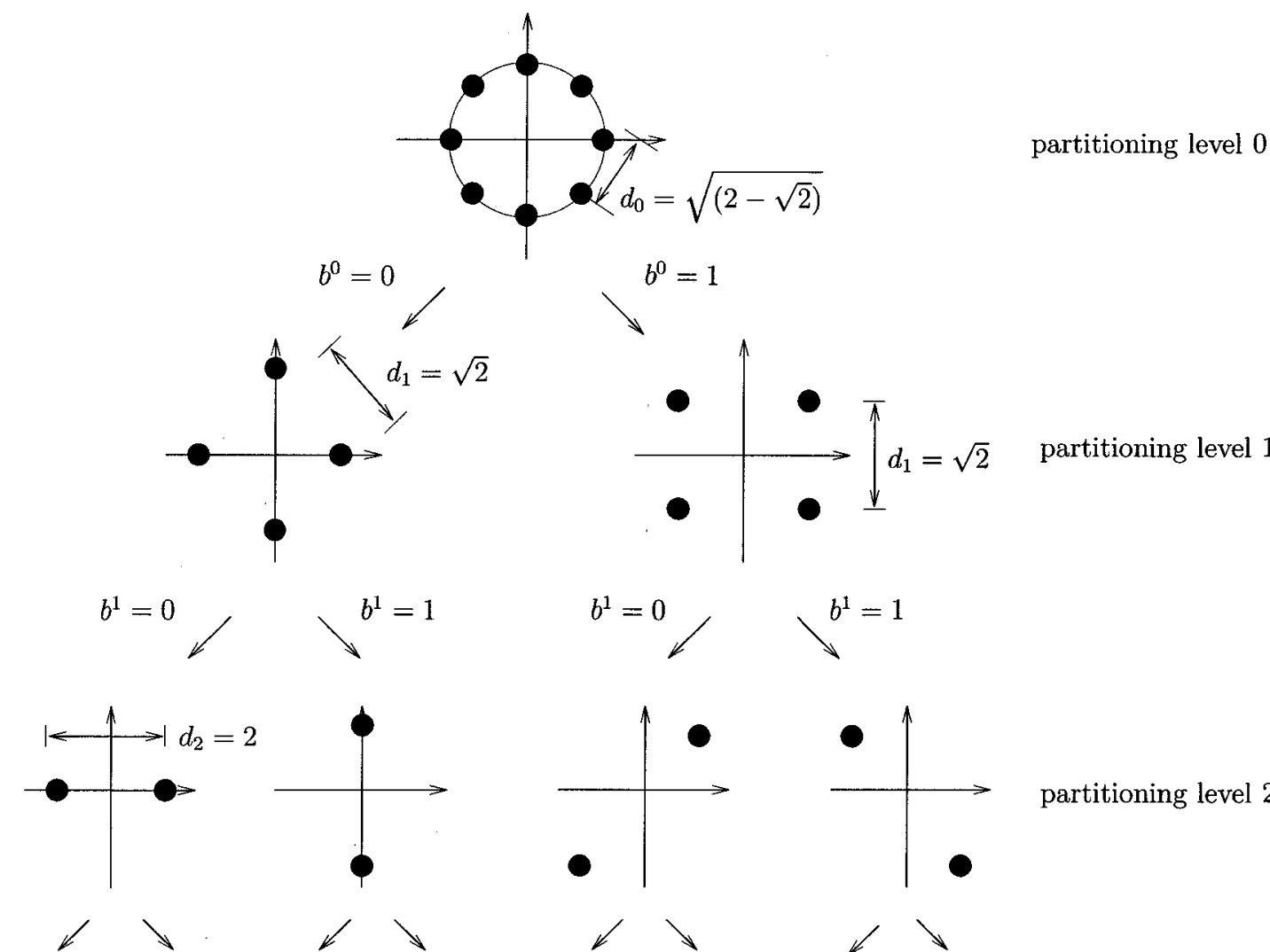

partitioning level 2
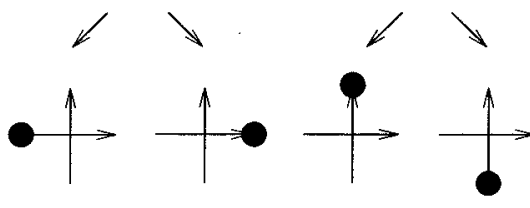

$b^{2}=0$

$b^{2}=1$

$b^{2}=0$

$b^{2}=1$
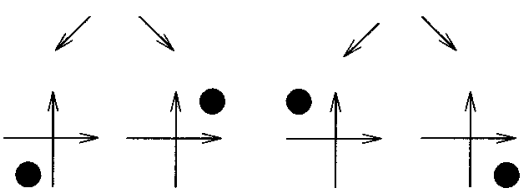

partitioning level 3

Figure 3.2: Set Partitioning of a 8-PSK constellation [8]

conventional three-bit representation of 8-PSK signals.

Given the expanded constellation and partitioning scheme, convolutional coding is then used to impose a structure on the sequence of output symbols. When the set partitioning is done to the level where there is only one constellation point, the error performance no longer depends on the minimum Euclidean distance between any individual constellation points. Instead, it depends on the minimum Euclidean distance between any two sequences of outputs. This will give an increased $d_{\min }$ for trellis-coded systems. In contrast, when more than one constellation point exist 


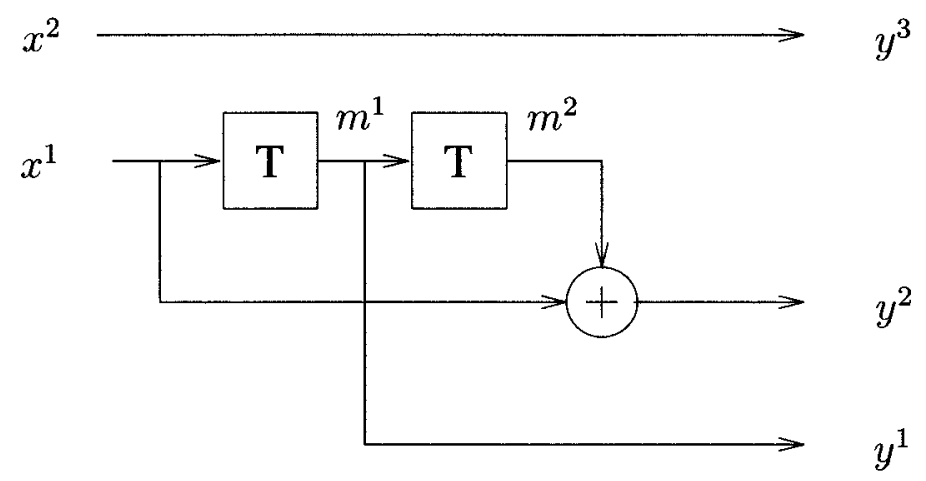

Figure 3.3: A 4-state convolutional encoder

in the partitions, the error performance at high SNR is determined by the smaller value between the minimum Euclidean distance inside the partition and the minimum Euclidean distance between any two sequences of outputs.

A convolutional code is an error-correction code which operates on the input bits and memory of the previous inputs to produce output bits. The memory of previous bits is realized using shift registers. The encoder structure of the 4-state code used in this example is shown in Figure 3.3. Two shift registers are connected in serial to input bit $x^{1}$, producing $m^{1}$, which is memory of $x^{1}$ at the previous time instance, and $m^{2}$, which is memory of $x^{1}$ two time instances before. The value of memory bits $\left(m^{1}, m^{2}\right)$ at a given time instance is called the state of the convolutional code at that time instance. The relationship between output bits $y^{1}, y^{2}$ and $y^{3}$ with inputs and memories is given by

$$
\begin{aligned}
& y^{1}=m^{1} \\
& y^{2}=x^{1}+m^{2} \\
& y^{3}=x^{2}
\end{aligned}
$$

where " + " is modulo-2 addition. 


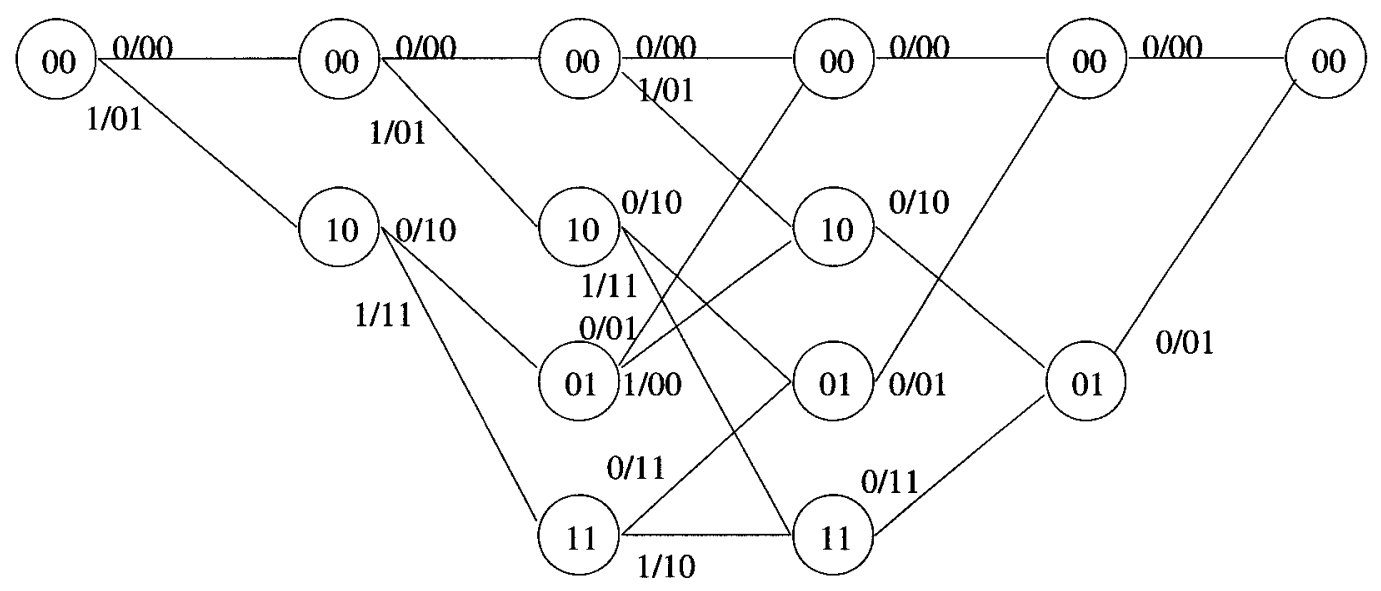

Figure 3.4: Trellis diagram for the 4-state convolutional code in Figure 3.3

A trellis is a useful diagram showing the state transitions and corresponding outputs of a convolutional code in time, depending on the inputs and current states. A section of a trellis diagram for the 4-state convolutional code in Figure 3.3 is drawn in Figure 3.4. Each node in the trellis corresponds to a distinct state at a given time. The value of the state at that time is written inside the node as $m^{1} m^{2}$. Each edge connecting two nodes represents a transition to a new state at the next instance of time. The digits written on each edge represents $x^{1} / y^{1} y^{2}$, where $x^{1}$ is current input and $y^{1}, y^{2}$ are outputs of the next time instance. Bits $y^{3}$ and $x^{2}$ are not shown here for simplicity. The trellis begins and ends at a specific known state. One important property of the trellis diagram is that to every possible input sequence there exist a unique path through the trellis, and vice versa. Each codeword sequence of the convolutional code corresponds to some unique path through the trellis.

The trellis-coded modulation transmitter is shown in Figure 3.5. Each possible combination of the output bits from the convolutional code is mapped into a constellation point, which refers to a certain output waveform to transmit in the channel. Thus, the remaining task is to find a good mapping between partitions of constellation points and trellis outputs such that the minimum Euclidean distance between 


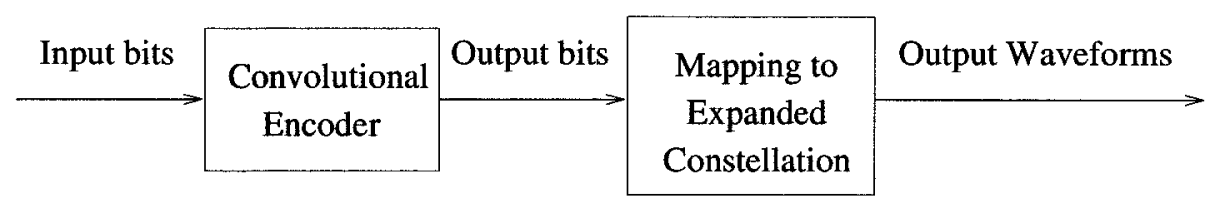

Figure 3.5: Trellis-coded modulation transmitter

any two sequences of outputs is maximized. In [8] the design is done either by hand or by an exhaustive computer search. For the 8-PSK constellation and 4-state convolutional code presented here, the optimal mapping which maximizes the minimum Euclidean distance is found by hand design and the minimum Euclidean distance is found to be 2, as opposed to $\sqrt{2}$ in uncoded QPSK system. Thus, by definition the SNR of trellis-coded system is doubled at the same noise power compared to uncoded systems. This predicts a $3 \mathrm{~dB}$ coding gain or equivalently a $3 \mathrm{~dB}$ savings in SNR to achieve the same BER theoretically. This has been verified by simulation: approximately $9.9 \mathrm{~dB}$ is required for trellis-coded 8-PSK system to achieve a $10^{-5} \mathrm{BER}$, as opposed to $12.9 \mathrm{~dB}$ for uncoded QPSK system shown earlier.

\subsection{Trellis-Coded MSM (TC-MSM)}

The block diagram of a trellis-coded MSM (TC-MSM) wireless optical transmitter is shown in Figure 3.6. The encoder block of a conventional MSM wireless optical system shown in Figure 2.6 is substituted by the trellis encoder and mapping block. Trellis codes are designed to impose a structure on the transmitted sequences of symbols. As in [8], the coded system design includes constellation expansion, set partitioning, choice of trellis structure and search of a mapping between constellation points and edges in the trellis. 


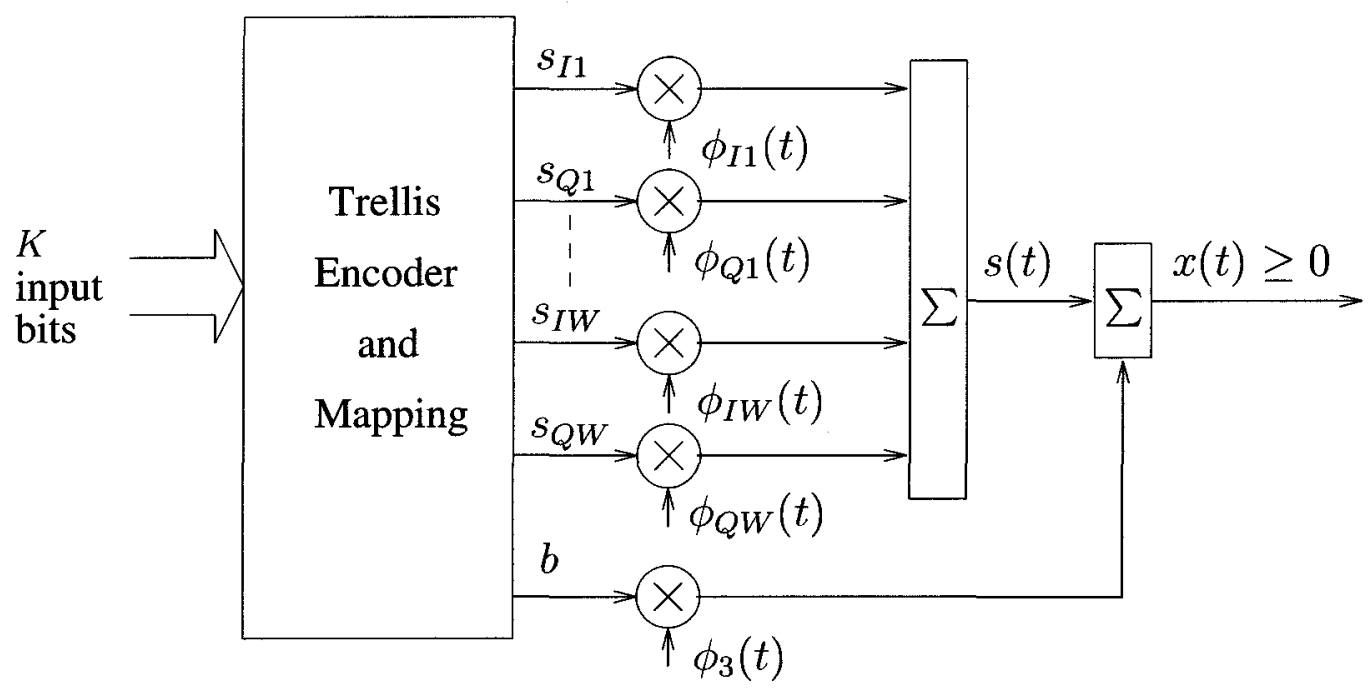

Figure 3.6: Trellis-coded MSM wireless optical transmitter

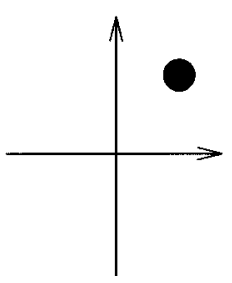

Carrier1

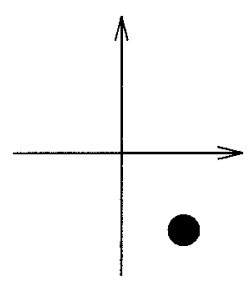

Carrier2

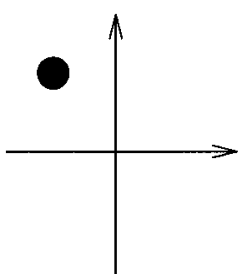

Carrier3

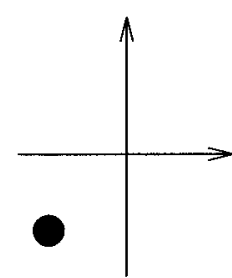

Carrier4

Figure 3.7: MSM constellation point consisting of 4 sub-constellations

\subsubsection{MSM constellation and expansion}

In an MSM wireless optical system with $W$ in-band carriers, define a sub-constellation as the constellation employed on each frequency carrier. Define an MSM constellation point as the collection of $W$ sub-constellations on each of the $W$ in-band subcarriers. An example of an MSM constellation point with $W=4$ is shown in Figure 3.7, in which all sub-constellation points are chosen from a QPSK constellation.

In a TC-MSM system, the constellation expansion is done by adding a zero amplitude to each sub-constellation as in [65]. This increase the degree of freedom in 


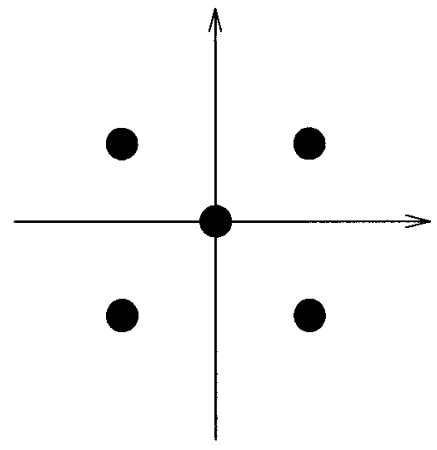

5-APSK

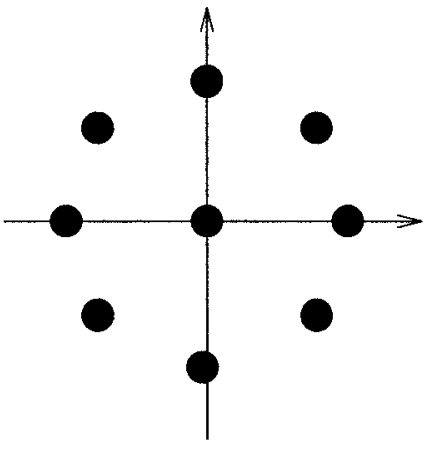

9-APSK

Figure 3.8: 5-APSK and 9-APSK sub-constellations

choosing constellation points. Later in Section 3.2.2 we will code over the increased degrees of freedom to achieve a reduction in the average optical power. Assuming either QPSK or 8-PSK is originally employed on each sub-constellation, the expanded constellations are called 5-APSK and 9-APSK constellations and are shown in Figure 3.8. Moreover, each sub-constellation is partitioned to the lowest level where only one constellation point is contained.

The expanded constellations can be constrained to have zero amplitude on at least $U$ subcarriers for all data symbols. Although this constraint limits the total number of available MSM constellation points, it can be shown later in Section 3.4 that it effectively reduces the average optical power requirement since less sinusoids are being added. Thus, for an $M$-state code using $W$ data carriers with $m$-APSK sub-constellation and constraint $U$, the total number of MSM constellation points is

$$
n_{s}=\sum_{i=U}^{W}\left(\begin{array}{c}
W \\
i
\end{array}\right) \times m^{W-i} .
$$




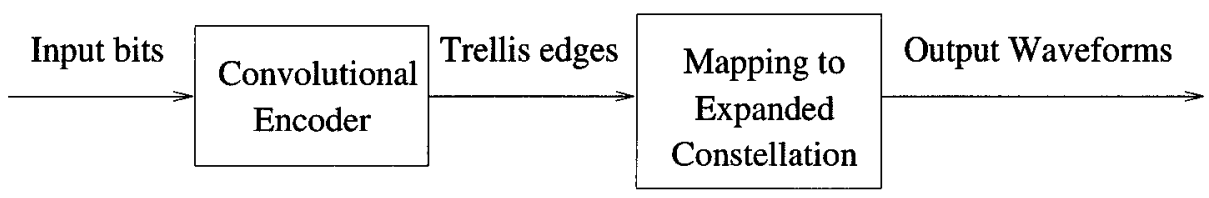

Figure 3.9: TC-MSM encoder and mapping block

\subsubsection{Code Design}

As discussed in Section 3.1, code development involves the design of a trellis structure and the mapping between MSM constellation points and output bits of the trellis code. In the TC-MSM system, however, the mapping is between MSM constellation points and trellis edges. In other words, the trellis code in TC-MSM system does not produce output bits. Each trellis edge, determined by both the input bits and the current state, is mapped to an MSM constellation point. The TC-MSM encoder and mapping block is shown in Figure 3.9, which is different from that of the conventional trellis-coded modulation.

The trellis structure is fixed to have $M$ states with $M$ branches leaving each state. Thus, at each stage of the trellis, $\log _{2} M$ bits could be sent by selecting the next state to go to from the current state. The MSM wireless optical system is targeted to send $K \geq \log _{2} M$ bits per symbol interval. Therefore, each state transition in the trellis must send $K-\log _{2} M$ bits using parallel transitions. An example of a two state code $(M=2)$ aiming to send $K=2$ bits per symbol period using $W=2$ in-band carriers with constraint $U=1$ is shown in Figure 3.10. The labels $S_{i}$ refer to MSM constellation points. Note $S_{1}$ and $S_{2}$ are two parallel edges from state 0 to state 0 , $S_{3}$ and $S_{4}$ are two parallel edges inside the branch from state 0 to state 1 , etc. At any stage of the trellis given the current state and two input bits $b^{0}$ and $b^{1}$, bit $b^{0}$ selects the next state, the other bit $b^{1}$ selects the parallel edge. This is illustrated in Table 3.1.

Given the trellis structure, the mapping between MSM constellation points and 

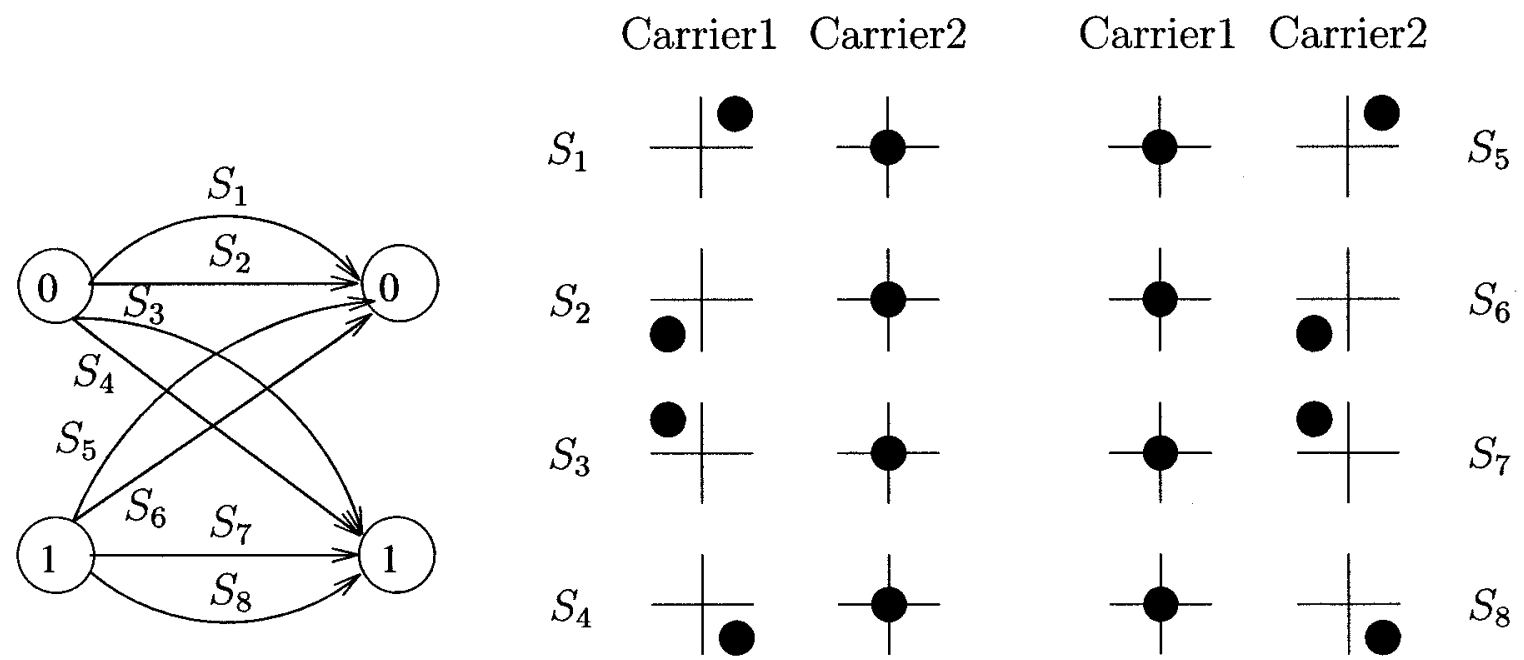

Figure 3.10: Trellis structure and MSM constellation for $K=2, M=2, W=2$ and $U=1$.

\begin{tabular}{|l|l|l|l|l|}
\hline Current state & $b^{0}$ & Next state & $b^{1}$ & Transmitted signal \\
\hline 0 & 0 & 0 & 0 & $S_{1}$ \\
\cline { 3 - 5 } & & & 1 & $S_{2}$ \\
\cline { 2 - 5 } & 1 & 1 & 0 & $S_{3}$ \\
\hline 1 & & & 1 & $S_{4}$ \\
\hline \multirow{2}{*}{0} & 0 & 0 & 0 & $S_{5}$ \\
\hline & & & 1 & $S_{6}$ \\
\cline { 3 - 5 } & 1 & 1 & 0 & $S_{7}$ \\
\hline & & & 1 & $S_{8}$ \\
\hline
\end{tabular}

Table 3.1: Outputs of trellis in Figure 3.10 given current state and inputs $b^{0}$ and $b^{1}$ 
edges in the trellis determines the free Euclidean distance, $d_{\text {free }}$, of the code. The $d_{\text {free }}$ asymptotically determines the code's error performance and is the minimum distance between any two paths in the trellis. Notice that since detection is done in the electrical domain, the goal of mapping design is to maximize the free Euclidean distance of the trellis code. In this scheme, the free distance can be limited by two MSM constellation points on the same branch or two different paths that start and end at the same state. Denote these distances as $d_{\mathrm{p}}$ and $d_{\mathrm{s}}$ respectively. Motivated by Ungerboeck's trellis code design heuristics [8], the following rules are applied when searching for a mapping:

1. Any MSM constellation point can occur only once at each stage of the trellis.

2. Pick parallel edges with maximum $d_{\mathrm{p}}$.

3. Pick points on edges leaving or entering the same state with maximum $d_{\mathbf{s}}$.

Rule 1 avoids confusion in decoding at each stage. For example, if a certain constellation point occurs more than once at each stage of the trellis, then in order to differentiate them at the receiver they must be located at edges originating from different states. In the code shown in Figure 3.10 and Table 3.1, at most two identical MSM constellation points can exist at each stage of the trellis, and one of them must be chosen from $S_{1}$ to $S_{4}$ while the other must be chosen from $S_{5}$ to $S_{8}$. However, even in this way, the correct choice between the identical points relies solely on whether the receiver knows the current state correctly. If the decoded state is wrong, then an incorrect decision is made. This can be prevented by prohibiting the use of identical constellation points at each stage of the trellis, hence rule 1 is applied in the design of TC-MSM codes. Rules 2 and 3 are designed to maximize $d_{\mathrm{p}}$ and $d_{\mathrm{s}}$ respectively.

The code with the largest $d_{\text {free }}$ should be obtained by implementing an exhaustive search over all possible mappings. However, by transmitting $K$ bits per symbol the 
number of MSM constellation points at each stage of the trellis is

$$
n_{\text {input }}=M \times 2^{K} .
$$

Notice $n_{\text {input }}$ is also the total number of different input data symbols used by the trellis code since rule 1 prohibits repeated use of identical MSM constellation points. Thus, the number of all possible mappings is

$$
\prod_{i=0}^{n_{\text {input }}-1}\left(n_{s}-i\right)
$$

where $n_{s}$ is given in equation (3.9). As a result, any exhaustive search is prohibitively expensive even for small $K$. A sub-optimal search algorithm is needed to find a good mapping between constellation points and trellis edges in a reasonable amount of time.

The search is greatly constrained if an MSM constellation point is selected at each search step. Suppose at the $f$ th step, $f-1$ MSM constellation points have been assigned. Let $d_{i}, i \in[1, f-1]$ denote the Euclidean distances between the $f$ th point and the $i$ th assigned point. Define $P_{f}$ as,

$$
P_{f}=\sum_{d=d_{1}}^{d_{f-1}} Q\left(\frac{d}{2 \sigma}\right) .
$$

The function $P_{f}$ is an upper bound on the probability that a wrong decision in decoding has been made regarding the $f$ th MSM constellation point over the $f-1$ assigned points. At each step, a point is chosen to minimize this upper bound. Thus, the following sub-optimal search algorithm for TC-MSM system is proposed.

Algorithm 1 Search for TC-MSM constellation point mapping.

1. Fix the first MSM constellation point.

2. For the $f$ th MSM constellation point $(f>1)$, select the point which minimizes $P_{f}$. 
3. If number of occurrences of current point $n>1$, return to step 2 and pick the point with the next smallest $P_{f}$.

4. Fix current point, add 1 to the number of occurrences of this point. If assignment not finished, go to step 2 .

End.

The search algorithm runs $n_{s}$ times to search all possible starting points in step 1. In the case of large $n_{s}, 100$ random starting points are selected. Note that the same search algorithm is implemented over both unconstrained MSM constellation $(U=0)$ and constrained MSM constellation $(U>0)$, despite the fact that constrained MSM constellation is a subset of unconstrained MSM constellation. In fact, the search over constrained MSM constellation often returned codes with smaller average optical power requirement than search results for the more general unconstrained MSM constellation, as will be presented in Section 3.4. This is due to the suboptimal search algorithm which improves the distance properties of the code stepwise rather than globally. Thus, there are two searches: constrained search $(U>0)$ and unconstrained search $(U=0)$. Additionally, for each search the following three sub-constellations types are used for each non-zero in-band carrier: QPSK, 5-APSK and 9-APSK. Since the optimal solution is not known and exhaustive search is too complex, it is not clear how far these suboptimal results are from the optimal solution.

In conclusion, for both constrained search and unconstrained search, the minimum average optical power code is chosen at a given bandwidth efficiency. For each code the non-zero in-band carriers have a certain sub-constellation type, although it may be QPSK, 5-APSK or 9-APSK. The performance of TC-MSM code found using both constrained and unconstrained search are presented in Section 3.4. 


\subsection{Trellis-Coded Partitioned-MSM (TC-PMSM)}

The TC-MSM systems work well with a small number of MSM constellation points. However, when a large number of constellation points is needed, i.e., for large $K$, the search algorithm 1 can consume a large amount of time and return codes with small $d_{\text {free. }}$. In this section, a trellis-coded partitioned-MSM (TC-PMSM) system is proposed to address this problem. A different set partitioning scheme is developed to group a large number of MSM constellation points. The search algorithm is modified to find codes with good distance property exploiting the set partitioning scheme.

Figure 3.11 illustrates the sub-constellation partitioning scheme for a 5-APSK constellation. Note that assuming all constellation points have unit energy except the zero-amplitude point, the minimum Euclidean distance is 1 for the unpartitioned constellation and $\sqrt{2}$ for the original QPSK constellation without the origin. Define the intra-set distance as the distance between constellation points located within the same partition. Similarly, define the inter-set distance as the distance between constellation points located within different partitions. At the first partitioning level, the minimum intra-set Euclidean distance for partitions $B 1$ and $B 2$ are both 2. Theoretically, doubling the minimum squared Euclidean distance gives a $3 \mathrm{~dB}$ electrical coding gain, which corresponds to a $1.5 \mathrm{~dB}$ optical gain. The design distance is fixed at 2 in this thesis since it surpasses gains in other average optical power reduction techniques for MSM wireless optical systems $[31,32]$ and can be achieved with moderate complexity.

Define a partition assignment (PA) to be the particular selection of the partitions over $W$ carriers. By definition, let $U$ carriers be assigned $B 0$ and the $W-U$ carriers assigned to $B 1$ or $B 2$. For a given $\mathrm{PA}, W-U$ bits are transmitted by selecting the assignment of partitions $B 1$ or $B 2$. Consider the example in Figure 3.12 with $W=5$, $U=2, M=2$ and $K=5$. The difference between this example and the previous 

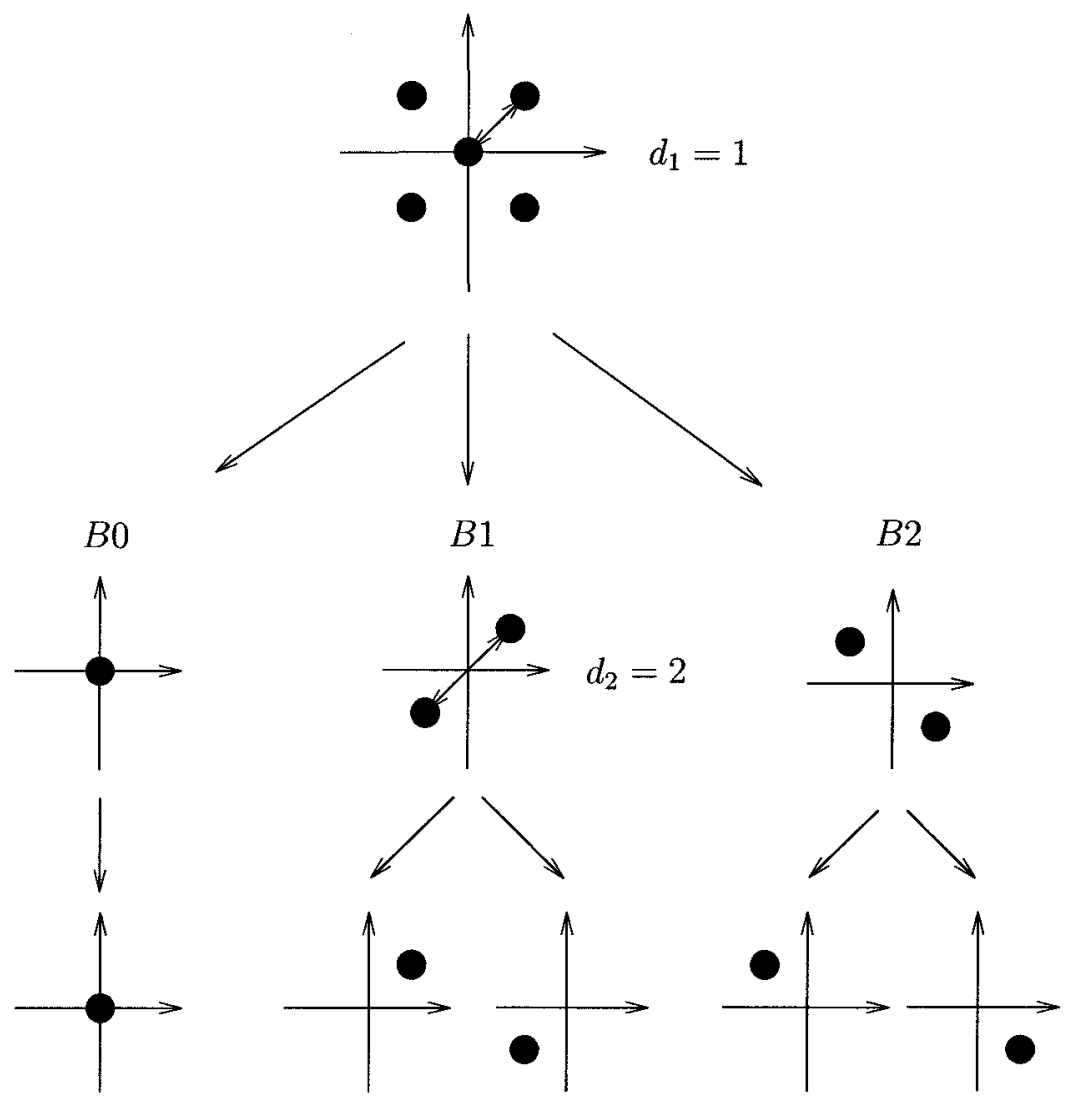

Figure 3.11: Partitioning of the 5-APSK sub-constellation. 


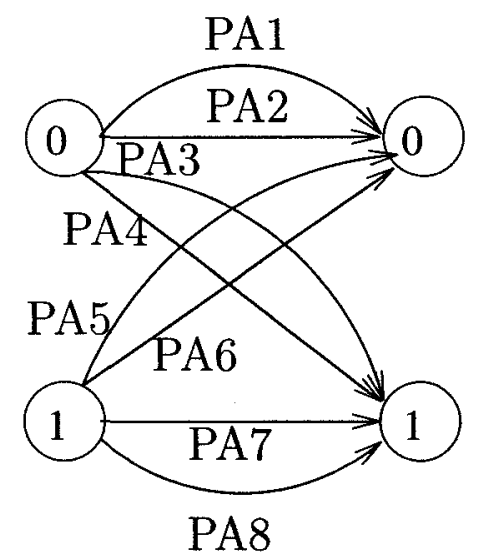

Figure 3.12: Trellis structure for $W=5, U=2, M=2$ and $K=5$.

example in Figure 3.10 is that a partition of constellation points is assigned to each edge instead of a single constellation point. One PA is transmitted on each edge which has two $B 0$ and three $B 1 / B 2$ assignments. The first bit $b^{0}$ selects the next state to go to from the current state, the second bit $b^{1}$ selects the parallel edge, thus determining the PA to transmit. The remaining three bits $b^{2}, b^{3}$ and $b^{4}$ determine the MSM constellation point in the selected PA to transmit. As in the design of TC-MSM systems, the mapping between input bits and output PAs and constellation points need to be designed to maximize the free Euclidean distance. This will be discussed shortly in this section. The output of edges (PAs) given the current state and inputs $b^{0}$ and $b^{1}$ is illustrated in Table 3.2. For example, given current state 0 and inputs $b^{0}=b^{1}=0$, it can be seen in Table 3.2 that PA1 is selected.

To explain the selection of constellation points inside a PA, consider an example where PA1 has partitions $(B 1, B 2, B 1, B 0, B 0)$ assigned to carrier 1 to carrier 5 respectively. Then the possible transmitted MSM constellation point given input bits $b^{2}, b^{3}$ and $b^{4}$ are shown in Figure 3.13.

Using this set partitioning scheme, $d_{\text {free }}$ can be limited by the MSM constellation points on parallel edges or on different paths that start and end at the same state. 


$$
b^{2}, b^{3}, b^{4}
$$

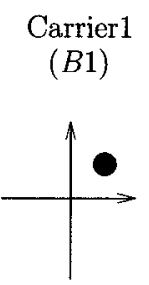

$$
\underset{(B 2)}{\text { Carrier2 }}
$$

000

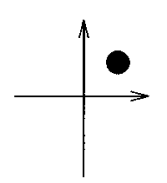

010
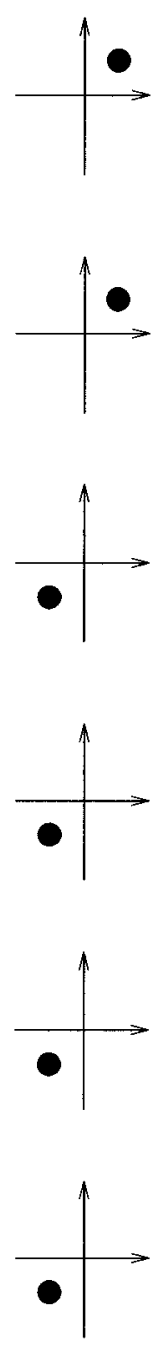

111
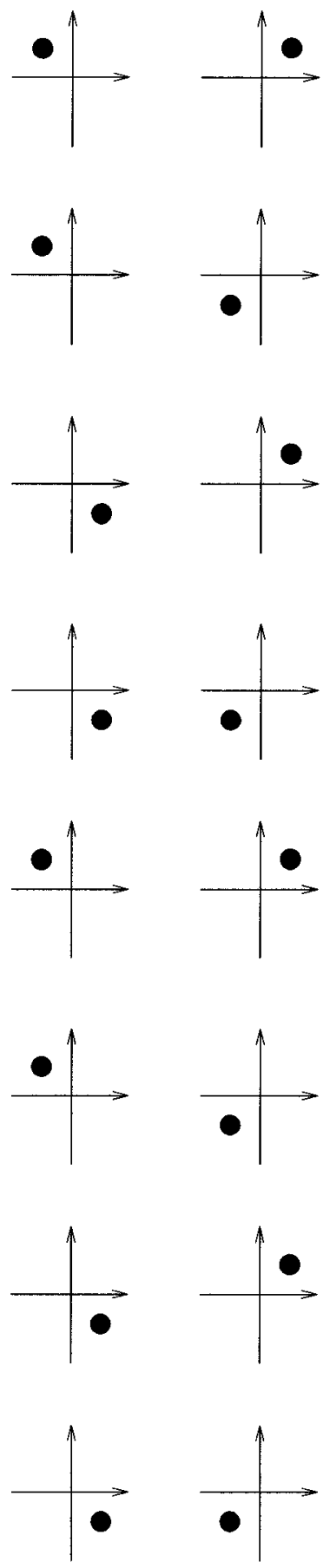

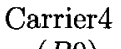

$(B 0)$

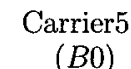

$(B 0)$
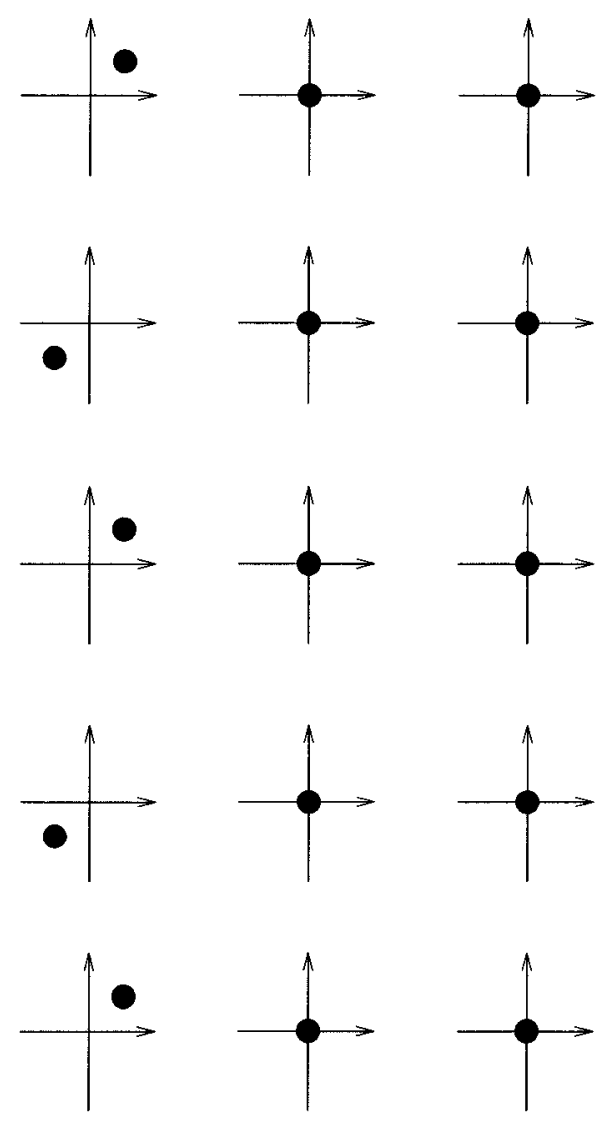
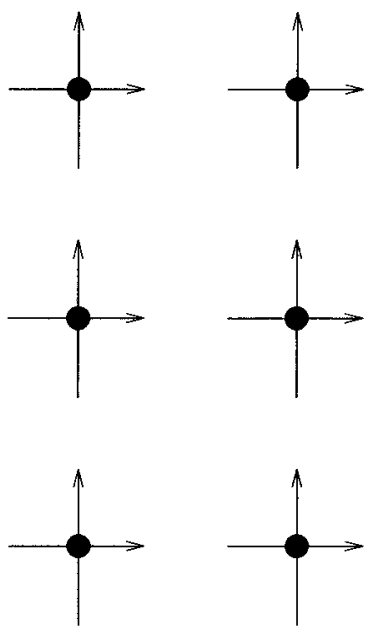

Figure 3.13: MSM constellation points chosen from PA1 given inputs $b^{2}, b^{3}$ and $b^{4}$ 


\begin{tabular}{|l|l|l|l|l|}
\hline Current state & $b^{0}$ & Next state & $b^{1}$ & Transmitted PA \\
\hline 0 & 0 & 0 & 0 & PA1 \\
\cline { 4 - 5 } & & & 1 & PA2 \\
\cline { 2 - 5 } & \multirow{2}{*}{1} & 1 & 0 & PA3 \\
\cline { 4 - 5 } & & & 1 & PA4 \\
\hline 1 & \multirow{2}{*}{0} & 0 & 0 & PA5 \\
\hline & & & 1 & PA6 \\
\cline { 2 - 5 } & \multirow{2}{*}{1} & 1 & 0 & PA7 \\
\cline { 3 - 5 } & & & 1 & PA8 \\
\hline
\end{tabular}

Table 3.2: PA Outputs of trellis in Figure 3.12 given current state and inputs $b^{0}$ and $b^{1}$

Denote these distances as $d_{\mathrm{p}}$ and $d_{\mathrm{s}}$ respectively. Unlike the TC-MSM system, $d_{\mathrm{p}}$ could be limited by either points inside the same PA or points in different PAs. Define $d_{\mathrm{PA}}$ as the minimum intra-PA distance and $d_{\mathrm{PA}-\mathrm{PA}}$ as the minimum inter-PA distance on the same edge. Note that the minimum $d_{\mathrm{PA}}$ is fixed to 2 due to our partitioning scheme. Note also that due to the symmetry of our trellis structure, the shortest path starting from one state and return to the same state requires 2 state transitions in the trellis. Therefore the minimum $d_{\mathrm{s}}$ arises due to a length 2 path. We denote the minimum distance between any two branches leaving or entering a state as $d_{\mathrm{smin}}$ and thus $d_{\mathrm{s}}^{2} \geq 2 d_{\mathrm{smin}}^{2}$. To ensure $d_{\text {free }} \geq 2$, we make $\sqrt{2 d_{\mathrm{smin}}^{2}} \geq 2$, and the following search procedure is proposed.

Algorithm 2 Search for TC-PMSM PA mapping.

1. Choose the first PA randomly.

2. Calculate the $d_{\mathrm{p}}$ and $d_{\mathrm{smin}}$ with every PA selected thus far. Pick the one with maximum $d_{\mathrm{p}}$. If $d_{\mathrm{p}}<2$ declare a search failure and stop algorithm. 
3. If $d_{\text {smin }}<\sqrt{2}$, go back to step 2 and pick the PA with next largest $d_{\mathrm{p}}$.

4. If number of occurrences of current PA $n>1$, return to step 2 and pick the PA with next largest $d_{\mathrm{p}}$.

5. Fix current PA, add 1 to the number of occurrences of this PA. If assignment not finished, go to step 2.

End.

The search is implemented using 5-APSK sub-constellation on each in-band carrier to limit the number of MSM constellation points and the search time at large $K$. The first PA is selected randomly from all the partition assignments. The performance of this system is shown in Section 3.4.

\subsection{Simulation Results}

Figures 3.14 and 3.15 plot the normalized average optical power $\rho$ defined in (2.37) and normalized peak optical power $\psi$ defined in (2.38) of the in-band trellis coding techniques presented in this chapter versus bandwidth efficiency $\eta$, defined in (2.30). For comparison, a "normal" MSM system defined in Chapter 2 and the minimumpower block coding technique in [31] are simulated for $W=1, \ldots, 7$. A recent SSPS code given in [34, Table II] is also simulated for comparison. Additionally, the tone injection method introduced in Chapter 1 for electrical PAPR reduction and included in VDSL standard [66] is applied directly to an MSM wireless optical system. One tone is modified and the best choice is found using an exhaustive search. All the systems are designed to achieve the same bit rate and the same BER of $10^{-6}$. The analytical models presented in Section 2.6 are used to simulate a working system. An explanation of the legends used in figures within this chapter is given in Table 3.3.

Applying the algorithms in Section 3.2.2 and Section 3.3, both TC-MSM codes and TC-PMSM codes are designed using different choices of $W, U, M$ and $K$ at the 


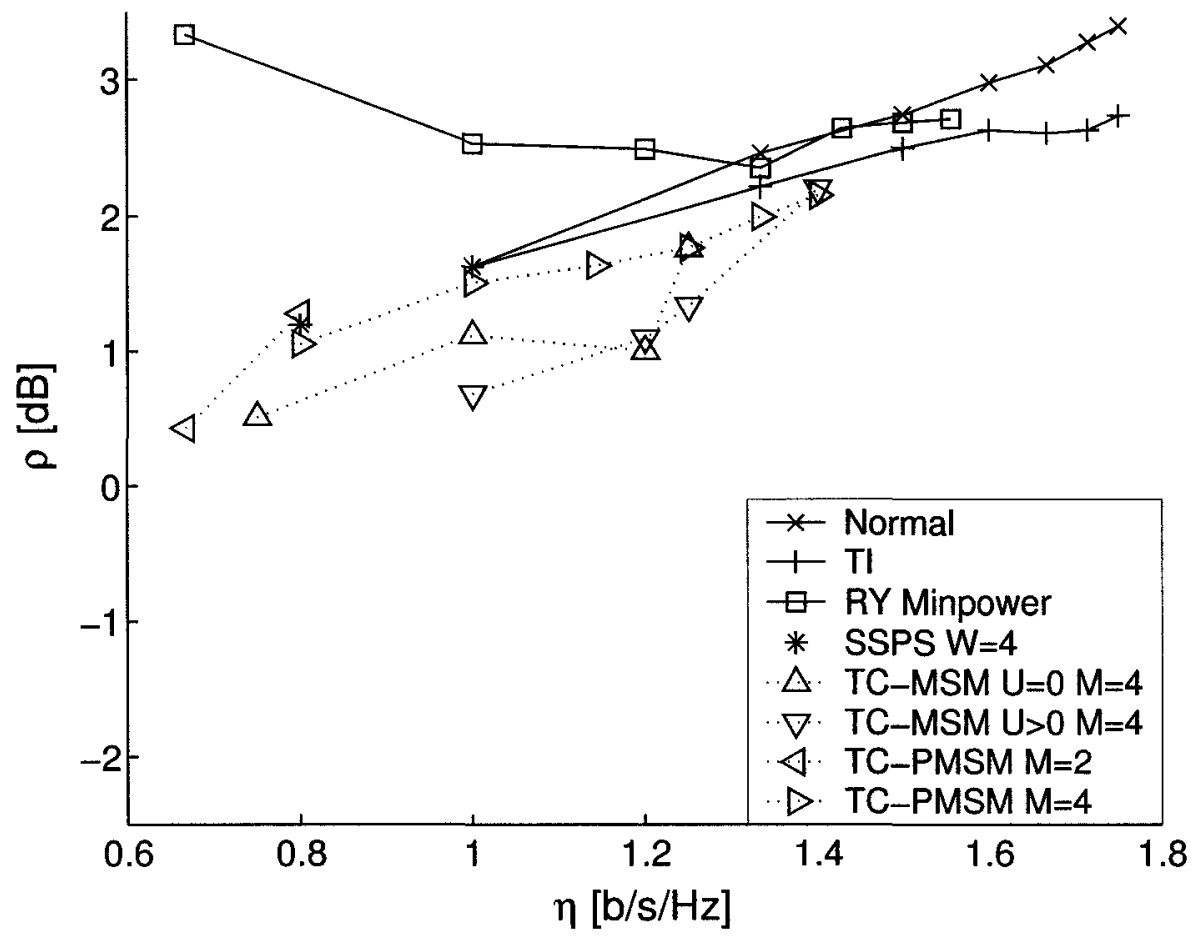

Figure 3.14: Normalized average optical power $(\rho)$ versus bandwidth efficiency $(\eta)$ for TC-MSM and TC-PMSM

\begin{tabular}{|l|l|}
\hline Normal & "Normal" MSM system (Section 2.7) \\
\hline TI & Tone injection method [66] (Section 1.3) \\
\hline RY Minpower & You's Minimum block coding technique [31] (Section 1.4) \\
\hline SSPS & SSPS technique [34] (Section 1.4) \\
\hline TC-MSM & Trellis-coded MSM (Section 3.2) \\
\hline TC-PMSM & Trellis-coded partitioned-MSM (Section 3.3) \\
\hline TCMSM Best & $\begin{array}{l}\text { Best (minimum average optical power) } \\
\text { in-band trellis codes (Section 3.4) }\end{array}$ \\
\hline DC & DC detection technique (Section 3.5) \\
\hline
\end{tabular}

Table 3.3: Explanation of legends used in performance figures in Chapter 3 


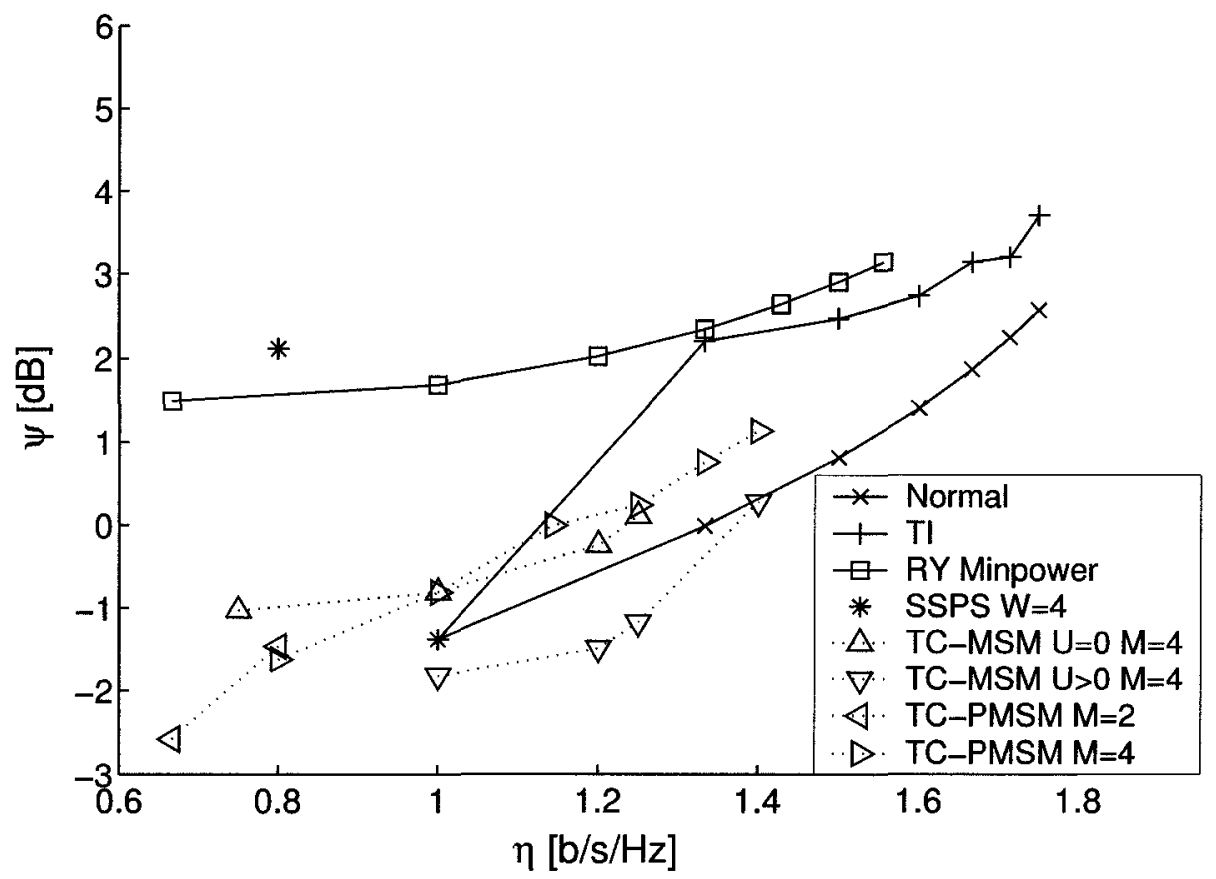

Figure 3.15: Normalized peak optical power $(\psi)$ versus bandwidth efficiency $(\eta)$ for TC-MSM and TC-PMSM 
same bit rate and the same BER of $10^{-6}$. For TC-MSM systems, the codes found using both constrained search $(U>0)$ and unconstrained search $(U=0)$ are shown. For each search with a specific sub-constellation type at a given $N, U, K$, and $M$, the search algorithm runs $n_{s}$ times to search all possible starting points in step 1 . In the case of large $n_{s}, 100$ random starting points are selected. After searching using all three sub-constellation types, the search terminates with a set of codes having some maximum $d_{\text {free }}$ and smallest number of $d_{\text {free }}$ paths. In the case where there is more than one such code, the average optical power of each of the codes are then compared. The code with minimum average optical power at each bandwidth efficiency is chosen. The properties of each codes are described in Table 3.4.

In Figure 3.14 it is evident that bandwidth efficiency is traded for average optical power efficiency for all power reduction schemes, as is conventional in optical signalling design [72]. The proposed in-band trellis coding techniques outperform existing techniques at the lower bandwidth efficiency region. At higher bandwidth efficiencies, the free Euclidean distance of the codes is smaller due to the reduced degrees of freedom in the code design. As a result the average optical power gain is reduced. The TC-MSM systems with $U=0$ offers a reduction of $0.51 \mathrm{~dB}$ in average optical power while increasing the peak optical power by at most $0.57 \mathrm{~dB}$ compared at the same bandwidth efficiency. In contrast, the constrained TC-MSM systems with $U>0$ achieved a greater reduction of up to $0.95 \mathrm{~dB}$ in average optical power while simultaneously reducing the peak optical power by $0.44 \mathrm{~dB}$. The reductions are explained by the increased free distance of the trellis codes, which in turn reduces the amplitude required at the transmitter to achieve a $10^{-6}$ BER. It is seen in Table 3.4 that the average optical power performance of the codes mainly depends on its $d_{\text {free }}$.

Note that the peak optical power of in-band trellis codes are not always reduced comparing to the normal MSM systems, as shown in Figure 3.15. This is because the average optical power is the only criterion in the selection of codes at each bandwidth 


\begin{tabular}{|c|c|c|c|c|c|c|c|c|c|}
\hline & $\eta$ & $W$ & $K$ & $U$ & $M$ & Sub-constellation & $d_{\text {free }}$ & $\rho$ & $\psi$ \\
\hline \multirow{4}{*}{$\begin{array}{l}\text { TC-MSM } \\
\text { Constrained } \\
\text { Search }\end{array}$} & 1.00 & 4 & 5 & 2 & 4 & 9-APSK & 4 & 0.68 & -1.82 \\
\hline & 1.20 & 4 & 6 & 2 & 4 & 9-APSK & 3.17 & 1.10 & -1.49 \\
\hline & 1.25 & 3 & 5 & 1 & 4 & 9-APSK & 3.17 & 1.34 & -1.18 \\
\hline & 1.40 & 4 & 7 & 1 & 4 & 9-APSK & 1.75 & 2.20 & 0.27 \\
\hline \multirow{4}{*}{$\begin{array}{l}\text { TC-MSM } \\
\text { Unconstrained } \\
\text { Search }\end{array}$} & 0.75 & 3 & 3 & 0 & 4 & 9-APSK & 12 & 0.52 & -1.03 \\
\hline & 1.00 & 3 & 4 & 0 & 4 & QPSK & 6 & 1.12 & -0.81 \\
\hline & 1.20 & 4 & 6 & 0 & 4 & 9-APSK & 8 & 1.00 & -0.24 \\
\hline & 1.25 & 3 & 5 & 0 & 4 & 9-APSK & 2.59 & 1.93 & 0.27 \\
\hline \multirow[t]{8}{*}{ TC-PMSM } & 0.67 & 2 & 2 & 1 & 2 & 5-APSK & 2 & 0.43 & -2.58 \\
\hline & 0.80 & 4 & 4 & 2 & 2 & 5-APSK & 2 & 1.28 & -1.46 \\
\hline & 0.80 & 4 & 4 & 2 & 4 & 5-APSK & 2 & 1.05 & -1.62 \\
\hline & 1.00 & 5 & 6 & 2 & 4 & 5-APSK & 2 & 1.51 & -0.81 \\
\hline & 1.14 & 6 & 8 & 2 & 4 & 5-APSK & 2 & 1.64 & 0.00 \\
\hline & 1.25 & 7 & 10 & 2 & 4 & 5-APSK & 2 & 1.77 & 0.24 \\
\hline & 1.33 & 8 & 12 & 2 & 4 & 5-APSK & 2 & 2.00 & 0.75 \\
\hline & 1.40 & 9 & 14 & 2 & 4 & 5-APSK & 2 & 2.15 & 1.13 \\
\hline
\end{tabular}

Table 3.4: Properties of each TC-MSM and TC-PMSM codes (assuming $d_{0}^{2}=1$ ) 
efficiency. In general, the peak optical power of in-band trellis-coded systems can be viewed as not heavily affected comparing to normal MSM systems. Moreover, note that in-band trellis codes operates well at lower bandwidth efficiencies. At higher bandwidth efficiencies, the tone injection method achieved good average optical power reductions up to $0.66 \mathrm{~dB}$ with a significant increase of peak optical power up to $2.22 \mathrm{~dB}$. However, in Chapter 4 another technique will be proposed and shown to outperform existing techniques at higher bandwidth efficiencies.

It should be noted that the constraint $U$ on the number of zero-amplitude carriers for all MSM constellation points also affects the average optical power efficiency of the code. For example, in Table 3.4 although the constrained TC-MSM code at $\eta=1$ has a smaller $d_{\text {free }}$ than the unconstrained TC-MSM code at $\eta=1.2$, it has a lower average optical power requirement due to the presence of $U=2$ zero-amplitude carriers, which helps reduce both average and peak optical powers by limiting the number of sinusoids in the MSM waveform.

As shown in Figures 3.14 and 3.15, the performance of TC-PMSM systems are slightly worse than those of TC-MSM systems at most times, although a greater reduction in peak optical power can be seen at low bandwidth efficiency. This can be explained by the fact that TC-PMSM codes have a lower $d_{\text {free }}$ than TC-MSM codes due to the set partitioning scheme employed, which limits $d_{\text {free }}$ to 2 . This confirms that $d_{\text {free }}$ asymptotically determines a trellis code's performance. Furthermore, it should be noted that while TC-PMSM supports a larger $K$ value due to the use of our set partitioning scheme, the number of carriers employed $W$ is also large. This restricts the bandwidth efficiency of TC-PMSM codes and make their average optical power efficiency low when comparing at the same bandwidth efficiency.

In conclusion, given the performance of all in-band trellis coding techniques, we 


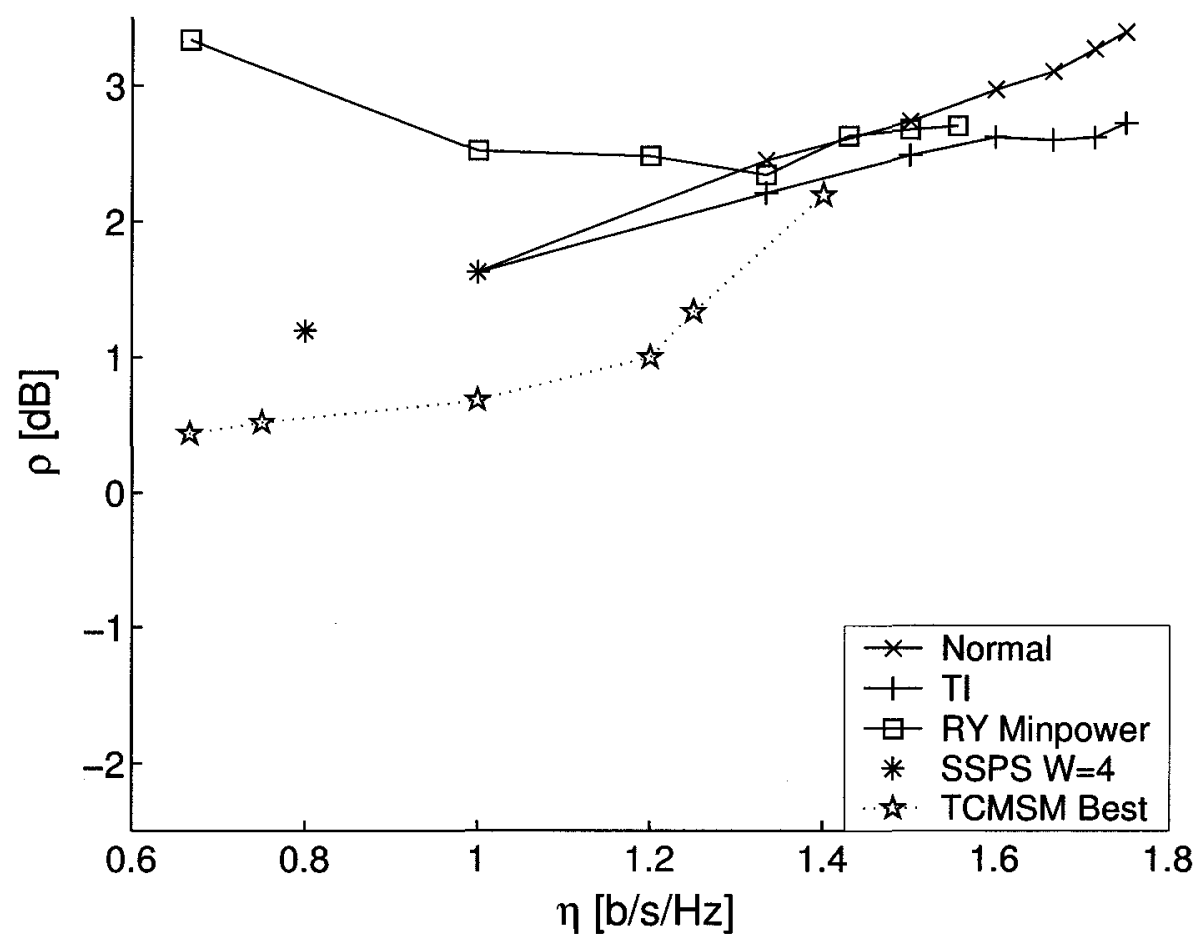

Figure 3.16: Normalized average optical power $(\rho)$ versus bandwidth efficiency $(\eta)$ for "best" (minimum average optical power) in-band trellis codes

now pick the best in-band trellis codes with minimum average optical power requirement at each bandwidth efficiency. The resulting performance are shown in Figures 3.16 and 3.17 and Table 3.5 as a summary of the performance of in-band trellis coding techniques.

Note that conventionally, $\rho$ is plotted against $W$ or $K$ [31-34]. Such plots are shown in Figures 3.18 and 3.19. The constrained TC-MSM systems with $W=3$, $U>0$ at $\eta=0.75$ achieved an average optical power reduction of $2.22 \mathrm{~dB}$ optical over normal MSM systems with a simultaneous peak power reduction of $1.84 \mathrm{~dB}$ optical. Another constrained TC-MSM systems with $W=4, U>0$ at $\eta=1$ achieved an average optical power reduction of $2.29 \mathrm{~dB}$ optical over normal MSM systems with a simultaneous peak power reduction of $3.23 \mathrm{~dB}$ optical. However, the plot of $\rho$ and 


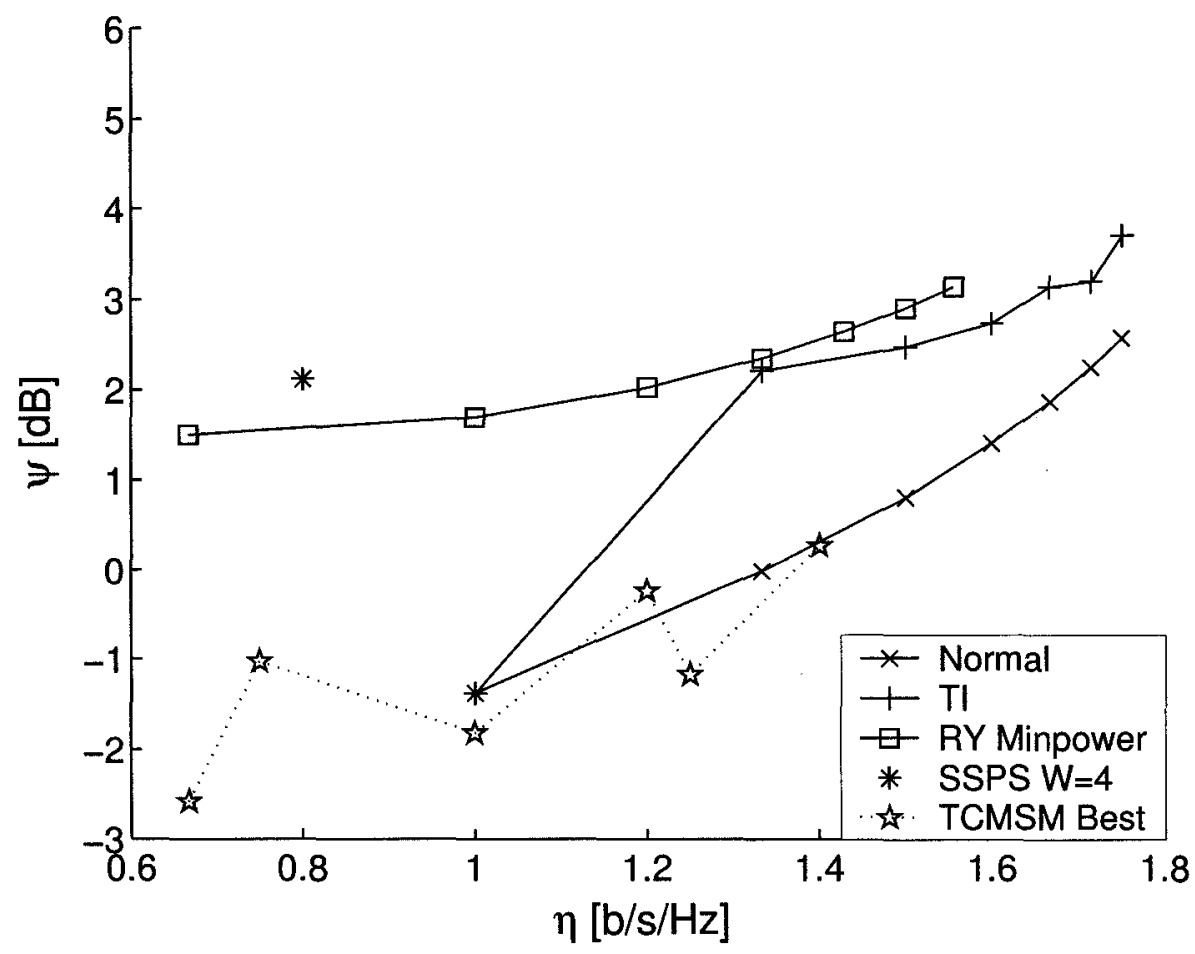

Figure 3.17: Normalized peak optical power $(\psi)$ versus bandwidth efficiency $(\eta)$ for "best" (minimum average optical power) in-band trellis codes

\begin{tabular}{|l|l|l|l|l|l|l|l|l|l|}
\hline Scheme & $\eta$ & $W$ & $K$ & $U$ & $M$ & Sub-constellation & $d_{\text {free }}$ & $\rho$ & $\psi$ \\
\hline TC-PMSM & 0.67 & 2 & 2 & 1 & 2 & 5-APSK & 2 & 0.43 & -2.58 \\
\hline TC-MSM & 0.75 & 3 & 3 & 0 & 4 & 9-APSK & 12 & 0.52 & -1.03 \\
\hline TC-MSM & 1.00 & 4 & 5 & 2 & 4 & 9-APSK & 4 & 0.68 & -1.82 \\
\hline TC-MSM & 1.20 & 4 & 6 & 0 & 4 & 9-APSK & 8 & 1.00 & -0.24 \\
\hline TC-MSM & 1.25 & 3 & 5 & 1 & 4 & 9-APSK & 3.17 & 1.34 & -1.18 \\
\hline TC-MSM & 1.40 & 4 & 7 & 1 & 4 & 9-APSK & 1.75 & 2.20 & 0.27 \\
\hline
\end{tabular}

Table 3.5: Properties of the "best" (minimum average optical power) in-band trellis codes (assuming $d_{0}^{2}=1$ ) 


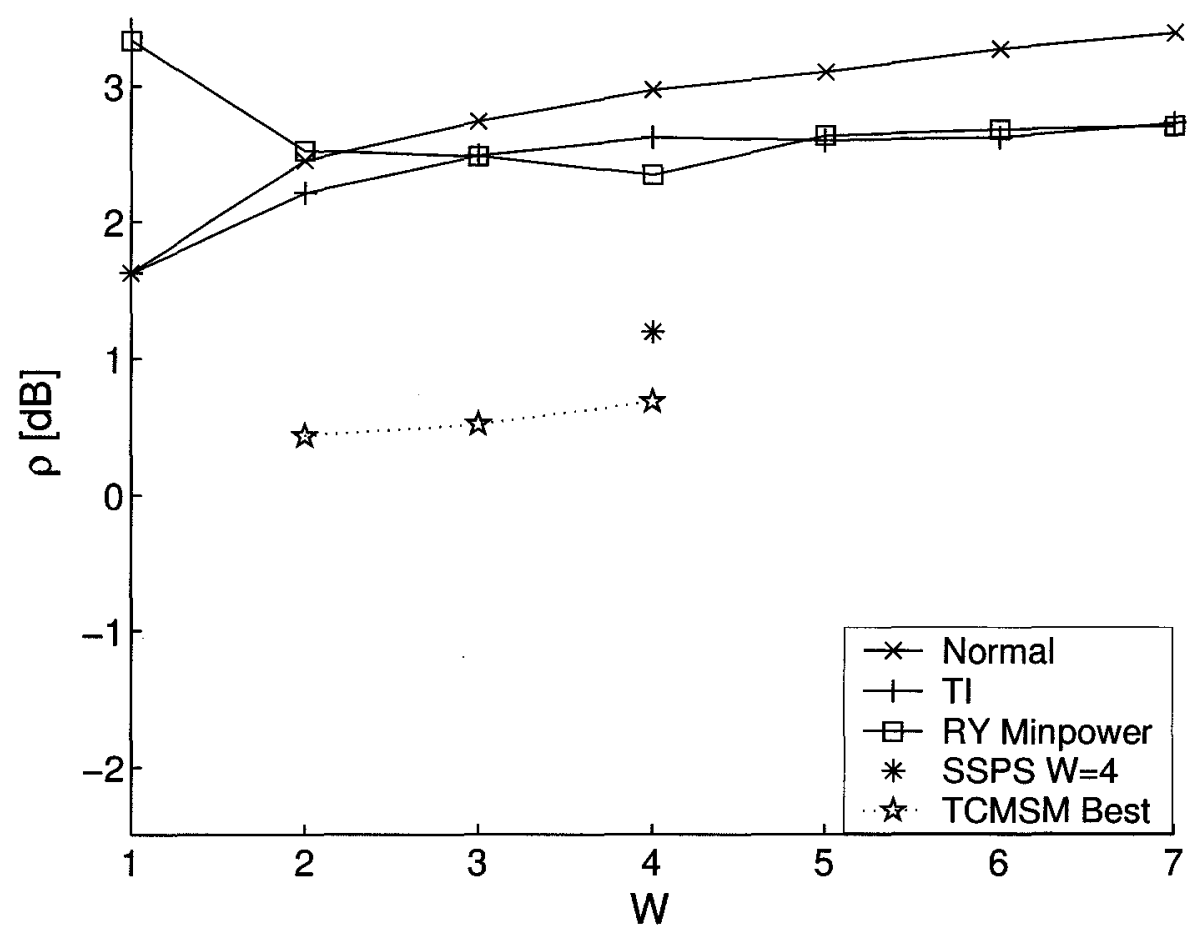

Figure 3.18: Normalized average optical power $(\rho)$ versus number of in-band carriers $(W)$ for best in-band trellis codes

$\psi$ versus $\eta$ more clearly justifies the performance of various schemes versus their cost and is therefore a better comparison.

Since reduction in optical power is equivalent to twice that value in electrical power, in-band trellis coding technique achieved a significant amount of reduction in both average and peak optical powers. The costs, however, are the increased transmitter and receiver complexity due to use of trellis encoder and Viterbi decoder. Thus, in-band trellis coding techniques are suitable for applications where both average and peak optical powers are tightly constrained, and the increase in complexity can be tolerated. 


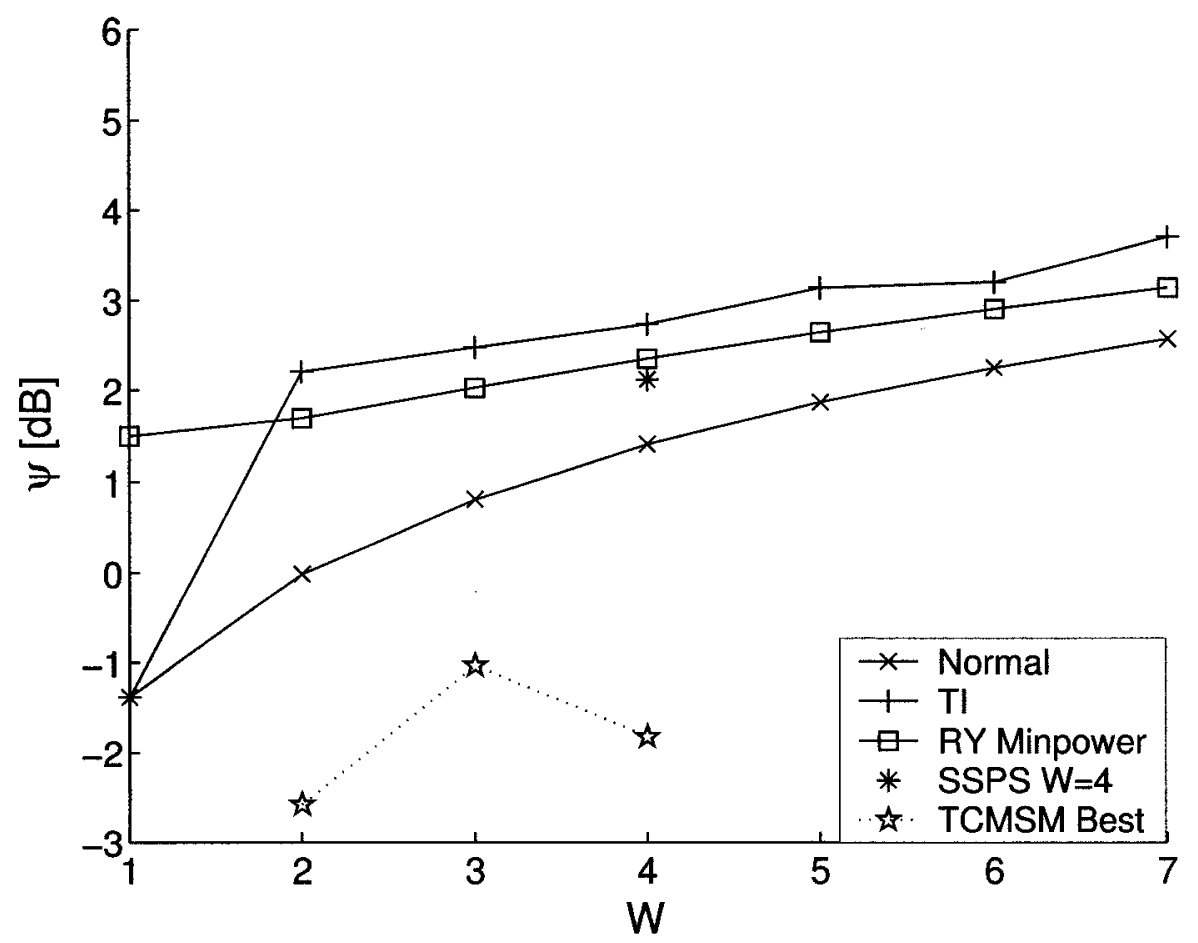

Figure 3.19: Normalized peak optical power $(\psi)$ versus number of in-band carriers $(W)$ for best in-band trellis codes 
MSM Symbols

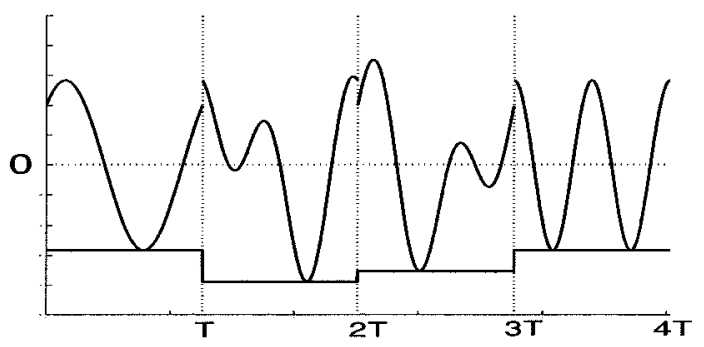

Required Bias

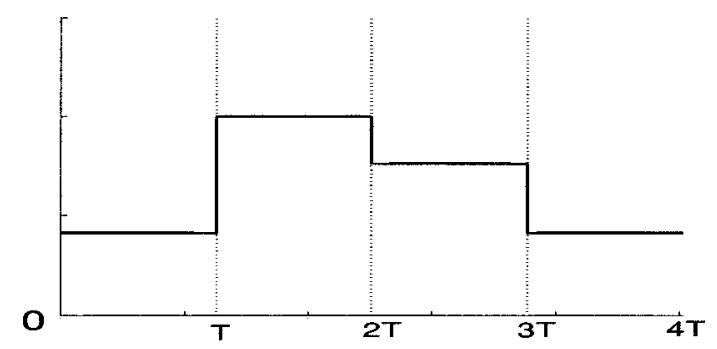

Figure 3.20: Four MSM symbols and the resulting symbol-by-symbol bias signal

\subsection{Level Detection}

At the receiver, the received signal $y(t)$ is match filtered to $\phi_{I i}(t)$ and $\phi_{Q i}(t)$ as described in Chapter 2 to extract sufficient statistics for detection. However, the symbol-by-symbol bias is clearly correlated to the transmitted signal and provides additional information. This is illustrated by Figure 3.20, where four MSM symbols are the resulting symbol-by-symbol bias signal are shown. In this section, this correlated information is exploited to provide a signal space diversity [67], which improves detection of the transmitted symbol. It is assumed that the noise powers at each carrier subchannel including the DC bin are the same.

In Section 2.6, the unit-energy basis function for the symbol-by-symbol bias is defined in (2.17) and is repeated here for convenience,

$$
\phi_{3}(t)=\frac{1}{\sqrt{T}} \operatorname{rect}\left(\frac{t}{T}\right) .
$$

The projection of the transmitted signal at the $j$ th symbol period onto the basis 
function is also defined in (2.20) and repeated here,

$$
b^{(j)}=\int_{j T}^{(j+1) T} x(t) \phi_{3}(t-j T) d t .
$$

At the receiver, we obtain an estimate $\hat{b}^{(j)}$ of $b^{(j)}$ by projecting the received signal $y(t)$ onto the $\phi_{3}$ dimension

$$
\hat{b}^{(j)}=\int_{j T}^{(j+1) T} y(t) \phi_{3}(t-j T) d t
$$

Thus the DC level $\hat{b}^{(j)}$ provides a degree of diversity and is used at the receiver to improve detection. As a result, independent detection over each carrier, which is used in normal MSM systems, is not possible by applying DC detection at the receiver. Joint detection over all carriers and the DC bin is done at the receiver using an MLSD as before. Moreover, the DC level increases the distance between MSM constellation points. In fact, such diversity can be used in the search for a mapping between MSM constellation points and trellis edges to find codes with greater free distance. The DC level of each MSM constellation point is computed, then MSM constellation points including the DC distance could be used in the search algorithms. Due to the increased distance between pairs of MSM constellation points, the search algorithms may return codes with larger $d_{\text {free }}$. However, the search with DC distance does not always return better codes due to its sub-optimal nature. In such cases, the codes obtained using the search without DC distance and reported in Table 3.5 are used. Then DC detection is simply used at the receiver without being employed at the construction of trellis codes.

Figures 3.21 and 3.22 plot the performance of best in-band trellis codes with DC detection. The properties of these codes are given in Table 3.6. For comparison the performance of best trellis codes without DC detection presented in Table 3.5 are also shown. An additional average optical power reduction up to $0.50 \mathrm{~dB}$ is achieved with a simultaneous peak power reduction of $0.46 \mathrm{~dB}$ at $\eta=0.75$. However, at some other 


\begin{tabular}{|l|l|l|l|l|l|l|l|l|l|}
\hline & $\eta$ & $W$ & $K$ & $U$ & $M$ & Sub-constellation & $d_{\text {free }}$ & $\rho$ & $\psi$ \\
\hline \multirow{4}{*}{ TC-MSM } & 0.67 & 2 & 2 & 1 & 2 & 5-APSK & 2 & 0.43 & -2.58 \\
\cline { 2 - 10 } With & 0.75 & 3 & 3 & 0 & 4 & 9-APSK & 12 & 0.02 & -1.49 \\
\cline { 2 - 10 } & 1.00 & 3 & 4 & 0 & 4 & 9-APSK & 6.10 & 0.56 & -1.03 \\
\cline { 2 - 10 } & 1.20 & 4 & 6 & 0 & 4 & 9-APSK & 8 & 0.75 & -0.48 \\
\cline { 2 - 10 } & 1.25 & 3 & 5 & 0 & 4 & 9-APSK & 2.59 & 1.74 & 0.11 \\
\cline { 2 - 10 } & 1.40 & 4 & 7 & 1 & 4 & 9-APSK & 1.75 & 2.20 & 0.27 \\
\hline
\end{tabular}

Table 3.6: Properties of TC-MSM codes with DC detection technique(assuming $d_{0}^{2}=$ 1)

frequencies no additional average optical power reduction is achieved. This is because the sub-optimal search algorithm failed to return codes with greater free distance even with DC distance included. Also, the performance of DC detection techniques relies on the variance of DC bias levels for different MSM symbols. If the variance is small, then the distance separation of MSM symbols on the $\phi_{3}(t)$ dimension is small and the additional average optical power reduction is expected to be low.

We also observe in Figure 3.22 that at some bandwidth efficiencies there is an increase in the peak optical power. This is because the codes are picked only according to their average optical power. The additional complexity requirement of DC detection is another matched filter and associated decision devices, which is relatively low. Thus, DC detection technique can be implemented with trellis-coded systems to offer average optical power reduction at small cost.

The performance shown in Figures 3.21 and 3.22 assume an additive white Gaussian noise. However, when the fluorescent light noise introduces a larger noise power at lower frequencies near $\mathrm{DC}$, the performance of $\mathrm{DC}$ detection technique will be degraded. As the power of fluorescent light noise increases, the reduction of both 


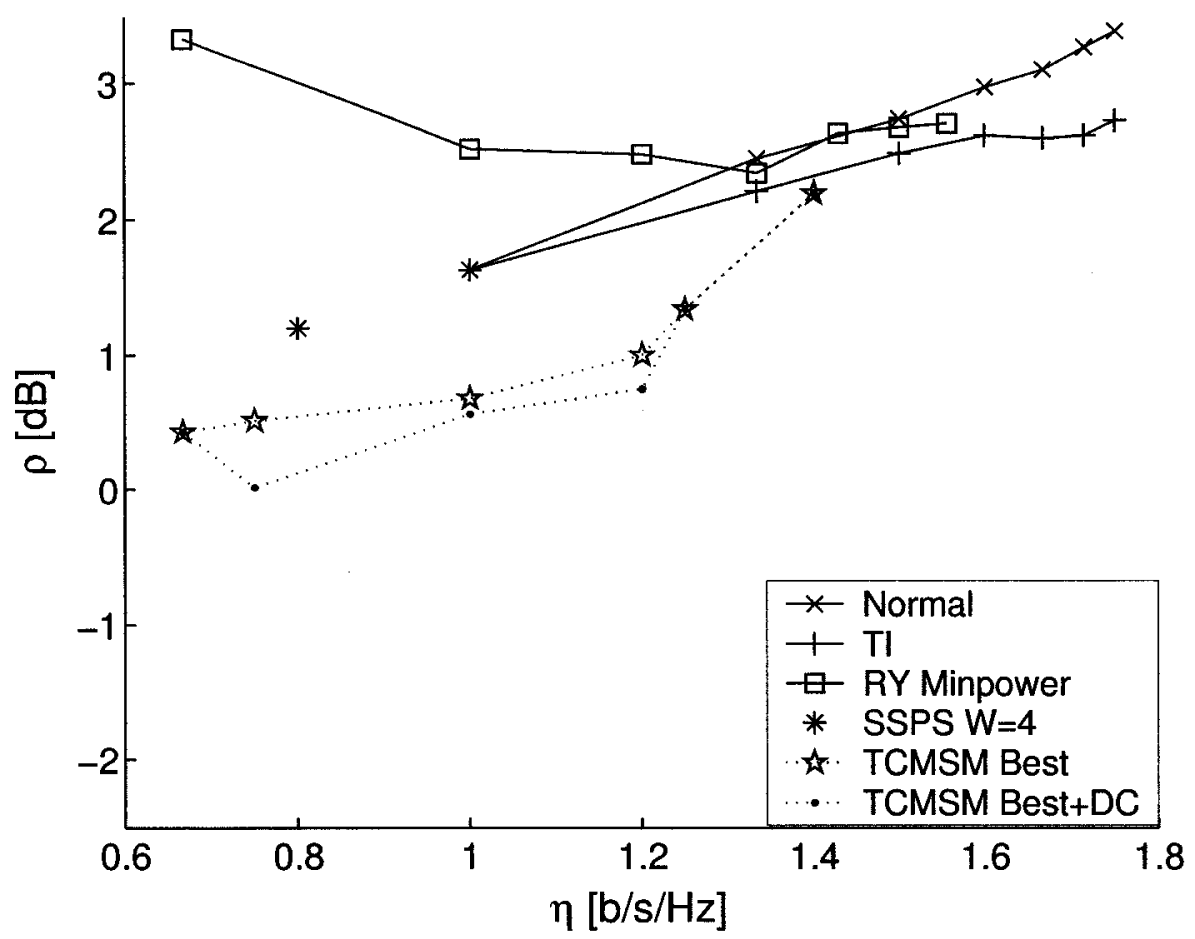

Figure 3.21: Normalized average optical power $(\rho)$ versus bandwidth efficiency $(\eta)$ for in-band trellis coding with DC detection 


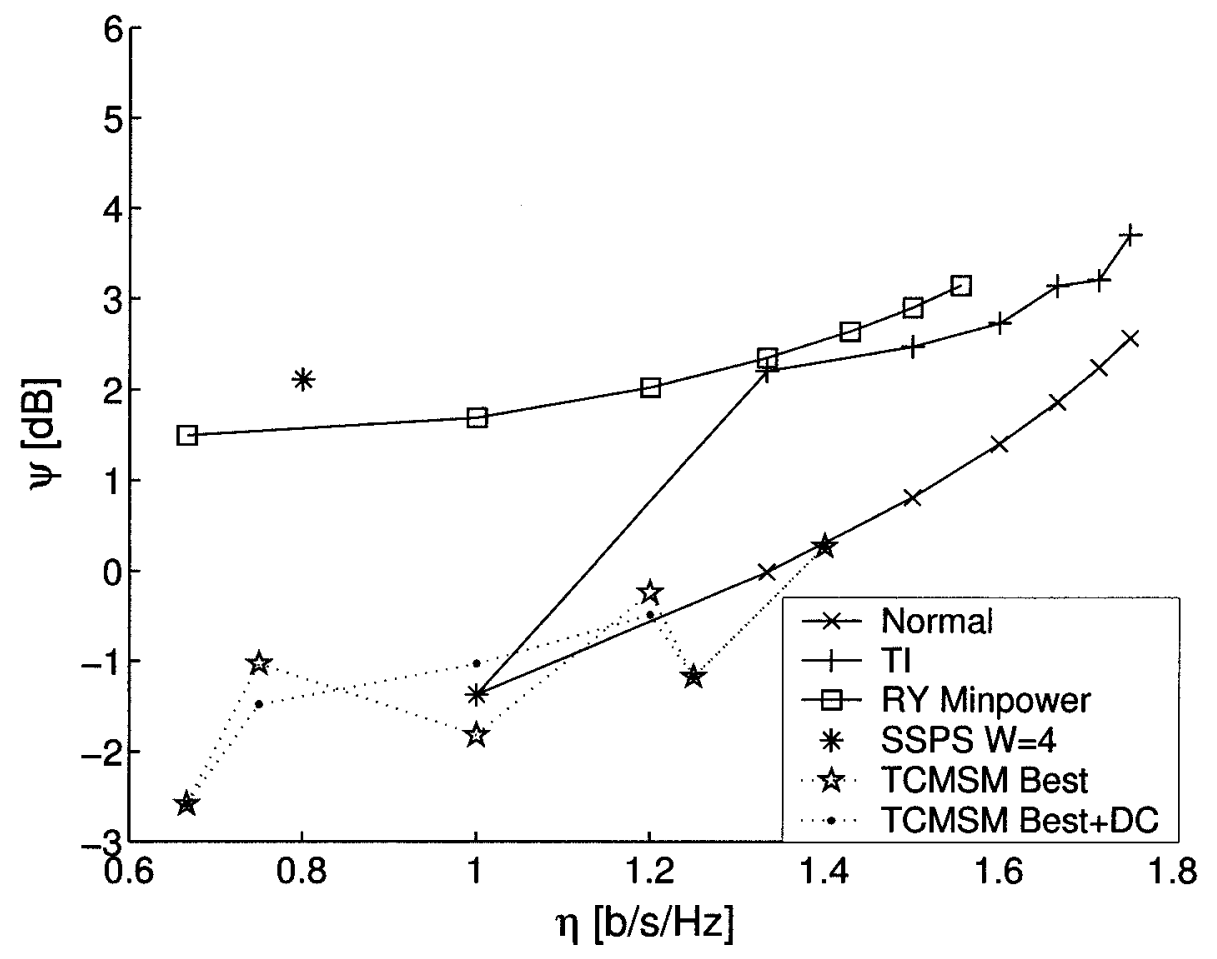

Figure 3.22: Normalized peak optical power $(\psi)$ versus bandwidth efficiency $(\eta)$ for in-band trellis coding with DC detection 
average and peak optical powers obtained from DC detection technique is expected to decrease. In the worst case, the noise at DC bin is dominant and the symbol-bysymbol bias signal cannot be detected. Therefore in the worst case, the performance of MSM wireless optical systems employing DC detection is the same as the systems not employing it. As a result, employing DC detection will not degrade the system performance. Therefore it is desirable to use DC detection technique when the additional complexity at the receiver can be tolerated.

\subsection{Conclusions}

In this section, in-band trellis coding is proposed for MSM wireless optical systems and a reduction in the average optical power as high as $0.95 \mathrm{~dB}$ optical is realized at bandwidth efficiency of 1 . Reduction in the peak optical power up to $0.44 \mathrm{~dB}$ optical is also achieved at selected but not all bandwidth efficiencies. This is because the average optical power is the only criterion in selecting trellis code. In general, the peak optical power of trellis-coded systems can be viewed as not significantly affected comparing to normal MSM systems.

The code design involves constellation expansion, set partitioning and search for a mapping between MSM constellation points and trellis edges. The constellation expansion is done by inserting a zero-amplitude point in QPSK or 8-PSK constellations to allow degrees of freedom in choosing constellation points. Trellis codes are designed over the increased degrees of freedom to realize a reduction in the average optical power. The partitioning of signal set can be done either to the lowest level where only one constellation points exist, or to the level where several constellation points exist within the same partition. Under both partitioning schemes a sub-optimal search procedure is developed to find a good mapping between individual or groups of MSM constellation points and trellis edges to maximize the free Euclidean distance 
of the code. The increased free distance over uncoded systems results in a reduction in the average optical power.

Although in-band trellis coding technique has the advantage of reducing the average optical power while leaving the peak optical power essentially unaffected, additional complexity is required in the form of a trellis coder at the transmitter and a Viterbi decoder at the receiver. As another technique presented in this section, DC detection exploits the signal-space diversity provided by the signal-by-signal bias and achieves up to $0.50 \mathrm{~dB}$ optical reduction in average optical power. Reduction in the peak optical power is also possible at selected bandwidth efficiencies since selection of codes is according to the average optical power only. The performance of DC detection technique depends on the noise power at lower frequencies near DC as well as the variance of DC bias levels of MSM symbols. The additional complexity requirement of this technique is another matched filter and associated decision devices at the receiver, which is low comparing to in-band trellis coding.

In the next chapter, a new approach for adding and exploiting degrees of freedom for average optical power reduction is introduced. The use of out-of-band carriers and its impact on the average and peak optical powers is analyzed using the same metrics. The performance is compared with the techniques presented in this section. Moreover, MSM wireless optical systems combining both out-of-band carriers and inband techniques are presented and are shown to provide the best reduction in average optical power. 


\section{Chapter 4}

\section{Out-of-Band Carrier Design}

In Chapter 3 the MSM constellation is expanded and trellis codes are designed over the increased degrees of freedom to realize a reduction in the average optical power. In this chapter, a different approach for average optical power reduction in MSM wireless optical systems is proposed. The unregulated bandwidth in wireless optical communications offers other degrees of freedom. These degrees of freedom are exploited by adding carriers at out-of-band frequencies and optimizing their amplitudes to reduce the average optical power at the transmitter. These out-of-band carriers are filtered out by the channel, the low-pass filter and digital filter at the receiver. Therefore, they do not affect the bandwidth efficiency of the system.

Since out-of-band carriers are not used for detection, the receiver complexity is not greatly increased. Therefore, this technique shifts the complexity from the receiver to the transmitter, where generation of out-of-band carriers and a lookup table to store the out-of-band carrier amplitudes are required. This is beneficial since wireless optical receivers are typically mobile terminals. Their compact size and limited battery power strictly constrains their complexity and power consumption. In contrast, the transmitter is usually a base station, where additional complexity and power consumption are possible. Note that the out-of-band carrier design technique can not be 
applied in RF systems since their spectrum is strictly limited. The use of out-of-band carriers will cause interference with other ongoing communication schemes.

The problem formulation of average optical power reduction using out-of-band carriers is given in Section 4.1. Two out-of-band carrier amplitude design techniques are introduced and their performance quantified in Section 4.2 and Section 4.3 respectively. The first method optimizes the in-phase and quadrature amplitudes of the outof-band carriers over the set of real numbers using a standard optimization algorithm. Although this technique achieves the best average optical power reduction for adding out-of-band carriers to a particular in-band system, it complicates the transmitter and requires additional memory. The second method optimizes out-of-band carrier amplitudes over a 9-APSK constellation using an exhaustive search. This technique is shown to provide significant average optical power reduction while maintaining a simpler transmitter architecture. In Section 4.4, out-of-band techniques with reduced memory requirement are investigated and shown to trade memory requirements with average optical power reduction capabilities. The DC detection techniques are also applied to out-of-band systems to achieve additional average optical power reduction in Section 4.5 .

\subsection{Problem Formulation}

As discussed earlier in Chapter 1, the infrared region used by wireless optical communications is unregulated worldwide. Moreover, wireless optical emissions are contained in a room, therefore eliminating co-channel interference which is common in RF systems. Therefore using the unregulated bandwidth will not cause interference with other communications schemes. These two features of wireless optical communication make the use of the unregulated bandwidth available to system designers.

However, due to the presence of multipath distortion in diffuse wireless optical 


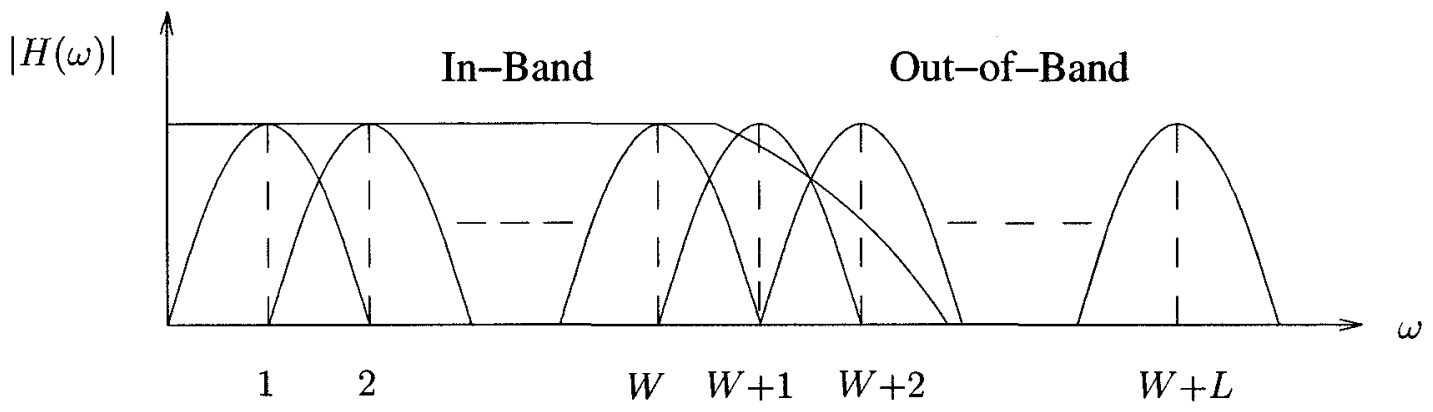

Figure 4.1: Illustration of MSM wireless optical system using $L$ out-of-band carriers systems, the channel at higher frequencies is highly distorted. Traditional approaches neglect this upper bandwidth and send information only at lower frequencies. In our techniques, we also confine data transmission in the lower-frequency in-band spectrum region. However, we use the additional degrees of freedom offered by higher-frequency out-of-band spectrum region to reduce the average optical power requirement. An illustration of this concept is shown in Figure 4.1, where $W$ in-band carriers (carriers 1 to $W$ ) and $L$ out-of-band carriers (carrier $W+1$ to $W+L$ ) are used.

The MSM wireless optical transmitter with $W$ in-band carriers and $L$ out-of-band carriers is shown in Figure 4.2. The conventional MSM wireless optical receiver shown in Figure 2.8 is not greatly changed, although its sampling rate may be increased in an orthogonal frequency-division multiplexing (OFDM) system due to the use of higherfrequency carriers. Following the definitions in (2.21) in Chapter 2, $s(t)$ is given by

$$
s(t)=\sum_{i=1}^{W+L}\left(s_{I i}^{(j)} \phi_{I i}(t-j T)+s_{Q i}^{(j)} \phi_{Q i}(t-j T)\right), t \in[j T,(j+1) T)
$$

As shown in (2.22), a symbol-by-symbol bias is added to $s(t)$ and produces the non-negative electrical signal $x(t)$. Therefore at the $j$ th symbol given a set of inband carrier amplitudes $s_{I i}^{(j)}$ and $s_{Q i}^{(j)}, i \in[1, W]$ as defined in (2.18) and (2.19), our goal is to design higher frequency out-of-band carrier amplitudes $s_{I i}^{(j)}$ and $s_{Q i}^{(j)}$, 


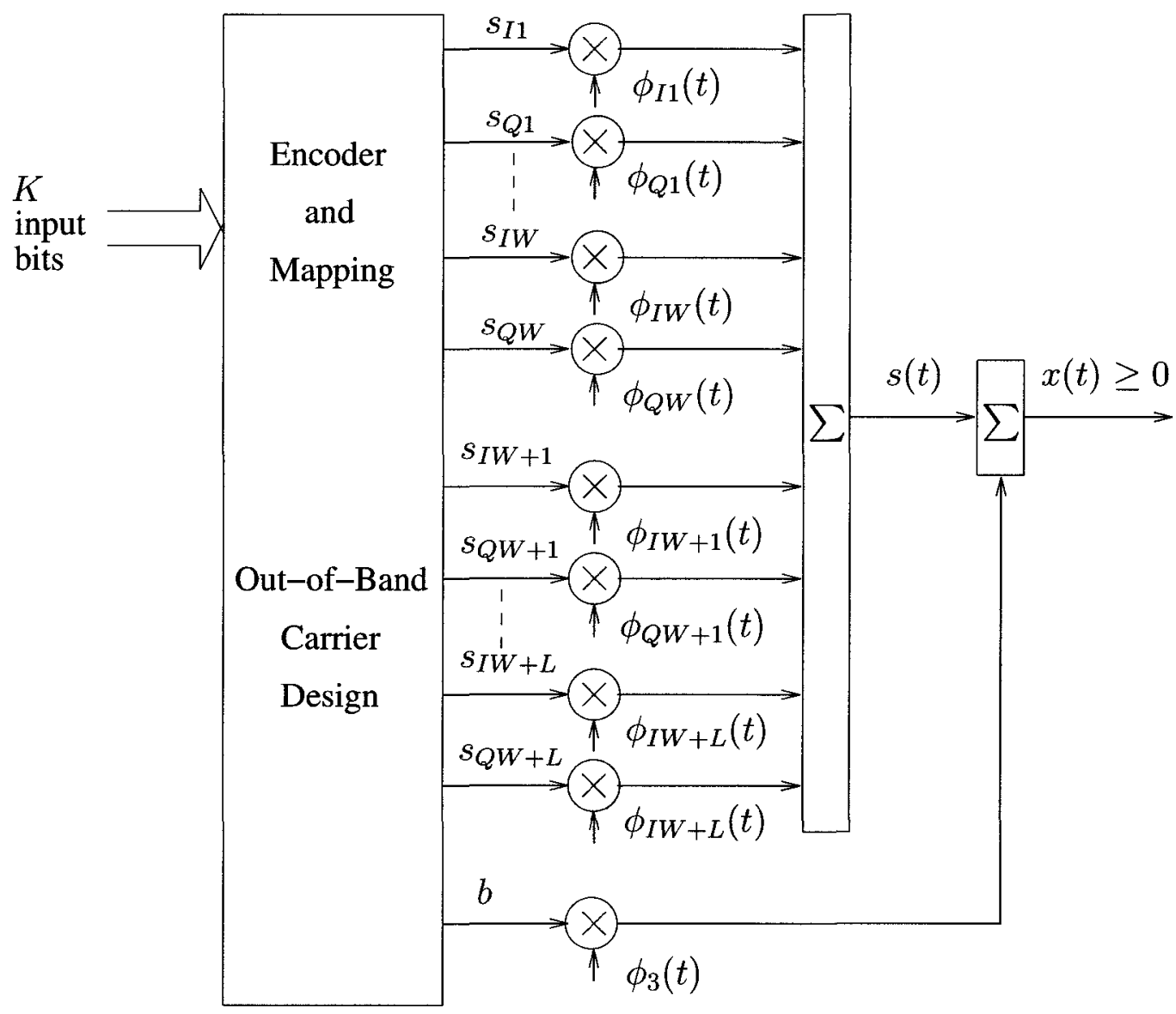

Figure 4.2: The MSM wireless optical transmitter with out-of-band carriers 
$i \in[W+1, W+L]$ such that the bias at that symbol period is minimized. The design is done once for each possible in-band symbol and the results stored in a lookup table at the transmitter. Thus, additional memory is required at the transmitter. In Sections 4.2 and 4.3 , two out-of-band carrier amplitude design techniques are proposed and their performance and complexity quantified.

Another point to notice is that the out-of-band carrier design technique does not change the transmitter architecture of an OFDM system [73], a popular frequencydivision multiplexing (FDM) technique for wireless communications. The block diagram of an OFDM system is shown in Figure 4.3. At the OFDM transmitter the inverse fast Fourier transform (IFFT) is performed on the in-phase and quadrature amplitudes on each frequency carrier to obtain samples of the output waveform. Then a digital-to-analog converter (DAC) coverts the digital samples into a continuous waveform. A guard period, also called cyclic prefix, is added before each symbol to avoid ISI in multipath channels [74]. The received signal first passes a analogto-digital converter (ADC) to produce discrete samples. Then the cyclic prefix is removed and FFT is performed to recover the in-phase and quadrature amplitudes on each carrier. This implementation reduces the circuit complexity as opposed to using multiple frequency up-conversion and down-conversion circuits, as shown in Figure 4.2 and Figure 2.8.

Adding out-of-band carriers in an OFDM transmitter specifies the in-phase and quadrature frequency amplitudes for out-of-band carriers in the frequency-domain data. As a result, the sampling rate at both the transmitter and receiver are increased due to use of higher frequency carriers. The duration of the cyclic prefix is assumed to be small compared to the duration of the data symbol. At the receiver, the cyclic prefix is removed and an FFT is performed on the remaining samples to match filter to the carrier frequencies at each bin in discrete time. Assuming the cyclic prefix is long enough, these bins will remain orthogonal and no inter-channel interference 


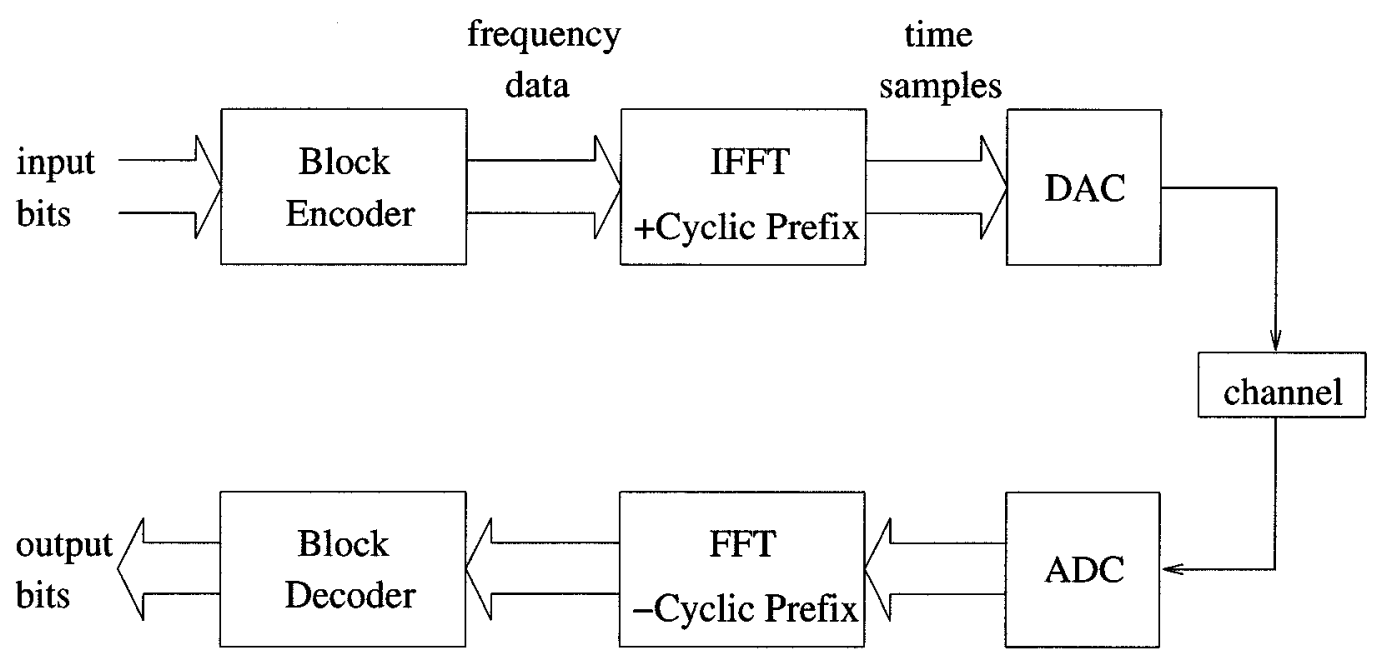

Figure 4.3: Block diagram of an OFDM system

is observed [75]. In this scheme, a full FFT is not required since only the first $(W+1)$ lowpass coefficients are required for detection. In an analog system, a guard frequency band of bandwidth $1 / T \mathrm{~Hz}$ may be inserted between the $W$-th carrier and the $(W+1)$-th carrier. In this way, the separation eases the filtering operation of the $L$ out-of-band carriers and approximately preserves the orthogonality between the carriers.

\subsection{Optimization of Amplitudes Over Real Num- bers}

In this section, we extend the results in [31] and [32] by finding the optimum in-phase and quadrature amplitudes on $L$ out-of-band carriers over the set of real numbers. The formulation of the optimization problem is given in Section 4.2.1 and the performance of this technique is quantified in Section 4.2.2. The minimum amplitude of the continuous waveform is estimated by the minimum amplitude of the discrete samples, therefore estimation error will occur. In Section 4.2 .3 a bound on the estimation error 
is obtained and the error is shown to be negligible. This section concludes in Section 4.2.4. The out-of-band carriers can be applied to any in-band system, and the results obtained in this section represents an upper bound on the achievable average optical power reduction by employing $L$ out-of-band carriers to the chosen in-band system.

\subsubsection{Formulation of the optimization problem}

For each possible selection of data on the $W$ in-band carriers, the problem of finding the $L$ optimum out-of-band carrier amplitudes to reduce the average optical power can be formulated as a convex optimization problem [76]. To estimate the minimum amplitude, at the $j$ th symbol $s^{(j)}(t)$ is discretized into samples $\left\{S_{1}^{(j)}, S_{2}^{(j)}, \ldots S_{F}^{(j)}\right\}$. The choice of $F$ will be discussed in Section 4.2.3. For a given set of in-phase amplitudes $a_{i}^{(j)}$ and quadrature amplitudes $c_{i}^{(j)}$ on $W$ in-band carriers, $i \in[1, W]$, the optimization problem is formulated as follows,

$$
\begin{array}{r}
\text { maximize } S_{\min }^{(j)} \\
\text { subject to } S_{\min }^{(j)} \leq S_{f}^{(j)}, f=1,2 \ldots F \\
s_{I i}^{(j)}=a_{i}^{(j)}, s_{Q i}^{(j)}=c_{i}^{(j)} \quad i \in[1, W] \\
s_{I i}^{(j)} \in \mathbb{R}, s_{Q i}^{(j)} \in \mathbb{R}, \quad i \in[W+1, W+L] \\
S_{f}^{(j)}=\sum_{i=1}^{W+L} s_{I i}^{(j)} \cos \frac{2 \pi i}{T}\left(f \frac{T}{F}\right)+\sum_{i=1}^{W+L} s_{Q i}^{(j)} \sin \frac{2 \pi i}{T}\left(f \frac{T}{F}\right)
\end{array}
$$

This is a linear program and can be solved effectively using standard optimization algorithms. In our simulations the Sedumi 1.05 optimization toolbox [77] was used to solve the linear program for each data symbol. The interior-point method is used and for each data symbol the optimal solution is found within the chosen accuracy, which is typically $10^{-9}$. 
Note the optimization problem must be solved for each data symbol. The results for each data symbol are stored at the transmitter and therefore a lookup table is required to store the real in-phase and quadrature amplitudes on each out-of-band carrier for each data symbol. The lookup table has a size of

$$
n_{\text {input }} \times L
$$

where $n_{\text {input }}$ is the total number of possible input data symbols. Two real numbers are stored at each entry of the table. For a normal MSM system with QPSK modulation employed on each in-band carrier, we have

$$
n_{\text {input }}=4^{W}
$$

Equation 4.4 also holds for a tone injection system. For the trellis-coded systems proposed in Chapter $3, n_{\text {input }}$ is given by (3.10).

The IEEE standard for floating point arithmetic [78] uses 4 bytes for singleprecision floating point numbers, which can represent a smallest number of $2^{-149}$. This precision is more than enough to represent the real amplitudes for the transmitter. Therefore we assume 4 bytes are required for each real amplitude, and 8 bytes are required for each out-of-band carrier since both in-phase and quadrature amplitudes need to be represented. As a result, the memory requirement for either a normal MSM system or a tone injection system with $L$ real-amplitude out-of-band carriers is

$$
4^{W} \times L \times 8 \quad \text { [bytes]. }
$$

The memory requirement for a trellis-coded wireless optical MSM system with $L$ real-amplitude out-of-band carriers is

$$
M \times 2^{K} \times L \times 8 \quad \text { [bytes] }
$$

where $M$ is the number of states for the trellis code and $K$ is the number of input bits sent at each symbol period. The receiver only detects the $W$ in-band data 


\begin{tabular}{|l|l|}
\hline Normal & "Normal" MSM system \\
\hline TI & Tone injection method [66] \\
\hline TCMSM & $\begin{array}{l}\text { Best (minimum average optical power) } \\
\text { in-band trellis codes }\end{array}$ \\
\hline Out-R & $\begin{array}{l}\text { Out-of-Band carriers with real } \\
\text { in-phase and quadrature amplitudes }\end{array}$ \\
\hline Out-9 & $\begin{array}{l}\text { Out-of-Band carriers with 9-APSK } \\
\text { in-phase and quadrature amplitudes }\end{array}$ \\
\hline DC & DC detection technique \\
\hline
\end{tabular}

Table 4.1: Explanation of legends used in performance figures in Chapter 4

subcarriers, therefore applying out-of-band carriers to an in-band system does not change its bandwidth efficiency.

\subsubsection{Performance Analysis}

The real-amplitude out-of-band carrier technique is applied to the normal MSM system, the tone injection system and the best in-band trellis codes presented in Table 3.5. The analytical models discussed in Section 2.6 are used to simulate a working system. As in Section 3.4, all systems are designed to achieve the same bit rate and the same bit error rate of $10^{-6}$. The performance using $L=1$ and $L=4$ out-of-band carriers are shown in Figures 4.4 to 4.7. Normalized average optical power $\rho$ defined in (2.37) and normalized peak optical power $\psi$ defined in (2.38) are plotted versus bandwidth efficiency $\eta$, defined in (2.30). The performance using $L=2$ and $L=3$ real-amplitude out-of-band carriers are presented in the Appendix. An explanation of the legends used in figures within this chapter is given in Table 4.1.

It is observed that by employing out-of-band carriers to in-band systems, the 


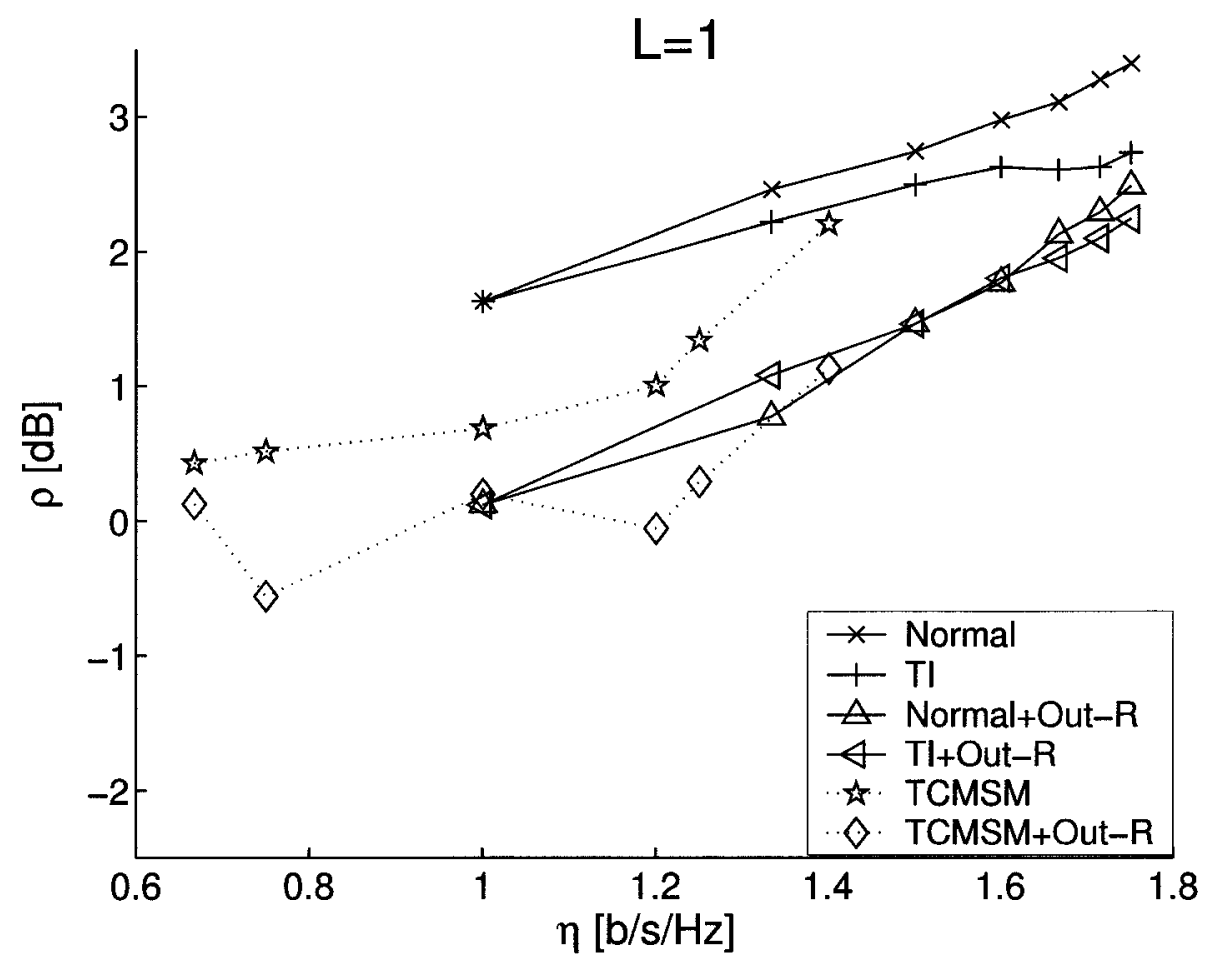

Figure 4.4: Normalized average optical power $(\rho)$ versus bandwidth efficiency $(\eta)$ for real-amplitude $L=1$ out-of-band techniques 


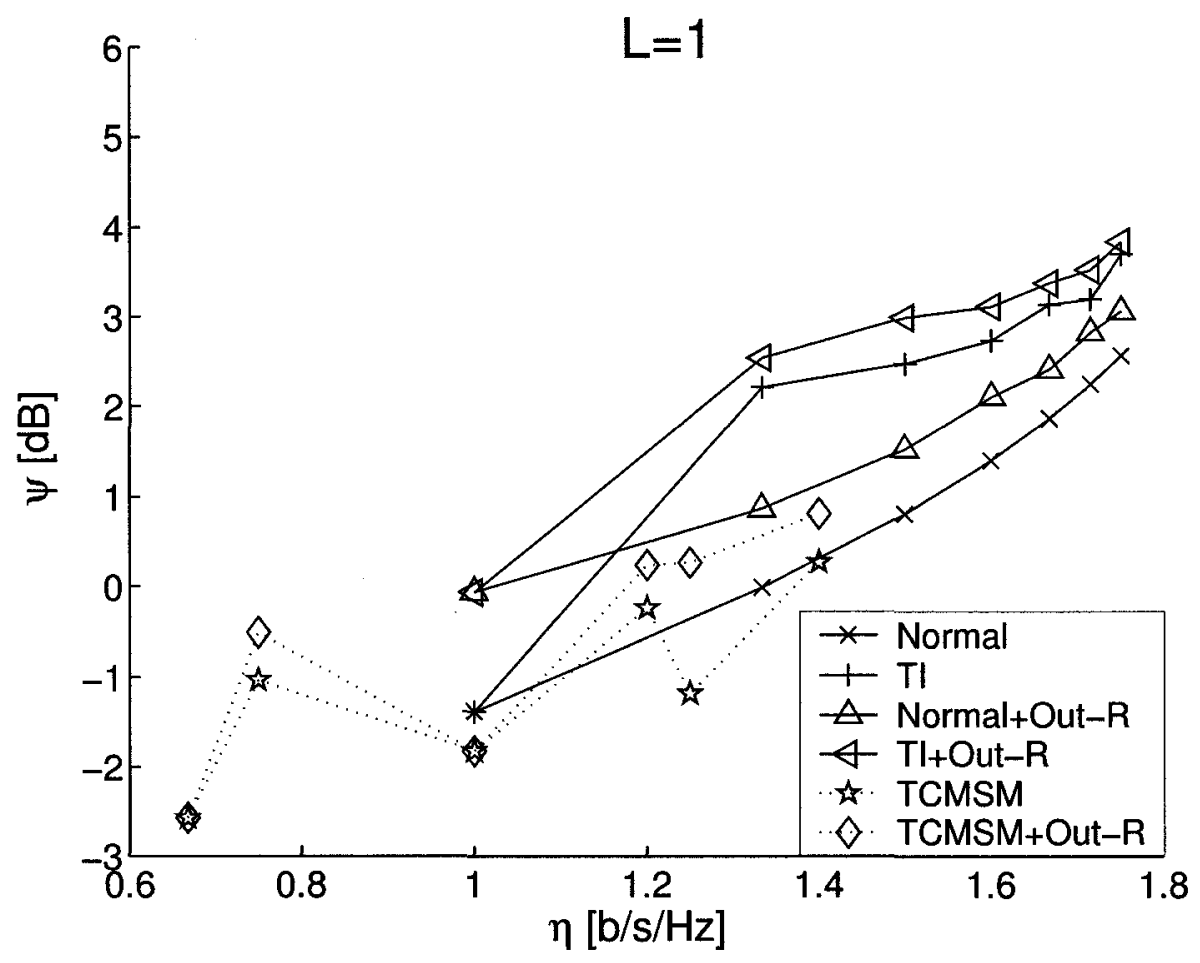

Figure 4.5: Normalized peak optical power $(\psi)$ versus bandwidth efficiency $(\eta)$ for real-amplitude $L=1$ out-of-band techniques 


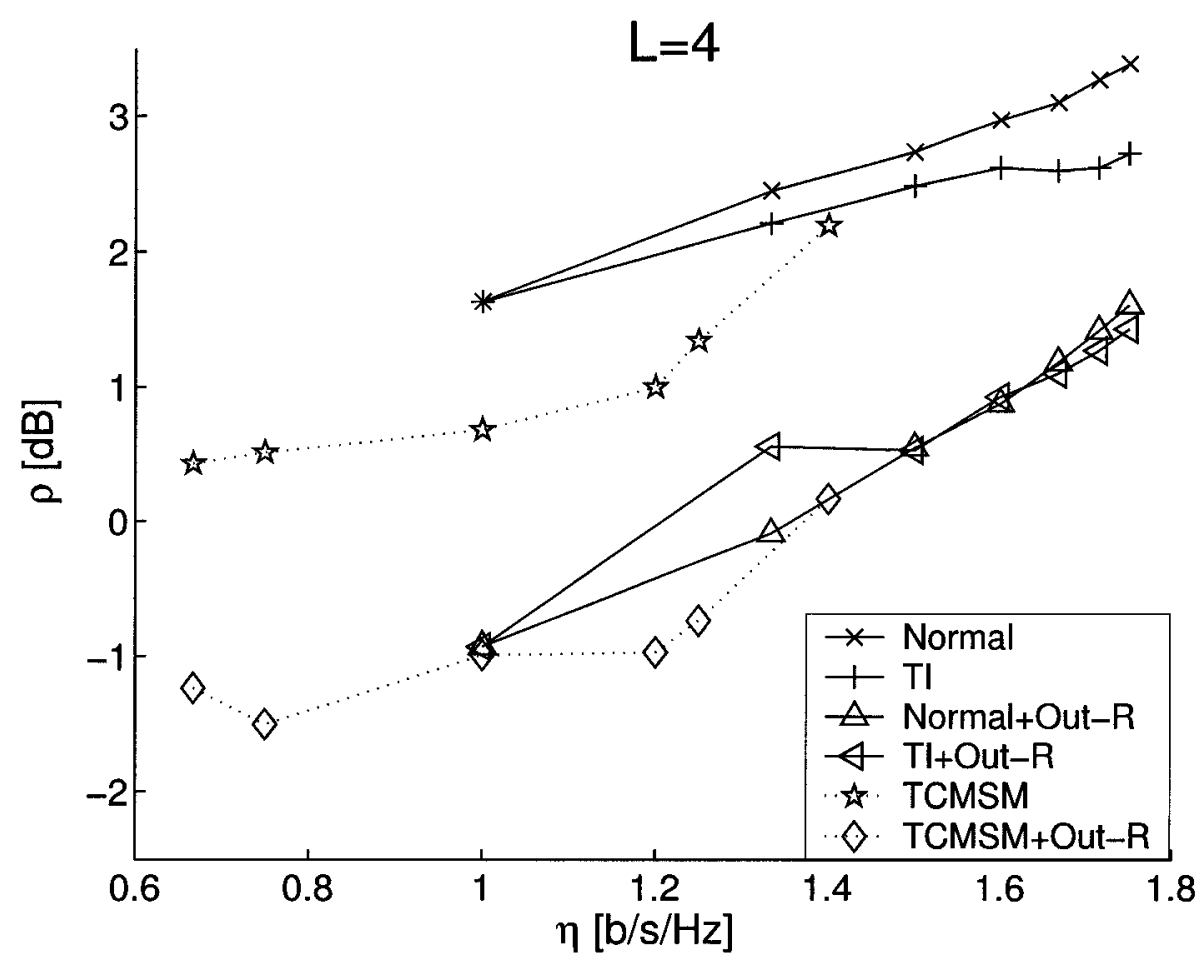

Figure 4.6: Normalized average optical power $(\rho)$ versus bandwidth efficiency $(\eta)$ for real-amplitude $L=4$ out-of-band techniques 


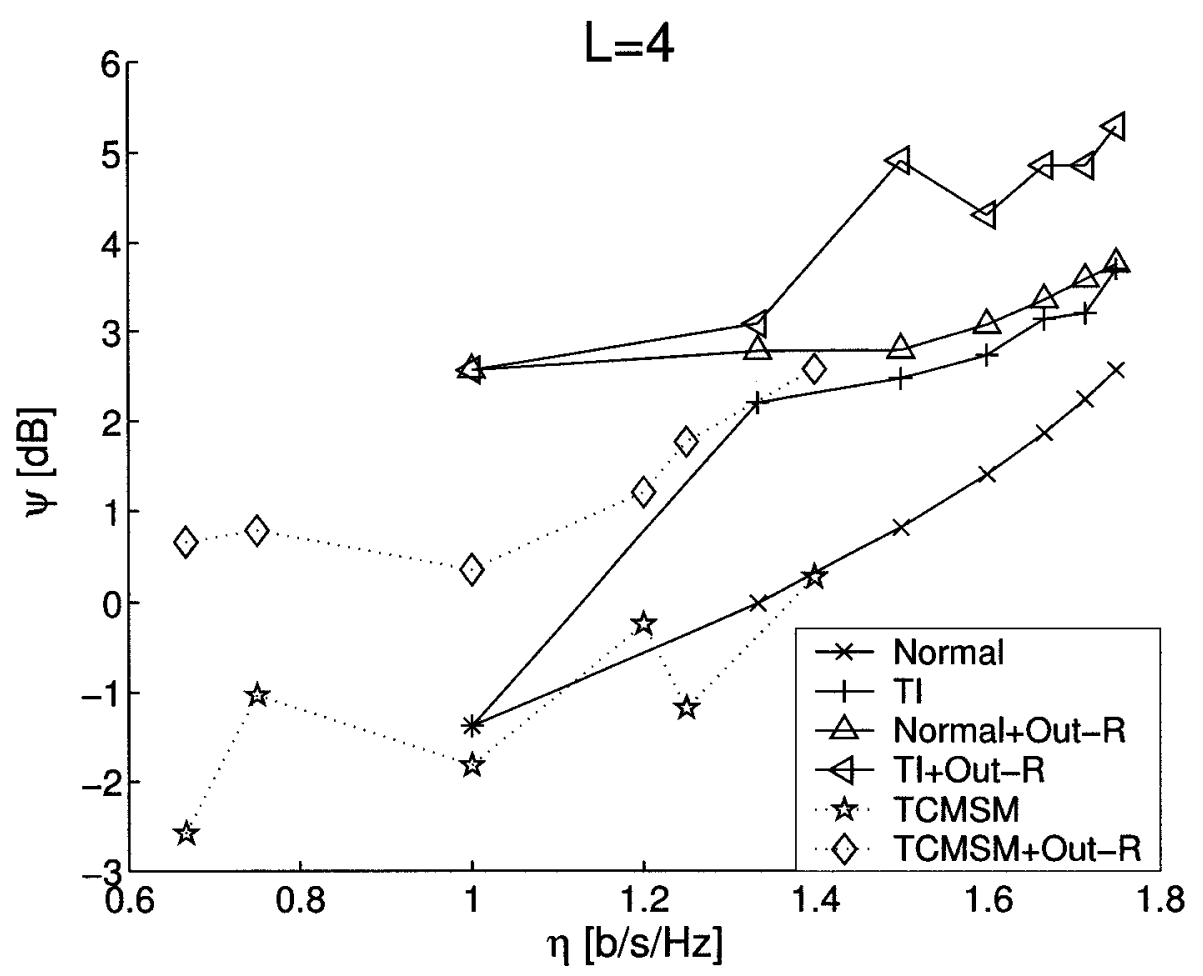

Figure 4.7: Normalized peak optical power $(\psi)$ versus bandwidth efficiency $(\eta)$ for real-amplitude $L=4$ out-of-band techniques 
average optical power is reduced while the peak optical power is increased over all bandwidth efficiencies. This is because the out-of-band carriers are designed to reduce the average optical power only, and the peak optical power is not included in the design objective. To characterize the average optical power reduction, the minimum reduction and maximum reduction, as well as the average reduction over all bandwidth efficiencies are measured. Notice the reductions measured are achieved by employing out-of-band carriers to the in-band systems. That is, we compare the average optical power of the in-band system applied with out-of-band carriers to that of the in-band system alone. Similarly, the minimum, maximum and average increase in the peak optical power caused by out-of-band carriers are measured.

By applying $L=1$ real-amplitude out-of-band carrier to a normal MSM system, the average optical reduction ranges from $0.91 \mathrm{~dB}$ to $1.51 \mathrm{~dB}$ optical, while the peak optical increase ranges from $0.50 \mathrm{~dB}$ to $1.32 \mathrm{~dB}$ optical for different bandwidth efficiencies. On average, $1.22 \mathrm{~dB}$ of average optical power reduction is realized with a peak optical power increase of $0.75 \mathrm{~dB}$. In contrast, by applying $L=1$ real-amplitude out-of-band carrier to the tone injection system and the best in-band trellis codes in Section 3.5, the average optical power reductions are $0.88 \mathrm{~dB}$ optical and $0.84 \mathrm{~dB}$ optical on average respectively, and the increases in peak optical power are $0.47 \mathrm{~dB}$ optical and $0.50 \mathrm{~dB}$ optical on average.

Increasing the number of real-amplitude out-of-band carriers will further reduce the average optical power at the cost of increased peak optical power and increased memory requirement. On average, $L=4$ real-amplitude out-of-band carriers achieve an optical power reduction of $2.14 \mathrm{~dB}$ at the cost of increased peak optical power of $2.06 \mathrm{~dB}$ for normal MSM systems. When applying to tone injection system and inband trellis codes, $1.72 \mathrm{~dB}$ and $1.91 \mathrm{~dB}$ of average optical power reduction is realized on average respectively, with peak optical power increases of $1.97 \mathrm{~dB}$ and $2.32 \mathrm{~dB}$ on average respectively. The average optical power reduction, peak optical power 
increase as well as the additional memory requirement for applying real-amplitude out-of-band carriers to in-band systems are detailed in Tables 4.2 and 4.3 for $L=1$ and $L=4$ respectively. The details for using $L=2$ and $L=3$ real-amplitude outof-band carriers are included in the Appendix and the performance are found to be between those of $L=1$ and $L=4$.

It is observed that at lower bandwidth efficiencies, in-band trellis coding with realamplitude out-of-band carriers has lower average optical power requirement than normal MSM system with real-amplitude out-of-band carriers. However, normal MSM systems with out-of-band carriers are capable of operating at higher bandwidth efficiencies. Moreover, such systems employ symbol-by-symbol maximum likelihood detection and therefore have low receiver complexity. In contrast, trellis-coded systems require the use of a Viterbi decoder at the receiver and has higher receiver complexity.

\subsubsection{Bounding the estimation error}

One issue of the real-amplitude out-of-band carrier technique is that the estimation of minimum amplitude of $s(t)$ by sampling must be close to the true minimum. To guarantee that, the sampling rate must be high enough. In other words, the value of $F$ in (4.2) should be set high enough to ensure that the error in estimating the amplitudes is tolerable. To bound the estimation error, we first derive an upper bound on the absolute value of the slope between any two samples of a bandwidth-limited continuous waveform. Then, we get an upper bound as well as a lower bound on the amplitude of the continuous waveform at time periods between any two samples. Comparing the bounds on the amplitudes among all pairs of adjacent samples, we get the upper bound and lower bound for the continuous waveform during the time period when the samples are taken. 


\begin{tabular}{|c|c|c|c|c|}
\hline In-band & $\eta$ & Reduction of $\rho[\mathrm{dB}]$ & Increase in $\psi[\mathrm{dB}]$ & Memory [bytes] \\
\hline \multirow[t]{7}{*}{ Normal } & 1.00 & 1.51 & 1.32 & 32 \\
\hline & 1.33 & 1.68 & 0.88 & 128 \\
\hline & 1.50 & 1.27 & 0.72 & 512 \\
\hline & 1.60 & 1.21 & 0.70 & 2048 \\
\hline & 1.67 & 0.98 & 0.55 & 8192 \\
\hline & 1.71 & 0.99 & 0.58 & 32768 \\
\hline & 1.75 & 0.91 & 0.50 & 131072 \\
\hline \multirow[t]{7}{*}{$\mathrm{TI}$} & 1.00 & 1.51 & 1.32 & 32 \\
\hline & 1.33 & 1.13 & 0.33 & 128 \\
\hline & 1.50 & 1.03 & 0.52 & 512 \\
\hline & 1.60 & 0.82 & 0.38 & 2048 \\
\hline & 1.67 & 0.65 & 0.25 & 8192 \\
\hline & 1.71 & 0.53 & 0.33 & 32768 \\
\hline & 1.75 & 0.49 & 0.14 & 131072 \\
\hline \multirow[t]{6}{*}{ TCMSM } & 0.67 & 0.30 & 0.00 & 64 \\
\hline & 0.75 & 1.07 & 0.52 & 256 \\
\hline & 1.00 & 0.48 & 0.00 & 1024 \\
\hline & 1.20 & 1.05 & 0.48 & 2048 \\
\hline & 1.25 & 1.05 & 1.44 & 1024 \\
\hline & 1.40 & 1.07 & 0.54 & 4096 \\
\hline
\end{tabular}

Table 4.2: Performance of $L=1$ real-amplitude out-of-band carriers applied to normal MSM systems, tone injection systems and the best in-band trellis codes 


\begin{tabular}{|c|c|c|c|c|}
\hline In-band & $\eta$ & Reduction of $\rho[\mathrm{dB}]$ & Increase in $\psi[\mathrm{dB}]$ & Memory [bytes] \\
\hline \multirow[t]{7}{*}{ Normal } & 1.00 & 2.56 & 3.96 & 128 \\
\hline & 1.33 & 2.54 & 2.80 & 512 \\
\hline & 1.50 & 2.19 & 1.99 & 2048 \\
\hline & 1.60 & 2.09 & 1.67 & 8192 \\
\hline & 1.67 & 1.92 & 1.49 & 32768 \\
\hline & 1.71 & 1.86 & 1.34 & 131072 \\
\hline & 1.75 & 1.79 & 1.19 & 524288 \\
\hline \multirow[t]{7}{*}{ TI } & 1.00 & 2.56 & 3.96 & 128 \\
\hline & 1.33 & 1.65 & 0.88 & 512 \\
\hline & 1.50 & 1.96 & 2.44 & 2048 \\
\hline & 1.60 & 1.69 & 1.57 & 8192 \\
\hline & 1.67 & 1.50 & 1.72 & 32768 \\
\hline & 1.71 & 1.36 & 1.66 & 131072 \\
\hline & 1.75 & 1.30 & 1.57 & 524288 \\
\hline \multirow[t]{6}{*}{ TCMSM } & 0.67 & 1.66 & 3.23 & 256 \\
\hline & 0.75 & 2.02 & 1.81 & 1024 \\
\hline & 1.00 & 1.67 & 2.18 & 4096 \\
\hline & 1.20 & 1.97 & 1.45 & 8192 \\
\hline & 1.25 & 2.08 & 2.95 & 4096 \\
\hline & 1.40 & 2.03 & 2.31 & 16384 \\
\hline
\end{tabular}

Table 4.3: Performance of $L=4$ real-amplitude out-of-band carriers applied to normal MSM systems, tone injection systems and the best in-band trellis codes 
The Fourier transform is given by

$$
\begin{aligned}
S(\omega) & =\int_{-\infty}^{+\infty} s(t) e^{-j \omega t} d t \\
s(t) & =\frac{1}{2 \pi} \int_{-\infty}^{+\infty} S(\omega) e^{j \omega t} d \omega
\end{aligned}
$$

According to Triangle Inequality for Integration, we have

$$
|s(t)| \leq \frac{1}{2 \pi} \int_{-\infty}^{+\infty}\left|S(\omega) e^{j \omega t}\right| d \omega \leq \frac{1}{2 \pi} \int_{-\infty}^{+\infty}|S(\omega)| d \omega
$$

since $\left|e^{j \omega t}\right|=1$. The above inequality holds when the integral exists, which is true in the cases considered in this section.

According to the the differentiation property of Fourier transform,

$$
s^{\prime}(t) \leftrightarrow j \omega S(\omega)
$$

where $s^{\prime}(t)$ is the derivative of $s(t)$. Therefore,

$$
\left|s^{\prime}(t)\right| \leq \frac{1}{2 \pi} \int_{-\infty}^{+\infty}|\omega S(\omega)| d \omega .
$$

At the $k$ th symbol, the time signal is

$$
s^{(k)}(t)=\sum_{i=1}^{W+L}\left(s_{I i}^{(k)} \cos \omega_{i} t+s_{Q i}^{(k)} \sin \omega_{i} t\right), \quad t \in[k T,(k+1) T)
$$

where $T$ is the symbol period. The corresponding frequency domain signal in that period is

$$
S^{(k)}(\omega)=\sum_{i=1}^{W+L} \pi\left(\left(s_{I i}^{(k)}-j s_{Q i}^{(k)}\right) \delta\left(\omega-\omega_{i}\right)+\left(s_{I i}^{(k)}+j s_{Q i}^{(k)}\right) \delta\left(\omega+\omega_{i}\right)\right)
$$

Therefore,

$$
\begin{aligned}
\frac{1}{2 \pi} \int_{-\infty}^{+\infty}\left|\omega S^{(k)}(\omega)\right| d \omega & =\frac{1}{2} \sum_{i=1}^{W+L}\left|\left(s_{I i}^{(k)}-j s_{Q i}^{(k)}\right) \omega_{i}\right|+\frac{1}{2} \sum_{i=1}^{W+L}\left|\left(s_{I i}^{(k)}+j s_{Q i}^{(k)}\right) \omega_{i}\right| \\
& =\sum_{i=1}^{W+L}\left|\left(s_{I i}^{(k)}+j s_{Q i}^{(k)}\right) \omega_{i}\right|
\end{aligned}
$$


Using Equations (4.9) and (4.12), for the $k$ th symbol we have

$$
\left|s^{\prime(k)}(t)\right| \leq \sum_{i=1}^{W+L}\left|\left(s_{I i}^{(k)}+j s_{Q i}^{(k)}\right) \omega_{i}\right| \quad t \in[k T,(k+1) T)
$$

Equation (4.13) is an upper bound on the absolute value of the slope for the continuous waveform. Therefore at the $k$ th symbol, let $r$ denote the upper bound on the absolute value of the slope, i.e.,

$$
r=\sum_{i=1}^{W+L}\left|\left(s_{I i}^{(k)}+j s_{Q i}^{(k)}\right) \omega_{i}\right| \quad t \in[k T,(k+1) T) .
$$

As shown in Figure 4.8, the $k$ th symbol starts at time $t_{0}$ and ends at $t_{0}+T$. Let $t_{1}$ and $t_{2}$ denote two adjacent sample points within the $k$ th symbol. Then as shown in Figure 4.8, the function $s^{(k)}(t)$ must lie above the line segment $l_{1}$ with slope $-r$ for $t_{1} \leq t \leq t_{0}+T$, and above line segment $l_{2}$ with slope $r$ for $t_{0} \leq t \leq t_{2} . t_{0}$ To prove this, notice that

$$
s^{(k)}(t)-s^{(k)}\left(t_{1}\right)=\int_{t_{1}}^{t} s^{\prime(k)}(\hat{t}) d \hat{t} \geq \int_{t_{1}}^{t}-r d \hat{t}=-r\left(t-t_{1}\right) \quad t_{1} \leq t \leq t_{0}+T
$$

Re-arranging (4.15) gives

$$
s^{(k)}(t) \geq s^{(k)}\left(t_{1}\right)-r\left(t-t_{1}\right), \quad t_{1} \leq t \leq t_{0}+T
$$

Equation (4.16) proves that $s^{(k)}(t)$ must lie above the line segment $l_{1}$ as illustrated in Figure 4.8. Similarly, we have

$$
s^{(k)}\left(t_{2}\right)-s^{(k)}(t)=\int_{t}^{t_{2}} s^{\prime(k)}(\hat{t}) d \hat{t} \leq \int_{t}^{t_{2}} r d \hat{t}=r\left(t_{2}-t\right) \quad t_{0} \leq t \leq t_{2}
$$

Re-arranging (4.17) gives

$$
s^{(k)}(t) \geq s^{(k)}\left(t_{2}\right)+r\left(t-t_{2}\right), \quad t_{0} \leq t \leq t_{2}
$$

which proves that $s^{(k)}(t)$ must lie above the line segment $l_{2}$ as illustrated in Figure 4.8. 


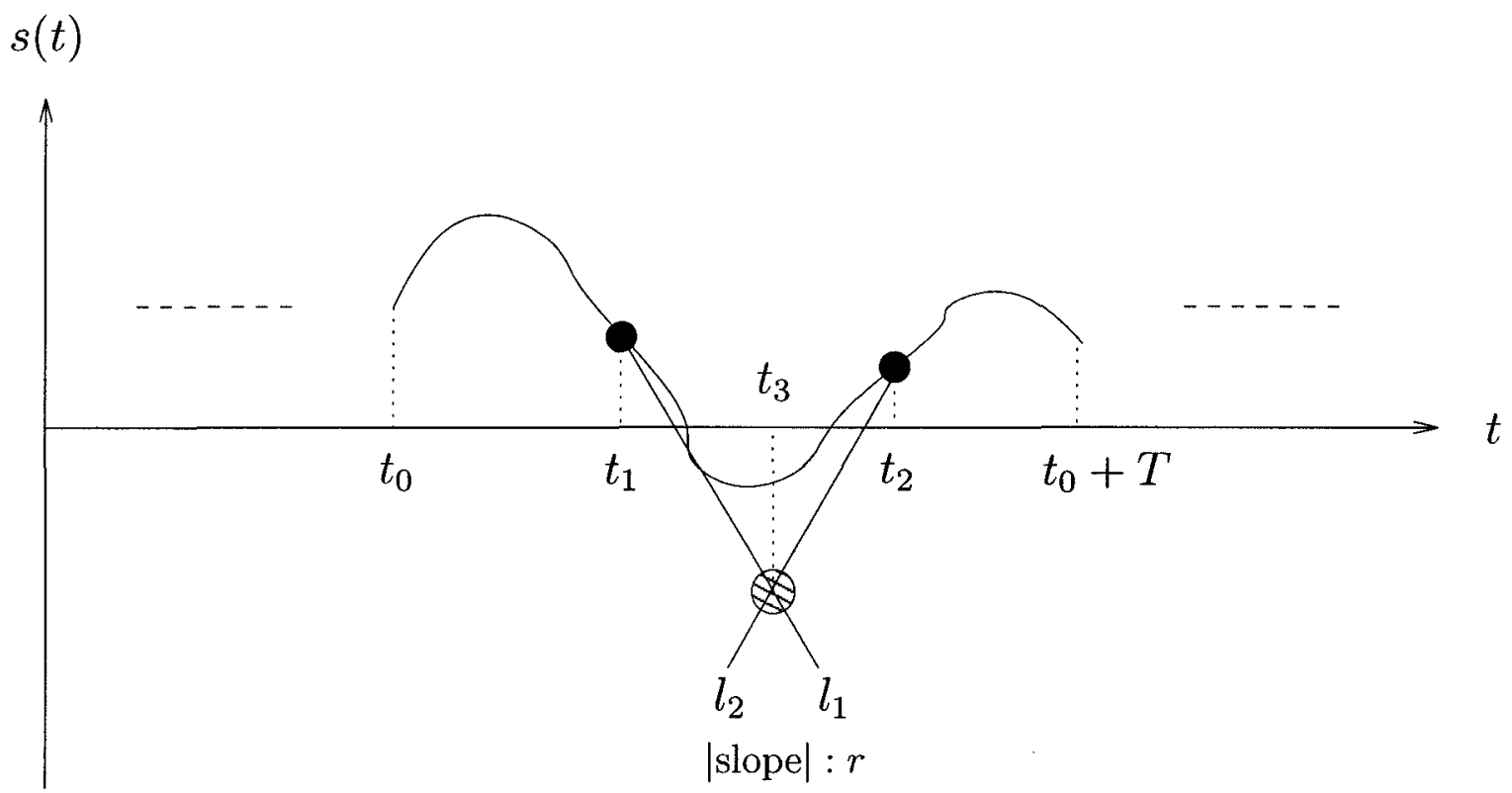

Figure 4.8: Illustration of the minimum possible amplitude between two sample points

Using both (4.16) and (4.18), we can find the minimum possible amplitude point $\left(t_{3}, s^{(k)}\left(t_{3}\right)\right)$ located within time period $t_{1} \leq t \leq t_{2}$. As shown in Figure 4.8, the following relationships must be satisfied

$$
\begin{aligned}
& \frac{s^{(k)}\left(t_{3}\right)-s^{(k)}\left(t_{1}\right)}{t_{3}-t_{1}}=-r \\
& \frac{s^{(k)}\left(t_{2}\right)-s^{(k)}\left(t_{3}\right)}{t_{2}-t_{3}}=r
\end{aligned}
$$

Solving the equations, we get

$$
s^{(k)}\left(t_{3}\right)=\frac{s^{(k)}\left(t_{1}\right)+s^{(k)}\left(t_{2}\right)-r\left(t_{2}-t_{1}\right)}{2}=\frac{s^{(k)}\left(t_{1}\right)+s^{(k)}\left(t_{2}\right)-r T / F}{2}
$$

where $T / F$ is the sample period. Therefore calculating equation (4.21) among all pairs of adjacent samples within this symbol period and picking the minimum $s^{(k)}\left(t_{3}\right)$ value gives a lower bound on the minimum amplitude of the during this symbol. Comparing the lower bounds among all symbols gives a lower bound on the minimum amplitude of the transmitted MSM waveform. 


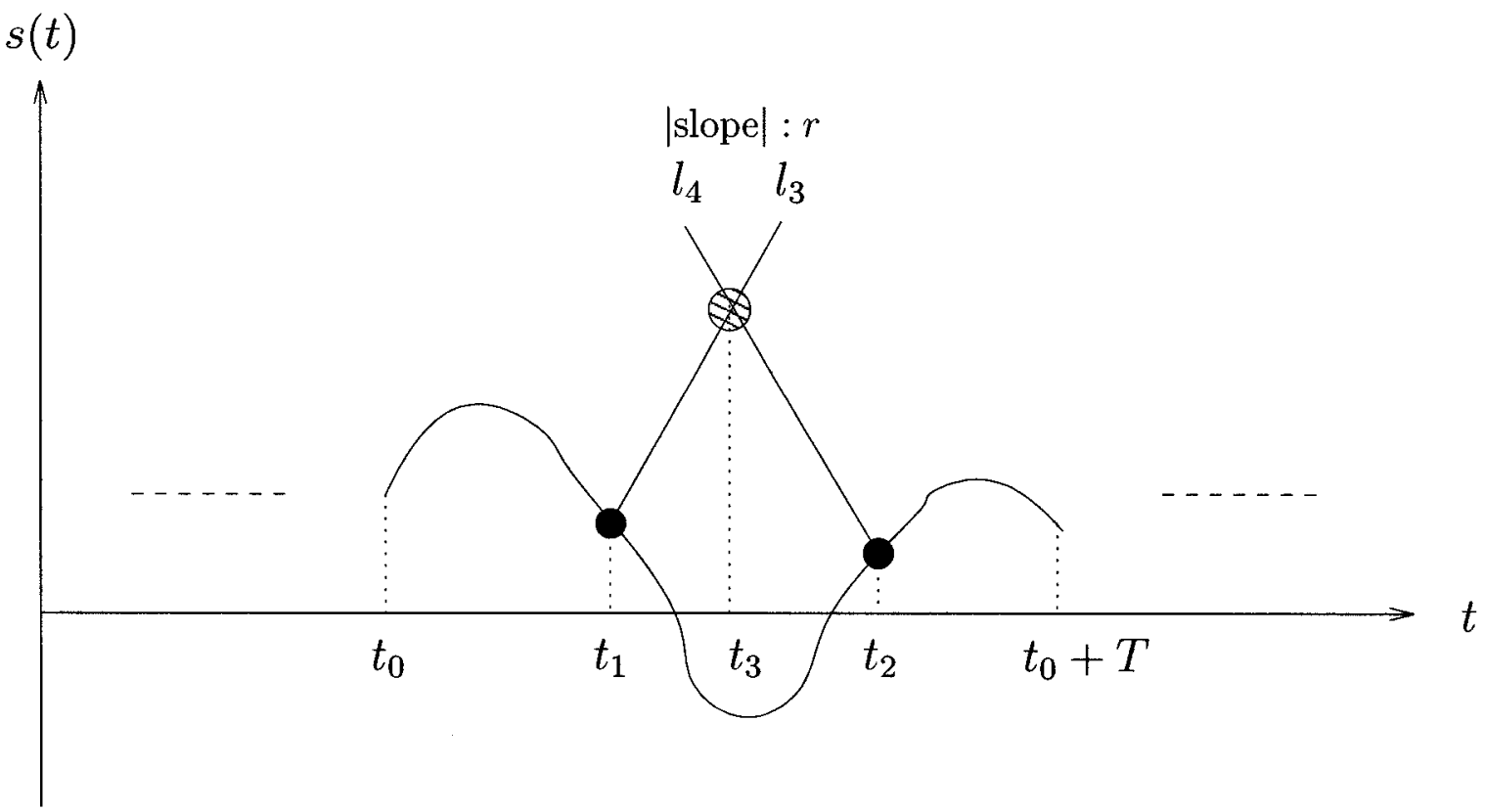

Figure 4.9: Illustration of the maximum possible amplitude between two sample points

Following the same argument, one can prove that the function $s^{(k)}(t)$ must lie below the line segment $l_{3}$ with slope $r$ for $t_{1} \leq t \leq t_{0}+T$, and below line segment $l_{4}$ with slope $r$ for $t_{0} \leq t \leq t_{2}$, as illustrated in Figure 4.9. The points must satisfy

$$
\begin{aligned}
& \frac{s^{(k)}\left(t_{3}\right)-s^{(k)}\left(t_{1}\right)}{t_{3}-t_{1}}=r \\
& \frac{s^{(k)}\left(t_{2}\right)-s^{(k)}\left(t_{3}\right)}{t_{2}-t_{3}}=-r
\end{aligned}
$$

Solving the equations, we get

$$
s^{(k)}\left(t_{3}\right)=\frac{s^{(k)}\left(t_{1}\right)+s^{(k)}\left(t_{2}\right)+r T / F}{2}
$$

Calculating equation (4.24) among all pairs of adjacent samples within all symbol periods and picking the maximum value gives an upper bound on the maximum amplitude of the transmitted MSM waveform. 
These upper bounds and lower bounds on the amplitude are compared to the estimated minimum and maximum amplitudes presented in Figures 4.4 to 4.7, where $F$ is set to 1000. Although the bounds derived in this section may be loose, the difference between the estimated $\rho, \psi$ and the bounds on them is at most $0.01 \mathrm{~dB}$. Thus the estimation error occurred by using samples of the continuous waveform can be neglected.

\subsubsection{Conclusions}

In this section, the in-phase and quadrature amplitudes of the out-of-band carriers are optimized over the set of real numbers using a standard optimization algorithm. The real-amplitude out-of-carriers can be applied to any in-band system and up to $2.56 \mathrm{~dB}$ of average optical power reduction is achieved by using $L=4$ out-of-band carriers. However, the cost of this technique is an increase in the peak optical power as high as $3.96 \mathrm{~dB}$ and additional memory requirement ranging from 32 bytes to 524288 bytes, depending on the in-band system and $L$. Although the transmitter complex is increased, this technique demonstrates an upper bound on the achievable average optical power reduction by employing $L$ out-of-band carriers to a chosen in-band system.

\subsection{Optimization of Amplitudes Over a Discrete Constellation}

To reduce the transmitter complexity and the additional memory requirements, in Section 4.3.1 out-of-band carrier amplitudes are designed over a discrete constellation. It is shown in Section 4.3.2 that although discrete-constellation out-of-band technique achieve a smaller average optical power reduction comparing to real-amplitude 
out-of-band carriers, both the peak optical power increase and additional memory requirement are reduced.

\subsubsection{Optimization by an Exhaustive Search}

The 9-APSK constellation presented in Figure 3.8 is employed by each of the $L$ out-ofband carriers. Amplitudes are chosen from this discrete set rather than real numbers. To choose the best amplitudes jointly over $L$ out-of-band carriers to minimize the average optical power, an exhaustive search over $9^{L}$ possible amplitudes is performed once for each data symbol and the results stored in a lookup table at the transmitter. The exhaustive search is implemented over a total number of

$$
n_{\text {input }} \times 9^{L}
$$

where $n_{\text {input }}$ is given in (4.4) for normal MSM system and tone injection system, and given in (3.10) for trellis-coded systems proposed in Chapter 3.

Note that each 9-APSK amplitude requires half a byte to represent, and each outof-band carrier requires 1 byte to store both in-phase and quadrature amplitudes. Therefore, the memory requirement for either a normal MSM system or a tone injection system with $L$ discrete-constellation out-of-band carriers is

$$
4^{W} \times L \quad \text { [bytes] }
$$

The memory requirement for a trellis-coded wireless optical MSM system with $L$ discrete-constellation out-of-band carriers is

$$
\left.M \times 2^{K} \times L \quad \text { [bytes }\right]
$$

Comparing (4.26) and (4.27) with (4.5) and (4.6) in Section 4.2.1, we notice that the memory required by discrete-constellation out-of-band carriers is only $1 / 8$ of the memory required by real-amplitude out-of-band carriers. Therefore the additional 
complexity of the transmitter for discrete-constellation out-of-band carriers is much less.

The above discussed method is simply a special case of You's reserved subcarrier block coding [31] by using 9-APSK subconstellation and making all reserved subcarriers out-of-band, which has the benefit of leaving the bandwidth efficiency of the system unaffected. However, to further improve the average optical power reduction capability, we introduce a scaling factor $\alpha$ in the range of $(0,1]$ to scale the amplitude transmitted on all out-of-band carriers with respect to the amplitudes of the in-band carriers. Unlike the most general case of individual real-numbered amplitudes on each out-of-band carrier considered in Section 4.2, $\alpha$ is fixed for all symbols.

To find the best value of $\alpha$, the range of $(0,1]$ is discretized into 20 points. For each $\alpha$ the exhaustive search in (4.25) is implemented to minimize the average optical power. Therefore the total number of searches is now

$$
20 \times n_{\text {input }} \times 9^{L}
$$

By introducing $\alpha$, the size of the lookup table is increased by 4 bytes. Moreover, the search time is increased. The gain achieved by changing $\alpha$ for out-of-band carriers is quantified in Figure 4.10 for one of the best in-band trellis codes operating at $\eta=1$ in Figure 4.6. The normalized average optical power requirement to achieve a BER of $10^{-6}$ is plotted versus $\alpha$, which is discretized into 100 points in this figure for illustration purpose. The performance realized when optimizing out-of-band carrier amplitudes over real number as in Section 4.2 is also presented. An additional $0.57 \mathrm{~dB}$ reduction of $\rho$ is realized from setting $\alpha=1$ in the design of 9-APSK out-of-band carriers. In You's reserved subcarriers block coding scheme, $\alpha$ is always set to 1 . In conclusion, the 9-APSK out-of-band carrier technique proposed in this section differs from You's technique in three aspects. Firstly, the carriers are located at the outof-band region and using them do not affect the bandwidth efficiency of the system. 


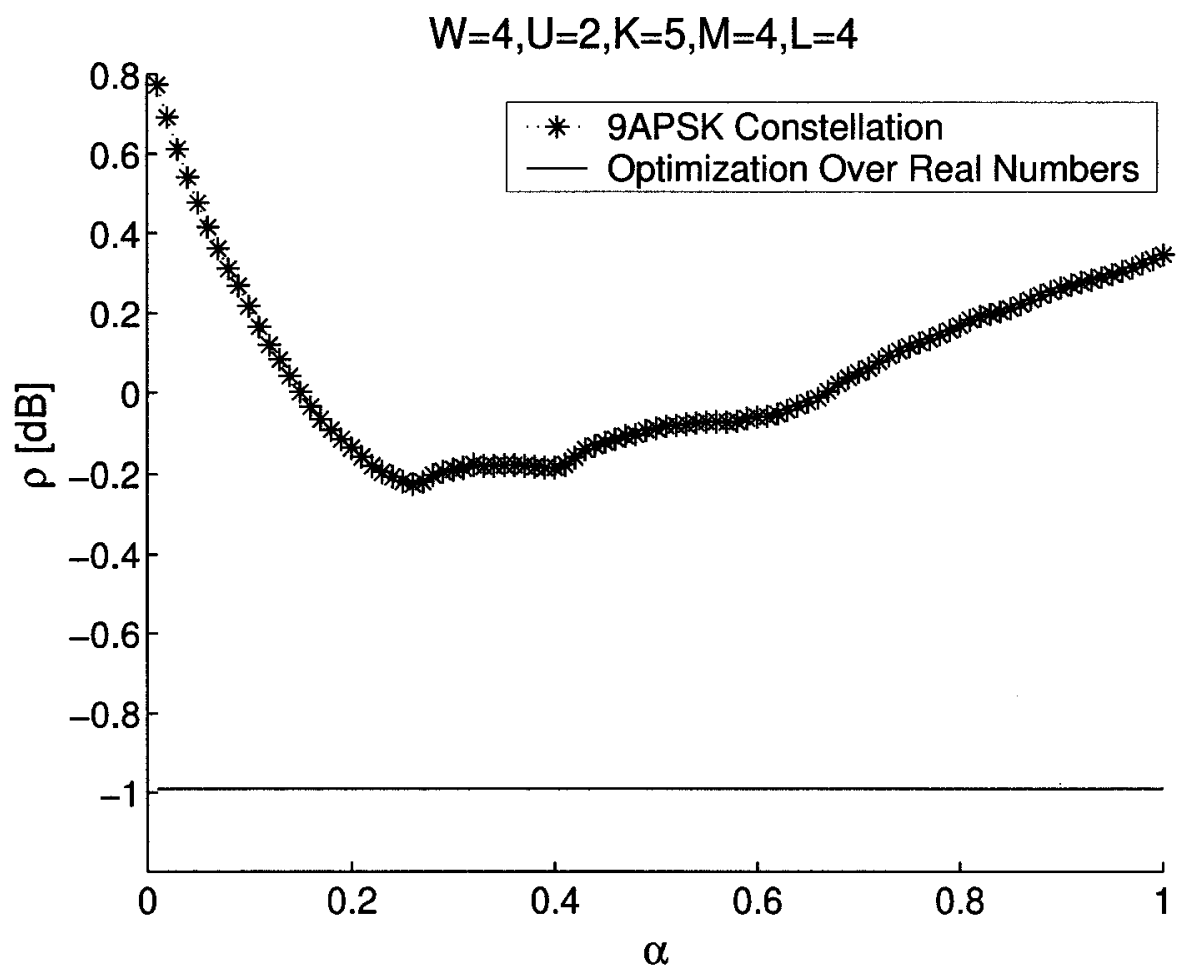

Figure 4.10: Normalized average optical power $(\rho)$ versus scaling factor for TCMSM code with $W=4, U=2, K=5, M=4$ and $L=4$.

Secondly, a scaling factor $\alpha$ is introduced to further reduce the average optical power. Lastly, the out-of-band carriers are chosen from 9-APSK constellation rather than QPSK constellation.

\subsubsection{Performance Analysis}

The performance of scaled 9-APSK out-of-band carrier technique applied to normal MSM systems, tone injection systems and in-band trellis-coded systems are shown in Figures 4.11 to 4.14 . All the systems achieve the same bit rate and the same BER of $10^{-6}$. Normalized average optical power defined in (2.37) and normalized peak optical power defined in (2.38) are plotted versus bandwidth efficiency for $L=1$ and 


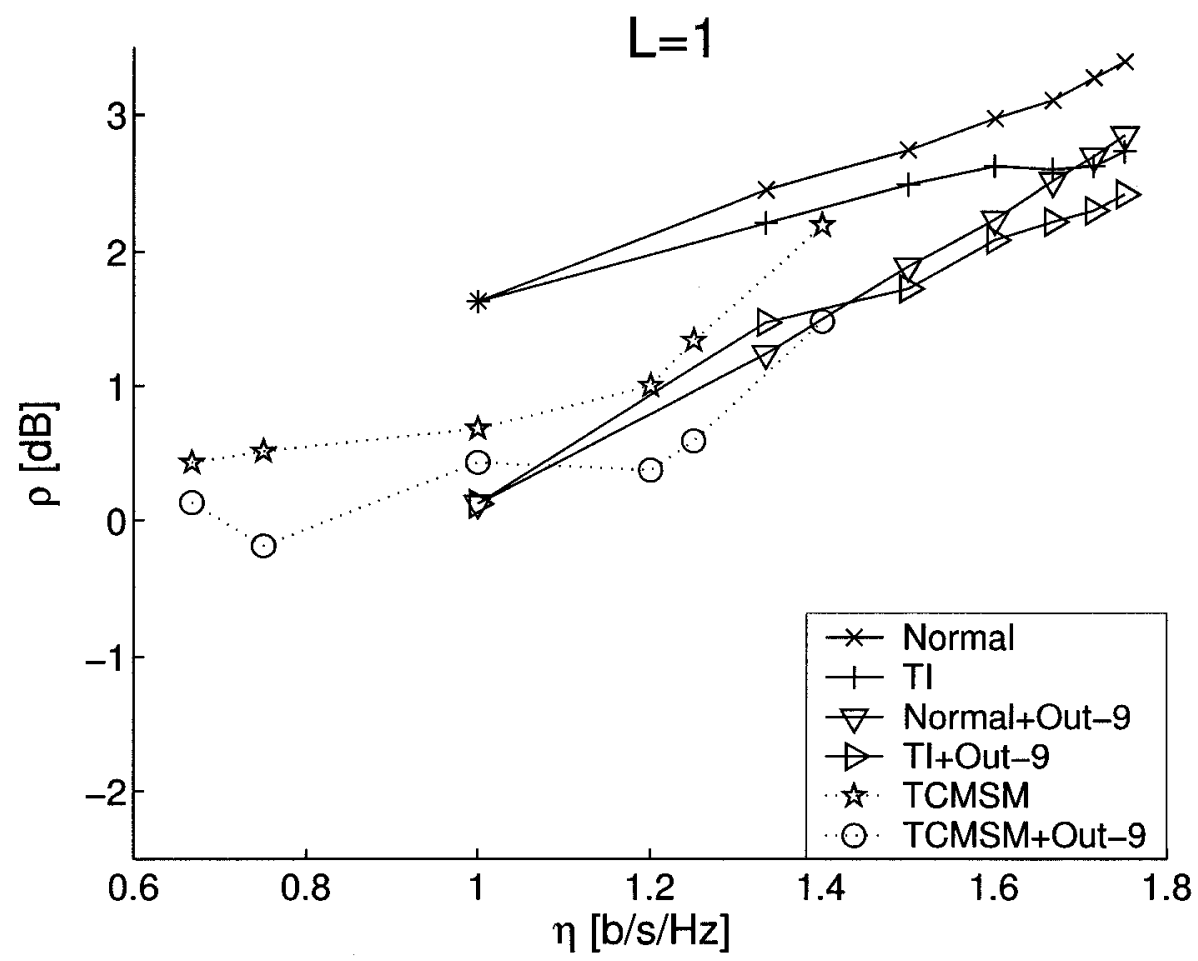

Figure 4.11: Normalized average optical power $(\rho)$ versus bandwidth efficiency $(\eta)$ for scaled 9-APSK $L=1$ out-of-band techniques

$L=4$. The performance using $L=2$ and $L=3$ scaled 9-APSK out-of-band carriers are included in the Appendix.

As is done in Section 4.2.2, we measure the minimum, maximum and average reduction in the average optical power over all bandwidth efficiencies achieved by out-of-band carriers. The minimum, maximum and average increase in the peak optical power is also measured for all bandwidth efficiencies. Note the reductions and increases are calculated by comparing the in-band system applied with out-ofband carriers and the in-band system alone. On average, applying $L=1$ scaled 9APSK out-of-band carrier to normal MSM system achieves $0.43 \mathrm{~dB}$ optical of average optical power reduction with a peak optical power increase of $0.41 \mathrm{~dB}$ optical. When 


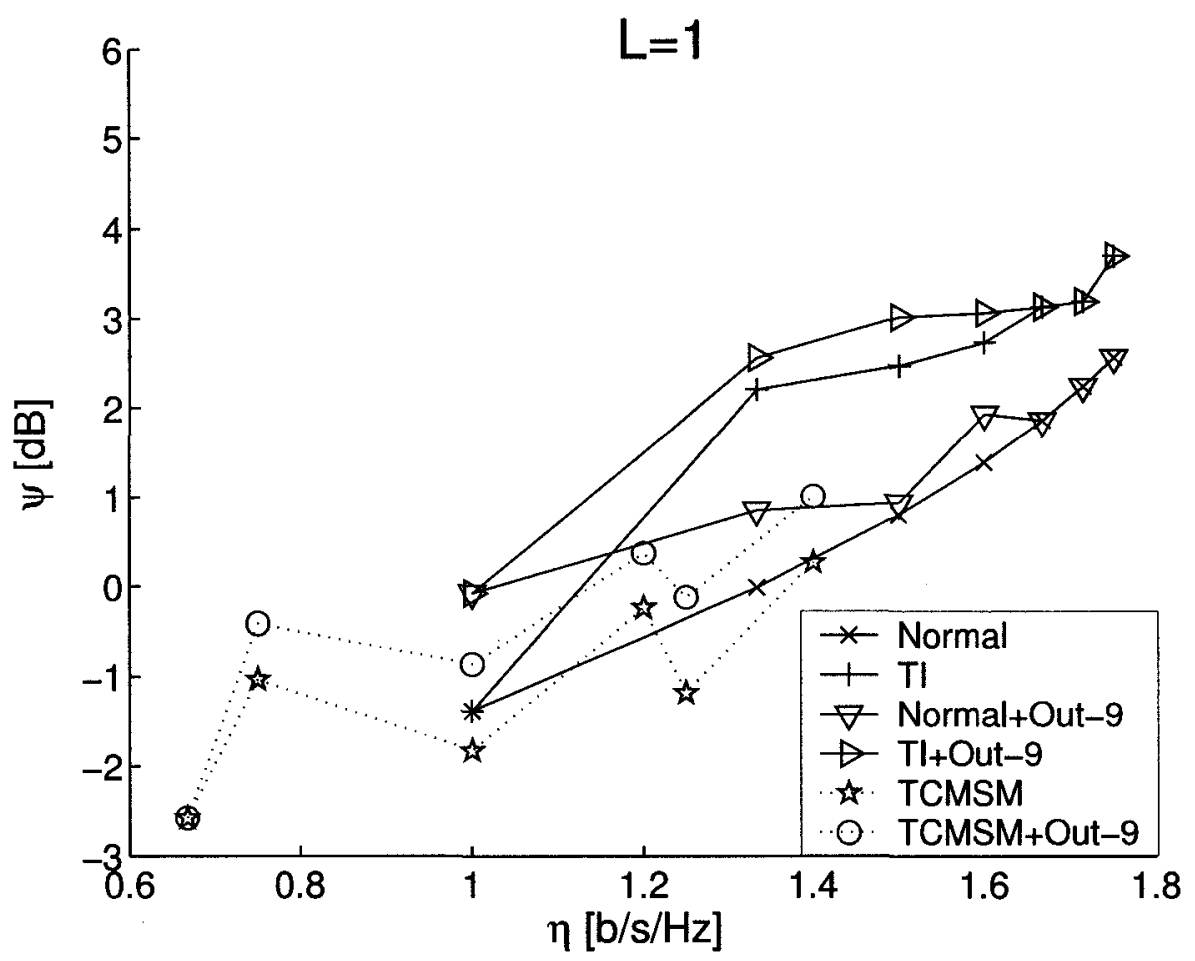

Figure 4.12: Normalized peak optical power $(\psi)$ versus bandwidth efficiency $(\eta)$ for 9-APSK scaled $L=1$ out-of-band techniques 


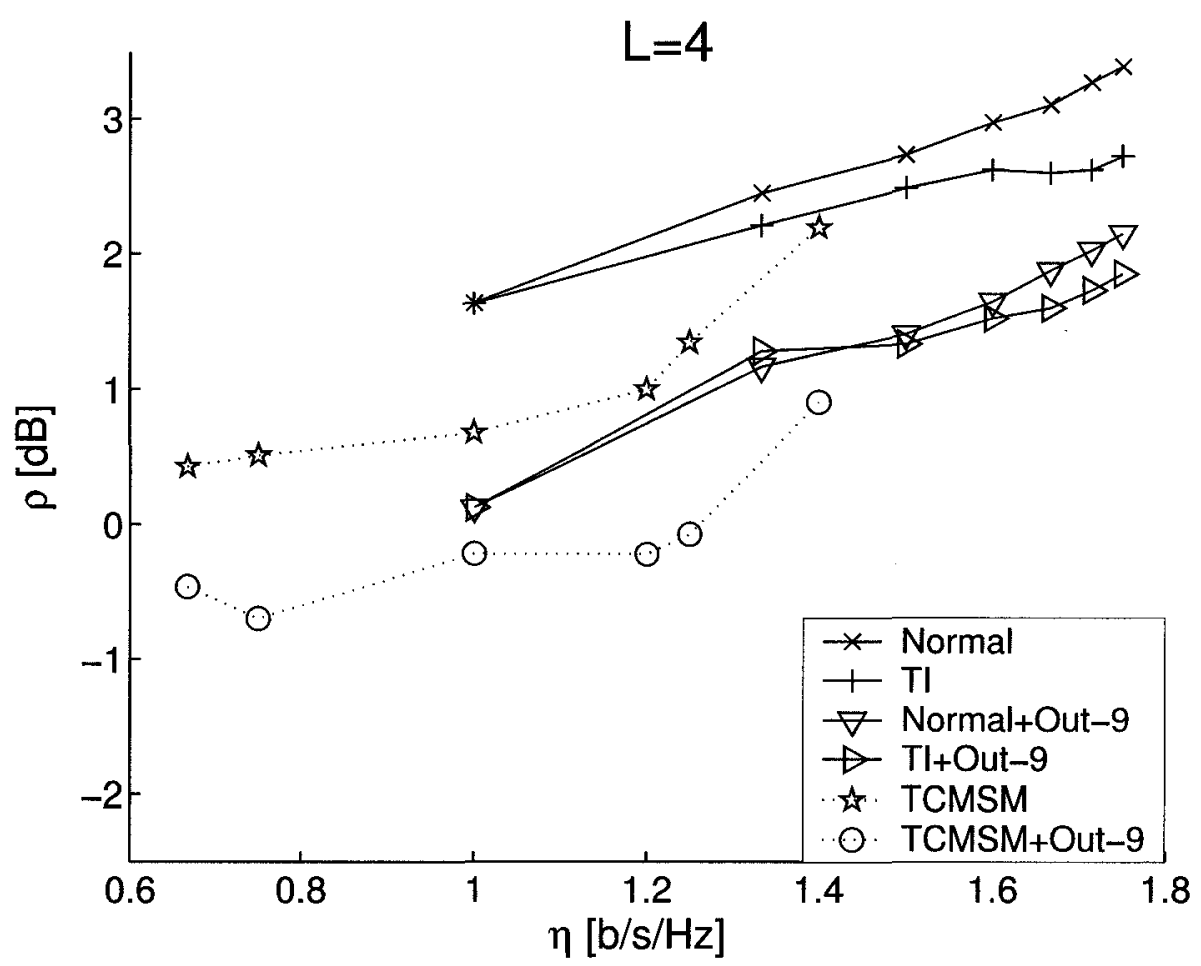

Figure 4.13: Normalized average optical power $(\rho)$ versus bandwidth efficiency $(\eta)$ for scaled 9-APSK $L=4$ out-of-band techniques 


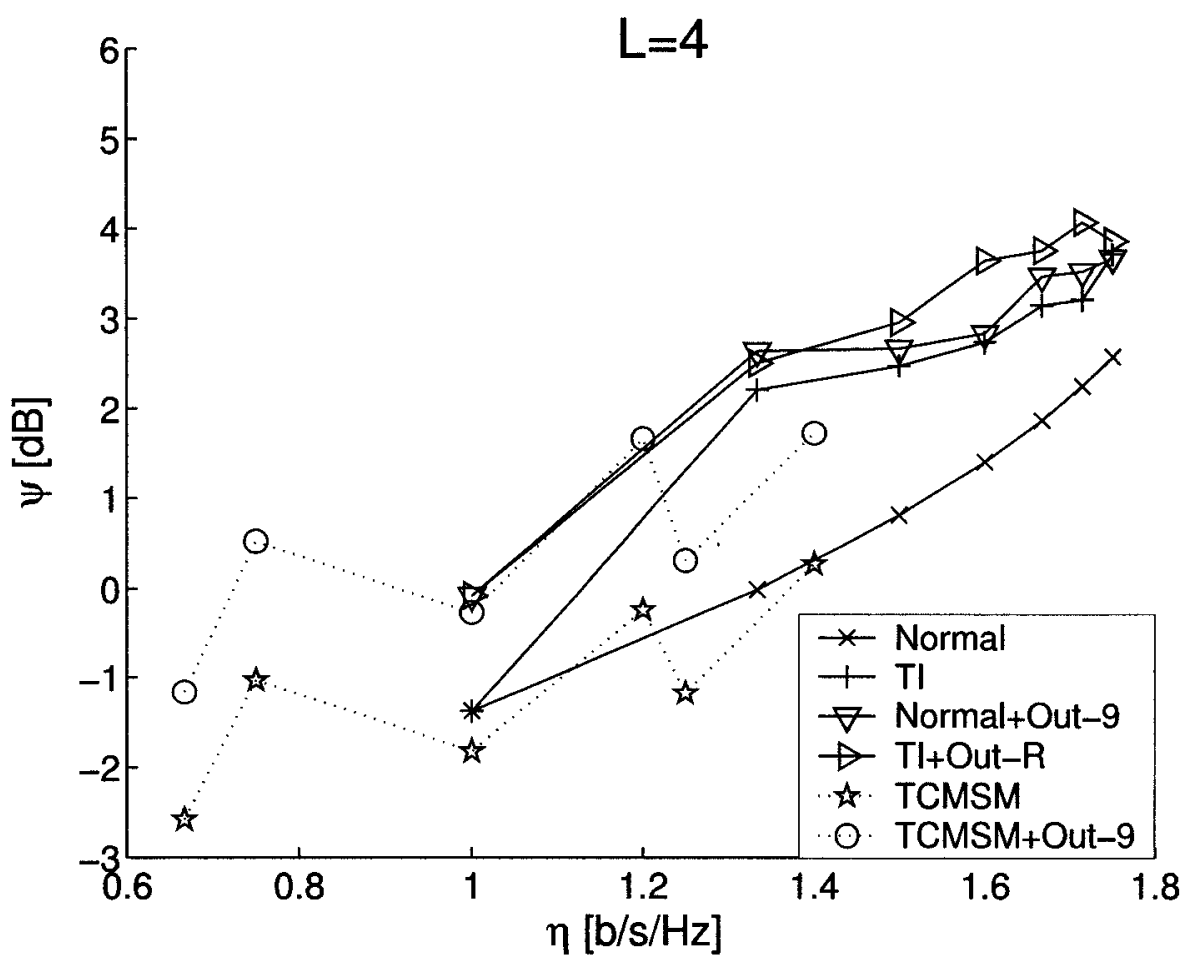

Figure 4.14: Normalized peak optical power $(\psi)$ versus bandwidth efficiency $(\eta)$ for scaled 9-APSK $L=4$ out-of-band techniques 
increasing $L$ to 4 , the average optical power reduction is $1.31 \mathrm{~dB}$ and the peak optical power increase is $1.60 \mathrm{~dB}$. In contrast, applying $L=1$ scaled 9-APSK out-of-band carrier to the tone injection system and best in-band trellis codes achieved an average optical power reductions of $0.65 \mathrm{~dB}$ and $0.56 \mathrm{~dB}$ on average respectively. The increases in the peak optical power are $0.36 \mathrm{~dB}$ and $0.67 \mathrm{~dB}$ on average respectively. When increasing $L$ to 4 , the average optical power reductions are $1.07 \mathrm{~dB}$ and $1.16 \mathrm{~dB}$ on average respectively for tone injection systems and the best in-band trellis codes. The peak optical power increases are $0.66 \mathrm{~dB}$ and $1.56 \mathrm{~dB}$ on average respectively. The average optical power reduction, peak optical power increase as well as the additional memory requirement for applying scaled 9-APSK out-of-band carriers to in-band systems are detailed in Tables 4.4 and 4.5 for $L=1$ and $L=4$ respectively. The performance using $L=2$ and $L=3$ scaled 9-APSK out-of-band carriers are presented in the Appendix and the performance are found to be between those of $L=1$ and $L=4$.

Parallel to the results in Section 4.2, we notice in Figures 4.11 and 4.13 that inband trellis coding with scaled 9-APSK out-of-band carriers perform better at lower bandwidth efficiencies, while normal MSM system with scaled 9-APSK out-of-band carriers perform better at higher bandwidth efficiencies. Again, in-band trellis-coded systems have a complex receiver architecture which requires the use of a Viterbi decoder.

Comparing scaled 9-APSK and real-amplitude out-of-band carrier techniques, we observe that real-amplitude out-of-band carriers achieved greater reduction in average optical power at the expense of increased peak optical power and nearly 8 times of the memory requirement. On average, when applying to normal MSM system, real-amplitude out-of-band carriers has a greater average optical power reduction of $0.83 \mathrm{~dB}$ and a $0.46 \mathrm{~dB}$ higher peak optical power than 9-APSK out-of-band carriers 


\begin{tabular}{|c|c|c|c|c|}
\hline In-band & $\eta$ & Reduction of $\rho[\mathrm{dB}]$ & Increase in $\psi[\mathrm{dB}]$ & Memory [bytes] \\
\hline \multirow[t]{7}{*}{ Normal } & 1.00 & 1.50 & 1.30 & 8 \\
\hline & 1.33 & 1.21 & 0.87 & 20 \\
\hline & 1.50 & 0.85 & 0.14 & 68 \\
\hline & 1.60 & 0.73 & 0.53 & 260 \\
\hline & 1.67 & 0.59 & 0.00 & 1028 \\
\hline & 1.71 & 0.58 & 0.00 & 4100 \\
\hline & 1.75 & 0.54 & 0.00 & 16388 \\
\hline \multirow[t]{7}{*}{ TI } & 1.00 & 1.50 & 1.30 & 8 \\
\hline & 1.33 & 0.74 & 0.36 & 20 \\
\hline & 1.50 & 0.76 & 0.55 & 68 \\
\hline & 1.60 & 0.53 & 0.33 & 260 \\
\hline & 1.67 & 0.38 & 0.00 & 1028 \\
\hline & 1.71 & 0.32 & 0.00 & 4100 \\
\hline & 1.75 & 0.31 & 0.00 & 16388 \\
\hline \multirow[t]{6}{*}{ TCMSM } & 0.67 & 0.30 & 0.00 & 12 \\
\hline & 0.75 & 0.70 & 0.62 & 36 \\
\hline & 1.00 & 0.25 & 0.96 & 132 \\
\hline & 1.20 & 0.63 & 0.61 & 260 \\
\hline & 1.25 & 0.75 & 1.06 & 132 \\
\hline & 1.40 & 0.71 & 0.74 & 516 \\
\hline
\end{tabular}

Table 4.4: Performance of $L=1$ scaled 9-APSK out-of-band carriers applied to normal MSM systems and the best in-band trellis codes 


\begin{tabular}{|c|c|c|c|c|}
\hline In-band & $\eta$ & Reduction of $\rho[\mathrm{dB}]$ & Increase in $\psi[\mathrm{dB}]$ & Memory [bytes] \\
\hline \multirow[t]{7}{*}{ Normal } & 1.00 & 1.50 & 1.30 & 20 \\
\hline & 1.33 & 1.29 & 2.65 & 68 \\
\hline & 1.50 & 1.34 & 1.86 & 260 \\
\hline & 1.60 & 1.33 & 1.43 & 1028 \\
\hline & 1.67 & 1.23 & 1.59 & 4100 \\
\hline & 1.71 & 1.25 & 1.27 & 16388 \\
\hline & 1.75 & 1.24 & 1.09 & 65540 \\
\hline \multirow[t]{7}{*}{ TI } & 1.00 & 1.50 & 1.30 & 20 \\
\hline & 1.33 & 0.93 & 0.30 & 68 \\
\hline & 1.50 & 1.16 & 0.48 & 260 \\
\hline & 1.60 & 1.11 & 0.90 & 1028 \\
\hline & 1.67 & 1.01 & 0.61 & 4100 \\
\hline & 1.71 & 0.90 & 0.87 & 16388 \\
\hline & 1.75 & 0.88 & 0.15 & 65540 \\
\hline \multirow[t]{6}{*}{ TCMSM } & 0.67 & 0.89 & 1.41 & 36 \\
\hline & 0.75 & 1.21 & 1.55 & 132 \\
\hline & 1.00 & 0.90 & 1.55 & 516 \\
\hline & 1.20 & 1.22 & 1.90 & 1028 \\
\hline & 1.25 & 1.41 & 1.49 & 516 \\
\hline & 1.40 & 1.29 & 1.45 & 2052 \\
\hline
\end{tabular}

Table 4.5: Performance of $L=4$ scaled 9-APSK out-of-band carriers applied to normal MSM systems and the best in-band trellis codes 
McMaster University - Dept. Elec. \& Comp. Eng.

with $L=4$. When applying to in-band trellis-coded systems, real-amplitude out-ofband carriers have a greater average optical power reduction of $0.75 \mathrm{~dB}$ and a greater peak optical power of $0.76 \mathrm{~dB}$ than scaled 9-APSK out-of-band carriers with $L=4$.

\subsubsection{Conclusions}

In this section, the in-phase and quadrature amplitudes of the out-of-band carriers are optimized over the 9-APSK constellation. A scaling factor $\alpha$ is introduced over all out-of-band carriers to achieve additional average optical power reduction. Applying $L=4$ out-of-band carriers to in-band systems, an average optical power reduction up to $1.50 \mathrm{~dB}$ is achieved with an increase in the peak optical power as high as $2.65 \mathrm{~dB}$. The additional memory requirement ranges from 8 bytes to 65540 bytes depending on the in-band system and $L$.

Although the average optical power reductions achieved by scaled 9-APSK out-ofband carriers are smaller than those achieved by real-amplitude out-of-band carriers, the increase in peak optical powers as well as the additional memory requirements are reduced. Thus, the scaled 9-APSK out-of-band carrier technique is more suitable for applications where the complexity of the transmitter must be limited.

\subsection{Out-of-band techniques with reduced memory requirements}

The use of out-of-band carriers with both real-numbered and discrete-constellation amplitudes do not increase receiver complexity. However, additional memory is required at the transmitter. In applications where the transmitter complexity is also strictly limited and little additional memory could be implemented, the out-of-band 
techniques proposed in previous sections may become infeasible to employ. To address this problem, in this section we propose out-of-band techniques with reduced memory requirement. This technique is suitable for systems which can tolerate very little additional complexity. The trade-off between memory requirement and average optical power reduction capabilities is presented.

Firstly, notice that according to (4.4) there are $4^{W}$ possible input symbols in a normal MSM system with $W$ in-band carriers. The distribution of the average optical power requirements for these symbols is worth investigating. If there are a small number of symbols with very high average optical power requirement, then designing out-of-band carriers only for these symbols may reduce the overall system average optical power while significantly reducing the memory requirement.

Figure 4.15 shows the distribution of average optical powers for all input symbols in a normal MSM system with $W=4,5,6,7$. It is observed that a small portion of the input symbols have high average optical power requirement. This suggests that we can apply out-of-band carriers to part of but not all of the input symbols with the highest average optical power requirement. This will trade average optical power reduction for reduced memory requirement.

Figures 4.16 and 4.17 plot the performance of a normal MSM system with $L=4$ out-of-band carriers applied to the worst (highest average optical power) $25 \%, 50 \%$ and $75 \%$ of the input symbols. The performance of applying out-of-band carriers to all input symbols is also shown for comparison. All systems achieve the same bit rate and the same BER of $10^{-6}$. Note the symbols chosen to be applied with out-of-band carriers are the ones with the highest average optical power requirements, and they are always applied with $L=4$ out-of-band carriers. It is clear from the figures that there is a trade-off between memory requirement and average optical power reduction capabilities. Moreover, the percentage of loss in average optical power reduction and the percentage of savings in memory requirements are roughly the same. This can 

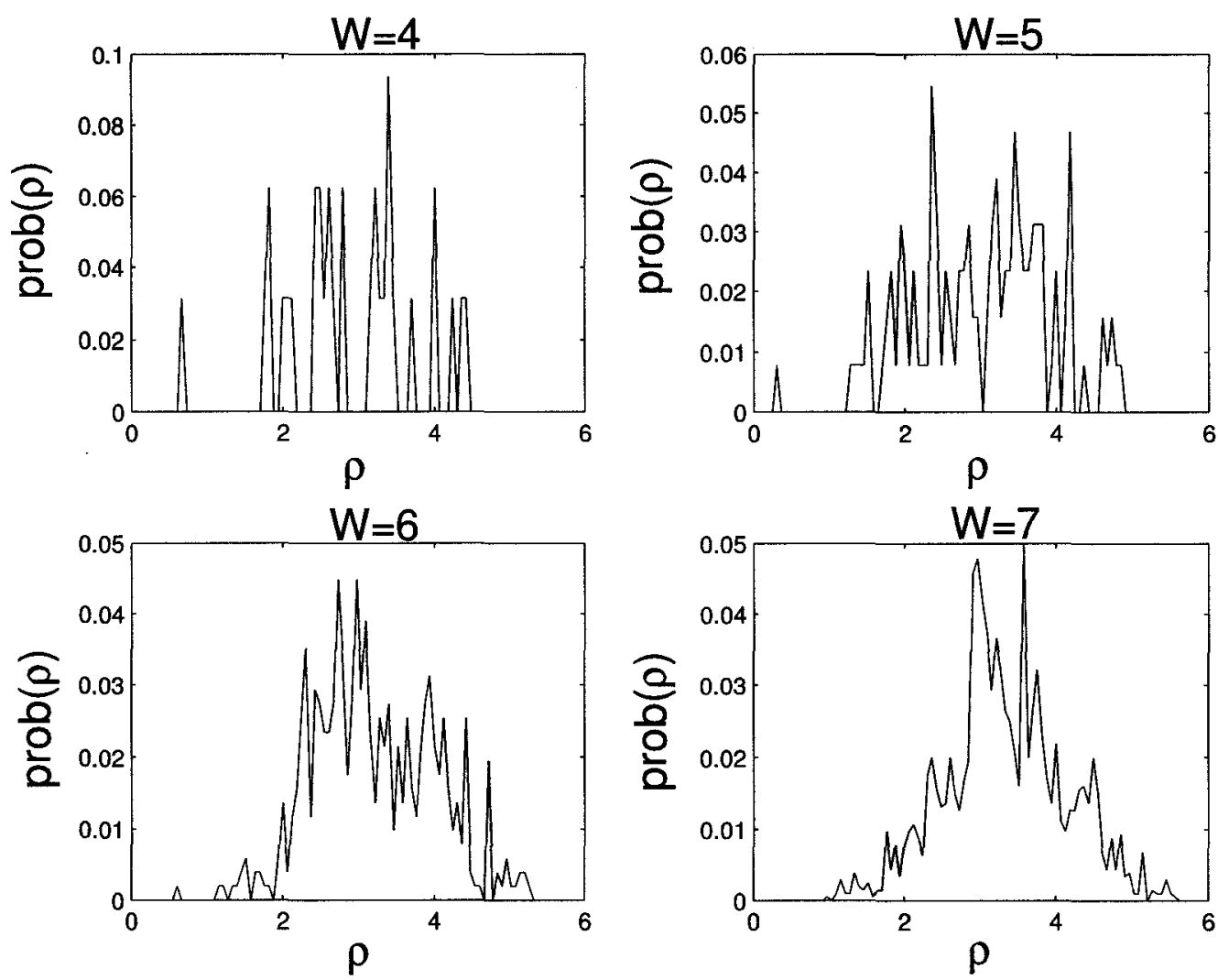

Figure 4.15: Probability distribution of normalized average optical power for all input symbols in normal MSM systems 


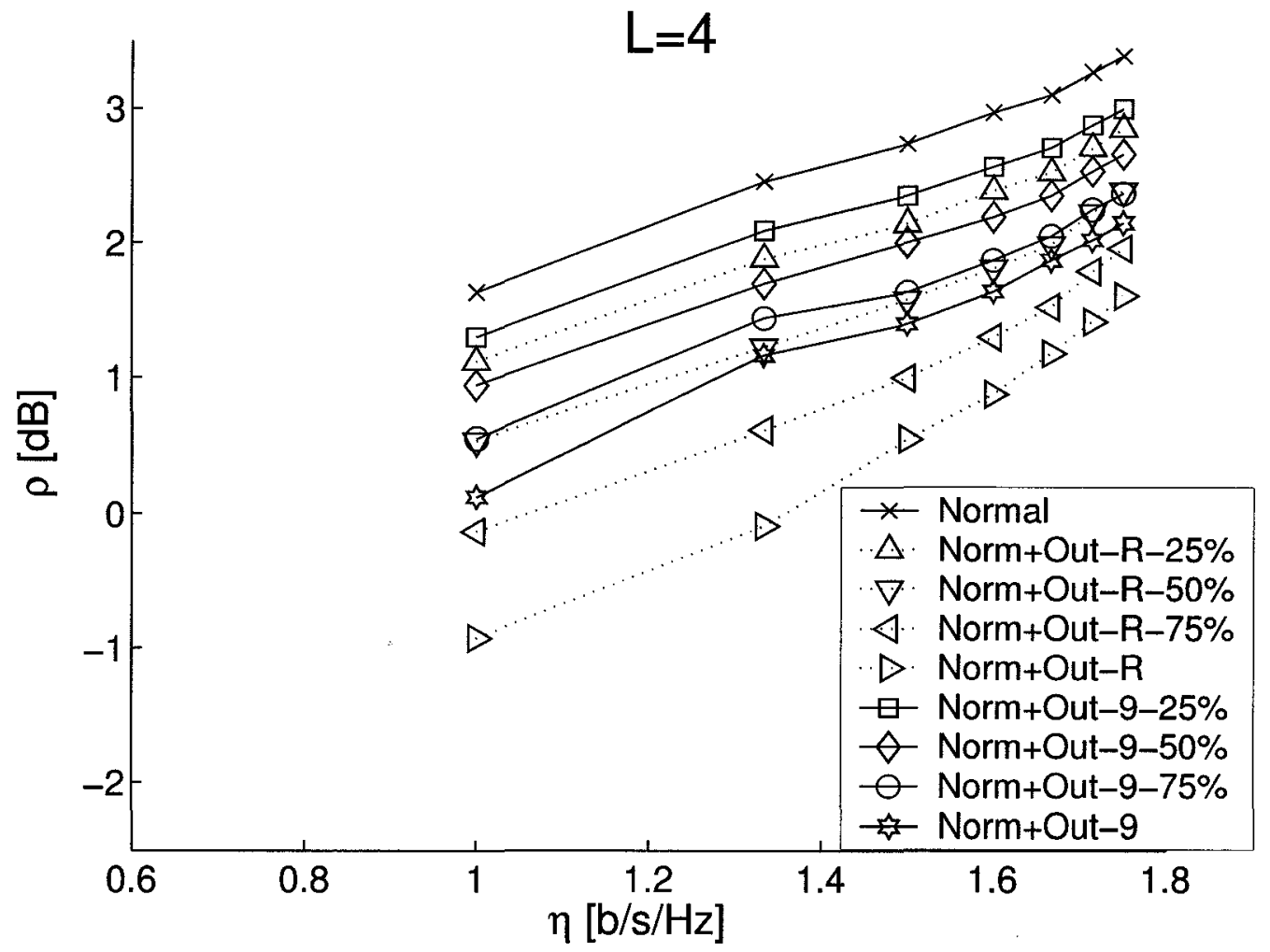

Figure 4.16: Normalized average optical power $(\rho)$ versus bandwidth efficiency $(\eta)$ for reduced memory $L=4$ out-of-band techniques

help to predict the achievable average optical power reduction when using any amount of memory. The example shown here use normal MSM system and $L=4$ out-of-band carriers for illustration purpose. However, application of this technique to other inband systems with any number of out-of-band carriers is staightforward.

In conclusion, the use of reduced-memory out-of-band carrier technique is proposed in this section. This technique trades average optical power reduction capabilities with memory requirement and is suitable for applications where complexity of the transmitter and receiver must be strictly limited. 


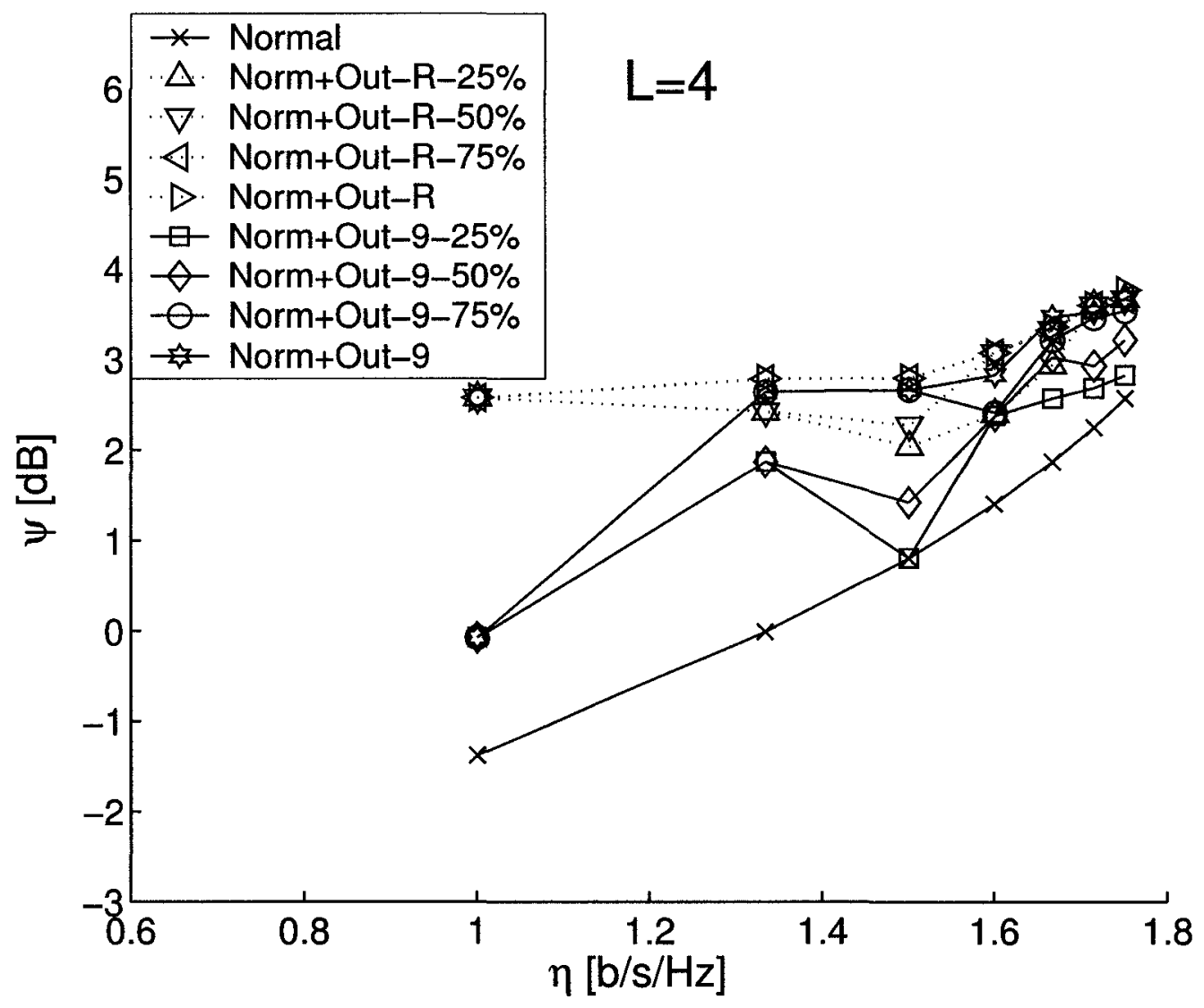

Figure 4.17: Normalized peak optical power $(\psi)$ versus bandwidth efficiency $(\eta)$ for reduced memory $L=4$ out-of-band techniques 


\subsection{Out-of-band carriers applied to trellis-coded systems with DC detection technique}

In this section, the in-band trellis-coded system with DC detection technique proposed in Section 3.5 is applied with real-amplitude and scaled 9-APSK out-of-band carriers. The performance with $L=4$ out-of-band carriers are shown in Figures 4.18 and 4.19 and the results are listed in Table 4.6. All systems achieve the same bit rate and the same BER of $10^{-6}$. On average, an average optical power reduction of $2.08 \mathrm{~dB}$ is achieved with a simultaneous peak power increase of $2.16 \mathrm{~dB}$ by applying DC detection technique with $L=4$ real-amplitude out-of-band carriers to the best inband trellis codes. The average reduction is $1.32 \mathrm{~dB}$ for the average optical power and the average increase is $1.40 \mathrm{~dB}$ for the peak optical power with $L=4$ scaled 9-APSK out-of-band carriers and DC detection technique.

Note the resulting systems employ all three proposed techniques for average optical power reduction. As a result, such systems achieve the best reduction in average optical power. The peak optical power is increased, however, this increase is smaller than that of using the out-of-band carriers without in-band trellis codes or DC detection technique. This is because both in-band trellis codes and DC detection technique help to reduce the peak optical power. The complexity of this system is the greatest of all systems proposed, which may limit its potential application.

\subsection{Conclusions}

In this chapter, out-of-band carrier design techniques are introduced to reduce the average optical power in MSM wireless optical systems. The use of out-of-band carriers is possible in wireless optical communications due to the inherent isolation of optical signals. By containing the optical signals in a room, out-of-band carriers signals do 


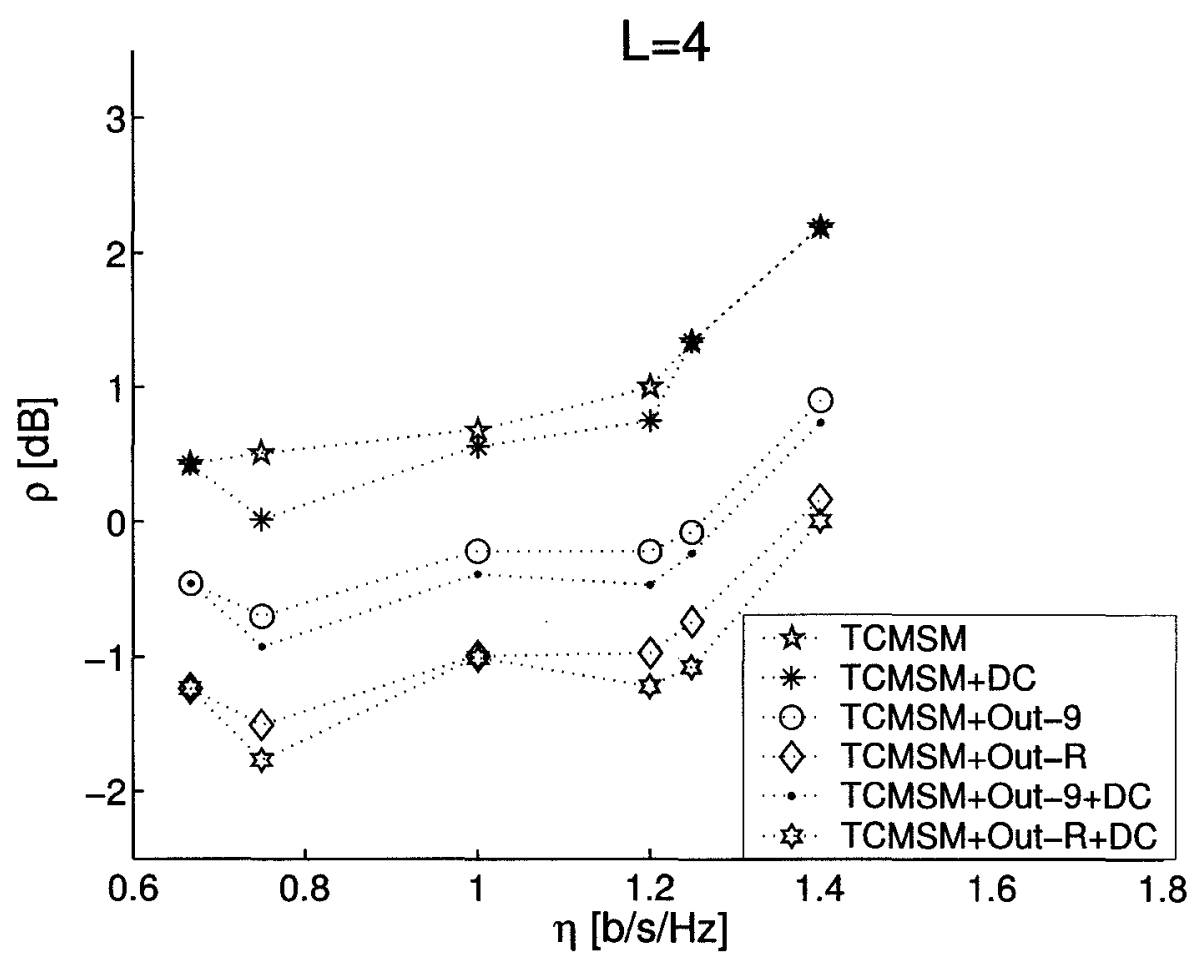

Figure 4.18: Normalized average optical power $(\rho)$ versus bandwidth efficiency $(\eta)$ for out-of-band carriers with DC detection technique 


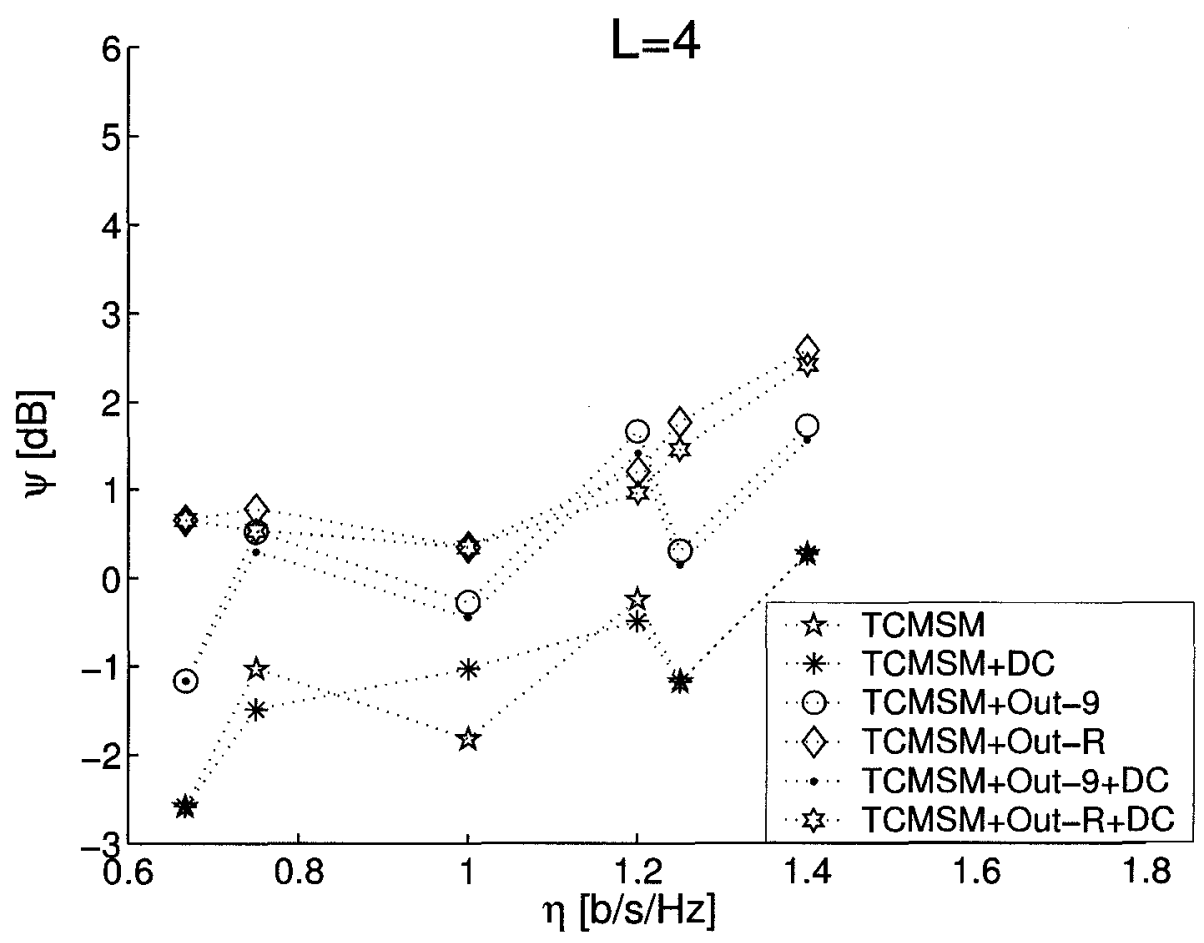

Figure 4.19: Normalized peak optical power $(\psi)$ versus bandwidth efficiency $(\eta)$ for out-of-band carriers with DC detection technique 


\begin{tabular}{|l|l|l|l|}
\hline$\eta$ & Out-of-band amplitudes & Reduction of $\rho[\mathrm{dB}]$ & Increase in $\psi[\mathrm{dB}]$ \\
\hline \multirow{3}{*}{0.67} & 9 -APSK & 0.89 & 1.41 \\
\cline { 2 - 4 } & Real & 1.66 & 3.23 \\
\hline \multirow{2}{*}{0.75} & 9-APSK & 1.44 & 1.33 \\
\cline { 2 - 4 } & Real & 2.28 & 1.57 \\
\hline \multirow{2}{*}{1.00} & 9-APSK & 1.07 & 1.38 \\
\cline { 2 - 4 } & Real & 1.69 & 2.18 \\
\hline \multirow{3}{*}{1.20} & 9 -APSK & 1.47 & 1.66 \\
\cline { 2 - 4 } & Real & 2.22 & 1.20 \\
\hline \multirow{2}{*}{1.25} & $9-$ APSK & 1.57 & 1.33 \\
\cline { 2 - 4 } & Real & 2.41 & 2.64 \\
\hline \multirow{2}{*}{1.40} & $9-$ APSK & 1.45 & 1.29 \\
\cline { 2 - 4 } & Real & 2.19 & 2.15 \\
\hline
\end{tabular}

Table 4.6: Performance of DC detection technique with $L=4$ out-of-band carriers applied to the best in-band trellis codes 
not interfere with other ongoing communications schemes. In contrast, in RF systems the spectrum is strictly limited. Moreover, out-of-band signals will cause co-channel interference with other communication systems. Therefore use of out-of-band carriers is not feasible in RF systems.

The out-of-band carriers in wireless optical systems do not carry information, but are designed with the purpose of reducing the average optical power. They are filtered out by the channel and the receiver, therefore the bandwidth efficiency of the system is unaffected. The in-phase and quadrature amplitudes on out-of-band carriers are designed using either an optimization algorithm over real numbers or an exhaustive search over scaled 9-APSK constellation. As a result, significant average optical power reduction is achieved. The reduction in average optical power is as high as $2.56 \mathrm{~dB}$ optical. Such techniques operate well at high bandwidth efficiencies when applied to normal MSM systems and tone injection systems. In contrast, when applied to in-band trellis coding systems, the resulting system operates well at low bandwidth efficiencies.

Unlike trellis-coded systems where a Viterbi decoder is required at the receiver, the use of out-of-band carriers does not greatly complicate the receiver implementation since each data bin can be detected independently. This is important since the receivers are typically mobile terminals whose power consumption and complexity are tightly limited. The additional complexity of out-of-band technique is introduced at the transmitter. Circuits used to generate out-of-band carriers must be implemented, and additional memory in the form of a lookup table is required. Although scaled 9-APSK out-of-band carriers achieve a smaller average optical power reduction than real-amplitude out-of-band carriers, they require only about $1 / 8$ of the memory for real-amplitude out-of-band carriers. Moreover, out-of-band carriers can be designed for selected input symbols with high average optical power to further reduce memory requirement. Such techniques trade average optical power reduction capabilities for 
memory requirements.

Apart from the increased complexity at the transmitter, another cost of out-ofband technique is an increase in the peak optical power. Real-amplitude out-of-band carriers have a larger peak optical power increase than scaled 9-APSK out-of-band carriers. Therefore, out-of-band techniques are useful for a low-complexity system whose constraint on the peak optical power is not tight.

By combining out-of-band technique with in-band trellis coding and DC detection techniques, the best reduction in average optical power is achieved. The increase in the peak optical power is also reduced compared to using out-of-band technique alone. The system employing all three techniques achieved $2.63 \mathrm{~dB}$ of average optical power reduction with $1.73 \mathrm{~dB}$ of peak optical power increase at $\eta=1$ compared to a normal MSM system. However, this system has the most complexity which may restrict its applications.

Conventionally, the average optical power $\rho$ and peak optical power $\psi$ are plotted versus the number of in-band carriers, $W$. Such plots are presented in Figures 4.20 and 4.21 for completeness and the average optical power reduction is as high as $4.50 \mathrm{~dB}$ optical. However, as explained in Section 3.4, the plot of $\rho$ and $\psi$ versus bandwidth efficiency $\eta$ more clearly justifies the performance of various schemes versus their cost and is therefore a better comparison. 


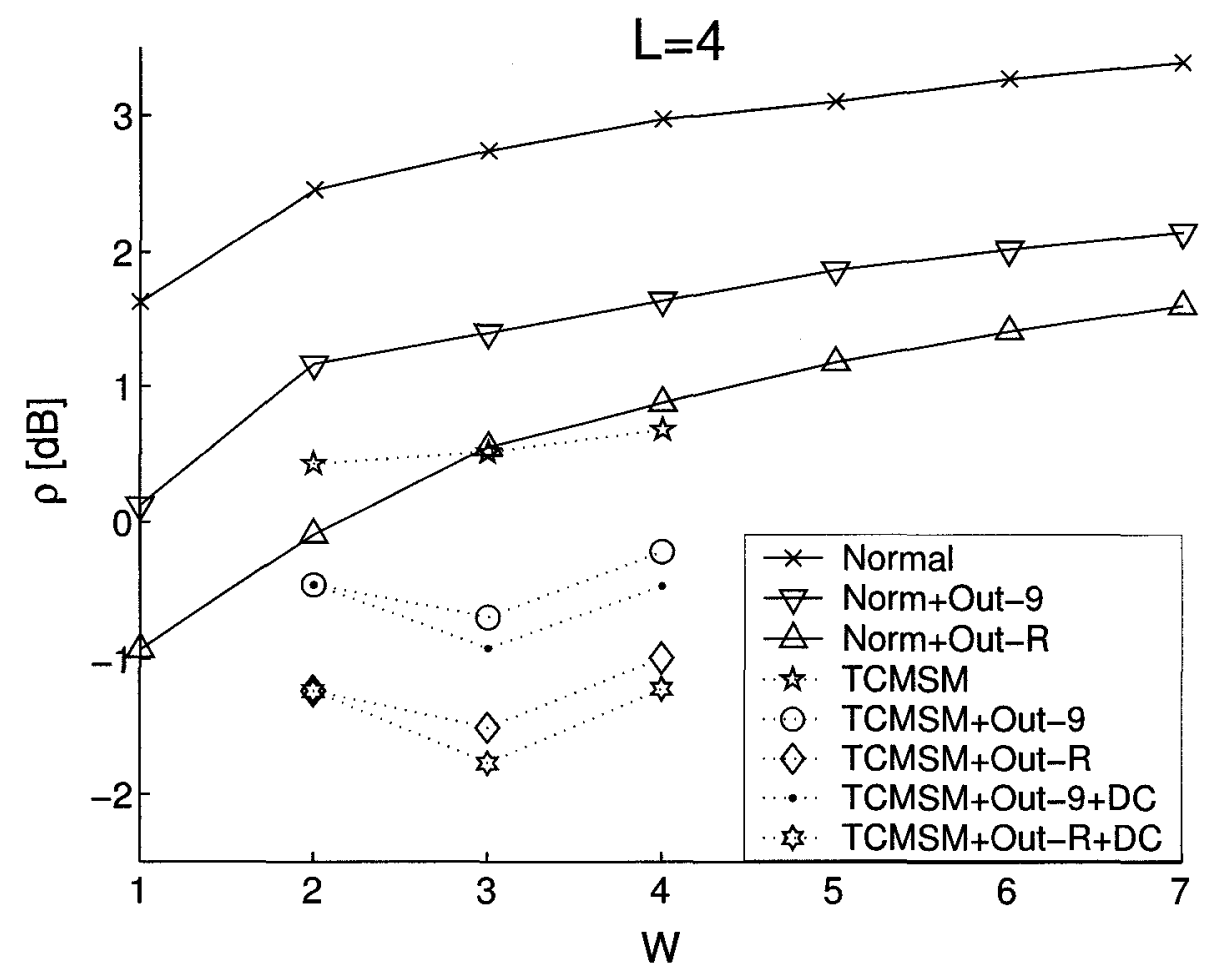

Figure 4.20: Normalized average optical power $(\rho)$ versus number of in-band carriers $(W)$ for out-of-band techniques 


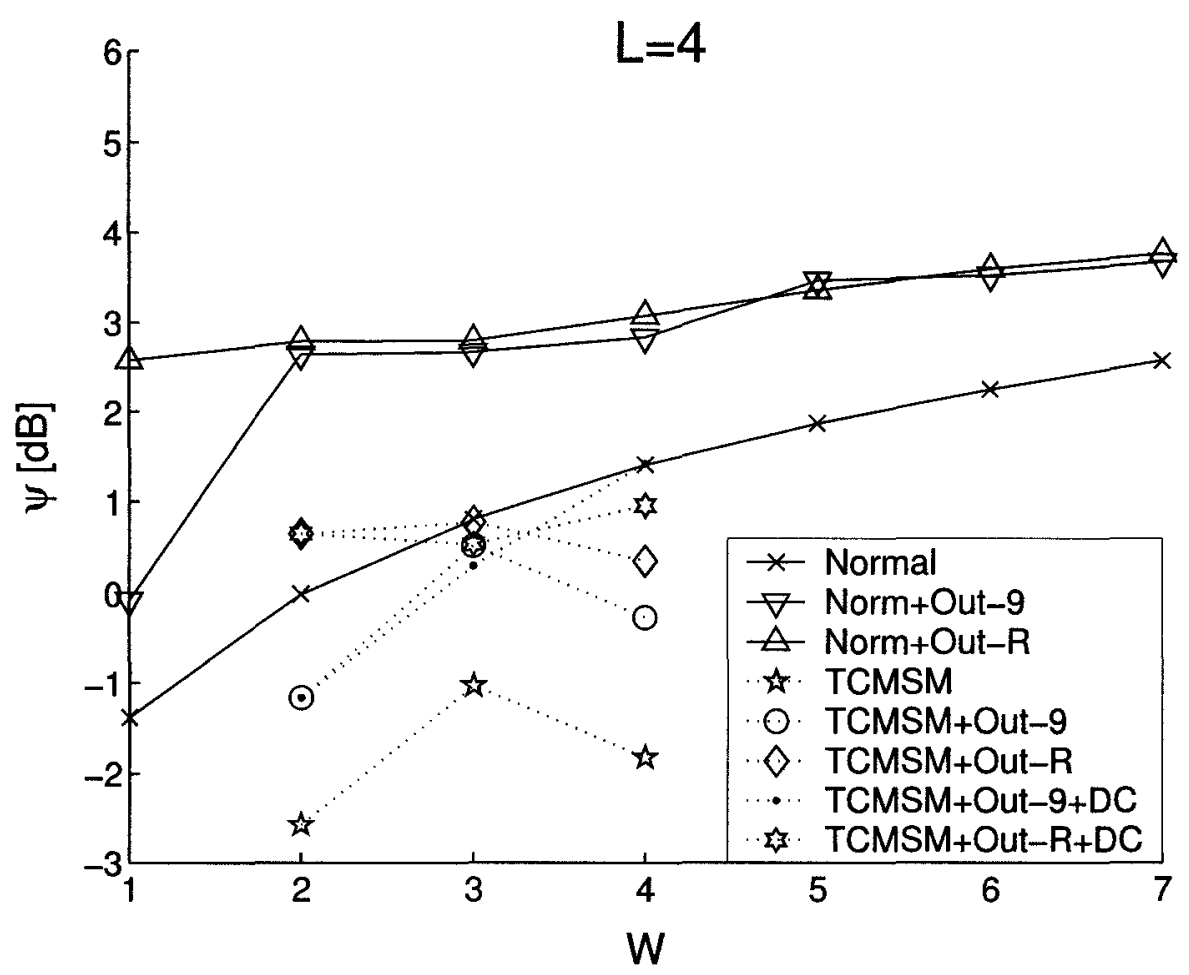

Figure 4.21: Normalized peak optical power $(\psi)$ versus number of in-band carriers $(W)$ for out-of-band techniques 


\section{Chapter 5}

\section{Discussions and Conclusions}

Diffuse wireless optical links provide a low-cost alternative solution for short-range high-rate data communications other than RF communications. Optical transmitter and receiver devices for this application are available in high volume at low cost. Moreover, the infrared spectrum region is unregulated worldwide, further reducing the system cost. However, multiple reflections of the transmitted signals cause high multipath distortion which reduce the optical power efficiency of such systems.

Multiple-subcarrier modulated wireless optical systems employ multiple narrowband carriers to convey information. Such systems use the bandwidth efficiently by allocating the narrow-band carriers at frequencies where the channel attenuation and the noise power are low. For frequencies where the noise power is high such as the lower frequency spectrum region where fluorescent light noise power is concentrated, no carriers are designed. The MSM wireless optical systems also have the capability to support multiple-access by sending independent information on different carriers.

Due to safety constraints, wireless optical systems have limited average optical power. Because intensity modulation is used in these systems, the average optical power is proportional to the bias level added to ensure the non-negativity of the electrical signal. Therefore, the addition of many sinusoids in MSM wireless optical 
systems results in a high average optical power requirement. This thesis address the problem of average optical power reduction in MSM wireless optical systems.

A similar problem arises in electrical MSM systems, where the addition of many sinusoids leads to a high PAPR. Electrical PAPR reduction have been studied extensively and many methods have been proposed. However, electrical PAPR reduction focus on the envelope of the squared amplitude of the waveform. In contrast, average optical power reduction reduce the negative peak amplitude for each data symbol. Due to this difference in the objective function, direct application of electrical PAPR reduction techniques in wireless optical systems is not guaranteed to achieve good average optical power reduction. In fact, a well-known electrical PAPR reduction technique named tone injection has been applied directly in wireless optical systems in Chapter 3. Only moderate reductions in average optical power up to $0.66 \mathrm{~dB}$ have been achieved. Therefore, to effectively reduce the average optical power of MSM wireless optical systems, novel techniques need to be developed.

To address this problem, we have developed three techniques: in-band trelliscoding, out-of-band carrier design and DC detection. These techniques outperformed existing average optical power reduction methods. The combination of these techniques yield an average optical power reduction up to $2.63 \mathrm{~dB}$, which is equal to $5.26 \mathrm{~dB}$ of electrical power reduction.

In-band trellis coding techniques apply the conventionally used trellis-coded modulation to MSM wireless optical systems. Constellation expansion is done by adding a zero-amplitude point in all frequency carrier constellations. Assuming either a 2-state or 4-state trellis, a sub-optimal search algorithm is developed and the algorithm is run in both constrained and unconstrained way to find the codes which best reduces the average optical power. In this manner, average optical power is reduced on the order of $1 \mathrm{~dB}$ optical and the peak optical power is nearly unaffected. It is noted that this technique operates well at low bandwidth efficiencies. 
Out-of-band carrier design technique uses the huge bandwidth available in wireless optical communications due to the inherent isolation of the optical signals. The outof-band carriers do not send information, but are designed to reduce the average optical power. They are filtered out by the channel and the receiver and not used in detection. The carrier amplitudes are designed either by optimization over realvalued in-phase and quadrature amplitudes or by an exhaustive search over a discrete constellation. Out-of-band carrier design technique offers significant average optical power reductions on the order of $2 \mathrm{~dB}$ optical at higher bandwidth efficiencies. The gains are achieved with no additional complexity at the receiver and a lookup table at the transmitter. The size of the lookup table is quantified and varies between 20 bytes to 524288 bytes with 4 out-of-band carriers. For applications where the transmitter complexity is tightly constrained, reduced-memory technique is proposed and only part of the input symbols with high average optical power are applied with out-of-band carriers. The drawback of out-of-band techniques is that the peak optical power is increased.

The DC detection technique includes the symbol-by-symbol bias signal as another dimension in the signal space. The receiver detects the symbol-by-symbol bias to improve the receiver BER performance. By applying DC detection to a in-band coding with out-of-band carrier system, additional average optical power reduction on the order of $0.5 \mathrm{~dB}$ optical is achieved.

In Section 5.1, we compare the performance of the three proposed techniques by applying them to the same baseline normal system. Gains achieved by either applying each technique individually or applying combinations of the three techniques are quantified. Moreover, the advantages and disadvantages of each technique is analyzed, along with possible application scenarios. A design guide for choosing techniques for average optical power reduction in MSM wireless optical systems is given based on system constraints, including system complexity, noise characteristics and peak 


\begin{tabular}{|l|l|l|}
\hline $\begin{array}{l}\text { Scheme } \\
\text { Employed }\end{array}$ & $\begin{array}{l}\text { Reduction } \\
\text { in } \rho[\mathrm{dB}]\end{array}$ & $\begin{array}{l}\text { Reduction } \\
\text { in } \psi[\mathrm{dB}]\end{array}$ \\
\hline TCMSM & 0.95 & 0.44 \\
\hline TCMSM and DC & 1.07 & -0.35 \\
\hline Out-9 & 1.50 & -1.30 \\
\hline Out-R & 2.56 & -3.96 \\
\hline TCMSM and Out-9 & 1.85 & -1.11 \\
\hline TCMSM and Out-R & 2.62 & -1.73 \\
\hline TCMSM and DC and Out-9 & 2.02 & -0.94 \\
\hline TCMSM and DC and Out-R & 2.63 & -1.73 \\
\hline
\end{tabular}

Table 5.1: Reduction in $\rho$ and $\psi$ by applying various technique to a baseline normal MSM system at $\eta=1$

optical power budget. Some directions for future work are given in Section 5.2.

\subsection{Discussions on average optical power reduc- tion techniques}

The normal MSM system operating at $\eta=1$ is taken as the baseline system. This system have one in-band carrier with a QPSK modulation scheme. The reduction of average optical power and peak optical power by using each technique and combinations of the three technique are reported in Table 5.1, and the average optical power reductions are plotted in Figure 5.1. The legends used are the same as in Table 4.1.

The best in-band trellis code at $\eta=1$ has $W=4$ in-band carriers, while $U=2$ of them are forced to have zero amplitudes as shown in Table 3.5 in Chapter 3. By 


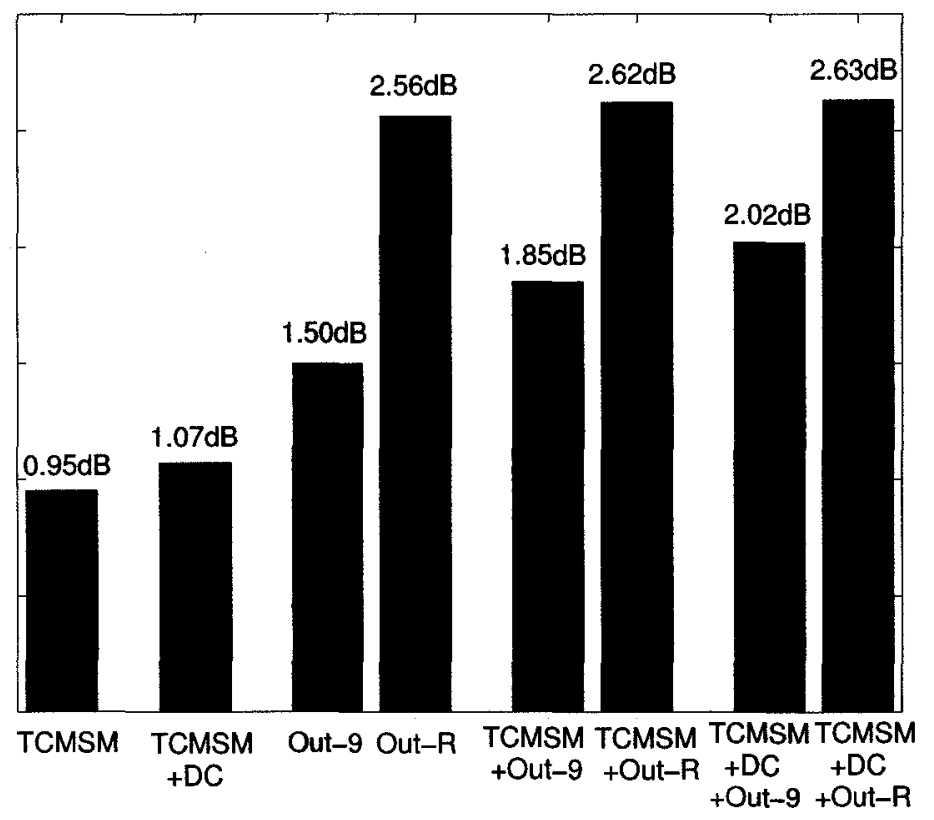

Figure 5.1: Reduction in $\rho$ by applying various technique to a baseline normal MSM system at $\eta=1$

using in-band trellis coding, an average optical power reduction of $0.95 \mathrm{~dB}$ is achieved with a simultaneous peak optical power reduction of $0.44 \mathrm{~dB}$. The advantage of this system is the reduction of the average optical power while leaving the peak optical power nearly unaffected. It should be noted that at some bandwidth efficiencies the peak optical powers are reduced, while at others the peak optical powers are slightly increased. However, the overall peak optical power of in-band coding system is nearly unaffected. The cost of in-band trellis coding technique is the requirement of a trellis encoder at the transmitter and a Viterbi decoder at the receiver. Therefore this technique is useful in applications where both average and peak optical powers are limited and the increase in the system complexity can be tolerated.

The DC detection technique offers reduction in the average optical power, and the reduction in the peak optical power is also possible. Since the baseline $W=1$ normal system have the same DC bias level for all its QPSK symbols, DC detection cannot 
improve its performance. As discussed in Chapter 3, DC detection is applied to a trellis code and the resulting system yields $1.07 \mathrm{~dB}$ of average optical power reduction and $0.35 \mathrm{~dB}$ increase of peak optical power compared to the baseline system.

Although the DC detection technique achieves a smaller average optical power reduction, the additional complexity requirement is another matched filter and associated decision devices, which is relatively low compared to in-band trellis-coded systems. Therefore, it can be implemented with trellis-coded systems to offer average optical power reduction at small cost. One thing to notice is that the fluorescent light sources often introduce strong noise power in low frequencies near DC. In such cases, the performance of DC detection technique will be degraded.

Applying $L=4$ scaled 9-APSK out-of-band carriers to the baseline system yields an average optical power reduction of $1.50 \mathrm{~dB}$ with a $1.30 \mathrm{~dB}$ increase in peak optical power, while $L=4$ real-amplitude out-of-band carriers achieve $2.56 \mathrm{~dB}$ of average optical power reduction with $3.96 \mathrm{~dB}$ of peak optical power increase, all of which are reported in Tables 4.5 and 4.3.

Out-of-band carriers are able to offer more average optical power reduction than in-band trellis coding and DC detection techniques at the cost of increased peak optical power. Moreover, its implementation is simpler than trellis-coded systems. Little additional complexity is required at the receiver and the additional complexity at the transmitter is a lookup table to store out-of-band amplitudes. As reported in Chapter 4 , the size of the lookup table varies from 8 bytes to 524288 bytes, depending on the in-band scheme employed and the number and constellation of out-of-band carriers. When the lookup table size must be limited, out-of-band carriers can be designed for part of the input symbols with high average optical power. It is shown that the percentage of loss in average optical power reduction and the percentage of savings in memory requirements are roughly the same. Therefore, out-of-band technique is the first choice for average optical power reduction in MSM wireless 
optical systems if an increase in the peak optical power can be tolerated. It is useful in applications where complexity of the system must be limited and the constraint on the peak optical power is not tight.

Finally, out-of-band carriers can be applied to the trellis-coded system, or to the trellis-coded system with DC detection technique. Notice such systems employ more than one technique for average optical power reduction, therefore more average optical power reduction is expected. Moreover, the peak optical power increase is smaller than using out-of-band technique alone since in-band trellis coding and DC detection techniques help to reduce the peak optical power. The best reduction is achieved by employing all the three proposed techniques, which is $2.63 \mathrm{~dB}$ optical with $L=4$ real-amplitude out-of-band carriers. The associated cost of this system is that all the additional requirements of the three techniques must be met.

In conclusion, different average optical power reduction techniques should be chosen according to the constraints in wireless optical systems. It can be observed in Figure 5.1 that nearly all the reduction in average optical power is achieved by outof-band carrier design technique. Therefore, if the peak optical power of the system is not tightly constrained, it is highly desirable to employ out-of-band carrier design technique. Additional complexity is added at the transmitter in the form of a lookup table, and the size of the lookup table can be further reduced at the cost of reduced average optical power reduction capabilities. Therefore out-of-band carrier technique is the most promising technique for average optical power reduction in MSM wireless optical systems.

However, if a tight constraint is imposed on the peak optical power but the use of a Viterbi decoder at the receiver can be allowed, it is recommended to use in-band coding technique. The use of DC detection technique is also recommended when the fluorescent light is not strong. Finally, if both additional receiver complexity and an increase in the peak optical power can be tolerated, all the three techniques can be 
McMaster University - Dept. Elec. \& Comp. Eng.

employed to achieve the best reduction in the average optical power.

\section{$5.2 \quad$ Future directions}

In the simulations of this work, it is assumed that the channel is flat at low-frequency in-band region and the noise is white over all in-band carriers. However, the techniques must be modified under non-flat in-band channel or non-white noise. For in-band frequencies where channel attenuation and noise power are high, out-of-band carriers may be designed. Moreover, different in-band carriers may be assigned different transmit powers depending on the SNR at each carrier frequency. To examine the performance of the modified techniques, typical indoor wireless optical channel models can be used.

The design of in-band trellis codes and the design of out-of-band carriers are independent from each other when both techniques are employed. As we observe from Table 5.1 and Figure 5.1, the average optical power reduction of using both inband trellis coding and out-of-band carrier design techniques is not equal to the sum of reductions using either one of them. This suggests that jointly designing in-band trellis codes and out-of-band carriers may yield additional reduction in the average optical power.

The peak optical power is not included in any design objectives. As a result, its performance is not easily predicted and the peak optical power increase can also be very high. Therefore, the peak optical power may be combined with the average optical power in the design objective. A good objective function needs to be developed to achieve high average optical reduction together with a small increase or possibly a reduction in the peak optical power.

In-band trellis-coded systems achieve reduction in average optical power at the cost of a complicated receiver. The receiver need to employ a Viterbi decoder and 
the decoding complexity increases as the number of in-band carriers and bandwidth efficiency increases. Therefore sub-optimal decoders and modified coding schemes are needed to address the complexity issue.

In conclusion, this thesis address the problem of average optical power reduction for diffuse MSM wireless optical systems. The techniques are designed specifically for the constraints in wireless optical MSM systems, which are not present in electrical MSM systems. Significant average optical power reduction up to $2.63 \mathrm{~dB}$ optical is achieved which exceeds previous work. However, extension of these techniques for more general application scenarios need to be developed. Improvement of average optical power efficiency of existing systems can also be expected by jointly optimizing the design of more than one technique. The work presented in this thesis justifies further study into average optical power reduction techniques for indoor MSM wireless optical systems. 


\section{Appendix A}

\section{Performance of using $L=2$ and $L=3$ out-of-band carriers}

As is done in Sections 4.2.2 and 4.3.2 of Chapter 4, the out-of-band carriers with either real-numbered or scaled 9-APSK in-phase and quadrature amplitudes are applied to in-band systems. All systems achieve the same bit rate and the same BER of $10^{-6}$. Figures A.1 to A.4 show the performance using $L=2$ and $L=3$ real-numbered out-of-band carriers, while Figures A.5 to A.8 show the performance with $L=2$ and $L=3$ scaled 9-APSK out-of-band carriers. Normalized average optical power $\rho$ defined in (2.37) and normalized peak optical power $\psi$ defined in (2.38) are plotted versus bandwidth efficiency $\eta$, defined in (2.30). The legends used in figures of this section are consistent with Table 4.1. The impact of these techniques on the average and peak optical powers, as well as the required lookup table size are detailed in Tables A.1, A.2, A.3 and A.4. Note the reductions and increases and calculated by comparing the in-band system applied with out-of-band carriers and the in-band system alone.

To characterize the average optical power reduction and peak optical power increase, we measure the average reduction and the average increase over all bandwidth 


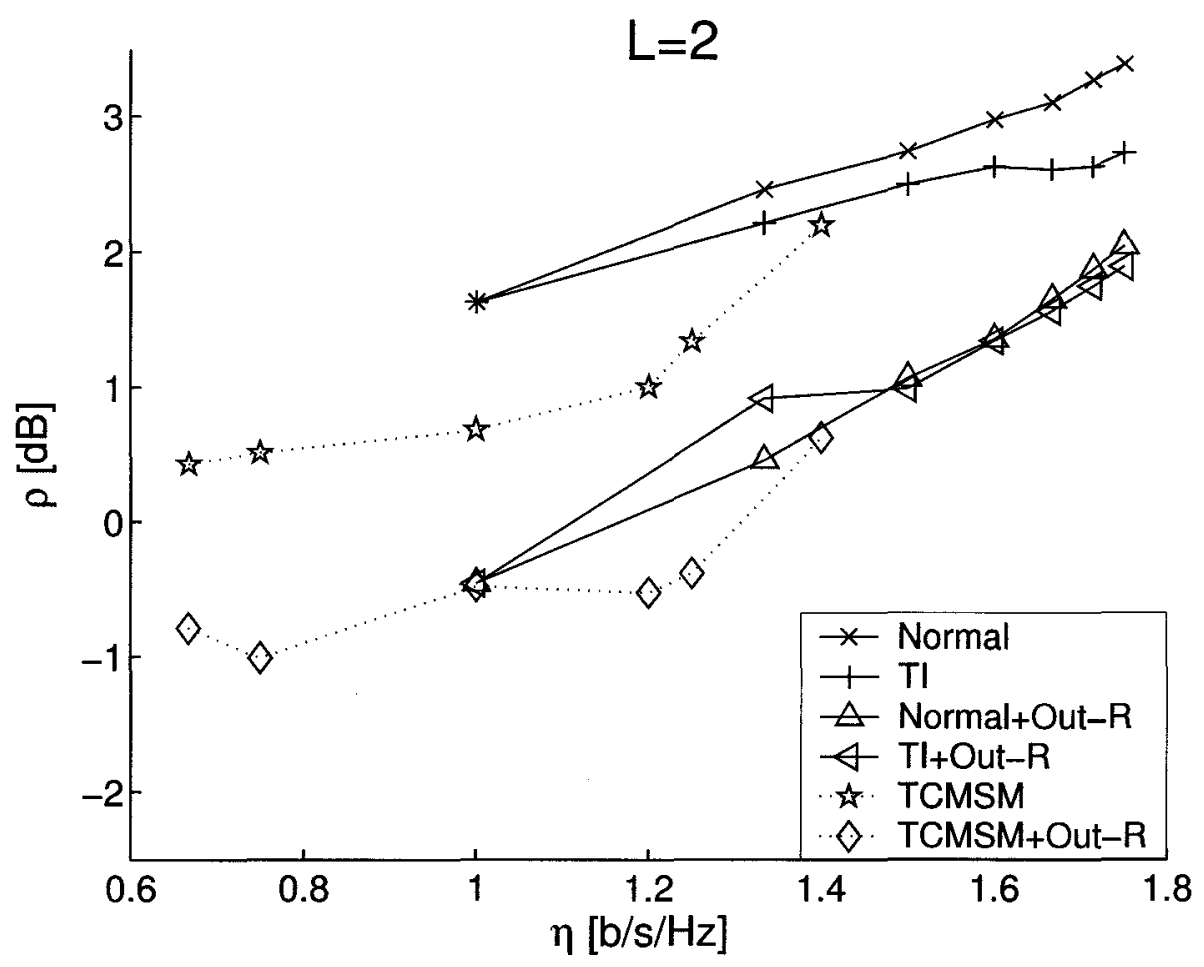

Figure A.1: Normalized average optical power $(\rho)$ versus bandwidth efficiency $(\eta)$ for real-amplitude $L=2$ out-of-band techniques 


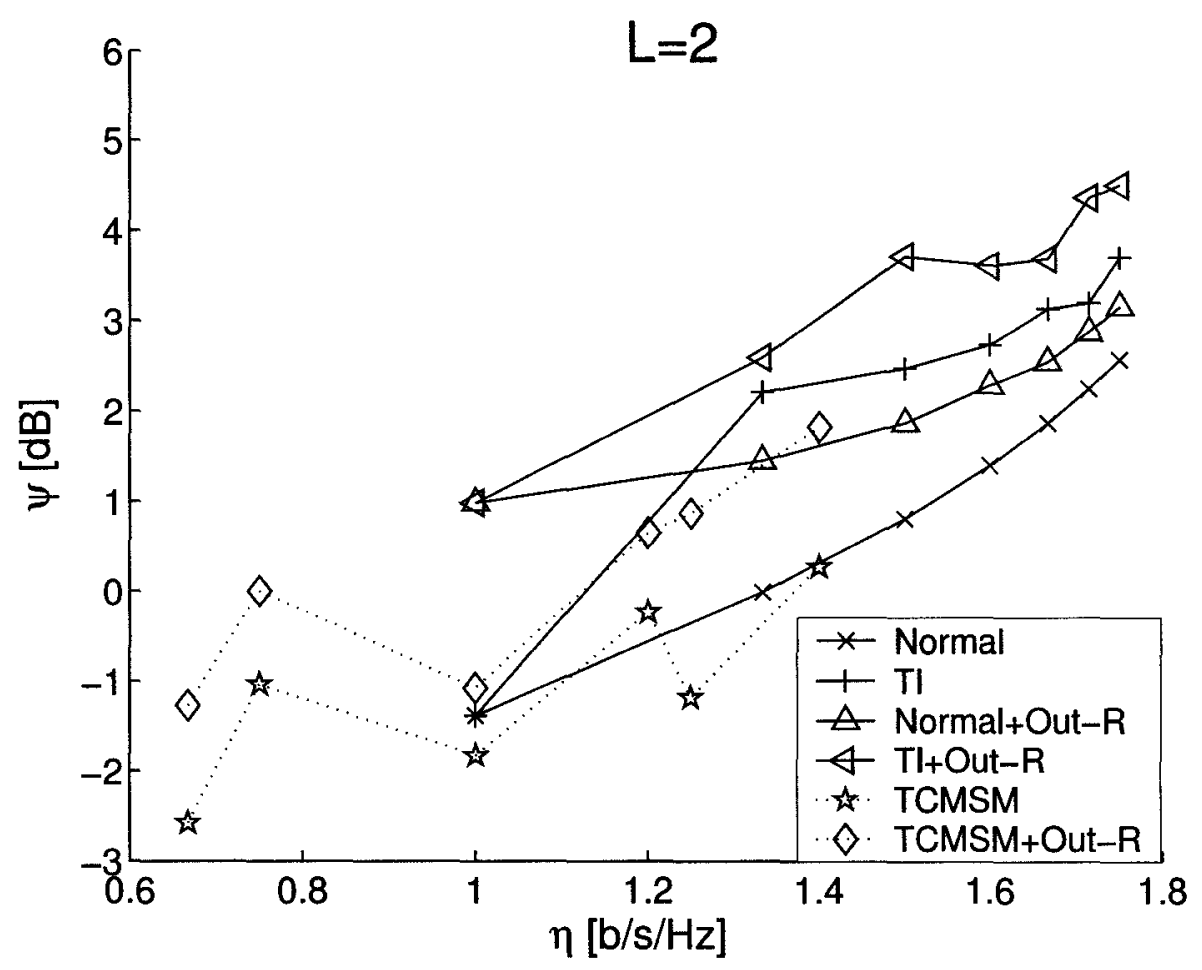

Figure A.2: Normalized peak optical power $(\psi)$ versus bandwidth efficiency $(\eta)$ for real-amplitude $L=2$ out-of-band techniques 


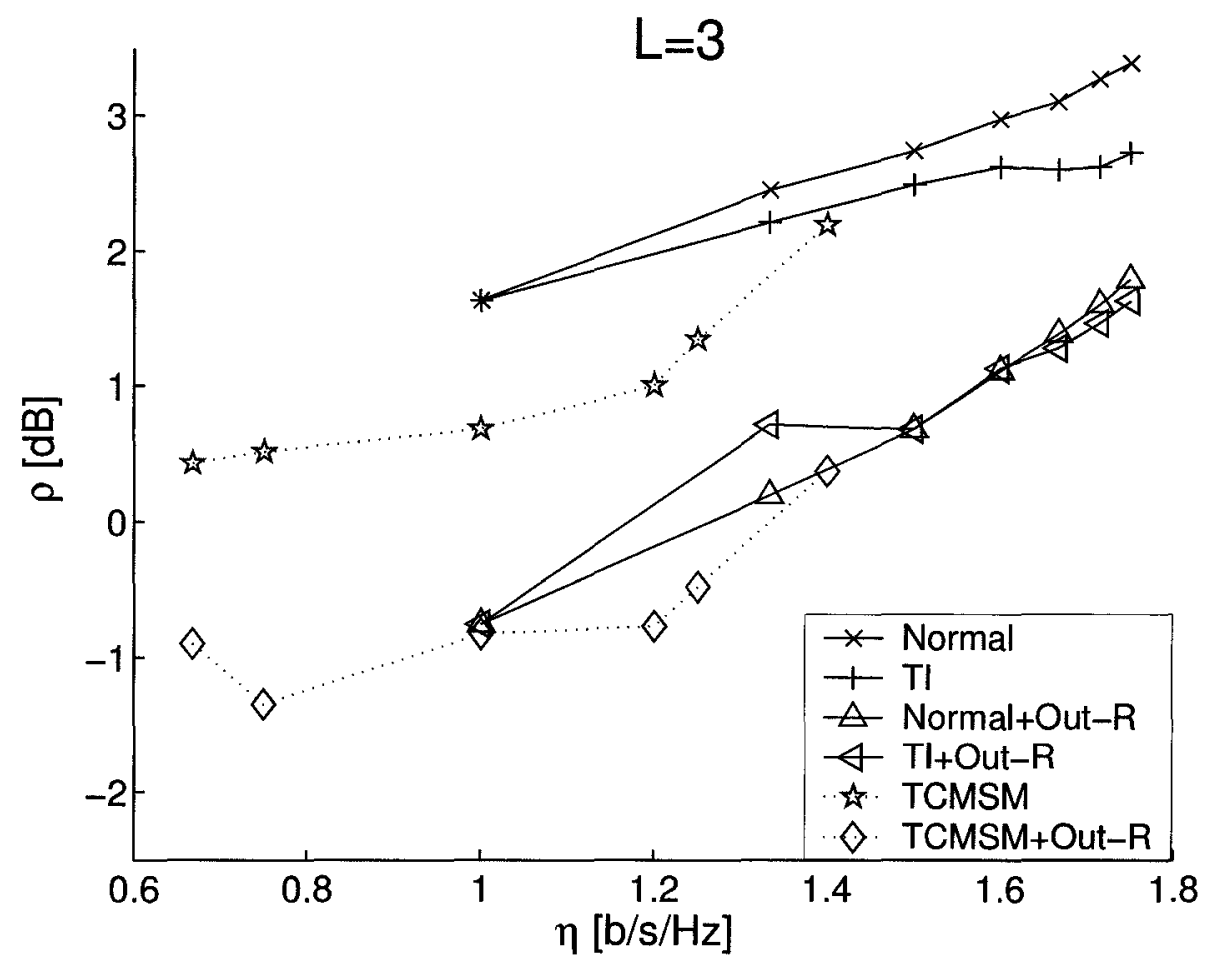

Figure A.3: Normalized average optical power $(\rho)$ versus bandwidth efficiency $(\eta)$ for real-amplitude $L=3$ out-of-band techniques 


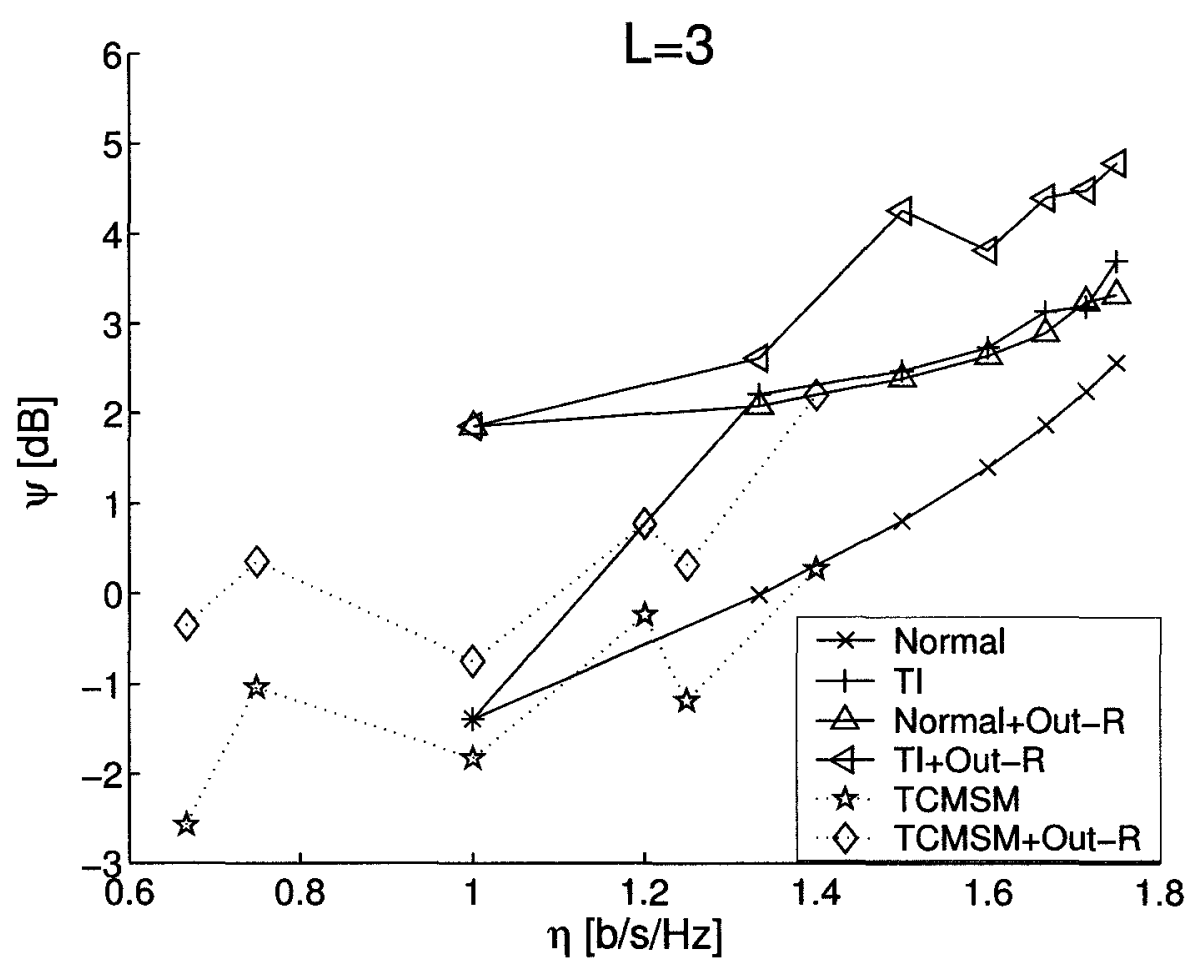

Figure A.4: Normalized peak optical power $(\psi)$ versus bandwidth efficiency $(\eta)$ for real-amplitude $L=3$ out-of-band techniques 


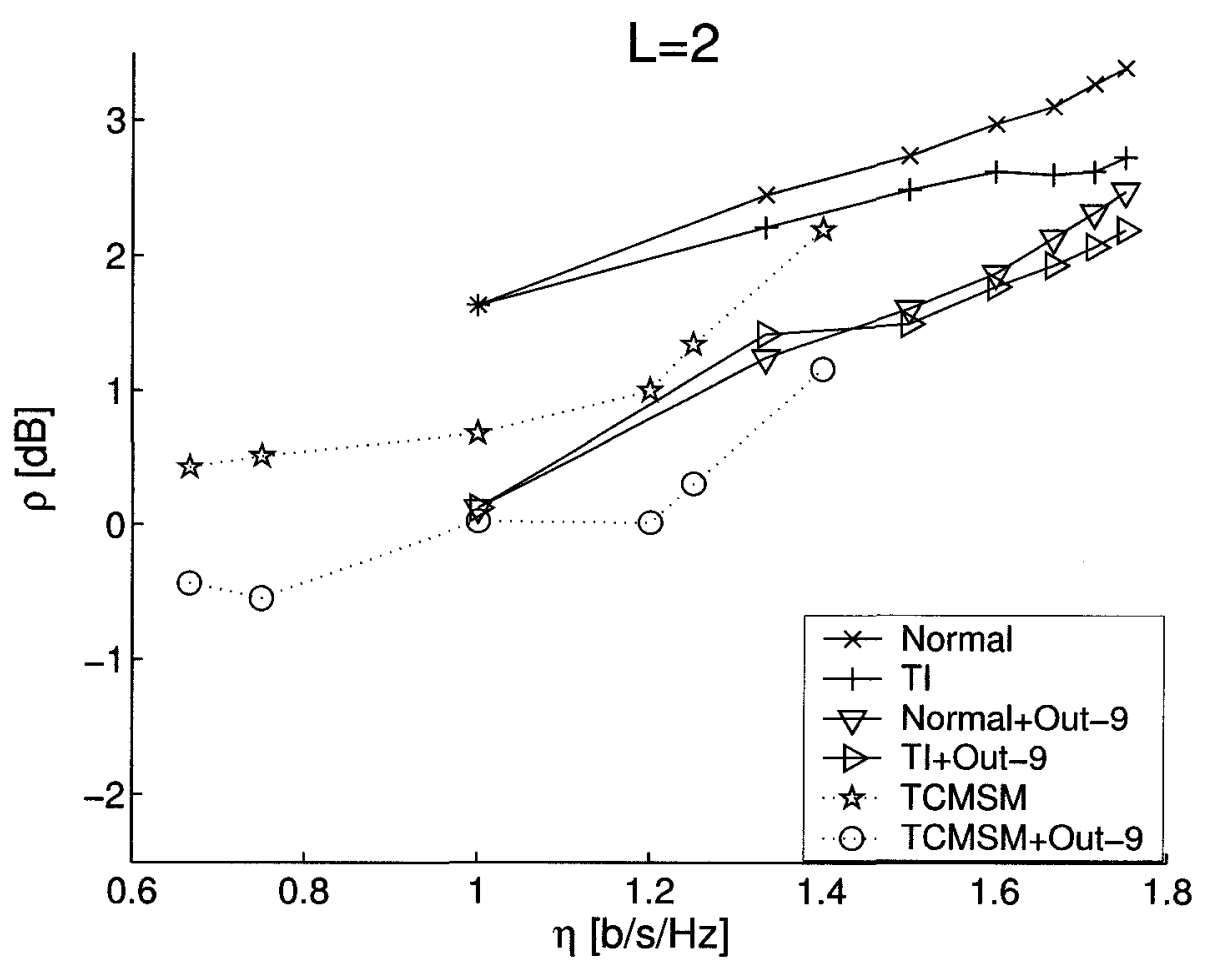

Figure A.5: Normalized average optical power $(\rho)$ versus bandwidth efficiency $(\eta)$ for scaled 9-APSK $L=2$ out-of-band techniques 


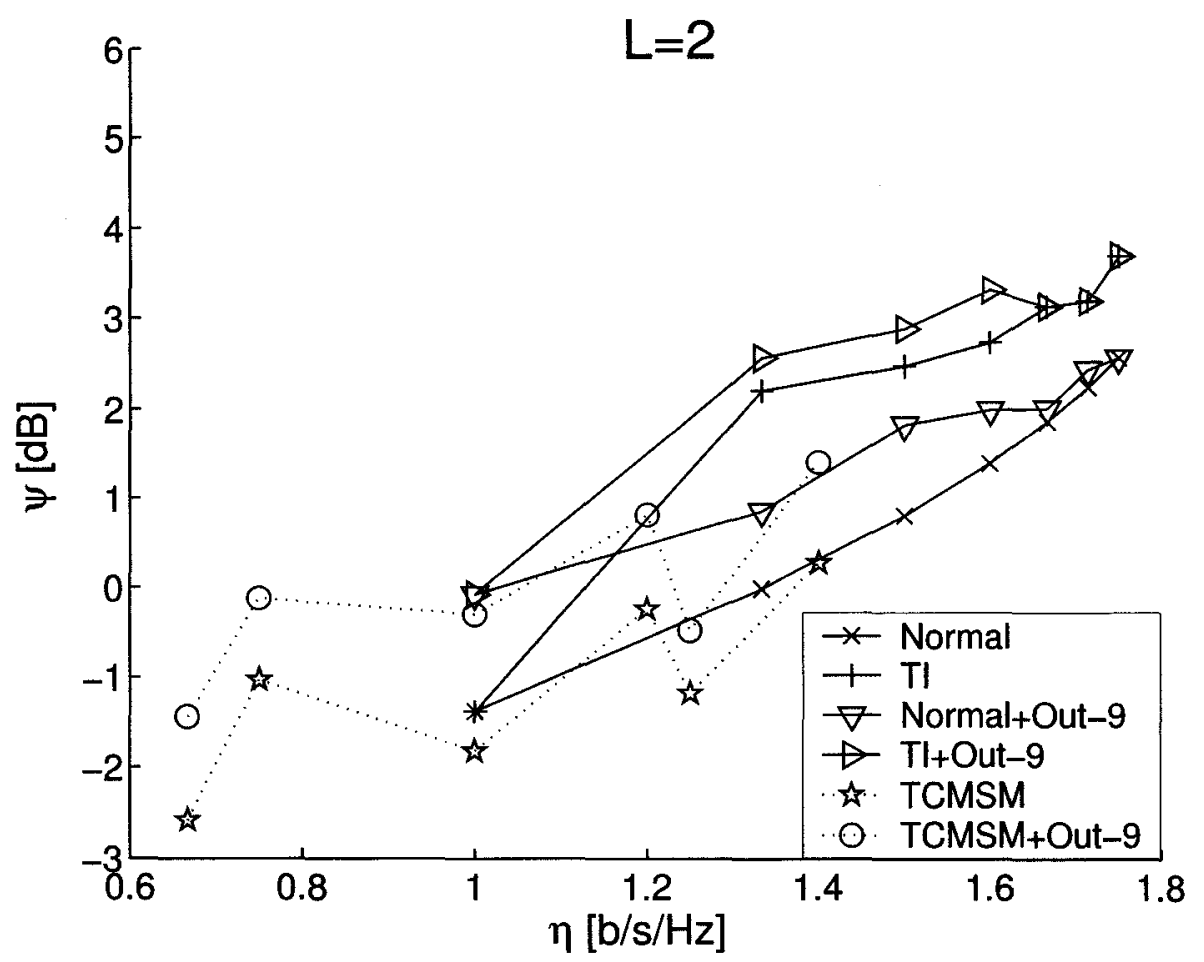

Figure A.6: Normalized peak optical power $(\psi)$ versus bandwidth efficiency $(\eta)$ for 9-APSK scaled $L=2$ out-of-band techniques 


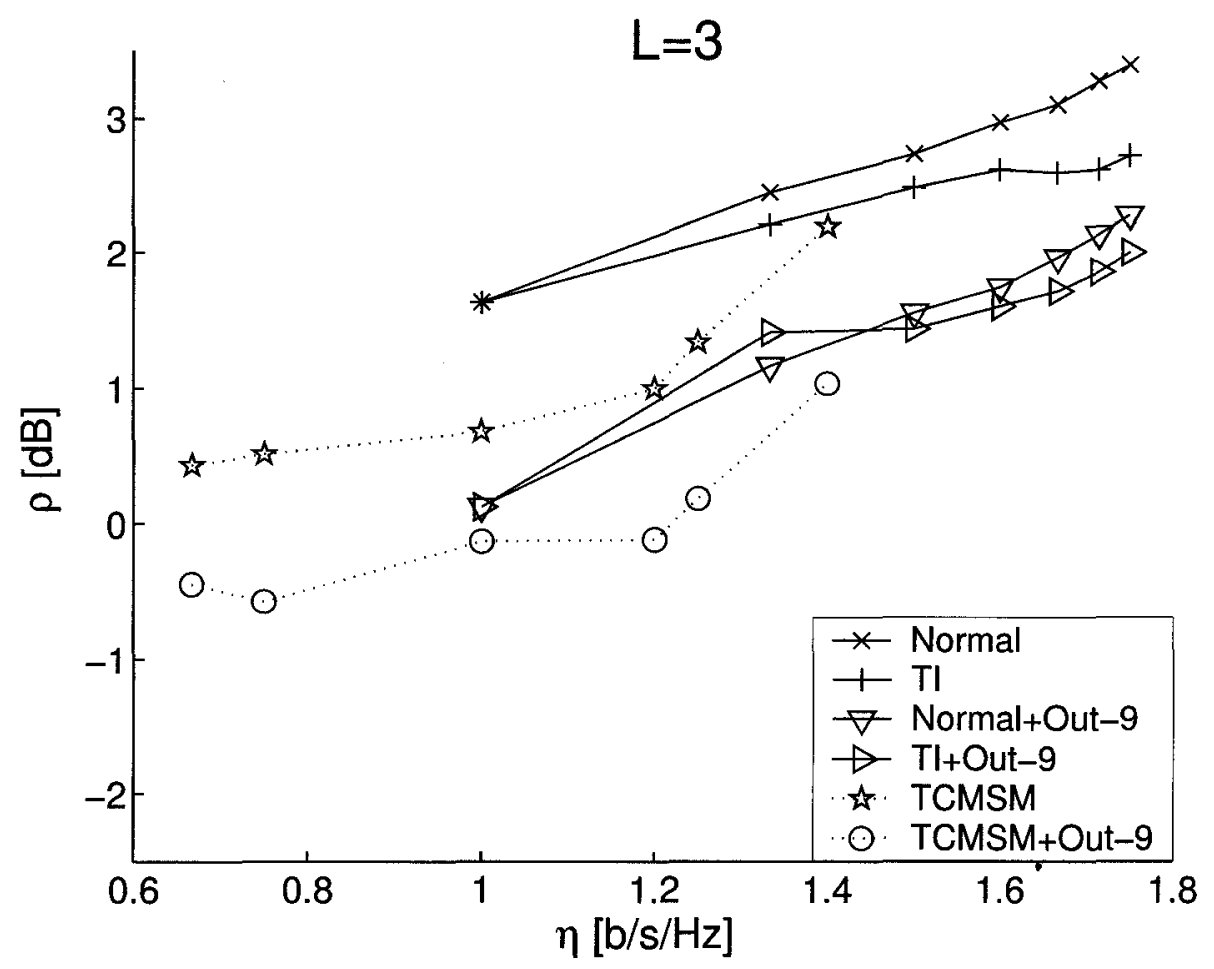

Figure A.7: Normalized average optical power $(\rho)$ versus bandwidth efficiency $(\eta)$ for scaled 9-APSK $L=3$ out-of-band techniques 


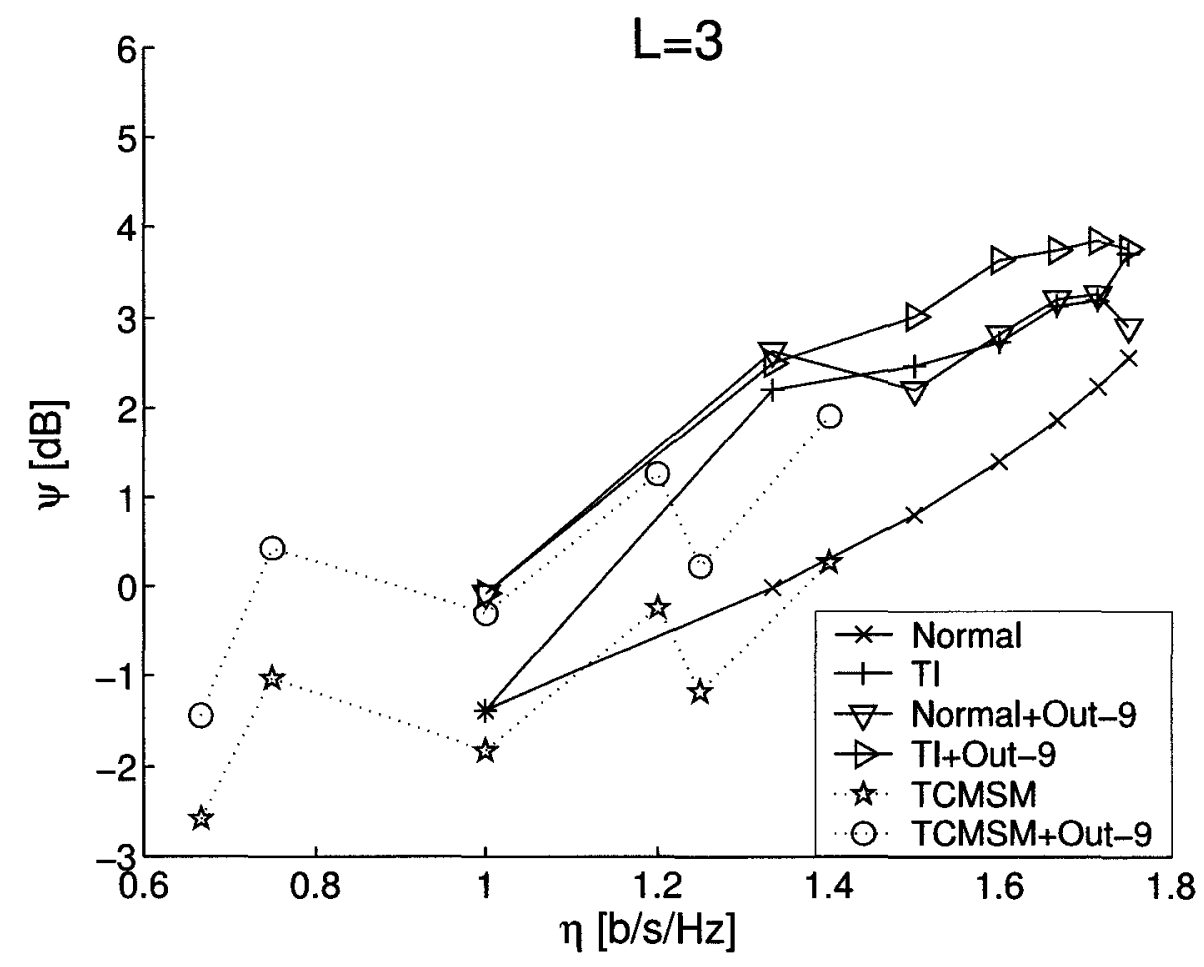

Figure A.8: Normalized peak optical power $(\psi)$ versus bandwidth efficiency $(\eta)$ for scaled 9-APSK $L=3$ out-of-band techniques 


\begin{tabular}{|c|c|c|c|c|}
\hline In-band & $\eta$ & Reduction of $\rho[\mathrm{dB}]$ & Increase in $\psi[\mathrm{dB}]$ & Memory [bytes] \\
\hline \multirow[t]{7}{*}{ Normal } & 1.00 & 2.09 & 2.37 & 64 \\
\hline & 1.33 & 1.99 & 1.46 & 256 \\
\hline & 1.50 & 1.67 & 1.06 & 1024 \\
\hline & 1.60 & 1.62 & 0.88 & 4096 \\
\hline & 1.67 & 1.45 & 0.68 & 16384 \\
\hline & 1.71 & 1.40 & 0.63 & 65536 \\
\hline & 1.75 & 1.35 & 0.59 & 262144 \\
\hline \multirow[t]{7}{*}{ TI } & 1.00 & 2.09 & 2.37 & 64 \\
\hline & 1.33 & 1.30 & 0.39 & 256 \\
\hline & 1.50 & 1.50 & 1.24 & 1024 \\
\hline & 1.60 & 1.28 & 0.88 & 4096 \\
\hline & 1.67 & 1.04 & 0.55 & 16384 \\
\hline & 1.71 & 0.87 & 1.16 & 65536 \\
\hline & 1.75 & 0.83 & 0.79 & 262144 \\
\hline \multirow[t]{6}{*}{ TCMSM } & 0.67 & 1.22 & 1.31 & 128 \\
\hline & 0.75 & 1.52 & 1.03 & 512 \\
\hline & 1.00 & 1.17 & 0.75 & 2048 \\
\hline & 1.20 & 1.53 & 0.89 & 4096 \\
\hline & 1.25 & 1.72 & 2.05 & 2048 \\
\hline & 1.40 & 1.57 & 1.55 & 8192 \\
\hline
\end{tabular}

Table A.1: Performance of $L=2$ real-amplitude out-of-band carriers applied to normal MSM systems and the best in-band trellis codes 


\begin{tabular}{|c|c|c|c|c|}
\hline In-band & $\eta$ & Reduction of $\rho[\mathrm{dB}]$ & Increase in $\psi[\mathrm{dB}]$ & Memory [bytes] \\
\hline \multirow[t]{7}{*}{ Normal } & 1.00 & 2.39 & 3.23 & 96 \\
\hline & 1.33 & 2.26 & 2.09 & 384 \\
\hline & 1.50 & 2.06 & 1.58 & 1536 \\
\hline & 1.60 & 1.87 & 1.24 & 6144 \\
\hline & 1.67 & 1.73 & 1.03 & 24576 \\
\hline & 1.71 & 1.67 & 1.01 & 98304 \\
\hline & 1.75 & 1.60 & 0.76 & 393216 \\
\hline \multirow[t]{7}{*}{$\mathrm{TI}$} & 1.00 & 2.39 & 3.23 & 96 \\
\hline & 1.33 & 1.50 & 0.41 & 384 \\
\hline & 1.50 & 1.81 & 1.78 & 1536 \\
\hline & 1.60 & 1.50 & 1.09 & 6144 \\
\hline & 1.67 & 1.33 & 1.26 & 24576 \\
\hline & 1.71 & 1.16 & 1.29 & 98304 \\
\hline & 1.75 & 1.10 & 1.09 & 393216 \\
\hline \multirow[t]{6}{*}{ TCMSM } & 0.67 & 1.33 & 2.22 & 192 \\
\hline & 0.75 & 1.86 & 1.39 & 768 \\
\hline & 1.00 & 1.51 & 1.08 & 3072 \\
\hline & 1.20 & 1.77 & 1.02 & 6144 \\
\hline & 1.25 & 1.82 & 1.50 & 3072 \\
\hline & 1.40 & 1.82 & 1.93 & 12288 \\
\hline
\end{tabular}

Table A.2: Performance of $L=3$ real-amplitude out-of-band carriers applied to normal MSM systems and the best in-band trellis codes 


\begin{tabular}{|c|c|c|c|c|}
\hline In-band & $\eta$ & Reduction of $\rho[\mathrm{dB}]$ & Increase in $\psi[\mathrm{dB}]$ & Memory [bytes] \\
\hline \multirow[t]{7}{*}{ Normal } & 1.00 & 1.50 & 1.30 & 12 \\
\hline & 1.33 & 1.21 & 0.87 & 36 \\
\hline & 1.50 & 1.13 & 1.02 & 132 \\
\hline & 1.60 & 1.10 & 0.59 & 516 \\
\hline & 1.67 & 0.97 & 0.15 & 2052 \\
\hline & 1.71 & 0.95 & 0.19 & 8196 \\
\hline & 1.75 & 0.91 & 0.00 & 32772 \\
\hline \multirow[t]{7}{*}{ TI } & 1.00 & 1.50 & 1.30 & 12 \\
\hline & 1.33 & 0.80 & 0.36 & 36 \\
\hline & 1.50 & 1.00 & 0.41 & 132 \\
\hline & 1.60 & 0.85 & 0.59 & 516 \\
\hline & 1.67 & 0.67 & 0.00 & 2052 \\
\hline & 1.71 & 0.56 & 0.00 & 8196 \\
\hline & 1.75 & 0.53 & 0.00 & 32772 \\
\hline \multirow[t]{6}{*}{ TCMSM } & 0.67 & 0.87 & 1.14 & 20 \\
\hline & 0.75 & 1.06 & 0.91 & 68 \\
\hline & 1.00 & 0.66 & 1.52 & 260 \\
\hline & 1.20 & 0.99 & 1.06 & 516 \\
\hline & 1.25 & 1.03 & 0.70 & 260 \\
\hline & 1.40 & 1.04 & 1.14 & 1028 \\
\hline
\end{tabular}

Table A.3: Performance of $L=2$ scaled 9-APSK out-of-band carriers applied to normal MSM systems and the best in-band trellis codes 


\begin{tabular}{|c|c|c|c|c|}
\hline In-band & $\eta$ & Reduction of $\rho[\mathrm{dB}]$ & Increase in $\psi[\mathrm{dB}]$ & Memory [bytes] \\
\hline \multirow[t]{7}{*}{ Normal } & 1.00 & 1.50 & 1.30 & 16 \\
\hline & 1.33 & 1.29 & 2.65 & 52 \\
\hline & 1.50 & 1.18 & 1.40 & 196 \\
\hline & 1.60 & 1.23 & 1.43 & 772 \\
\hline & 1.67 & 1.14 & 1.35 & 3076 \\
\hline & 1.71 & 1.13 & 1.02 & 12292 \\
\hline & 1.75 & 1.11 & 0.34 & 49156 \\
\hline \multirow[t]{7}{*}{$\mathrm{TI}$} & 1.00 & 1.50 & 1.30 & 16 \\
\hline & 1.33 & 0.80 & 0.30 & 52 \\
\hline & 1.50 & 1.05 & 0.55 & 196 \\
\hline & 1.60 & 1.02 & 0.90 & 772 \\
\hline & 1.67 & 0.89 & 0.60 & 3076 \\
\hline & 1.71 & 0.77 & 0.65 & 12292 \\
\hline & 1.75 & 0.73 & 0.06 & 49156 \\
\hline \multirow[t]{6}{*}{ TCMSM } & 0.67 & 0.87 & 1.14 & 28 \\
\hline & 0.75 & 1.08 & 1.46 & 100 \\
\hline & 1.00 & 0.81 & 1.52 & 388 \\
\hline & 1.20 & 1.11 & 1.50 & 772 \\
\hline & 1.25 & 1.15 & 1.41 & 388 \\
\hline & 1.40 & 1.16 & 1.64 & 1540 \\
\hline
\end{tabular}

Table A.4: Performance of $L=3$ scaled 9-APSK out-of-band carriers applied to normal MSM systems and the best in-band trellis codes 
efficiencies as did in Sections 4.2.2 and 4.3.2. On average, 1.65 dB of average optical power reduction is realized with a peak optical power increase of $1.10 \mathrm{~dB}$ by applying $L=2$ real-amplitude out-of-band carrier to a normal MSM system. By changing $L$ to 3 , the average optical power reduction is $1.94 \mathrm{~dB}$ on average, and the peak optical power increase is $1.56 \mathrm{~dB}$ on average.

In contrast, by applying $L=2$ real-amplitude out-of-band carriers to the best in-band trellis codes in Table 3.5, the average optical power reductions is $1.46 \mathrm{~dB}$ optical on average, and the increases in peak optical power is $1.26 \mathrm{~dB}$ optical on average. Using $L=3$ real-amplitude out-of-band carriers, the average optical power reductions is $1.69 \mathrm{~dB}$ optical on average, and the increases in peak optical power is $1.52 \mathrm{~dB}$ optical on average.

As is observed in Chapter 4, scaled 9-APSK out-of-band carriers achieve a smaller average optical power reduction compared to real-amplitude carriers. However, they offer a smaller increase in the peak optical power and a smaller memory requirement. Moreover, its transmitter is easier to implement since generating out-of-band carrier amplitudes over a regular constellation is easier than generating them over real numbers. Therefore, scaled 9-APSK out-of-band carriers require smaller additional complexity at the transmitter. In applications where the complexity is tightly limited and the size of the lookup table has to be even smaller, out-of-band carriers can be applied to a portion of the input symbols with high average optical power, as proposed in Section 4.4 . 


\section{Bibliography}

[1] F. R. Gfeller and U. Bapst, "Wireless in-house data communication via diffuse infrared radiation," Proc. IEEE, vol. 67, no. 11, pp. 1474-1486, Nov. 1979.

[2] J. M. Kahn and J. R. Barry, "Wireless infrared communications," Proc. IEEE, vol. 85, no. 2, pp. 265-298, Feb. 1997.

[3] S. Hranilovic, Wireless Optical Communication Systems. Springer, 2004.

[4] F. Parand, G. E. Faulkner, and D. C. O'Brien, "Cellular tracked optical wireless demonstration link," IEE Proc. Optoelectron., vol. 150, no. 5, pp. 490-496, Oct. 2003.

[5] S. T. Jivkova and M. Kavehrad, "Multispot diffusing configuration for wireless infrared access," IEEE Trans. Commun., vol. 48, no. 6, pp. 970-978, June 2000.

[6] J. M. Kahn, W. J. Krause, and J. B. Carruthers, "Experimental characterization of non-directed indoor infrared channels," IEEE Trans. Commun., vol. 43, no. 2/3/4, pp. 1613-1623, Feb./Mar./Apr. 1995.

[7] R.Narasimhan, M. D. Audeh, and J. M. Kahn, "Effect of electronic-ballast fluorescent lighting on wireless infrared links," IEE Proc. Optoelectron., vol. 143, no. 6 , pp. $347-354$, Dec. 1996. 
[8] G. Ungerboeck, "Channel coding with multilevel/phase signals," IEEE Trans. Inform. Theory, vol. IT-28, no. 1, pp. 55-67, Jan. 1982.

[9] USB Implementers Forum, "Web site," www.usb.org.

[10] IEEE, "Web site," www.ieee.org.

[11] T. S. Rappaport, Wireless Communications: Principles and Practice 2nd Edition. Prentice Hill, 2002.

[12] Bluetooth Special Interest Group, "Web site," www.bluetooth.org.

[13] Y.-W. Bai and C.-L. Chiang, "Design and implementation of the integration applications for a portable mp3 player with a bluetooth hand-free/set," IEEE Trans. Consumer Electron., vol. 51, no. 3, pp. 849-855, Aug. 2005.

[14] Bluetooth Special Interest Group, "Bluetooth specification 1.2," (available at www. bluetooth.org/spec), 2003.

[15] Infrared Data Association, "Web site," www.irda.org.

[16] D. R. Wisely, "A 1 Gbit/s optical wireless tracked architecture for ATM delivery," in Proc. IEE Colloquium on Optical Free Space Communication Links, London, U.K., Feb. 1996, pp. 14/1-14/7.

[17] J. B. Carruthers and J. M. Kahn, "Modeling of nondirected wireless infrared channels," IEEE Trans. Commun., vol. 45, no. 10, pp. 1260-1268, Oct. 1997.

[18] — - "Multiple-subcarrier modulation for nondirected wireless infrared communication," IEEE J. Select. Areas Commun., vol. 14, no. 3, pp. 538-546, Apr. 1996. 
[19] D. R. Wisely, "A $100 \mathrm{Mbit} / \mathrm{s}$ tracked optical wireless telepoint," in Proc. IEEE Int. Symp. Personal, Indoor and Mobile Radio Communications, vol. 3, Helsinki, Finland, Sept. 1997, pp. 964-968.

[20] V. Jungnickel, A. Forck, T. Haustein, U. Krüger, V. Pohl, and C. von Helmolt, "Electronic tracking for wireless infrared communications," IEEE Trans. Wireless Commun., vol. 2, no. 5, pp. 989-999, Sept. 2003.

[21] D. C. M. Lee, J. M. Kahn, and M. D. Audeh, "Trellis-coded pulse-position modulation for indoor wireless infrared communications," IEEE Trans. Commun., vol. 45, no. 9, pp. 1080-1086, Sept. 1997.

[22] D. C. Lee and J. M. Kahn, "Coding and equalization for PPM on wireless infrared channels," IEEE Trans. Commun., vol. 47, no. 2, pp. 255-260, Feb. 1999.

[23] H. Park and J. R. Barry, "Trellis-coded multiple-pulse-position modulation for wireless infrared communications," IEEE Trans. Commun., vol. 52, no. 4, pp. 643-651, Apr. 2004.

[24] G. W. Marsh and J. M. Kahn, "50-Mb/s diffuse infrared free-space link using on-off keying with decision-feedback equalization," IEEE Photon. Technol. Lett., vol. 6 , no. 10 , pp. $1268-1270$, Oct. 1994.

[25] — - "Performance evaluation of experimental $50-\mathrm{Mb} / \mathrm{s}$ diffuse infrared wireless link using on-off keying with decision-feedback equalization," IEEE Trans. Commun., vol. 44, no. 11, pp. 1496-1504, Nov. 1996.

[26] M. D. Audeh and J. M. Kahn, "Performance evaluation of baseband OOK for wireless indoor infrared LAN's operating at 100Mb/s," IEEE Trans. Commun., vol. 43, no. 6, pp. 2085-2093, June 1995. 
[27] D. C. Lee and J. M. Kahn, "Experimental 25-Mb/s wireless infrared link using 4-PPM with scalar decision-feedback equalization," in Proc. IEEE Int. Conf. on Commun., vol. 1, no. 7-11, Atlanta, Georgia, June 1998, pp. 26-30.

[28] D. C. O'Brien, G. E. Faulkner, E. B. Zyambo, K. Jim, D. J. Edwards, P. Stavrinou, G. Parry, J. Bellon, M. J. Sibley, V. A. Lalithambika, V. M. Joyner, R. J. Samsudin, D. M. Holburn, and R. J. Mears, "Integrated transceivers for optical wireless communications," IEEE J. Select. Topics Quantum Electron., vol. 11, no. 1, pp. 173-183, 2005.

[29] J. A. C. Bingham, "Multicarrier modulation for data transmission: An idea whose time has come," IEEE Commun. Mag., vol. 28, pp. 5-14, May 1990.

[30] T. E. Darcie, "Subcarrier multiplexing for lightwave networks and video distribution systems," IEEE J. Select. Areas Commun., vol. 8, no. 7, pp. 1240-1248, Sept. 1990.

[31] R. You and J. M. Kahn, "Average power reduction techniques for multiplesubcarrier intensity-modulated optical signals," IEEE Trans. Commun., vol. 49, no. 12, pp. 2164-2171, Dec. 2001.

[32] S. Teramoto and T. Ohtsuki, "Multiple-subcarrier optical communication systems with peak reduction carriers," in Proc. IEEE GLOBECOM, vol. 6, San Francisco Marriott, CA, Dec. 1-5 2003, pp. 3274-3278.

[33] N. Kitamoto and T. Ohtsuki, "Parallel combinatory multiple-subcarrier optical wireless communication systems," Int. J. Commun. Syst, vol. 18, pp. 195-203, Apr. 2005. 
[34] S. Teramoto and T. Ohtsuki, "Multiple-subcarrier optical communication systems with subcarrier signal-point sequence," IEEE Trans. Commun., vol. 53, no. 10 , pp. $1738-1743$, Oct. 2005.

[35] J. Tellado, Multicarrier Modulation with Low PAR: Applications to DSL and Wireless. Kluwer, 2000.

[36] K. G. Paterson and V. Tarokh, "On the existence and construction of good codes with low peak-to-average power ratios," IEEE Trans. Inform. Theory, vol. 46, no. 6, pp. 1974-1987, Sept. 2000.

[37] M. Sharif, M. Gharavi-Alkhansari, and B. H. Khalaj, "On the peak-to-average power of OFDM signals based on oversampling," IEEE Trans. Commun., vol. 51, no. 1, pp. 72-78, Jan. 2003.

[38] D. J. G. Mestdagh, P. Spruyt, and B. Biran, "Analysis of clipping effect in DMTbased ADSL systems," in Proc. IEEE SUPERCOMM/ICC, vol. 1, New Orleans, LA, May 1994, pp. 293-300.

[39] R. Gross and D. Veeneman, "SNR and spectral properties for a clipped DMT ADSL signal," in Proc. IEEE SUPERCOMM/ICC, vol. 2, New Orleans, LA, May 1994, pp. $843-847$.

[40] R. O'Neill and L. B. Lopes, "Performance of amplitude limited multitone signals," in Proc. IEEE 44th Veh. Technol. Conf., vol. 3, Stockholm, Sweden, June 1994, pp. $1675-1679$.

[41] —_ "Envelope variations and spectral splatter in clipped multicarrier signals," in Proc. IEEE Int. Symp. Personal, Indoor and Mobile Radio Communications, vol. 1, no. 27-29, Toronto, Canada, Sept. 1995, pp. $71-75$. 
[42] X. Li and J. Leonard J. Cimini, "Effects of clipping and filtering on the performance of OFDM," in Proc. IEEE 47th Veh. Technol. Conf., vol. 3, no. 4-7, Phoenix, AZ, May 1997, pp. $1634-1638$.

[43] R. van Nee and A. de Wild, "Reducing the peak-to-average power ratio of OFDM," in Proc. IEEE Conf. on Vehic. Technol., Ottawa, Canada, May 1998, pp. $2072-2076$.

[44] P. Pauli, M.; Kuchenbecker, "On the reduction of the out-of-band radiation of OFDM-signals," in Proc. IEEE Int. Conf. on Commun., vol. 3, no. 7-11, Atlanta, Georgia, June 1998, pp. $1304-1308$.

[45] J. S. Chow, J. A. C. Bingham, and M. S. Flowers, "Mitigating clipping noise in multi-carrier systems," in Proc. IEEE Int. Conf. on Commun., vol. 2, no. 8-12, Montreal, Canada, June 1997, pp. 715 - 719.

[46] D. Wulich, "Peak factor in orthogonal multicarrier modulation with variable levels," Electron. Lett., vol. 32, no. 205, 1996.

[47] B. M. Popovic, "Synthesis of power efficient multitone signals with flat amplitude spectrum," IEEE Trans. Commun., vol. 39, no. 7, pp. 1031 - 1033, 1991.

[48] K. G. Patterson, "Coding techniques for power controlled OFDM," in Proc. IEEE Int. Symp. Personal, Indoor and Mobile Radio Communications, vol. 2, no. 8-11, Boston, Mass., Sept. 1998, pp. $801-805$.

[49] J. A. Davis and J. Jedwab, "Peak-to-mean power control in OFDM, Golay complementary sequences, and Reed-Muller codes," IEEE Trans. Inform. Theory, vol. 45, no. 7, pp. 2397-2417, Nov. 1999. 
[50] C. V. Chong and V. Tarokh, "A simple encodable/decodable OFDM QPSK code with low peak-to-mean envelope power ratio," IEEE Trans. Inform. Theory, vol. 47, no. 7, pp. 3025-3029, Nov. 2001.

[51] D. Wulich, "Reduction of peak to mean ratio of multicarrier modulation using cyclic coding," Electron. Lett., vol. 32, no. 5, pp. 432-433, Feb. 1996.

[52] A. E. Jones, T. A. Wilkinson, and S. K. Barton, "Block coding scheme for reduction of peak to mean envelope power ratio of multicarrier transmission schemes," Electron. Lett., vol. 30, no. 25, pp. 2098-2099, Dec. 1994.

[53] T. A. Wilkinson and A. E. Jones, "Minimisation of the peak to mean envelope power ratio of multicarrier transmission schemes by block coding," in Proc. IEEE 45th Veh. Technol. Conf., vol. 2, no. 25-28, Chicago, IL, July 1995, pp. 825 829 .

[54] A. E. Jones and T. A. Wilkinson, "Combined coding for error control and increased robustness to system nonlinearities in OFDM," in Proc. IEEE 46th Veh. Technol. Conf., vol. 2, Atlanta, Georgia, Apr. 1996, pp. $904-908$.

[55] S. J. Shepherd, P. W. J. V. Eetvelt, C. W. W. Millington, and S. K. Barton, "Simple coding scheme to reduce peak factor in QPSK multicarrier modulation," Electron. Lett., vol. 31, no. 14, pp. 1131-1132, July 1995.

[56] D. J. G. Mestdagh and P. M. P. Spruyt, "A method to reduce the probability of clipping in DMT-based transceivers," IEEE Trans. Commun., vol. 44, no. 10, pp. 1234-1238, Oct. 1996.

[57] P. V. Eetvelt, G. Wade, and M. Tomlinson, "Peak to average power reduction for OFDM schemes by selective scrambling," Electron. Lett., vol. 32, no. 21, pp. 1963 - 1964, Oct. 1996. 
[58] S. H. Müller and J. B. Huber, "A comparison of peak power reduction schemes for OFDM," in Proc. GLOBECOM, vol. 1, no. 3-8, Phoenix, Nov. 1997, pp. 1 5.

[59] - , "A comparison of peak power reduction schemes for OFDM," in Proc. IEEE Int. Symp. Personal, Indoor and Mobile Radio Communications, vol. 3, no. 1-4, Helsinki, Finland, Sept. 1997, pp. 1090 - 1094.

[60] M. Friese, "Multicarrier modulation with low peak-to-average power ratio," Electron. Lett., vol. 32, no. 8, pp. 713 - 714, Apr. 1996.

[61] — - "Multicarrier modulation with low peak-to-average power ratio," in Proc. GLOBECOM, vol. 1, no. 3-8, Phoenix, Nov. 1997, pp. $290-294$.

[62] M. Sharif, C. Florens, M. Fazel, and B. Hassibi, "Amplitude and sign adjustment for peak-to-average-power reduction," IEEE Trans. Commun., vol. 53, no. 8, pp. 1243-1247, Aug. 2005.

[63] A. Gatherer and M. Polley, "Controlling clipping probability in DMT transmission," in Proc. 31st Asilomar Conf. on Signals, Systems and Computers, vol. 1, Pacific Grove, CA, Nov. 2-5 1998, pp. 578-584.

[64] J. Tellado and J. M. Cioffi, "Efficient algorithms for reducing PAR in multicarrier systems," in Proc. IEEE Intl. Symp on Info. Thy., New York, NY, Aug. 16-21 1998, p. 191.

[65] P. K. Frenger and N. A. B. Svensson, "Parallel combinatory OFDM signaling," IEEE Trans. Commun., vol. 47, no. 4, pp. 558-567, Apr. 1999.

[66] J. Tellado and J. M. Cioffi, "PAR reduction with minimal or zero bandwidth loss and low complexity," ANSI document,T1E1.4 Technical Subcommittee,98-173:112, June 1998. 
[67] S. Hranilovic and D. A. Johns, "A multilevel modulation scheme for high-speed wireless infrared communications," in Proc. IEEE Int. Symp. Circuits and Systems, Orlando, Florida, May30-June2 1999.

[68] J. G. Proakis, Digital Communications 4th Edition. McGraw-Hill, 2000.

[69] S. Haykin, Communication Systems 4th Edition. Wiley, 2001.

[70] International Electrotechnical Commission, "Web site," www.iec.ch.

[71] A. J. Viterbi, "Error bounds for convolutional codes and an asymtotically optimal decoding algorithm," IEEE Trans. Inform. Theory, vol. 13, no. 2, pp. 260-269, Apr. 1967.

[72] S. Hranilovic and F. R. Kschischang, "Optical intensity-modulated direct detection channels: signal space and lattice codes," IEEE Trans. Inform. Theory, vol. 49, no. 6 , pp. 1385-1399, June 2003.

[73] S. B. Weinstein and P. M. Ebert, "Data transmission by frequency-division multiplexing using the discrete Fourier transform," IEEE Trans. Commun., vol. 19, no. 5, pp. 628-634, Oct. 1971.

[74] H. Rohling, T. May, K. Brüninghaus, and R. Grünheid, "Broad-band OFDM radio transmission for multimedia applications," Proc. IEEE, vol. 87, no. 10, pp. 1778-1789, Oct. 1999.

[75] A. Peled and A. Ruiz, "Frequency domain data transmission using reduced computational complexity algorithms," in Proc. IEEE ICASSP, vol. 5, Denver, Colorado, Apr. 1980, pp. 964-967.

[76] S. Boyd and L. Vandenberghe, Convex Optimization. Cambridge University Press, 2004. 
[77] J. F. Sturm, "Using SeDuMi 1.02, a Matlab toolbox for optimization over symmetric cones," Optimization Methods and Software, vol. 11-12, no. 625-653., 1999, see http://sedumi.mcmaster.ca for updates.

[78] IEEE, "Standard for binary floating point arithmetic," ANSI/IEEE Standard 754-1985, 1985. 